In cooperation with the U.S. Environmental Protection Agency

\title{
Water-Quality Data for Selected Streams in the Mississippi Alluvial Plain Ecoregion, Northwestern Mississippi, Septem- ber - October 2007
}

Data Series 493 
Cover photographs. Left: Vaney Creek, Tallahatchie County, Mississippi; Right: Lampsilis sp. at Black Bayou, Sunflower County, Mississippi. 


\section{Water-Quality Data for Selected Streams in the Mississippi Alluvial Plain Ecoregion, Northwestern Mississippi, September- October 2007}

By Matthew B. Hicks and Shane J. Stocks

Prepared in cooperation with the U.S. Environmental Protection Agency

Data Series 493 


\title{
U.S. Department of the Interior \\ KEN SALAZAR, Secretary \\ U.S. Geological Survey \\ Marcia K. McNutt, Director
}

\section{U.S. Geological Survey, Reston, Virginia: 2010}

\author{
For more information on the USGS — the Federal source for science about the Earth, its natural and living resources, \\ natural hazards, and the environment, visit http://www.usgs.gov or call 1-888-ASK-USGS \\ For an overview of USGS information products, including maps, imagery, and publications, \\ visit http://www.usgs.gov/pubprod \\ To order this and other USGS information products, visit http://store.usgs.gov
}

\begin{abstract}
Any use of trade, product, or firm names is for descriptive purposes only and does not imply endorsement by the U.S. Government.

Although this report is in the public domain, permission must be secured from the individual copyright owners to reproduce any copyrighted materials contained within this report.
\end{abstract}

Suggested citation:

Hicks, M.B., and Stocks, S.J., 2010, Water-quality data for selected streams in the Mississippi Alluvial Plain ecoregion, northwestern Mississippi, September - October 2007: U.S. Geological Survey Data Series Report 493, 118 p. 


\section{Contents}

Abstract
Introduction.
Description of the Study Area
Purpose and Scope
Collection of Field Measurements
Procestion of Water Samples
Refults

\section{Figure}

1. Location of study area and water-quality sampling sites within the Mississippi Aluvial Plain ecoregion, northwestern Mississippi...

\section{Tables}

1. Station names, station numbers, and locations of stream sites sampled in the Mississippi Alluvial Plain ecoregion, northwestern Mississippi, SeptemberOctober 2007

2. Physical and chemical properties measured in the Mississippi Alluvial Plain ecoregion, northwestern Mississippi, September-October 2007

3. Summary of surface-water quality analytical results for stream sites sampled in the Mississippi Alluvial Plain ecoregion, northwestern Mississippi, September-October 2007

4. Physical property data recorded at half-hour intervals for station 07279836 Ark Bayou near Tibbs, Mississippi, September 17-19, 2007

5. Physical property data recorded at half-hour intervals for station 07279937 David Bayou near Sledge, Mississippi, September 17-19, 2007

6. Physical property data recorded at half-hour intervals for station 07279970 Bobo Bayou at Bobo, Mississippi, October 4-9, 2007

7. Physical property data recorded at half-hour intervals for station 07280555 Tillatoba Creek west of Charleston, Mississippi, September 24-27, 2007 
8. Physical property data recorded at half-hour intervals for station 07286006 Ascalmore Creek near Macel, Mississippi, October 3-9, $2007 .$.

9. Physical property data recorded at half-hour intervals for station 07286011 Brushy Creek near Macel, Mississippi, October 1-3, 2007.

10. Physical property data recorded at half-hour intervals for station 07287310 Spring Branch near Tchula, Mississippi, September 11-13, 2007 .36

11. Physical property data recorded at half-hour intervals for station 07288185 Black Bayou at Baltzer, Mississippi, October 4-9, 2007. .39

12. Physical property data recorded at half-hour intervals for station 07288280 Big Sunflower River near Merigold, Mississippi, September 24-27, 2007.

13. Physical property data recorded at half-hour intervals for station 07288365 Lead Bayou near Boyle, Mississippi, October 1-3, 2007.

14. Physical property data recorded at half-hour intervals for station 07288600 Quiver River near Moorhead, Mississippi, October 1-3, 2007 . .52

15. Physical property data recorded at half-hour intervals for station 07288656 Bogue Phalia near Darlove, Mississippi, October 2-4, 2007 .55

16. Physical property data recorded at half-hour intervals for station 07288792 Deer Creek northeast of Valley Park, Mississippi, September 10-14, 2007 .58

17. Physical property data recorded at half-hour intervals for station 325901090465500 Jaynes Bayou at Mouth near Anguilla, Mississippi, September 10-13, 2007 .63

18. Physical property data recorded at half-hour intervals for station 330730090431300 Murphy Bayou at Murphy, Mississippi, September 10-13, 2007

19. Physical property data recorded at half-hour intervals for station 332749090103400 Pelucia Creek near Rising Sun, Mississippi, October 1-3, 2007. 71

20. Physical property data recorded at half-hour intervals for station 333328090554800 Clear Creek near Stringtown, Mississippi, September 10-13, 2007. . .74

21. Physical property data recorded at half-hour intervals for station 333615090061800 Teoc Creek near Teoc, Mississippi, October 1-3, 2007 . .78

22. Physical property data recorded at half-hour intervals for station 334747090285000 Wild Bill Bayou near Drew, Mississippi, September 11-13, 2007

23. Physical property data recorded at half-hour intervals for station 335105090415300 Mound Bayou near Merigold, Mississippi, September 24-27, 2007

24. Physical property data recorded at half-hour intervals for station 335458090287000 Bear Bayou near Parchman, Mississippi, September 24-27, 2007

25. Physical property data recorded at half-hour intervals for station 335749090273300 Home Cypress Bayou near Rome, Mississippi, September 17-20, 2007

26. Physical property data recorded at half-hour intervals for station 335919090133600 Opossum Bayou near Webb, Mississippi, September 24-27, 2007

27. Physical property data recorded at half-hour intervals for station 340157090071200 Unnamed Tributary Panola Floodway near Charleston, Mississippi, September 24-27, 2007

28. Physical property data recorded at half-hour intervals for station 340423090210800 Hopson Bayou at Vance, Mississippi, October 4-9, 2007

29. Physical property data recorded at half-hour intervals for station 341640090055100 Unnamed Tributary to Tallahatchie River near Locke Station, Mississippi, September 17-19, 2007

30. Physical property data recorded at half-hour intervals for station 341701090113300 Ash Log Bayou near Bobo, Mississippi, September 17-19, 2007

31. Physical property data recorded at half-hour intervals for station 344516090164400 Floyd Bayou, near Green River, Mississippi, September 17-19, 2007. 


\section{Conversion Factors}

Inch/Pound to SI

\begin{tabular}{lcl}
\hline Multiply & By & To obtain \\
\hline & & Length \\
\hline $\begin{array}{l}\text { millimeter }(\mathrm{mm}) \\
\text { mile }(\mathrm{mi})\end{array}$ & 0.039 & inch (in.) \\
& 1.609 & kilometer $(\mathrm{km})$ \\
\hline square mile $\left(\mathrm{mi}^{2}\right)$ & Area & \\
\hline & 2.590 & square kilometer $\left(\mathrm{km}^{2}\right)$ \\
\hline gallon (gal) & Volume & \\
liter $(\mathrm{L})$ & 3.785 & liter $(\mathrm{L})$ \\
\hline & 0.26417 & gallon $(\mathrm{gal})$ \\
\hline cubic foot per second $\left(\mathrm{ft}^{3} / \mathrm{s}\right)$ & Flow & \\
\hline
\end{tabular}

SI to Inch/Pound

\begin{tabular}{lll}
\hline Multiply & By & To obtain \\
\hline \multicolumn{3}{c}{ Length } \\
\hline millimeter $(\mathrm{mm})$ & 0.03937 & inch (in.) \\
\hline \multicolumn{3}{c}{ Volume } \\
\hline liter $(\mathrm{L})$ & 0.2642 & gallon (gal) \\
\hline
\end{tabular}

Temperature in degrees Celsius $\left({ }^{\circ} \mathrm{C}\right)$ may be converted to degrees Fahrenheit $\left({ }^{\circ} \mathrm{F}\right)$ as follows:

${ }^{\circ} \mathrm{F}=\left(1.8 x^{\circ} \mathrm{C}\right)+32$

Specific conductance is given in microsiemens per centimeter at 25 degrees Celsius $(\mu \mathrm{S} / \mathrm{cm}$ at $\left.25^{\circ} \mathrm{C}\right)$.

Concentrations of chemical constituents in water are given either in milligrams per liter (mg/L) or micrograms per liter $(\mu \mathrm{g} / \mathrm{L})$. 


\title{
Water-Quality Data for Selected Streams in the Mississippi Alluvial Plain Ecoregion, Northwestern Mississippi, September-October 2007
}

\author{
By Matthew B. Hicks and Shane J. Stocks
}

\section{Abstract}

From September through October 2007, the U.S. Geological Survey, in cooperation with the U.S. Environmental Protection Agency, collected and analyzed water-quality samples from streams in the Yazoo River basin within the Mississippi Alluvial Plain ecoregion in northwestern Mississippi. Water-quality samples were collected at 56 sites in the study area and analyzed for various physical and chemical characteristics including, but not limited to, suspended sediment, nutrients, and chlorophyll $a$. Additionally, water temperature, $\mathrm{pH}$, specific conductance, and dissolved oxygen data were measured at 28 of the sites using multiparameter water-quality meters at 30-minute intervals for a minimum of 48 hours. Data collected for this project will be used in the development of water-quality criteria for nutrients. The nutrient data will enhance existing datasets and support evaluation of cause and effect relations for nutrient criteria development. In addition, these indicators will assist in the development and evaluation of restoration and remediation plans for water bodies not meeting their designated uses, as stated in the U.S. Environmental Protection Agency's Clean Water Act Section 303(d).

\section{Introduction}

The U.S. Environmental Protection Agency (USEPA), under the authority of the Clean Water Act, requires States to identify impaired waters and develop remediation and restoration measures to improve water quality. The identification of impaired waters and understanding the causes and sources of impairment can be achieved by using scientifically based water-quality standards and criteria for physical, chemical, and biological attributes of surface waters. However, criteria of many physical, chemical, and biological properties have not been developed for streams in the Mississippi Aluvial Plain (MAP) ecoregion in northwestern Mississippi, all of which are part of the Yazoo River basin. The lack of criteria for this region makes it difficult to identify impaired waters, determine cause of impairment, and understand achievable target levels for remediation efforts. Since 2000, the USEPA has published more than 26 ecoregional nutrient criteria, which underscore a national focus on developing ecoregional nutrient-specific water-quality criteria for surface waters. The urgency and importance of developing nutrient standards and criteria for streams in the MAP ecoregion in northwestern Mississippi have been further magnified by an interest in understanding magnitudes and sources of nutrient loadings to the Gulf of Mexico.

The proximity of the Yazoo River basin to the Gulf of Mexico, the intensive agricultural land use within the basin, and the relatively small amount of data have lead to the speculation that the Yazoo River basin may be contributing a disproportionate amount of nutrients into the Mississippi River and hence to the Gulf of Mexico (Kleiss and others, 2000). Attempts have been made to better understand and characterize the nutrient loadings from the Yazoo River basin to the Mississippi River through data collection, data analyses, and modeling (Alexander and others, 1997; Runner and others, 2002). In 2002, a collaborative effort was initiated among many State and Federal agencies to collect data in the MAP portion of the Yazoo River basin, including nutrient concentrations and fish and macroinvertebrate biological response indicators (Rebich and others, 2004). Since 2002, data collection has continued to further the development of a robust water-quality and biological database for this region (Bryson and others, 2007).

\section{Description of the Study Area}

The Yazoo River basin in northwestern Mississippi drains to the Mississippi River and ultimately to the Gulf of Mexico and is the largest river basin in Mississippi encompassing about 13,400 square miles $\left(\mathrm{mi}^{2}\right)$. The Yazoo River basin is characterized by an area of uplands and an area of lowlands, each of which makes up approximately half of the entire basin area. The lowland area is locally referred to as the Delta, and lies within the MAP ecoregion. A steeply sloped land form creates the Bluff Hills that separate the lowland and upland regions of the Yazoo River basin. The Yazoo River is formed by the confluence of the Yalobusha and Tallahatchie Rivers, both of which have headwaters in the uplands. The Yazoo River flows southward until it reaches the Mississippi River at Vicksburg, MS. Two large river systems, the Sunflower River and Steele Bayou, merge with the Yazoo River above 
its confluence with the Mississippi River. The headwaters of these two river systems originate in the lowlands area of the basin, and both rivers remain in the lowlands until their confluence with the Yazoo River (fig. 1). The Yazoo River basin is sparsely populated, and the majority of the land use in the basin is agricultural (U.S. Geological Survey, 1990).

\section{Purpose and Scope}

The purpose of this report is to present data collected, including but not limited to nitrogen species, phosphorus species, chlorophyll $a$, suspended sediment, and oxygen to provide a stronger database for developing scientifically defensible nutrient criteria. The U.S. Geological Survey (USGS), in cooperation with the USEPA, collected waterquality data from September through October 2007 at 56 sites located along streams in the MAP ecoregion in northwestern Mississippi. Data from this study will be added to the existing database of chemical, physical, and biological data to strengthen analysis and evaluation of cause and effect relations in determination of nutrient criteria for rivers and streams in the MAP ecoregion in northwestern Mississippi and to assist in the development and evaluation of restoration and remediation plans for water bodies not meeting their designated uses. This report describes the data-collection and analytical methods used in this study and presents, in tabular form, the data collected.

\section{Methods}

Water-quality data collection consisted of measurements of physical and chemical properties in the field and water samples for laboratory analysis at each of the 56 sites. In addition, continuous measurements were recorded for a minimum of 48 hours at 28 of the 56 sites, using deployed multi-parameter water-quality sondes. The USGS and Mississippi Department of Environmental Quality (MDEQ) station numbers, station names, and location information for the sampling sites are listed in table 1. Physical and chemical properties measured in the field or analyzed in the laboratory are listed in table 2. Field-data collection was conducted by the USGS. Field methods used in collecting water samples for chemical analysis followed the USGS National Field Manual for the Collection of Water-Quality Data (Wilde and Radtke, 1998). Sample processing and laboratory analyses followed guidelines in the MDEQ Quality Assurance Project Plan (Mississippi Department of Environmental Quality, 2007) and the 2006 Quality Assurance Plan for Water-Quality Activities in the USGS Mississippi Water Science Center (R.A. Rebich, U.S. Geological Survey, written commun., 2006).

\section{Collection of Field Measurements}

Field measurements of five water-quality characteristics (water temperature, specific conductance, $\mathrm{pH}$, dissolvedoxygen concentration, and turbidity) were made by using YSI 6920 V2-1 Multi-Parameter Water-Quality Sondes, YSI 600XLM Multi-Parameter Water-Quality Sondes, and a Hach 2100 Portable Turbidimeter. Field measurements were made at all 56 sites at the time of water-sample collection using the YSI 600XLM sonde and a Hach 2100 Portable Turbidimeter. YSI 6920 sondes were deployed and left for a minimum of 48 hours at 28 of the 56 sites, and continuous measurements were made at 30-minute intervals. Reference measurements were made for quality-control purposes, using the YSI 600XLM sonde that was placed directly beside the deployed sonde at the same depth at the time of deployment and retrieval of deployed. Reference and field measurements were made at mid-depth and in the middle of the channel thalweg. Sondes deployed for continuous 30-minute interval readings were placed in the water column facing upstream and secured to a metal fence post with plastic ties. Prior to deployment, $\mathrm{pH}$ was calibrated at 4, 7, and 10; specific conductance was calibrated with a 1-point calibration at either 180,250 , or 500 microsiemens per centimeter $(\mu \mathrm{S} / \mathrm{cm})$ at 25 degrees Celsius and verified with a known standard on the high and low end of the calibrated value; dissolved oxygen was calibrated to 100-percent saturation; and turbidity was calibrated to 0 (deionized water), 40, and 100 nephelometric turbidity units (NTU). All pre-calibration readings were recorded. Immediately after the sonde was retrieved from deployment onsite, both the reference and deployed sondes were placed in a 5-gallon plastic bucket filled with streamwater, and physical measurements were recorded. The deployed sonde was cleaned and rinsed and placed back into the bucket with the reference sonde, and physical measurements were recorded a second time. Finally, deployed sondes were calibrated in the laboratory after returning from the field, and post-calibration readings were recorded. These data were used to quantify change over time in recorded values measured during deployment, which could have been attributed to fouling, drift, or equipment failure. The maximum change measured for the deployment period was $\leq \pm 0.2$ standard $\mathrm{pH}$ units, $\leq \pm 10$ percent for specific conductance, $\leq$ $\pm 0.5 \mathrm{mg} / \mathrm{L}$ of dissolved oxygen, and $\leq \pm 10$ percent for turbidity, all considered good or excellent based on data quality objectives (Mississippi Department of Environmental Quality, 2007). Therefore, readings for $\mathrm{pH}$, specific conductance, and dissolved oxygen were not corrected for drift. However, some values for turbidity were measured below 0.0 (negative values measured). For sites where turbidity values were below 0.0 , all turbidity data for that site were omitted from this report. Streamflow measurements were measured or estimated at most sites, and if flow was not present, no streamflow data were collected. Although some sites did not have flow, field measurements of physical properties were made, and water samples were collected. 


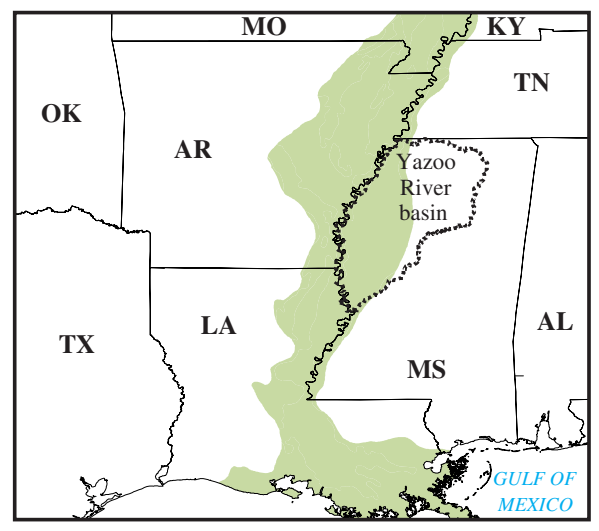

\section{EXPLANATION}

Mississippi Alluvial

Plain ecoregion V3 Water-quality sampling
site and number

$\nabla 2 \quad$ Water-quality and sonde sampling site and number

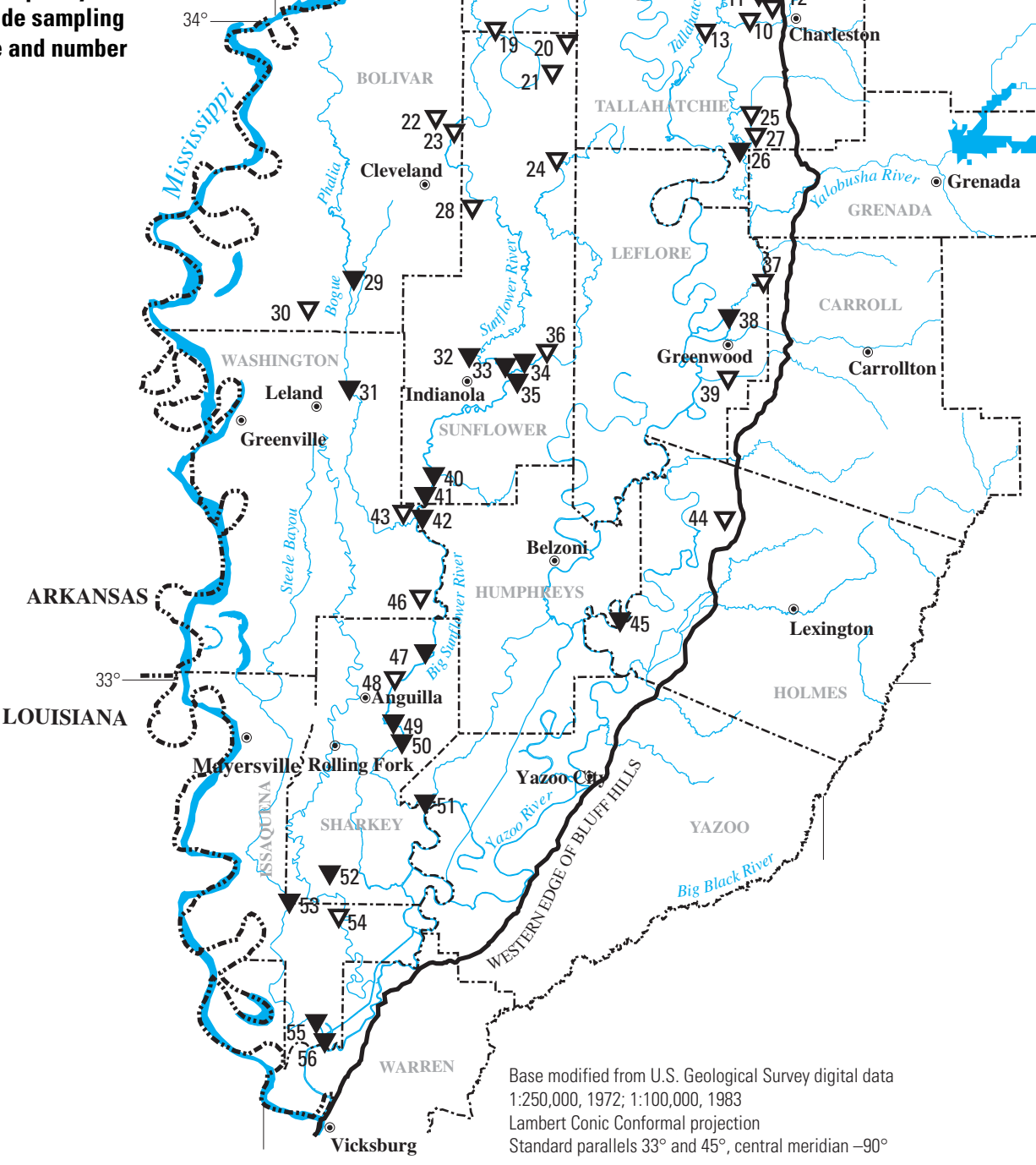

Figure 1. Location of study area and water-quality sampling sites in the Mississippi Aluvial Plain ecoregion, northwestern Mississippi. 


\section{Collection of Water Samples}

Water samples were collected either by wading into the stream, from a bridge by using a cabled sampler, or from a boat in remote areas. At stream sites with flow, a minimum of five 1-liter (L) bottles were filled from equal width/discharge transects across the stream and depth integrated. All 1-L bottle samples were used for onsite processing.

At sites with no flow, grab samples were collected by placing sample containers directly into the stream at midchannel and mid-depth. All sampling equipment was constructed of either Teflon ${ }^{\circledR}$, glass, or stainless steel, and was cleaned prior to sample collection with 0.2-percent solution of nonphosphate detergent, rinsed with tap water and deionized water, allowed to dry in a dust-free area, and wrapped in aluminum foil or placed in plastic bags for protection.

\section{Processing of Water Samples}

The 1-L glass bottles collected at sites with streamflow were composited into a churn that was pre-rinsed onsite. All onsite filtering was performed using a low vacuum pump. All orthophosphate samples were filtered using a capsule filter containing a 0.45 -micron filter and a 100 -milliliter $(\mathrm{mL})$ plastic filtration cup to capture the filtered water. The $100-\mathrm{mL}$ plastic filtration cup was immediately placed on ice and remained chilled until delivered to the laboratory. Chlorophyll $a$ samples were processed by filtering $25 \mathrm{~mL}$ of water through a 0.47 -millimeter $(\mathrm{mm})$ baked glass-fiber filter three times, for a total of three filters per site. Each filter was folded in half and enclosed inside a two-piece petri dish, and all three petri dishes were wrapped together in aluminum foil and packed on dry ice. All water samples were delivered for analysis to the MDEQ laboratory in Pearl, MS, within 48 hours of collection, with the exception of samples for suspended sediment analysis, which were delivered to the USGS sediment laboratory in Baton Rogue, LA.

Quality-control procedures were followed according to the MDEQ Quality Assurance Project Plan (Mississippi Department of Environmental Quality, 2007) and the 2006 Quality Assurance Plan for Water-Quality Activities in the USGS Mississippi Water Science Center (R.A. Rebich, U.S. Geological Survey, written commun., 2006). Split replicate samples were collected at 20 percent of the sites and sent to the USGS National Water Quality Laboratory in Lakewood, $\mathrm{CO}$, for quality assurance. Field replicate samples were collected at 10 percent of the 56 sites and sent to the MDEQ laboratory in Pearl, MS, for field-collection quality assurance.

\section{Results}

Water-quality data for 56 sites sampled in the MAP ecoregion, northwestern Mississippi, during SeptemberOctober 2007 are listed in table 3. Physical properties recorded continuously at 30 -minute intervals at 28 of the 56 project sites during September-October 2007 are listed in tables 4-31. Data results are stored electronically at the USGS Mississippi Water Science Center, in Jackson, MS, and can be accessed at http://waterdata.usgs.gov/ms/nwis.

\section{References Cited}

Alexander, R.B., Smith, R.A., and Schwarz, G.E., 1997, The regional transport of point and nonpoint-source nitrogen to the Gulf of Mexico, Proceedings of the First Gulf of Mexico Hypoxia Management Conference, December 5-6, 1995, Kenner, Louisiana: U.S. Environmental Protection Agency, EPA-55-R-97-001, p. 127-132.

Bryson, J.R., Coupe, R.H., and Manning, M.A., 2007, Characterization of water quality in unmonitored streams in the Mississippi alluvial plain, northwestern Mississippi, MayJune 2006: U.S. Geological Survey Scientific Investigations Report 2007-5116, 20 p.

Kleiss, B.A., Coupe, R.H., Gonthier, G.J., and Justus, B.J., 2000, Water quality in the Mississippi Embayment: Mississippi, Louisiana, Arkansas, Missouri, Tennessee, and Kentucky, 1995-98: U.S. Geological Survey Circular 1208, $36 \mathrm{p}$.

Mississippi Department of Environmental Quality, 2007, Quality assurance project plan for the data collection and analyses to support nutrient criteria development and use attainability analyses in the Mississippi Delta, $33 \mathrm{p}$.

Rebich, R.A., Welch, H.L., and Coupe, R.H., 2004, Evaluation of water-quality and habitat assessment data to determine ranges in stream conditions in the Mississippi River Alluvial Plain of northwestern Mississippi and eastern Arkansas: U.S. Geological Survey Water-Resources Investigations Report 03-4251, 47 p.

Runner, M.S., Turnipseed, D.P., and Coupe, R.H., 2002, Streamflow and nutrient data for the Yazoo River below Steele Bayou near Long Lake, Mississippi, 1996-2000: U.S. Geological Survey Water-Resources Investigations Report 02-4215, 35 p.

U.S. Geological Survey, 1990, USGS GeoData 1:250,000 and 1:100,000 Scale Land Use and Land Cover (LULC) and associated maps, digital data: U.S. Geological Survey geographic data download, accessed November 12, 2009, at http://edc2.usgs.gov/geodata/index.php

Wilde, F.D., and Radtke, D.B., 1998, National field manual for the collection of water-quality data: U.S. Geological Survey Techniques of Water-Resources Investigations, book 9 [variously paged]. 
Table 1. Station names, station numbers, and locations of stream sites sampled in the Mississippi Alluvial Plain ecoregion, northwestern Mississippi, September-0ctober 2007.

[MDEQ, Mississippi Department of Environmental Quality; USGS, U.S. Geological Survey; nr, near; MS, Mississippi; NE, northeast; Station numbers in bold were sampled for continuous physical properties at 30-minute intervals in addition to an instantaneous measurement for all other chemical characteristics]

\begin{tabular}{|c|c|c|c|c|}
\hline $\begin{array}{l}\text { USGS station } \\
\text { number }\end{array}$ & $\begin{array}{l}\text { MDEO station } \\
\text { number }\end{array}$ & USGS station name & $\begin{array}{l}\text { Latitude } \\
\text { (Degrees Min- } \\
\text { utes Seconds) }\end{array}$ & $\begin{array}{l}\text { Longitude (Degrees } \\
\text { Minutes Seconds) }\end{array}$ \\
\hline 07273610 & E117 & $\begin{array}{l}\text { Panola Quitman Floodway nr } \\
\text { Charleston, MS }\end{array}$ & $34^{\circ} 02^{\prime} 03^{\prime \prime}$ & $90^{\circ} 07^{\prime} 31^{\prime \prime}$ \\
\hline 07279140 & E108 & $\begin{array}{l}\text { Lake Cormorant Bayou nr Lake } \\
\text { Cormorant, MS }\end{array}$ & $34^{\circ} 51^{\prime} 37^{\prime \prime}$ & $90^{\circ} 12^{\prime} 52^{\prime \prime}$ \\
\hline 07279299 & E109 & Buck Island Bayou near Prichard, MS & $34^{\circ} 42^{\prime} 26^{\prime \prime}$ & $90^{\circ} 14^{\prime} 27^{\prime \prime}$ \\
\hline 07279748 & E110 & $\begin{array}{l}\text { White Oak Bayou at McDonald Road nr } \\
\text { Tibbs, MS }\end{array}$ & $34^{\circ} 30^{\prime} 59^{\prime \prime}$ & $90^{\circ} 19^{\prime} 17^{\prime \prime}$ \\
\hline 07279836 & D001 & Ark Bayou nr Tibbs, MS & $34^{\circ} 27^{\prime} 20^{\prime \prime}$ & $90^{\circ} 22^{\prime} 20^{\prime \prime}$ \\
\hline 07279937 & D002 & David Bayou nr Sledge, MS & $34^{\circ} 25^{\prime} 14^{\prime \prime}$ & $90^{\circ} 14^{\prime} 02^{\prime \prime}$ \\
\hline 07279970 & D031 & BoBo Bayou at Bobo, MS & $34^{\circ} 17^{\prime} 00^{\prime \prime}$ & $90^{\circ} 10^{\prime} 32^{\prime \prime}$ \\
\hline 07280555 & D023 & Tillatoba Creek west of Charleston, MS & $34^{\circ} 00^{\prime} 07^{\prime \prime}$ & $90^{\circ} 07^{\prime} 38^{\prime \prime}$ \\
\hline 07280810 & E106 & Cassidy Bayou nr Mattson, MS & $34^{\circ} 08^{\prime} 22^{\prime \prime}$ & $90^{\circ} 27^{\prime} 17^{\prime \prime}$ \\
\hline 07280851 & E107 & Cassidy Bayou East of Vance, MS & $34^{\circ} 05^{\prime} 02^{\prime \prime}$ & $90^{\circ} 19^{\prime} 15^{\prime \prime}$ \\
\hline 07286006 & D026 & Ascalmore Creek nr Macel, MS & $33^{\circ} 50^{\prime} 20^{\prime \prime}$ & $90^{\circ} 07^{\prime} 56^{\prime \prime}$ \\
\hline 07286011 & D003 & Brushy Creek nr Macel, MS & $33^{\circ} 48^{\prime} 52^{\prime \prime}$ & $90^{\circ} 07^{\prime} 34^{\prime \prime}$ \\
\hline 07286012 & D030 & Tippo Bayou nr Macel, MS & $33^{\circ} 48^{\prime} 33^{\prime \prime}$ & $90^{\circ} 08^{\prime} 34^{\prime \prime}$ \\
\hline 07286955 & E111 & $\begin{array}{l}\text { Big Sand Creek at Mouth nr } \\
\text { Greenwood, MS }\end{array}$ & $33^{\circ} 33^{\prime} 40^{\prime \prime}$ & $90^{\circ} 10^{\prime} 02^{\prime \prime}$ \\
\hline 07287309 & E112 & Tchula Lake nr Thorton, MS & $33^{\circ} 05^{\prime} 37^{\prime \prime}$ & $90^{\circ} 21^{\prime} 43^{\prime \prime}$ \\
\hline 07287310 & D022 & Spring Branch nr Tchula, MS & $33^{\circ} 14^{\prime} 31^{\prime \prime}$ & $90^{\circ} 11^{\prime} 02^{\prime \prime}$ \\
\hline 07288010 & E009 & Big Sunflower River at Hopson Spur, MS & $34^{\circ} 09^{\prime} 23^{\prime \prime}$ & $90^{\circ} 32^{\prime} 59^{\prime \prime}$ \\
\hline 07288140 & E113 & Howden Lake nr Duncan, MS & $34^{\circ} 03^{\prime} 14^{\prime \prime}$ & $90^{\circ} 41^{\prime} 46^{\prime \prime}$ \\
\hline 07288185 & D032 & Black Bayou at Baltzer, MS & $33^{\circ} 59^{\prime} 00^{\prime \prime}$ & $90^{\circ} 35^{\prime} 39^{\prime \prime}$ \\
\hline 07288280 & D012 & Big Sunflower River nr Merigold, MS & $33^{\circ} 49^{\prime} 57^{\prime \prime}$ & $90^{\circ} 40^{\prime} 12^{\prime \prime}$ \\
\hline 07288365 & D015 & Lead Bayou nr Boyle, MS & $33^{\circ} 43^{\prime} 07^{\prime \prime}$ & $90^{\circ} 38^{\prime} 34^{\prime \prime}$ \\
\hline 07288600 & D020 & Quiver River nr Moorhead, MS & $33^{\circ} 29^{\prime} 16^{\prime \prime}$ & $90^{\circ} 31^{\prime} 06^{\prime \prime}$ \\
\hline 07288607 & E114 & Porter Bayou nr Indianola, MS & $33^{\circ} 29^{\prime} 19^{\prime \prime}$ & $90^{\circ} 38^{\prime} 34^{\prime \prime}$ \\
\hline 07288609 & E124 & $\begin{array}{l}\text { Quiver River above the mouth nr } \\
\text { Moorehead, MS }\end{array}$ & $33^{\circ} 28^{\prime} 29^{\prime \prime}$ & $90^{\circ} 33^{\prime} 15^{\prime \prime}$ \\
\hline 07288610 & E012 & Big Sunflower River nr Moorehead, MS & $33^{\circ} 27^{\prime} 44^{\prime \prime}$ & $90^{\circ} 33^{\prime} 45^{\prime \prime}$ \\
\hline 07288624 & E014 & Big Sunflower River at Kinlock, MS & $33^{\circ} 18^{\prime} 34^{\prime \prime}$ & $90^{\circ} 42^{\prime} 23^{\prime \prime}$ \\
\hline 07288643 & E027 & Bogue Phalia nr Shaw, MS & $33^{\circ} 36^{\prime} 10^{\prime \prime}$ & $90^{\circ} 51^{\prime} 10^{\prime \prime}$ \\
\hline 07288647 & E127 & Bogue Phalia above Elizabeth, MS & $33^{\circ} 26^{\prime} 49^{\prime \prime}$ & $90^{\circ} 51^{\prime} 32^{\prime \prime}$ \\
\hline 07288656 & E115 & Bogue Phalia nr Darlove, MS & $33^{\circ} 15^{\prime} 15^{\prime \prime}$ & $90^{\circ} 43^{\prime} 54^{\prime \prime}$ \\
\hline 07288720 & E008 & Big Sunflower River at Holly Bluff, MS & $32^{\circ} 48^{\prime} 49^{\prime \prime}$ & $90^{\circ} 43^{\prime} 08^{\prime \prime}$ \\
\hline 07288792 & D010 & Deer Creek NE of Valley Park, MS & $32^{\circ} 38^{\prime} 05^{\prime \prime}$ & $90^{\circ} 51^{\prime} 47^{\prime \prime}$ \\
\hline 07288908 & E118 & Steele Bayou below Onward, MS & $32^{\circ} 40^{\prime} 11^{\prime \prime}$ & $90^{\circ} 57^{\prime} 17^{\prime \prime}$ \\
\hline
\end{tabular}


Table 1. Station names, station numbers, and locations of stream sites sampled in the Mississippi Alluvial Plain ecoregion, northwestern Mississippi, September-October 2007.-Continued

[MDEQ, Mississippi Department of Environmental Quality; USGS, U.S. Geological Survey; nr, near; MS, Mississippi; NE, northeast; Station numbers in bold were sampled for continuous physical properties at 30-minute intervals in addition to an instantaneous measurement for all other chemical characteristics]

\begin{tabular}{|c|c|c|c|c|}
\hline $\begin{array}{l}\text { USGS station } \\
\text { number }\end{array}$ & $\begin{array}{l}\text { MDEQ station } \\
\text { number }\end{array}$ & USGS station name & $\begin{array}{c}\text { Latitude } \\
\text { (Degrees Min- } \\
\text { utes Seconds) }\end{array}$ & $\begin{array}{l}\text { Longitude (Degrees } \\
\text { Minutes Seconds) }\end{array}$ \\
\hline 07288935 & E119 & $\begin{array}{l}\text { Steele Bayou above the Sunflower Cut-off nr } \\
\text { Redwood, MS }\end{array}$ & $32^{\circ} 27^{\prime} 53^{\prime \prime}$ & $90^{\circ} 53^{\prime} 35^{\prime \prime}$ \\
\hline 07288938 & E017 & $\begin{array}{l}\text { Steele Bayou Floodgate (Landside) nr Red- } \\
\text { wood, MS }\end{array}$ & $32^{\circ} 27^{\prime} 09^{\prime \prime}$ & $90^{\circ} 53^{\prime} 25^{\prime \prime}$ \\
\hline 0728860950 & $\mathrm{E} 123$ & $\begin{array}{l}\text { Quiver River at North Mobile Rd Nr Moore- } \\
\text { head, MS }\end{array}$ & $33^{\circ} 28^{\prime} 05^{\prime \prime}$ & $90^{\circ} 33^{\prime} 52^{\prime \prime}$ \\
\hline 0728862450 & E126 & $\begin{array}{l}\text { Big Sunflower above Bogue Phalia near } \\
\text { Darlove, MS }\end{array}$ & $33^{\circ} 15^{\prime} 15^{\prime \prime}$ & $90^{\circ} 43^{\prime} 48^{\prime \prime}$ \\
\hline 0728879790 & E116 & False River nr Onward, MS & $32^{\circ} 42^{\prime} 23^{\prime \prime}$ & $90^{\circ} 52^{\prime} 52^{\prime \prime}$ \\
\hline 325449090451700 & E122 & $\begin{array}{l}\text { Big Sunflower River below Holly Bluff Cut- } \\
\text { off nr Rolling Fork, MS }\end{array}$ & $32^{\circ} 54^{\prime} 49^{\prime \prime}$ & $90^{\circ} 45^{\prime} 17^{\prime \prime}$ \\
\hline 325616090461600 & E121 & $\begin{array}{l}\text { Big Sunflower River at Big Eddy Landing nr } \\
\text { Rolling Fork, MS }\end{array}$ & $32^{\circ} 56^{\prime} 16^{\prime \prime}$ & $90^{\circ} 46^{\prime} 16^{\prime \prime}$ \\
\hline 325901090465500 & D014 & Jaynes Bayou at Mouth nr Anguilla, MS & $32^{\circ} 58^{\prime} 56^{\prime \prime}$ & $90^{\circ} 46^{\prime} 42^{\prime \prime}$ \\
\hline 330236090420100 & $\mathrm{E} 120$ & Big Sunflower River nr Delta City, MS & $33^{\circ} 02^{\prime} 36^{\prime \prime}$ & $90^{\circ} 42^{\prime} 01^{\prime \prime}$ \\
\hline 330730090431300 & D018 & Murphy Bayou at Murphy, MS & $33^{\circ} 07^{\prime} 30^{\prime \prime}$ & $90^{\circ} 43^{\prime} 13^{\prime \prime}$ \\
\hline 331637090432700 & E125 & Big Sunflower River below Kinlock, MS & $33^{\circ} 16^{\prime} 37^{\prime \prime}$ & $90^{\circ} 43^{\prime} 27^{\prime \prime}$ \\
\hline 332749090103400 & D019 & Pelucia Creek nr Rising Sun, MS & $33^{\circ} 27^{\prime} 51^{\prime \prime}$ & $90^{\circ} 10^{\prime} 25^{\prime \prime}$ \\
\hline 333328090554800 & D029 & Clear Creek nr Stringtown, MS & $33^{\circ} 33^{\prime} 28^{\prime \prime}$ & $90^{\circ} 55^{\prime} 48^{\prime \prime}$ \\
\hline 333615090061800 & D038 & Teoc Creek nr Teoc, MS & $33^{\circ} 36^{\prime} 15^{\prime \prime}$ & $90^{\circ} 06^{\prime} 18^{\prime \prime}$ \\
\hline 334747090285000 & D028 & Wild Bill Bayou nr Drew, MS & $33^{\circ} 47^{\prime} 47^{\prime \prime}$ & $90^{\circ} 28^{\prime} 50^{\prime \prime}$ \\
\hline 335105090415300 & D017 & Mound Bayou nr Merigold, MS & $33^{\circ} 51^{\prime} 03^{\prime \prime}$ & $90^{\circ} 41^{\prime} 51^{\prime \prime}$ \\
\hline 335458090287000 & D011 & Bear Bayou nr Parchman, MS & $33^{\circ} 54^{\prime} 57^{\prime \prime}$ & $90^{\circ} 28^{\prime} 48^{\prime \prime}$ \\
\hline 335749090273300 & D013 & Home Cypress Bayou nr Rome, MS & $33^{\circ} 57^{\prime} 49^{\prime \prime}$ & $90^{\circ} 27^{\prime} 33^{\prime \prime}$ \\
\hline 335919090133600 & D007 & Opossum Bayou nr Webb, MS & $33^{\circ} 59^{\prime} 19^{\prime \prime}$ & $90^{\circ} 13^{\prime} 36^{\prime \prime}$ \\
\hline 340157090071200 & D008 & $\begin{array}{l}\text { Unnamed Tributary Panola Floodway nr } \\
\text { Charleston, MS }\end{array}$ & $34^{\circ} 01^{\prime} 57^{\prime \prime}$ & $90^{\circ} 07^{\prime} 12^{\prime \prime}$ \\
\hline 340423090210800 & D034 & Hopson Bayou at Vance, MS & $34^{\circ} 04^{\prime} 23^{\prime \prime}$ & $90^{\circ} 21^{\prime} 08^{\prime \prime}$ \\
\hline 341640090055100 & D025 & $\begin{array}{l}\text { Unnamed Tributary to Tallahatchie River nr } \\
\text { Locke Station, MS }\end{array}$ & $34^{\circ} 16^{\prime} 39^{\prime \prime}$ & $90^{\circ} 05^{\prime} 52^{\prime \prime}$ \\
\hline 341701090113300 & D006 & Ash Log Bayou nr Bobo, MS & $34^{\circ} 17^{\prime} 01^{\prime \prime}$ & $90^{\circ} 11^{\prime} 33^{\prime \prime}$ \\
\hline 344516090164400 & D005 & Floyd Bayou, nr Green River, MS & $34^{\circ} 45^{\prime} 16^{\prime \prime}$ & $90^{\circ} 16^{\prime} 44^{\prime \prime}$ \\
\hline
\end{tabular}


Table 2. Physical and chemical properties measured in the Mississippi Alluvial Plain ecoregion, northwestern Mississippi, September-0ctober 2007.

[FNU, formazin nephelometric units; NTU, nephelometric turbidity units; mg/L, milligrams per liter; \%, percent; $\mu \mathrm{S} / \mathrm{cm}$, microsiemens per centimeter; ${ }^{\circ} \mathrm{C}$, degrees Celsius; $\mathrm{ft} / \mathrm{s}$, cubic foot per second; $\mathrm{Cl}$, chloride; $\mathrm{N}$, nitrogen; $\mathrm{P}$, phosphorus; $\mu \mathrm{g} / \mathrm{L}$, micrograms per liter]

\begin{tabular}{|c|c|c|}
\hline 48-hour continuous measurements & $\begin{array}{l}\text { Instantaneous field physical-chemical } \\
\text { water measurements }\end{array}$ & $\begin{array}{l}\text { Instantaneous water samples } \\
\text { for laboratory analysis }\end{array}$ \\
\hline Turbidity (FNU) & Discharge $\left(\mathrm{ft}^{3} / \mathrm{s}\right)$ & Alkalinity as $\mathrm{CaCO}_{3}$ \\
\hline Dissolved oxygen $(\mathrm{mg} / \mathrm{L})$ & Turbidity (NTU) & Chloride $(\mathrm{mg} / \mathrm{L}$ as $\mathrm{Cl})$ \\
\hline Dissolved oxygen (\% Saturation) & Dissolved oxygen $(\mathrm{mg} / \mathrm{L})$ & Total suspended solids $(\mathrm{mg} / \mathrm{L})$ \\
\hline $\mathrm{pH}$ (standard units) & $\mathrm{pH}$ (standard units) & Ammonia (mg/L as N) \\
\hline Specific conductance $(\mu \mathrm{S} / \mathrm{cm})$ & Specific conductance $(\mu \mathrm{S} / \mathrm{cm})$ & Nitrogen, total Kjeldahl (mg/L as $\mathrm{N})$ \\
\hline \multirow[t]{7}{*}{ Water temperature $\left({ }^{\circ} \mathrm{C}\right)$} & Water temperature $\left({ }^{\circ} \mathrm{C}\right)$ & Nitrite plus nitrate $(\mathrm{mg} / \mathrm{L}$ as $\mathrm{N})$ \\
\hline & & Orthophosphate (mg/L as $\mathrm{P}$ ) \\
\hline & & Total phosphorus ( $\mathrm{mg} / \mathrm{L}$ as $\mathrm{P}$ ) \\
\hline & & Total organic carbon $(\mathrm{mg} / \mathrm{L})$ \\
\hline & & Chemical oxygen demand $(\mathrm{mg} / \mathrm{L})$ \\
\hline & & Chlorophyll a $(\mu \mathrm{g} / \mathrm{L})$ \\
\hline & & Total suspended sediment $(\mathrm{mg} / \mathrm{L})$ \\
\hline
\end{tabular}




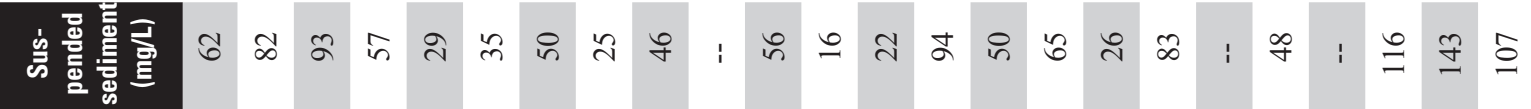

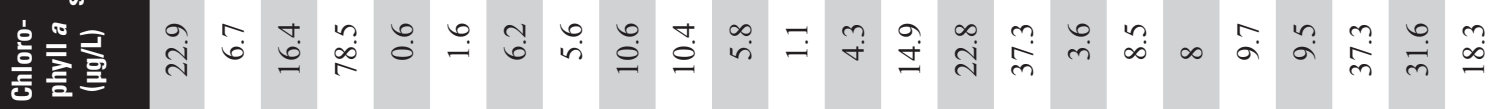

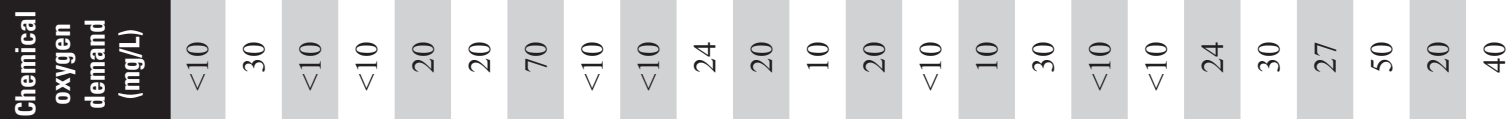

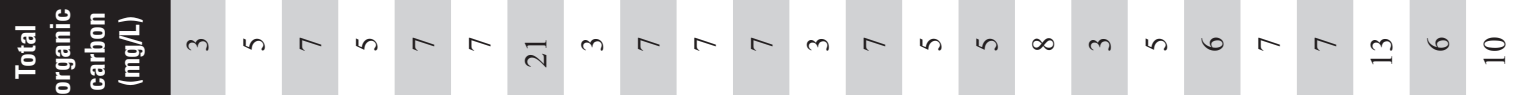

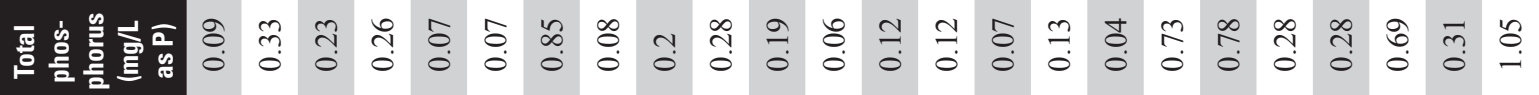

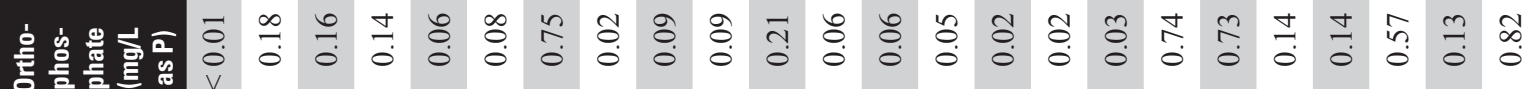

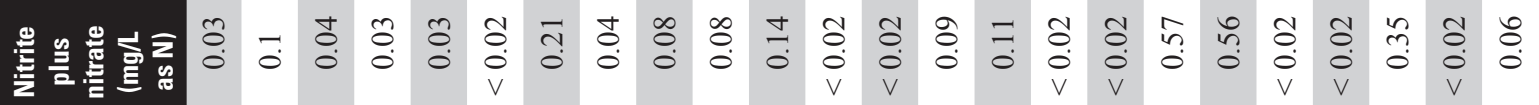

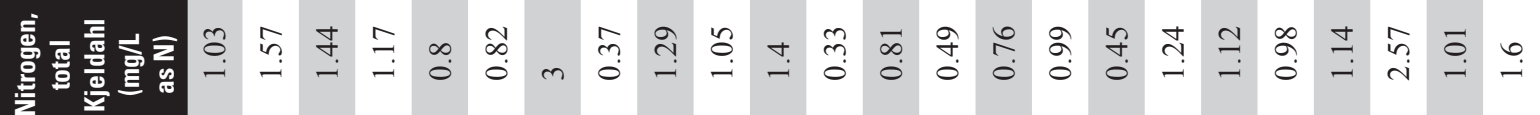

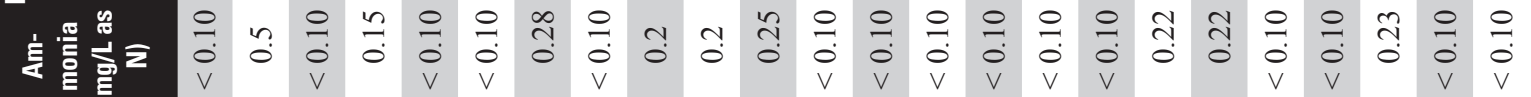

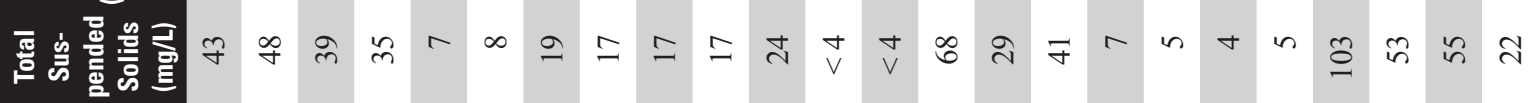

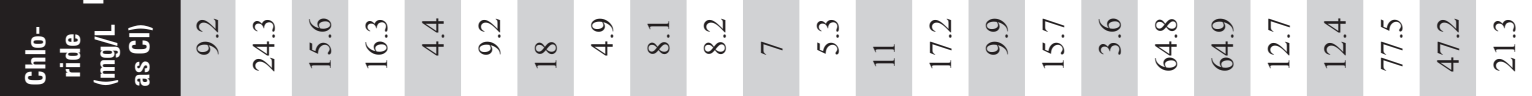

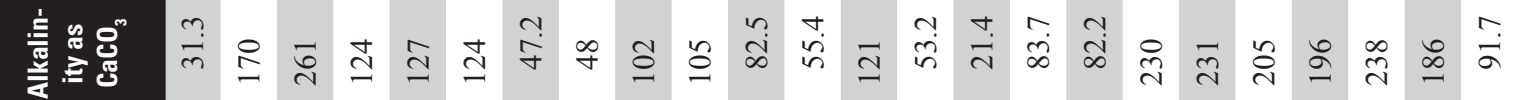

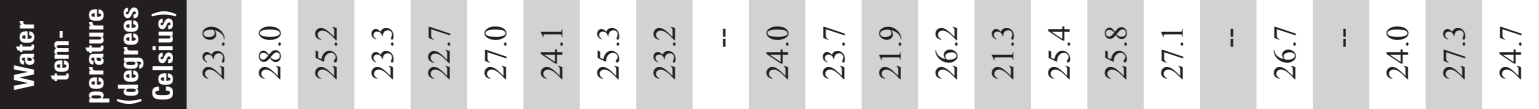

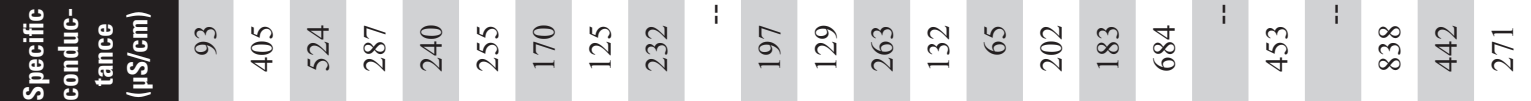

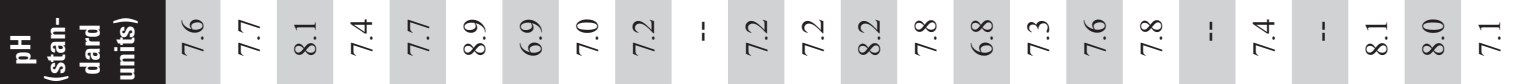

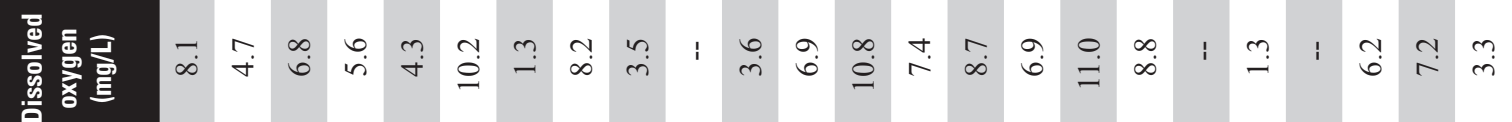
它焉

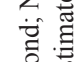

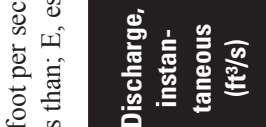

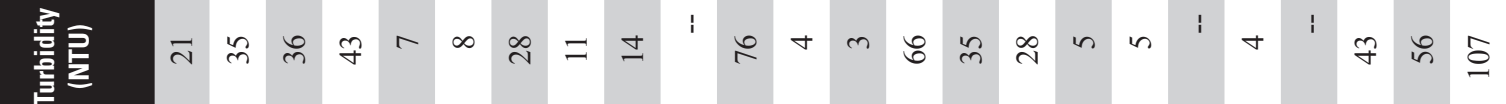

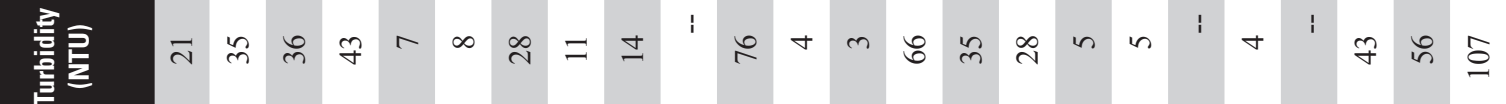
o.

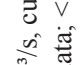

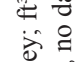

垔 亘产言

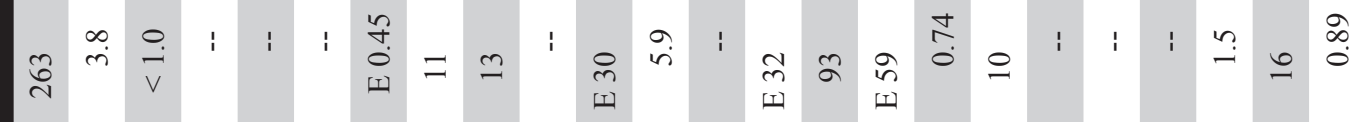

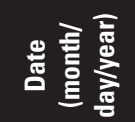

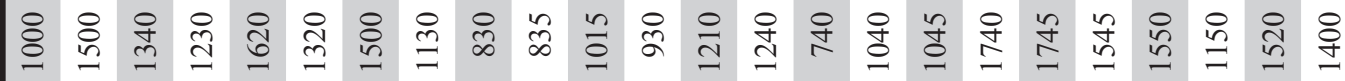

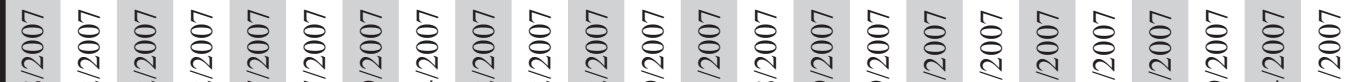

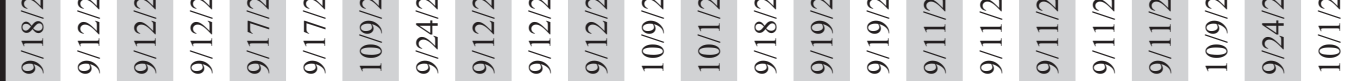




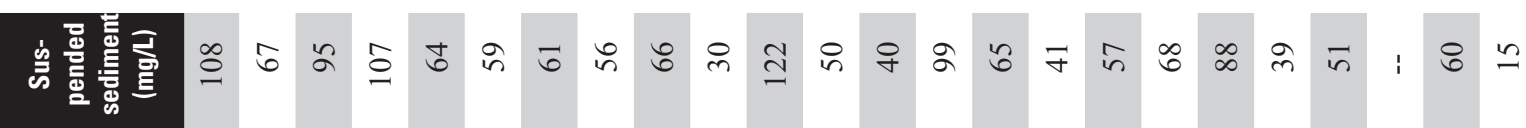

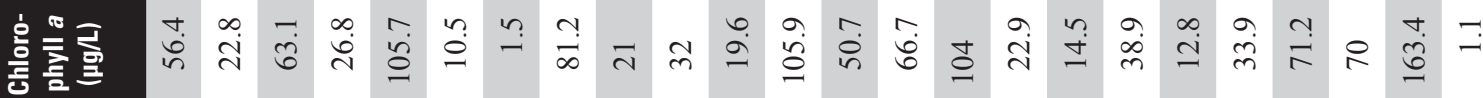

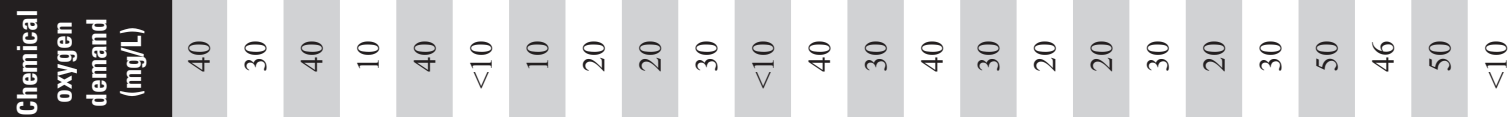

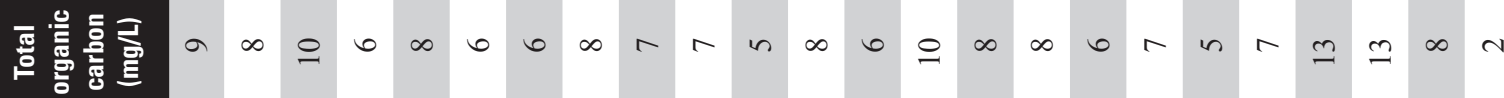

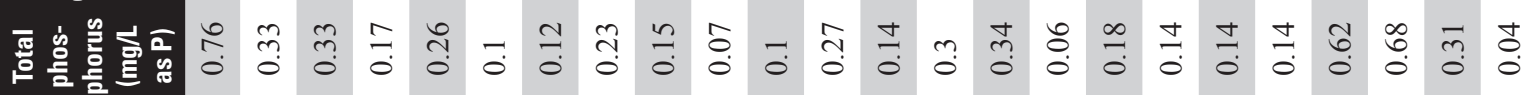

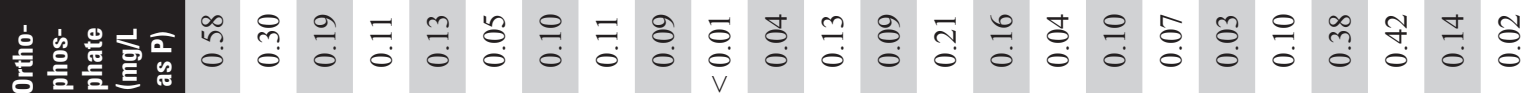

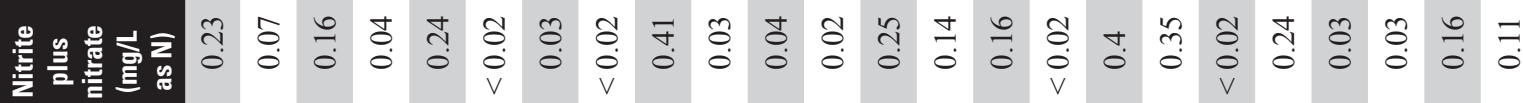

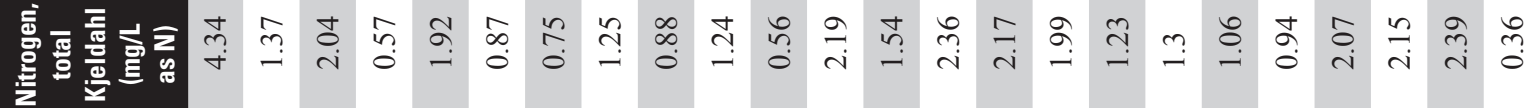

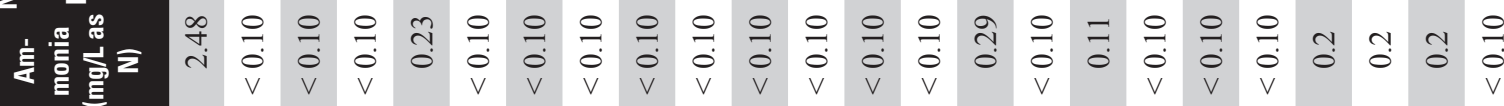

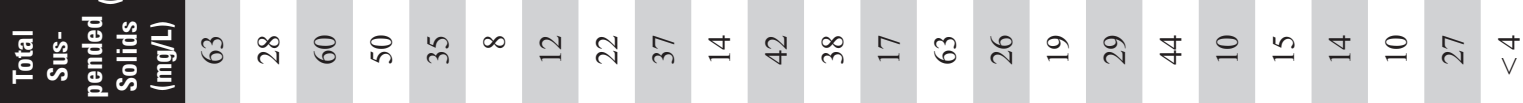

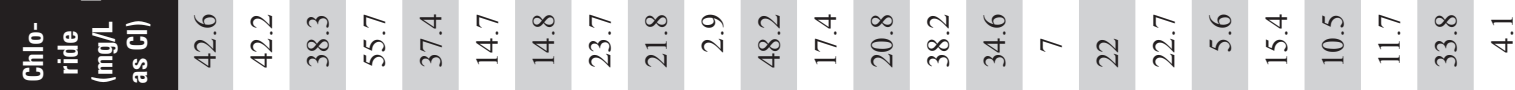

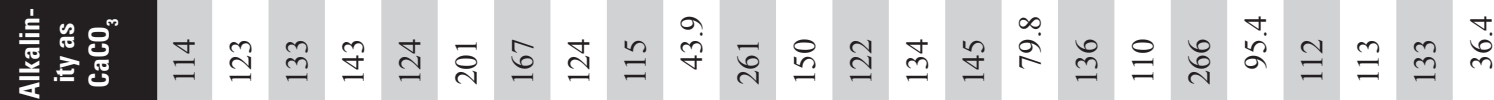

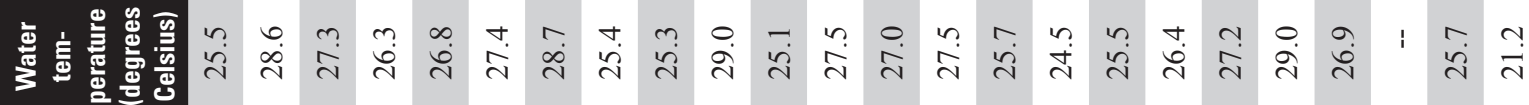

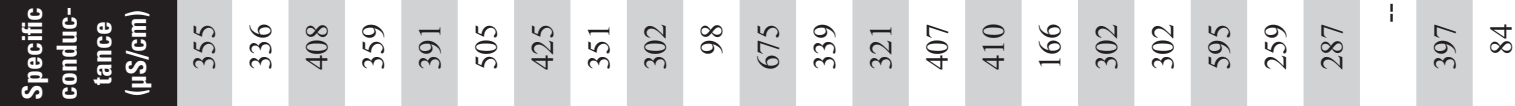

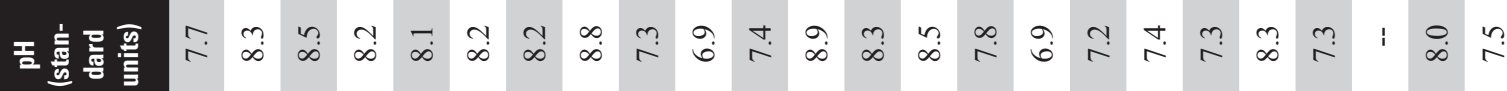
噌

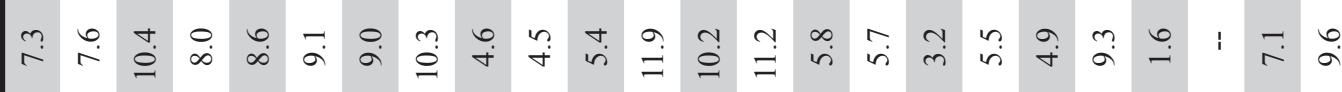
蛋 \& $\bar{m} \frac{1}{2}$ in

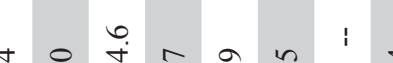

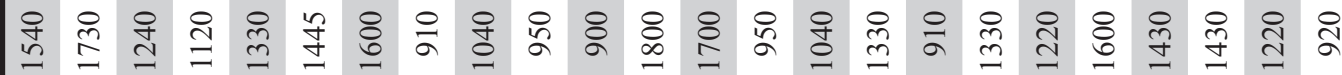

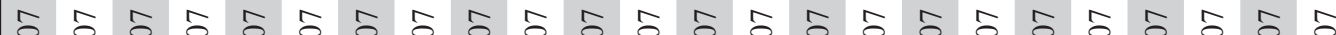

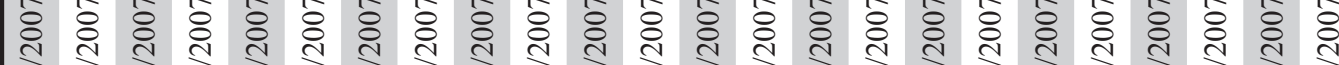

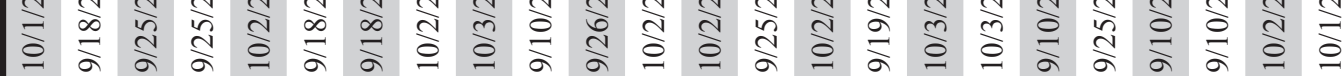




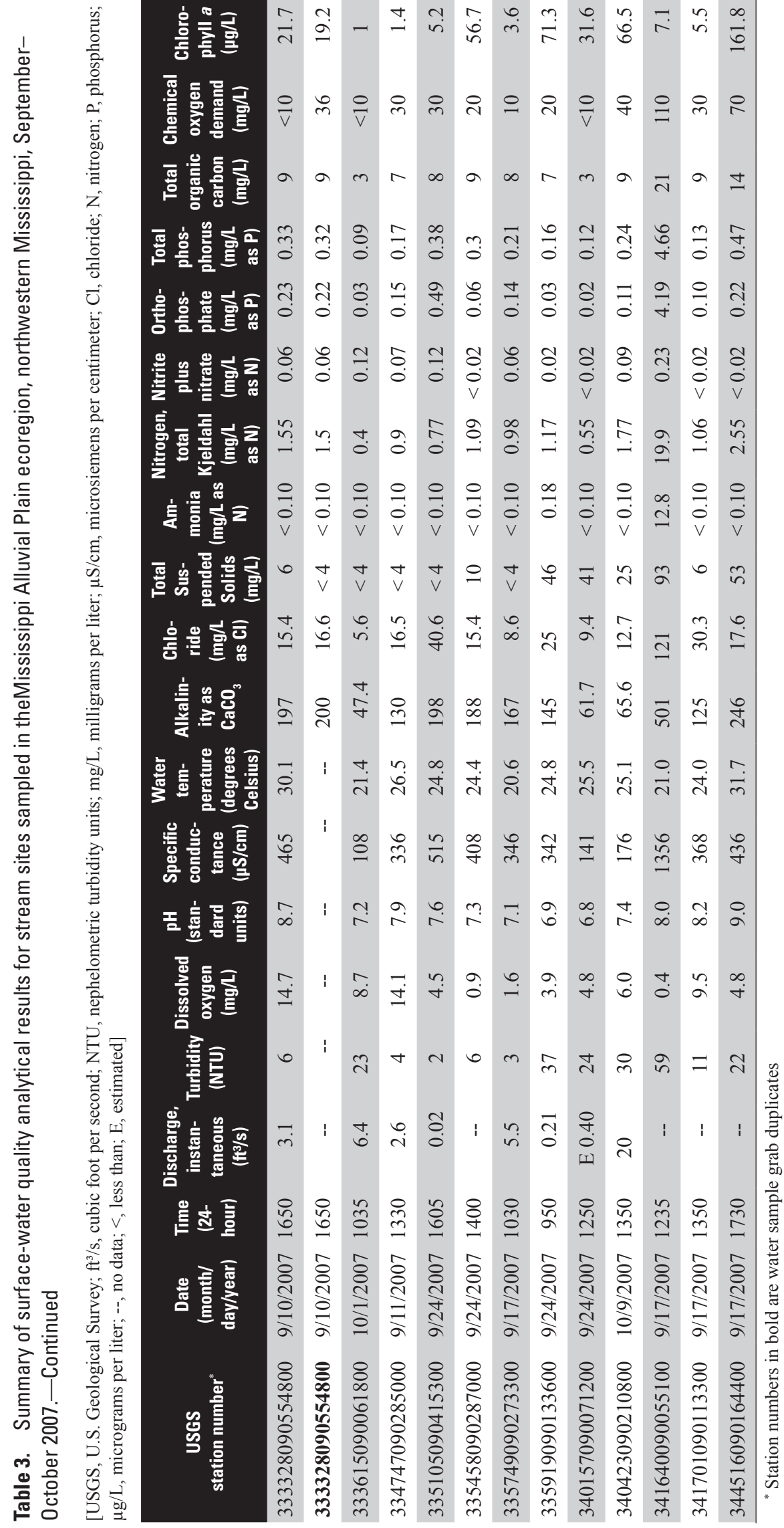

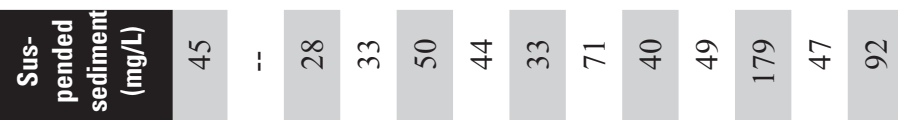

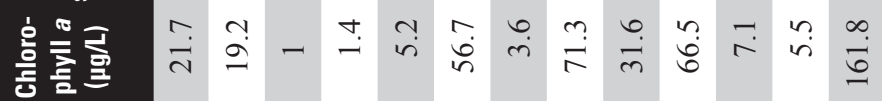

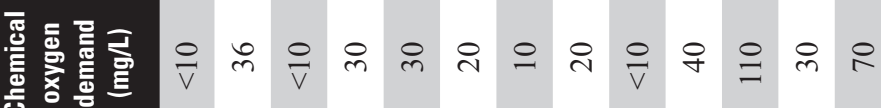

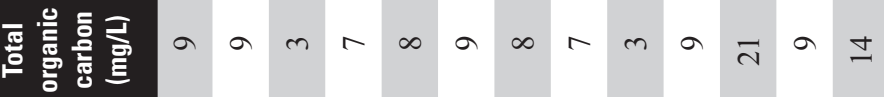

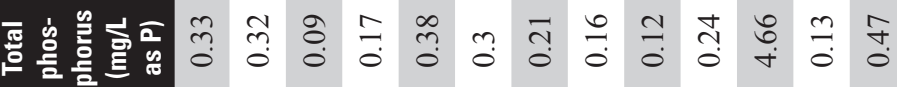

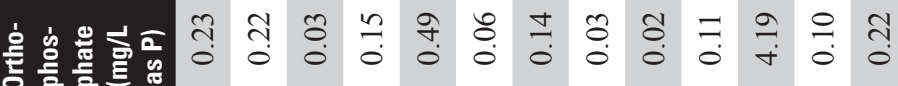

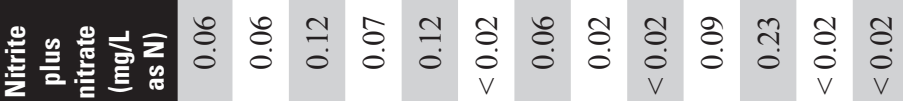

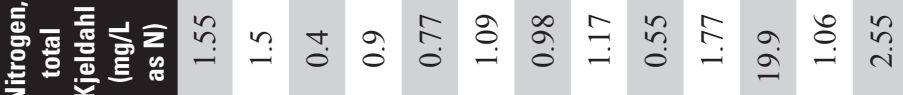

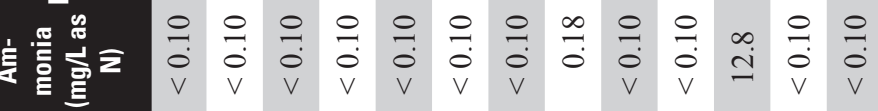

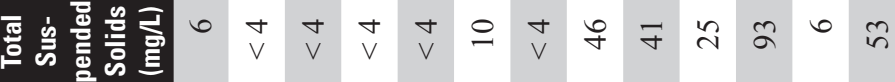

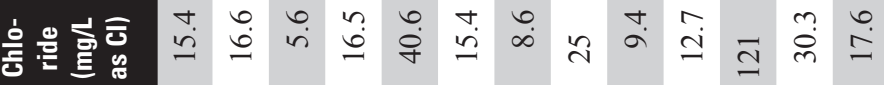

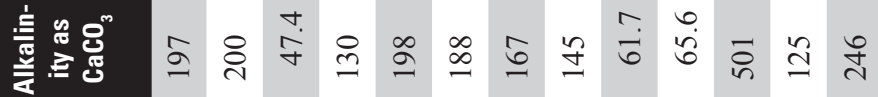

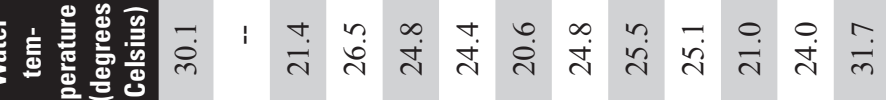

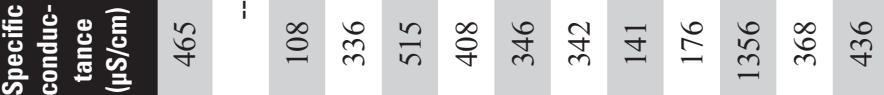

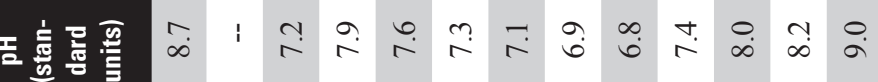

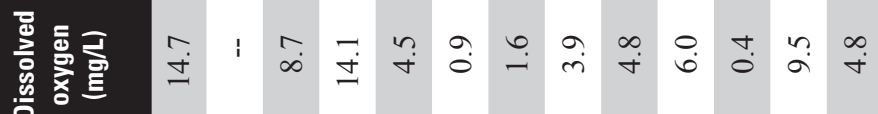

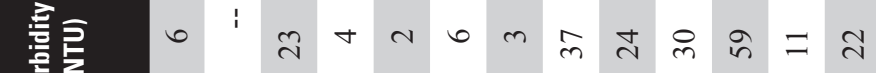

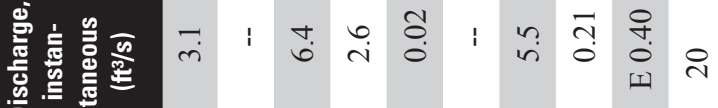
音㤩豆

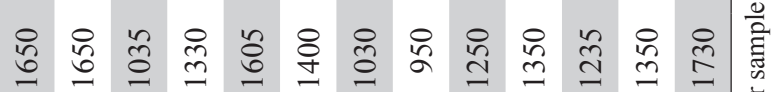

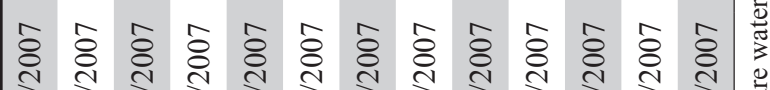
命

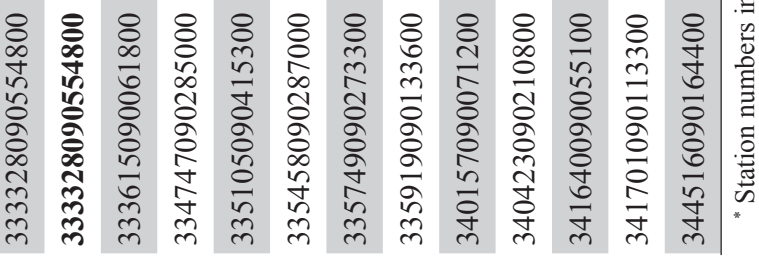


Table 4. Physical property data recorded at 30-minute intervals for station 07279836 Ark Bayou near Tibbs, Mississippi, September 17-19, 2007.

[ $\mu \mathrm{S} / \mathrm{cm}$, microsiemens per centimeter; \%sat, percent saturation; $\mathrm{mg} / \mathrm{L}$, milligrams per liter]

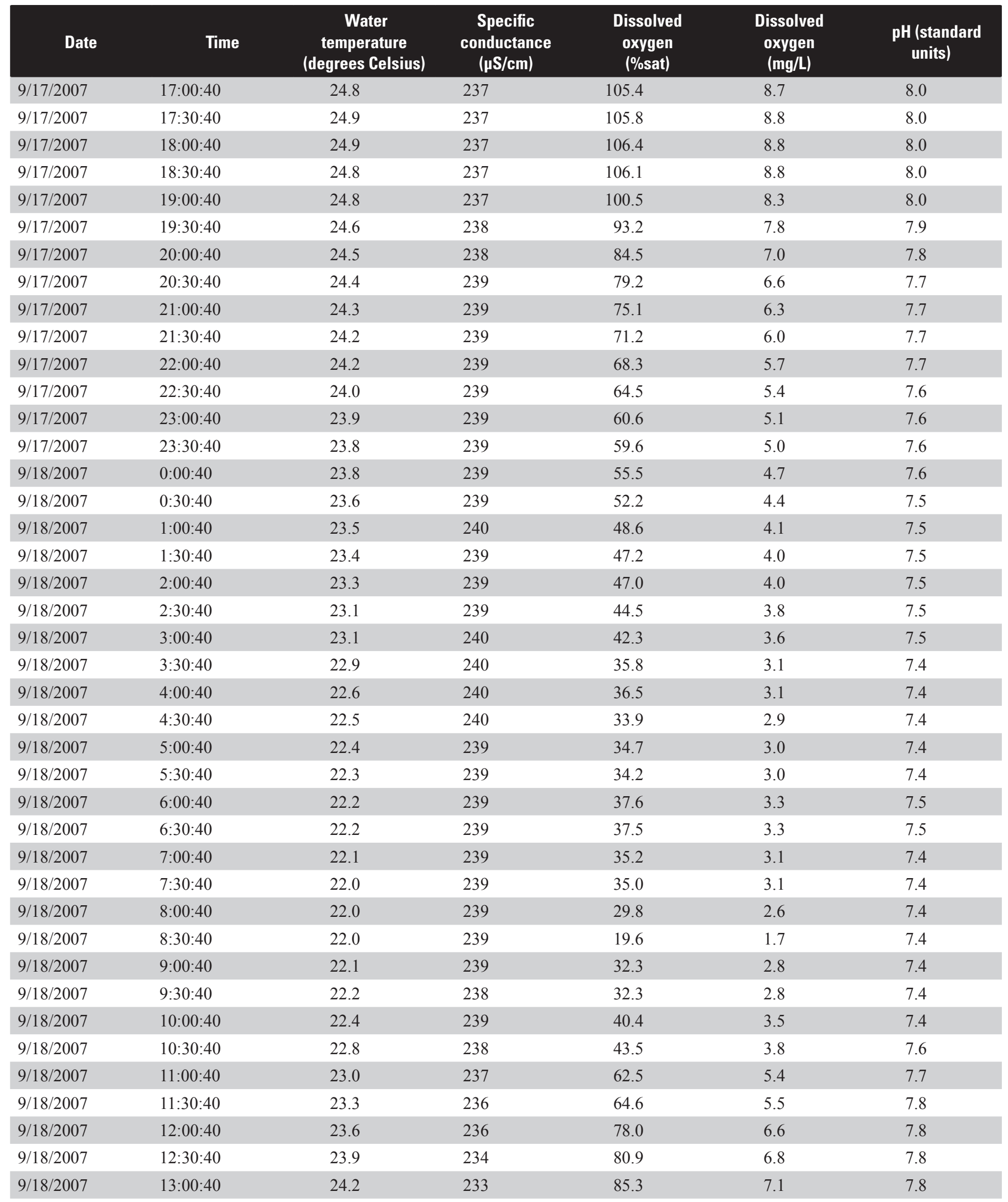


Table 4. Physical property data recorded at 30-minute intervals for station 07279836 Ark Bayou near Tibbs, Mississippi, September 17-19, 2007.-Continued

$[\mu \mathrm{S} / \mathrm{cm}$, microsiemens per centimeter; \%sat, percent saturation; $\mathrm{mg} / \mathrm{L}$, milligrams per liter]

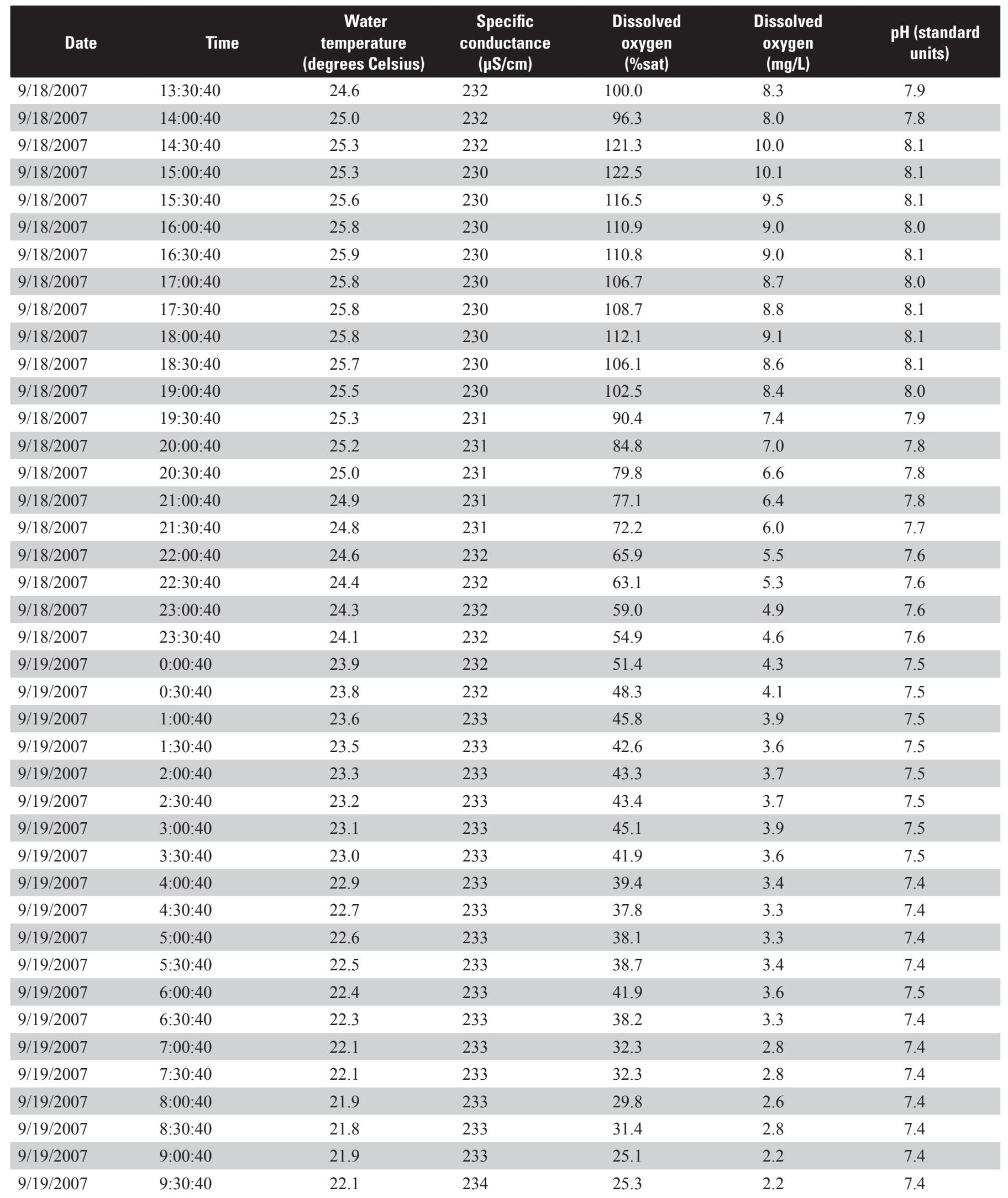


Table 4. Physical property data recorded at 30-minute intervals for station 07279836 Ark Bayou near Tibbs, Mississippi, September 17-19, 2007.-Continued

$[\mu \mathrm{S} / \mathrm{cm}$, microsiemens per centimeter; \%sat, percent saturation; $\mathrm{mg} / \mathrm{L}$, milligrams per liter]

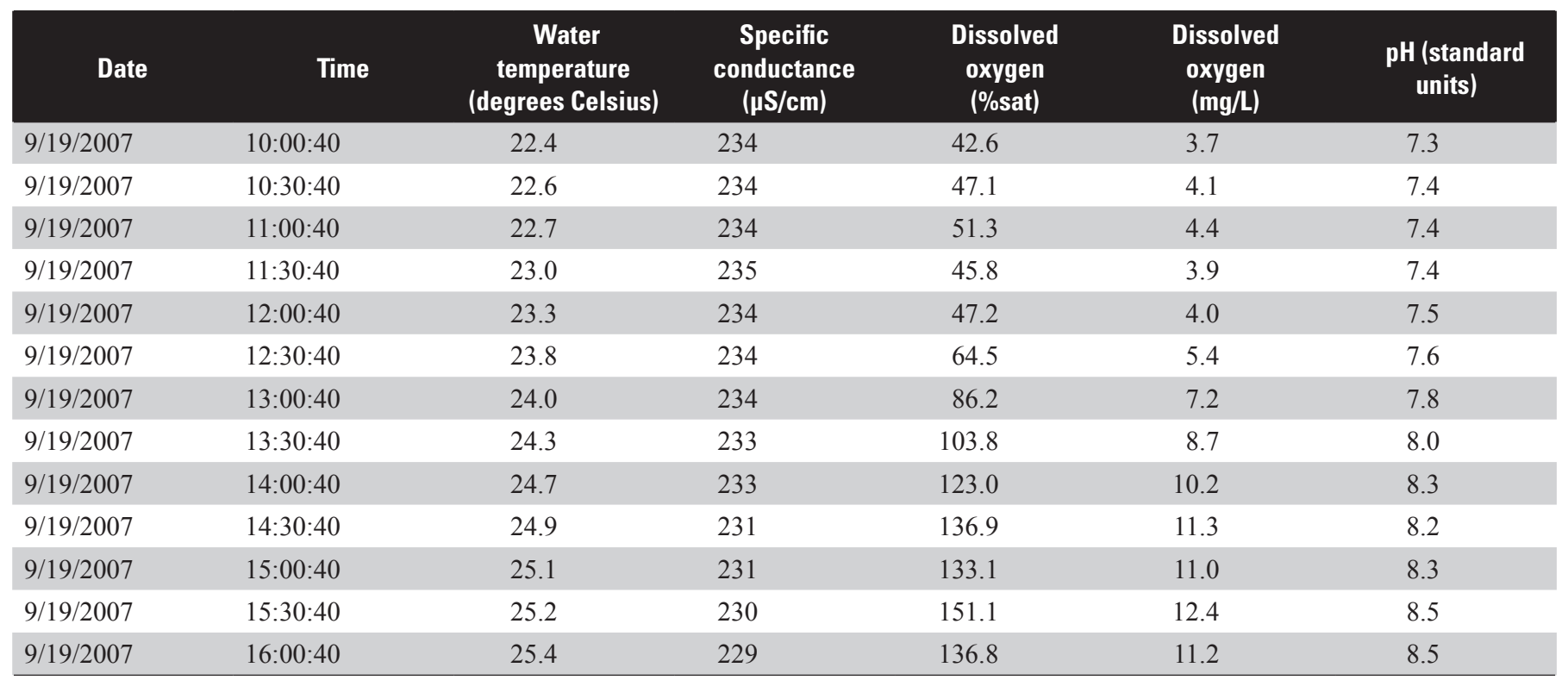


Table 5. Physical property data recorded at 30-minute intervals for station 07279937 David Bayou near Sledge, Mississippi, September 17-19, 2007

[ $\mu \mathrm{S} / \mathrm{cm}$, microsiemens per centimeter; \%sat, percent saturation; $\mathrm{mg} / \mathrm{L}$, milligrams per liter]

\begin{tabular}{|c|c|c|c|c|c|c|}
\hline Date & Time & $\begin{array}{c}\text { Water } \\
\text { temperature } \\
\text { (degrees Celsius) }\end{array}$ & $\begin{array}{c}\text { Specific } \\
\text { conductance } \\
\text { (pS/cm) }\end{array}$ & $\begin{array}{l}\text { Dissolved } \\
\text { oxygen } \\
\text { (\%sat) }\end{array}$ & $\begin{array}{l}\text { Dissolved } \\
\text { oxygen } \\
\text { (mg/L) }\end{array}$ & $\begin{array}{l}\text { pH (standard } \\
\text { units) }\end{array}$ \\
\hline $9 / 17 / 2007$ & $15: 31: 00$ & 27.4 & 245 & 167 & 13.1 & 9.0 \\
\hline $9 / 17 / 2007$ & $16: 01: 00$ & 27.8 & 230 & 182 & 14.3 & 9.1 \\
\hline $9 / 17 / 2007$ & $16: 31: 00$ & 28.1 & 225 & 189 & 14.8 & 9.2 \\
\hline $9 / 17 / 2007$ & 17:01:00 & 28.2 & 218 & 196 & 15.3 & 9.2 \\
\hline $9 / 17 / 2007$ & $17: 31: 00$ & 28.3 & 231 & 199 & 15.5 & 9.2 \\
\hline $9 / 17 / 2007$ & 18:01:00 & 28.2 & 223 & 203 & 15.8 & 9.3 \\
\hline $9 / 17 / 2007$ & 18:31:00 & 28.1 & 220 & 205 & 16.0 & 9.3 \\
\hline $9 / 17 / 2007$ & 19:01:00 & 28.0 & 224 & 207 & 16.2 & 9.3 \\
\hline $9 / 17 / 2007$ & 19:31:00 & 27.7 & 228 & 208 & 16.4 & 9.3 \\
\hline $9 / 17 / 2007$ & 20:01:00 & 27.4 & 227 & 207 & 16.4 & 9.3 \\
\hline $9 / 17 / 2007$ & 20:31:00 & 27.2 & 221 & 204 & 16.2 & 9.3 \\
\hline $9 / 17 / 2007$ & 21:01:00 & 26.8 & 230 & 202 & 16.1 & 9.3 \\
\hline $9 / 17 / 2007$ & $21: 31: 00$ & 26.6 & 230 & 193 & 15.5 & 9.3 \\
\hline $9 / 17 / 2007$ & $22: 01: 00$ & 26.3 & 230 & 186 & 15.0 & 9.3 \\
\hline $9 / 17 / 2007$ & $22: 31: 00$ & 26.0 & 233 & 178 & 14.5 & 9.3 \\
\hline $9 / 17 / 2007$ & $23: 01: 00$ & 25.7 & 235 & 170 & 13.9 & 9.3 \\
\hline $9 / 17 / 2007$ & $23: 31: 00$ & 25.4 & 236 & 162 & 13.3 & 9.3 \\
\hline $9 / 18 / 2007$ & 0:01:00 & 25.2 & 238 & 153 & 12.6 & 9.2 \\
\hline $9 / 18 / 2007$ & $0: 31: 00$ & 24.9 & 239 & 144 & 11.9 & 9.2 \\
\hline $9 / 18 / 2007$ & 1:01:00 & 24.7 & 241 & 135 & 11.2 & 9.1 \\
\hline $9 / 18 / 2007$ & 1:31:00 & 24.4 & 242 & 126 & 10.5 & 9.1 \\
\hline $9 / 18 / 2007$ & 2:01:00 & 24.2 & 244 & 118 & 9.8 & 9.0 \\
\hline $9 / 18 / 2007$ & $2: 31: 00$ & 24.0 & 245 & 110 & 9.3 & 9.0 \\
\hline $9 / 18 / 2007$ & $3: 01: 00$ & 23.8 & 247 & 103 & 8.7 & 8.9 \\
\hline $9 / 18 / 2007$ & $3: 31: 00$ & 23.7 & 249 & 96 & 8.2 & 8.9 \\
\hline $9 / 18 / 2007$ & 4:01:00 & 23.5 & 251 & 91 & 7.7 & 8.8 \\
\hline $9 / 18 / 2007$ & $4: 31: 00$ & 23.4 & 252 & 85 & 7.3 & 8.7 \\
\hline $9 / 18 / 2007$ & 5:01:00 & 23.2 & 253 & 80 & 6.8 & 8.6 \\
\hline $9 / 18 / 2007$ & $5: 31: 00$ & 23.1 & 255 & 75 & 6.4 & 8.6 \\
\hline $9 / 18 / 2007$ & $6: 01: 00$ & 22.9 & 257 & 71 & 6.1 & 8.5 \\
\hline $9 / 18 / 2007$ & $6: 31: 00$ & 22.8 & 257 & 66 & 5.7 & 8.4 \\
\hline $9 / 18 / 2007$ & 7:01:00 & 22.7 & 259 & 62 & 5.4 & 8.3 \\
\hline $9 / 18 / 2007$ & $7: 31: 00$ & 22.6 & 262 & 58 & 5.0 & 8.2 \\
\hline $9 / 18 / 2007$ & 8:01:00 & 22.5 & 262 & 56 & 4.8 & 8.1 \\
\hline $9 / 18 / 2007$ & $8: 31: 00$ & 22.5 & 265 & 54 & 4.7 & 8.1 \\
\hline $9 / 18 / 2007$ & 9:01:00 & 22.6 & 266 & 55 & 4.8 & 8.1 \\
\hline $9 / 18 / 2007$ & $9: 31: 00$ & 22.8 & 266 & 59 & 5.1 & 8.1 \\
\hline $9 / 18 / 2007$ & 10:01:00 & 23.0 & 266 & 64 & 5.4 & 8.1 \\
\hline $9 / 18 / 2007$ & 10:31:00 & 23.4 & 266 & 70 & 6.0 & 8.2 \\
\hline $9 / 18 / 2007$ & 11:01:00 & 23.7 & 267 & 76 & 6.4 & 8.2 \\
\hline
\end{tabular}


Table 5. Physical property data recorded at 30-minute intervals for station 07279937 David Bayou near Sledge, Mississippi, September 17-19, 2007.-Continued

$[\mu \mathrm{S} / \mathrm{cm}$, microsiemens per centimeter; \%sat, percent saturation; $\mathrm{mg} / \mathrm{L}$, milligrams per liter]

\begin{tabular}{|c|c|c|c|c|c|c|}
\hline Date & Time & $\begin{array}{c}\text { Water } \\
\text { temperature } \\
\text { (degrees Celsius) }\end{array}$ & $\begin{array}{c}\text { Specific } \\
\text { conductance } \\
\text { (pS/cm) }\end{array}$ & $\begin{array}{l}\text { Dissolved } \\
\text { oxygen } \\
\text { (\%sat) }\end{array}$ & $\begin{array}{l}\text { Dissolved } \\
\text { oxygen } \\
\text { (mg/L) }\end{array}$ & $\begin{array}{l}\text { pH (standard } \\
\text { units) }\end{array}$ \\
\hline $9 / 18 / 2007$ & $11: 31: 00$ & 24.1 & 267 & 83 & 6.9 & 8.3 \\
\hline $9 / 18 / 2007$ & 12:01:00 & 24.5 & 268 & 90 & 7.5 & 8.4 \\
\hline $9 / 18 / 2007$ & $12: 31: 00$ & 25.0 & 268 & 101 & 8.3 & 8.5 \\
\hline $9 / 18 / 2007$ & 13:01:00 & 25.5 & 268 & 113 & 9.3 & 8.6 \\
\hline $9 / 18 / 2007$ & $13: 31: 00$ & 26.0 & 266 & 127 & 10.3 & 8.7 \\
\hline $9 / 18 / 2007$ & 14:01:00 & 26.6 & 266 & 138 & 11.1 & 8.8 \\
\hline $9 / 18 / 2007$ & $14: 31: 00$ & 27.0 & 264 & 155 & 12.3 & 8.9 \\
\hline $9 / 18 / 2007$ & $15: 01: 00$ & 27.5 & 263 & 171 & 13.5 & 8.9 \\
\hline $9 / 18 / 2007$ & $15: 31: 00$ & 27.9 & 260 & 186 & 14.5 & 9.0 \\
\hline $9 / 18 / 2007$ & $16: 01: 00$ & 28.1 & 259 & 198 & 15.4 & 9.1 \\
\hline $9 / 18 / 2007$ & $16: 31: 00$ & 28.3 & 256 & 205 & 16.0 & 9.2 \\
\hline $9 / 18 / 2007$ & 17:01:00 & 28.4 & 253 & 213 & 16.5 & 9.2 \\
\hline $9 / 18 / 2007$ & $17: 31: 00$ & 28.5 & 251 & 218 & 16.9 & 9.3 \\
\hline $9 / 18 / 2007$ & 18:01:00 & 28.4 & 250 & 222 & 17.3 & 9.3 \\
\hline $9 / 18 / 2007$ & $18: 31: 00$ & 28.2 & 249 & 220 & 17.1 & 9.3 \\
\hline $9 / 18 / 2007$ & 19:01:00 & 28.1 & 247 & 213 & 16.6 & 9.3 \\
\hline $9 / 18 / 2007$ & $19: 31: 00$ & 27.8 & 245 & 211 & 16.6 & 9.3 \\
\hline $9 / 18 / 2007$ & 20:01:00 & 27.6 & 244 & 208 & 16.4 & 9.3 \\
\hline $9 / 18 / 2007$ & $20: 31: 00$ & 27.3 & 242 & 206 & 16.3 & 9.3 \\
\hline $9 / 18 / 2007$ & 21:01:00 & 27.0 & 242 & 204 & 16.2 & 9.3 \\
\hline $9 / 18 / 2007$ & $21: 31: 00$ & 26.7 & 242 & 200 & 16.0 & 9.3 \\
\hline $9 / 18 / 2007$ & $22: 01: 00$ & 26.4 & 241 & 196 & 15.8 & 9.3 \\
\hline $9 / 18 / 2007$ & $22: 31: 00$ & 26.1 & 241 & 191 & 15.4 & 9.3 \\
\hline $9 / 18 / 2007$ & 23:01:00 & 25.8 & 242 & 185 & 15.1 & 9.3 \\
\hline $9 / 18 / 2007$ & $23: 31: 00$ & 25.5 & 242 & 179 & 14.7 & 9.2 \\
\hline $9 / 19 / 2007$ & 0:01:00 & 25.2 & 243 & 172 & 14.2 & 9.2 \\
\hline $9 / 19 / 2007$ & $0: 31: 00$ & 24.9 & 244 & 164 & 13.6 & 9.2 \\
\hline 9/19/2007 & 1:01:00 & 24.6 & 245 & 156 & 13.0 & 9.1 \\
\hline $9 / 19 / 2007$ & $1: 31: 00$ & 24.4 & 246 & 147 & 12.3 & 9.1 \\
\hline $9 / 19 / 2007$ & $2: 01: 00$ & 24.1 & 247 & 139 & 11.6 & 9.0 \\
\hline $9 / 19 / 2007$ & $2: 31: 00$ & 23.9 & 250 & 130 & 10.9 & 8.9 \\
\hline $9 / 19 / 2007$ & $3: 01: 00$ & 23.6 & 252 & 121 & 10.2 & 8.9 \\
\hline 9/19/2007 & $3: 31: 00$ & 23.4 & 253 & 113 & 9.6 & 8.8 \\
\hline 9/19/2007 & 4:01:00 & 23.2 & 255 & 106 & 9.0 & 8.8 \\
\hline $9 / 19 / 2007$ & $4: 31: 00$ & 23.0 & 256 & 99 & 8.5 & 8.7 \\
\hline $9 / 19 / 2007$ & $5: 01: 00$ & 22.8 & 259 & 93 & 8.0 & 8.6 \\
\hline $9 / 19 / 2007$ & $5: 31: 00$ & 22.6 & 259 & 87 & 7.5 & 8.6 \\
\hline 9/19/2007 & 6:01:00 & 22.4 & 262 & 82 & 7.1 & 8.5 \\
\hline $9 / 19 / 2007$ & $6: 31: 00$ & 22.2 & 264 & 76 & 6.6 & 8.4 \\
\hline 9/19/2007 & 7:01:00 & 22.0 & 266 & 71 & 6.2 & 8.3 \\
\hline
\end{tabular}


Table 5. Physical property data recorded at 30-minute intervals for station 07279937 David Bayou near Sledge, Mississippi, September 17-19, 2007.-Continued

$[\mu \mathrm{S} / \mathrm{cm}$, microsiemens per centimeter; \%sat, percent saturation; $\mathrm{mg} / \mathrm{L}$, milligrams per liter]

\begin{tabular}{|c|c|c|c|c|c|c|}
\hline Date & Time & $\begin{array}{c}\text { Water } \\
\text { temperature } \\
\text { (degrees Celsius) }\end{array}$ & $\begin{array}{c}\text { Specific } \\
\text { conductance } \\
\text { (pS/cm) }\end{array}$ & $\begin{array}{l}\text { Dissolved } \\
\text { oxygen } \\
\text { (\%sat) }\end{array}$ & $\begin{array}{l}\text { Dissolved } \\
\text { oxygen } \\
\text { (mg/L) }\end{array}$ & $\begin{array}{l}\text { pH (standard } \\
\text { units) }\end{array}$ \\
\hline 9/19/2007 & $7: 31: 00$ & 21.8 & 268 & 66 & 5.8 & 8.2 \\
\hline $9 / 19 / 2007$ & 8:01:00 & 21.7 & 269 & 63 & 5.5 & 8.2 \\
\hline $9 / 19 / 2007$ & $8: 31: 00$ & 21.7 & 271 & 60 & 5.2 & 8.1 \\
\hline 9/19/2007 & 9:01:00 & 21.8 & 271 & 57 & 5.0 & 8.1 \\
\hline $9 / 19 / 2007$ & $9: 31: 00$ & 22.0 & 273 & 58 & 5.1 & 8.1 \\
\hline 9/19/2007 & 10:01:00 & 22.3 & 274 & 60 & 5.2 & 8.1 \\
\hline $9 / 19 / 2007$ & $10: 31: 00$ & 22.7 & 276 & 63 & 5.4 & 8.1 \\
\hline 9/19/2007 & 11:01:00 & 23.2 & 276 & 67 & 5.7 & 8.2 \\
\hline $9 / 19 / 2007$ & $11: 31: 00$ & 23.8 & 276 & 73 & 6.2 & 8.3 \\
\hline 9/19/2007 & $12: 01: 00$ & 24.2 & 277 & 78 & 6.6 & 8.3 \\
\hline 9/19/2007 & $12: 31: 00$ & 24.8 & 277 & 87 & 7.2 & 8.4 \\
\hline $9 / 19 / 2007$ & 13:01:00 & 25.1 & 278 & 97 & 8.0 & 8.5 \\
\hline $9 / 19 / 2007$ & $13: 31: 00$ & 25.8 & 278 & 109 & 8.9 & 8.6 \\
\hline $9 / 19 / 2007$ & 14:01:00 & 26.5 & 277 & 124 & 9.9 & 8.7 \\
\hline $9 / 19 / 2007$ & $14: 31: 00$ & 27.1 & 276 & 137 & 10.8 & 8.8 \\
\hline 9/19/2007 & $15: 01: 00$ & 27.6 & 275 & 149 & 11.7 & 8.8 \\
\hline $9 / 19 / 2007$ & $15: 31: 00$ & 27.9 & 274 & 159 & 12.4 & 8.9 \\
\hline
\end{tabular}


Table 6. Physical property data recorded at 30-minute intervals for station 07279970 Bobo Bayou at Bobo, Mississippi, October 4-9, 2007.

$[\mu \mathrm{S} / \mathrm{cm}$, microsiemens per centimeter; \%sat, percent saturation; $\mathrm{mg} / \mathrm{L}$, milligrams per liter]

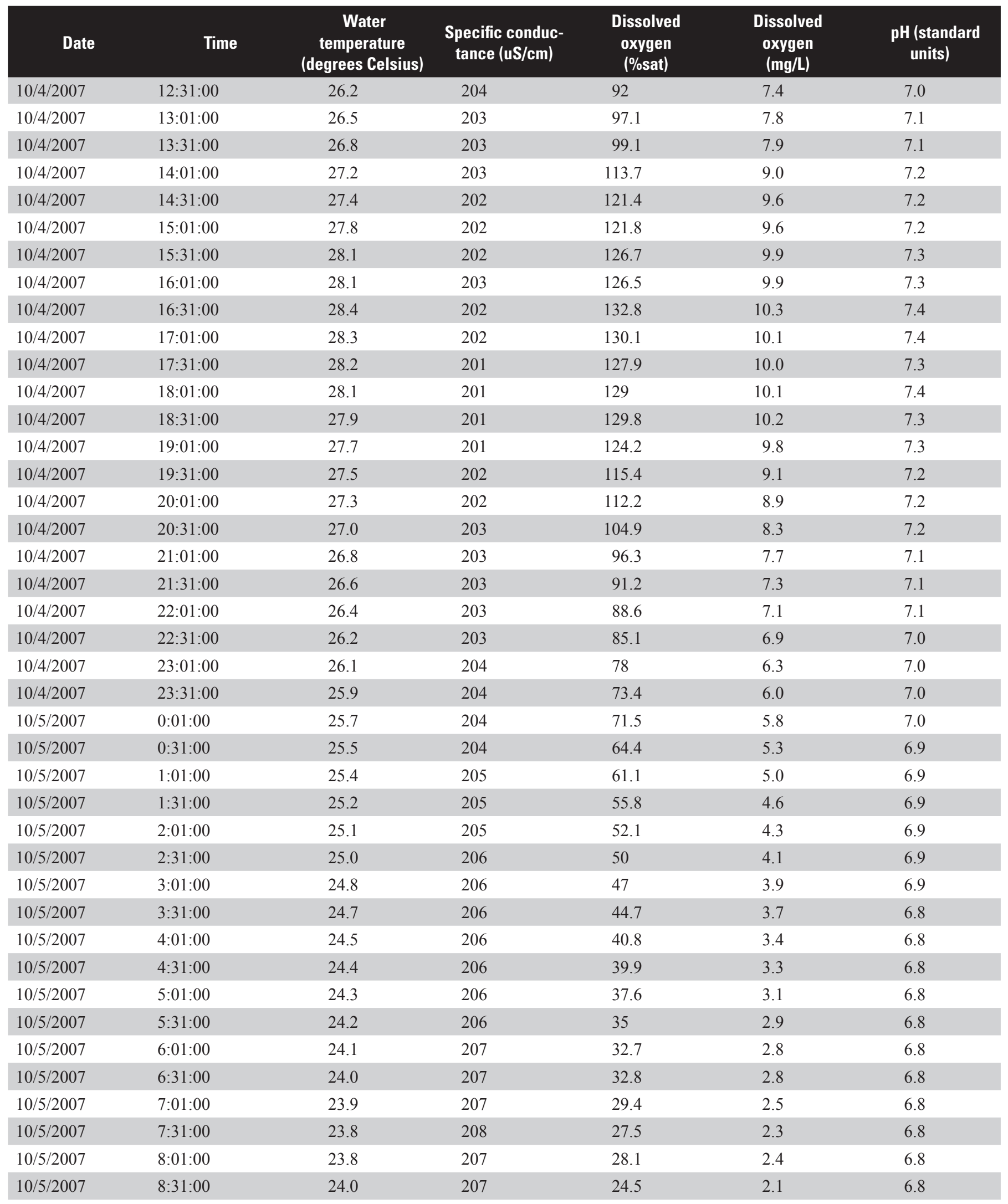


Table 6. Physical property data recorded at 30-minute intervals for station 07279970 Bobo Bayou at Bobo, Mississippi, October 4-9, 2007.-Continued

$[\mu \mathrm{S} / \mathrm{cm}$, microsiemens per centimeter; \%sat, percent saturation; $\mathrm{mg} / \mathrm{L}$, milligrams per liter $]$

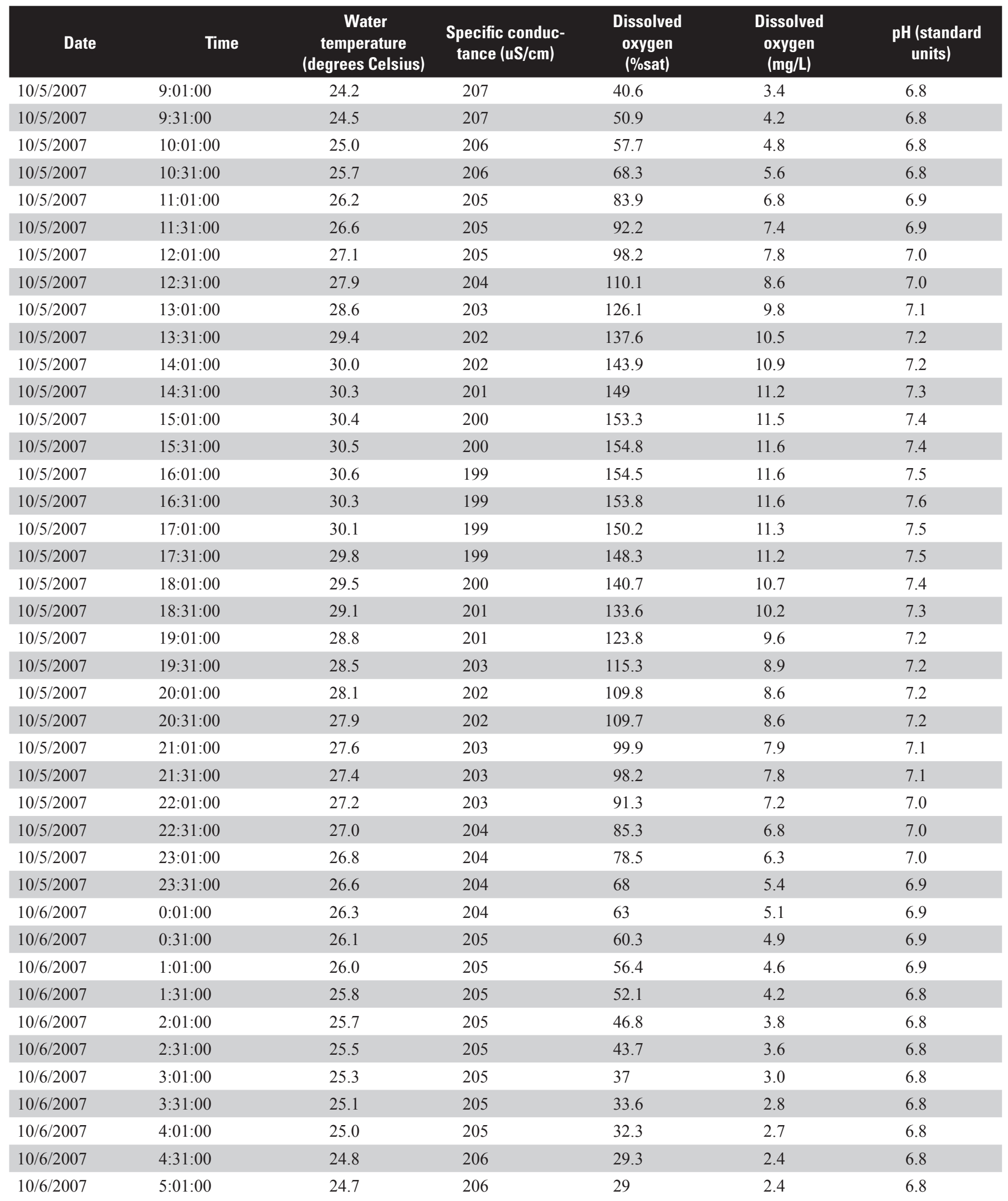


Table 6. Physical property data recorded at 30-minute intervals for station 07279970 Bobo Bayou at Bobo, Mississippi, October 4-9, 2007.-Continued

$[\mu \mathrm{S} / \mathrm{cm}$, microsiemens per centimeter; \%sat, percent saturation; mg/L, milligrams per liter]

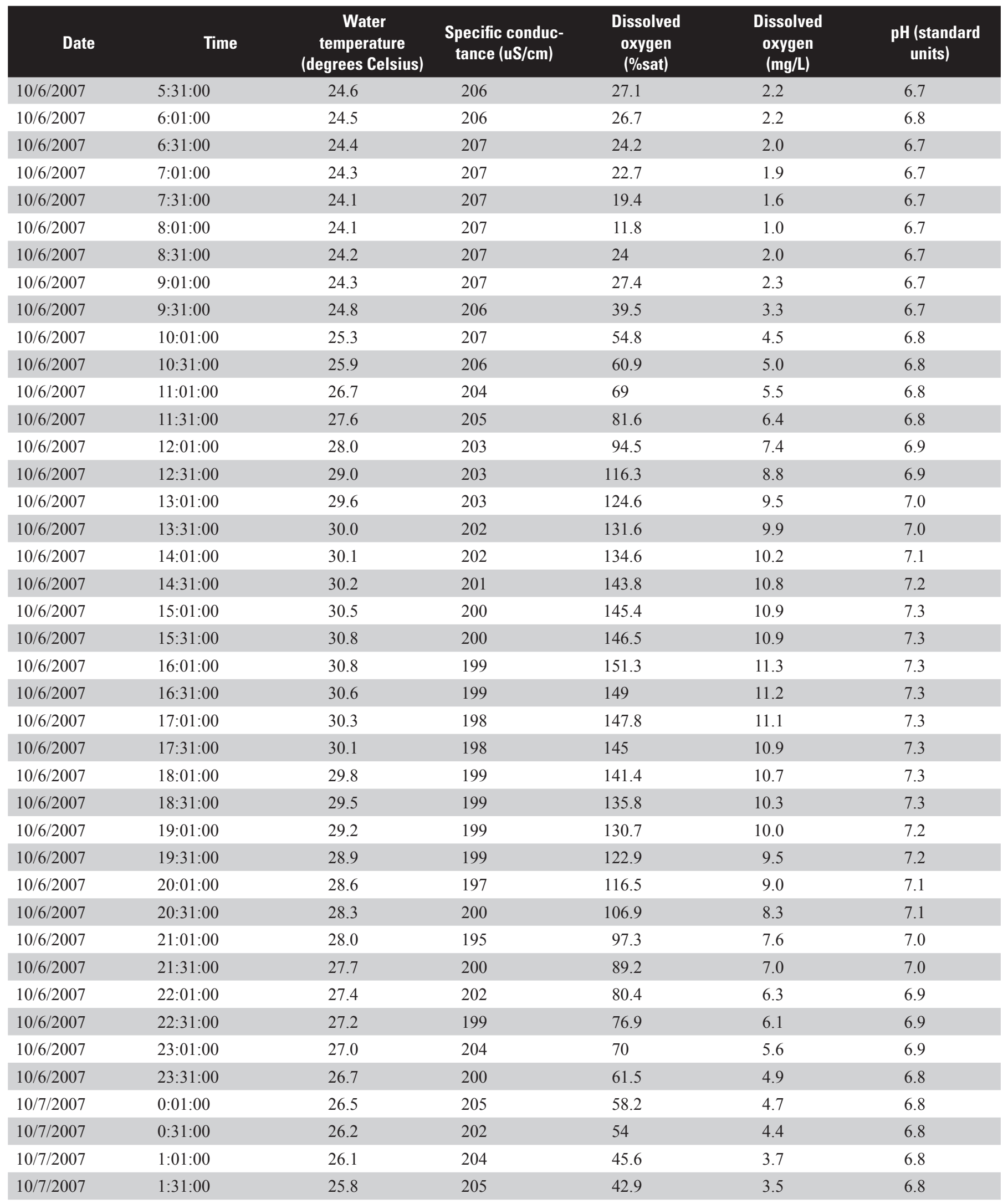


Table 6. Physical property data recorded at 30-minute intervals for station 07279970 Bobo Bayou at Bobo, Mississippi, October 4-9, 2007.-Continued

$[\mu \mathrm{S} / \mathrm{cm}$, microsiemens per centimeter; \%sat, percent saturation; $\mathrm{mg} / \mathrm{L}$, milligrams per liter $]$

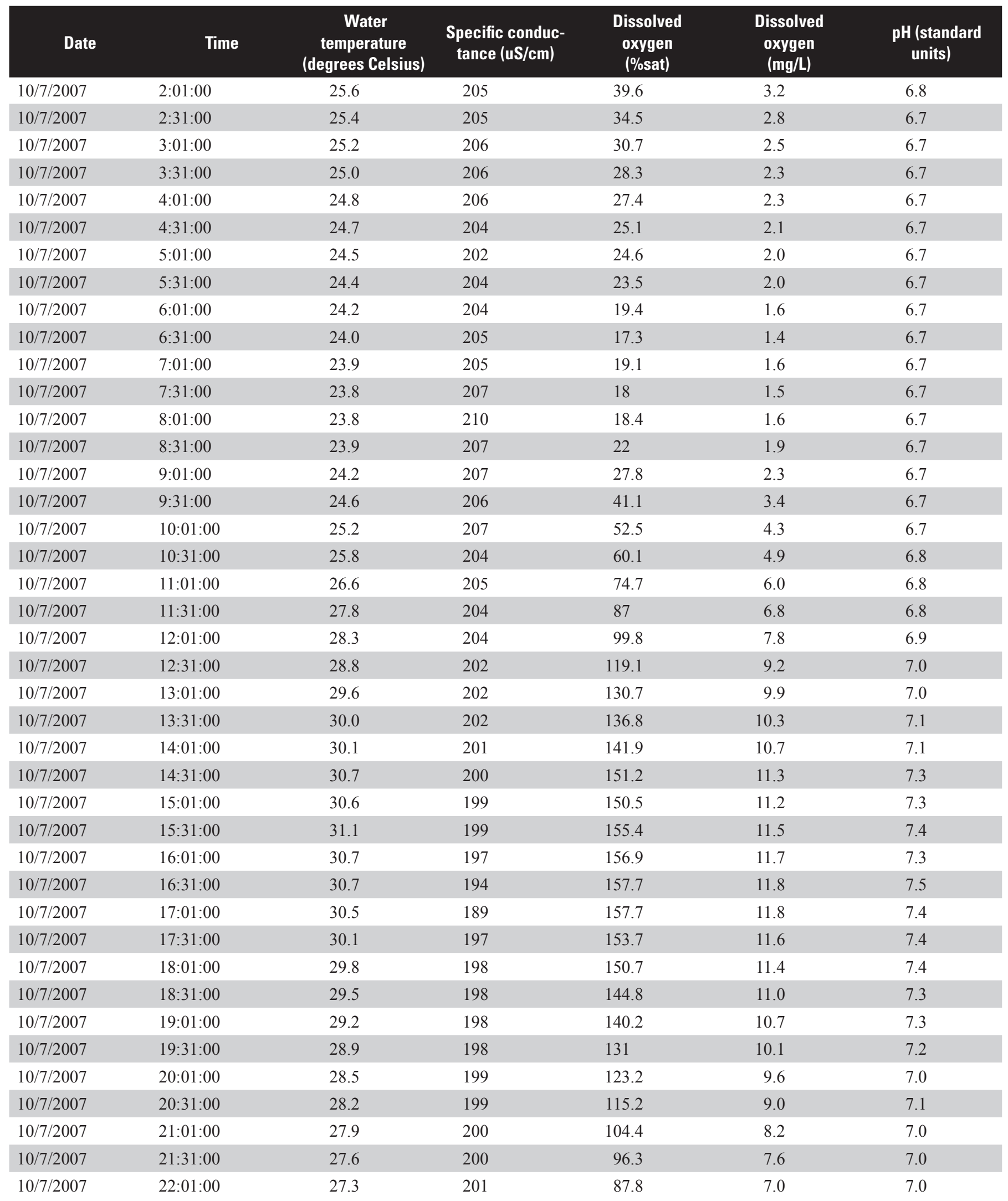


Table 6. Physical property data recorded at 30-minute intervals for station 07279970 Bobo Bayou at Bobo, Mississippi, October 4-9, 2007.-Continued

$[\mu \mathrm{S} / \mathrm{cm}$, microsiemens per centimeter; \%sat, percent saturation; mg/L, milligrams per liter]

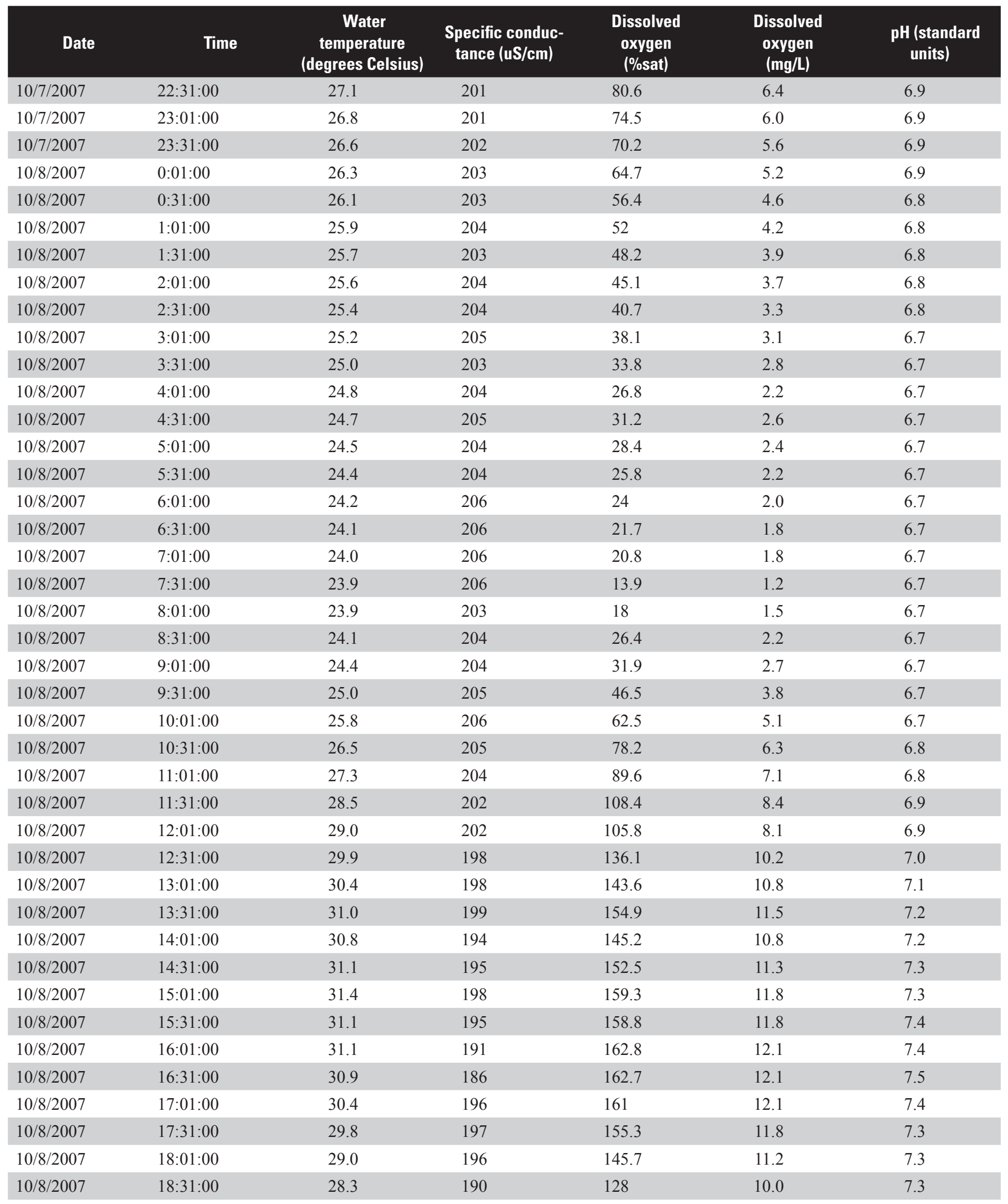


Table 6. Physical property data recorded at 30-minute intervals for station 07279970 Bobo Bayou at Bobo, Mississippi, October 4-9, 2007.-Continued

$[\mu \mathrm{S} / \mathrm{cm}$, microsiemens per centimeter; \%sat, percent saturation; $\mathrm{mg} / \mathrm{L}$, milligrams per liter]

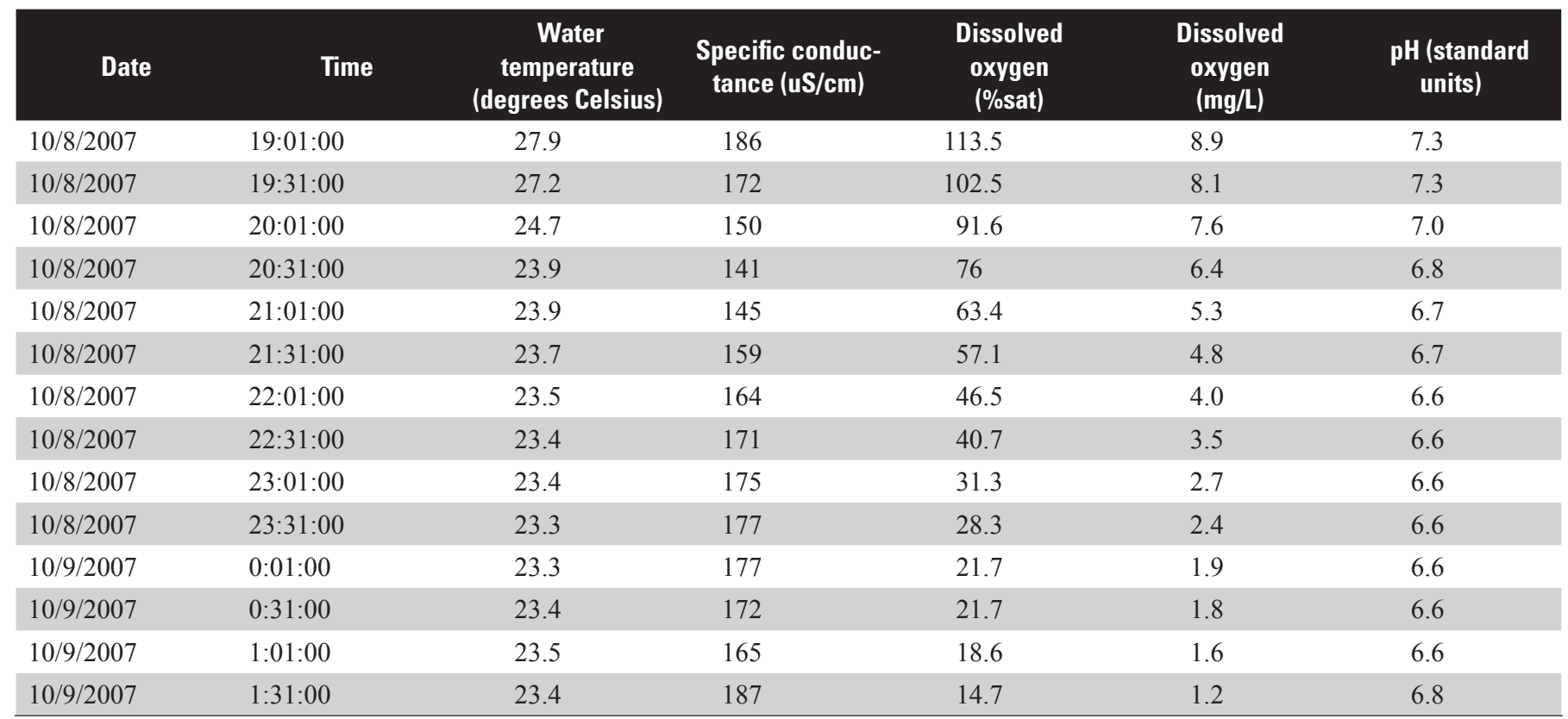


Table 7. Physical property data recorded at 30 -minute intervals for station 07280555 Tillatoba Creek west of Charleston, Mississippi, September 24-27, 2007.

$[\mu \mathrm{S} / \mathrm{cm}$, microsiemens per centimeter; \%sat, percent saturation; mg/L, milligrams per liter; FNU, formazin nephelometric units]

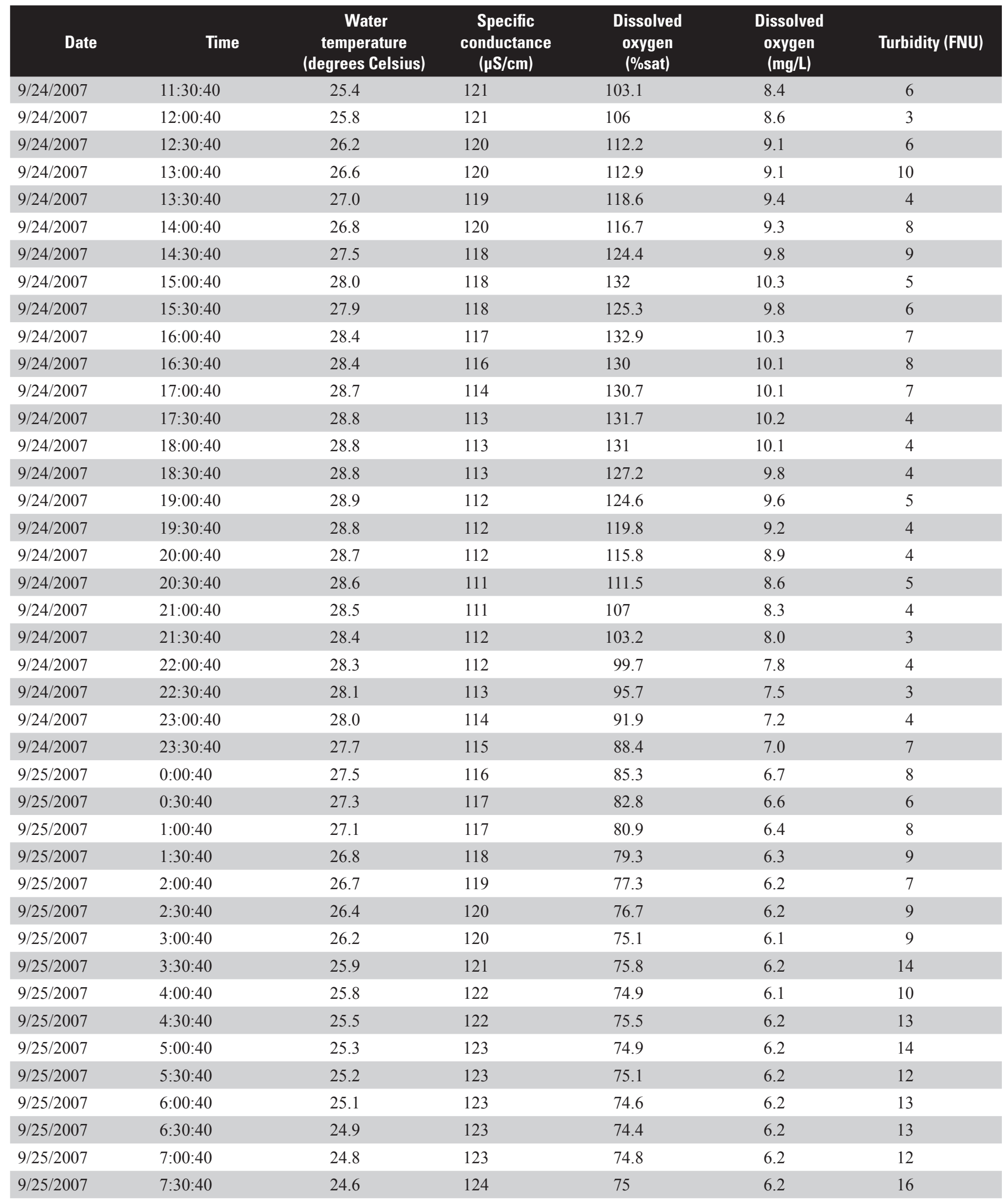


Table 7. Physical property data recorded at 30-minute intervals for station 07280555 Tillatoba Creek west of Charleston, Mississippi, September 24-27, 2007.-Continued

$[\mu \mathrm{S} / \mathrm{cm}$, microsiemens per centimeter; \%sat, percent saturation; mg/L, milligrams per liter; FNU, formazin nephelometric units]

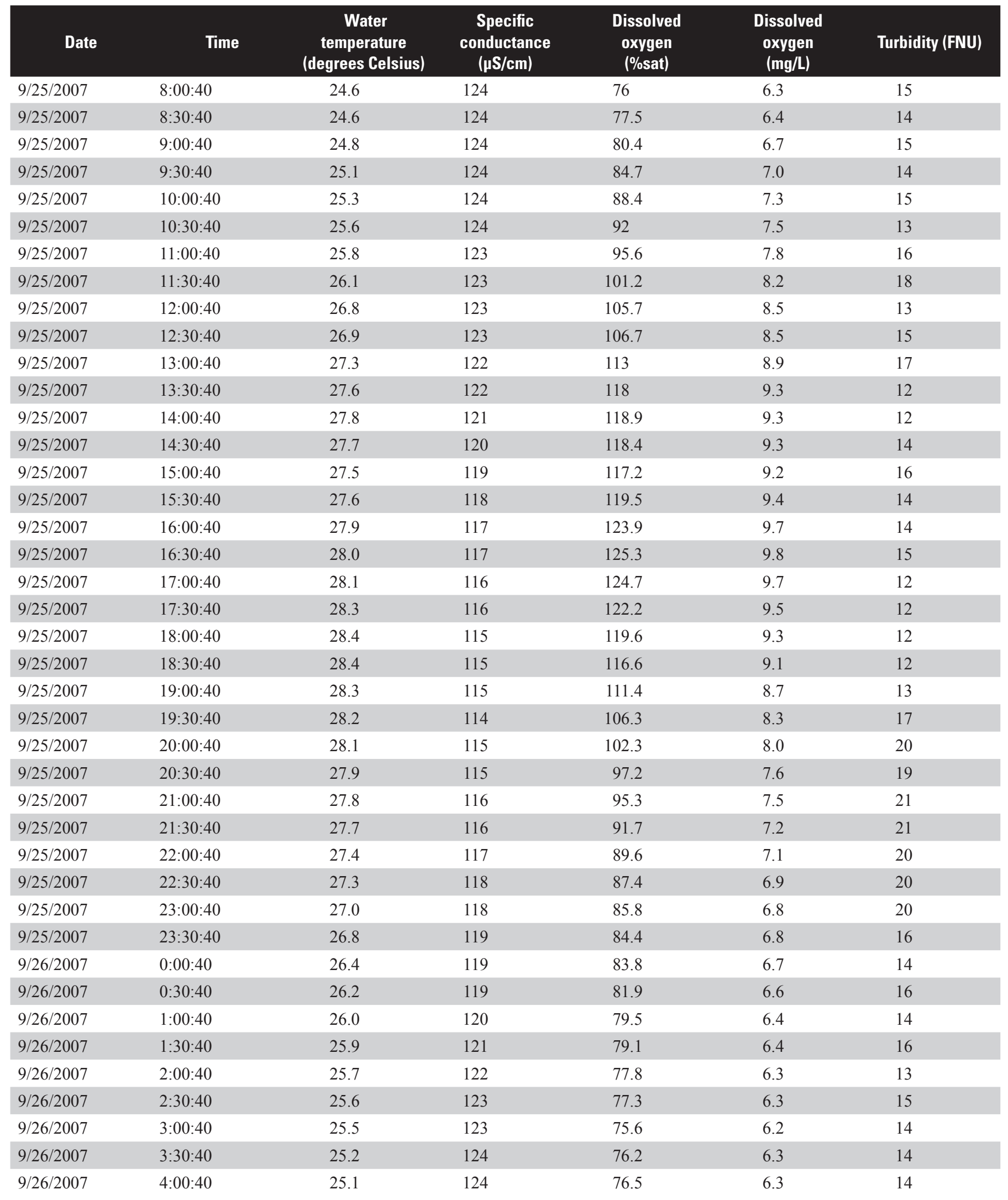


Table 7. Physical property data recorded at 30-minute intervals for station 07280555 Tillatoba Creek west of Charleston, Mississippi, September 24-27, 2007.-Continued

$[\mu \mathrm{S} / \mathrm{cm}$, microsiemens per centimeter; \%sat, percent saturation; mg/L, milligrams per liter; FNU, formazin nephelometric units]

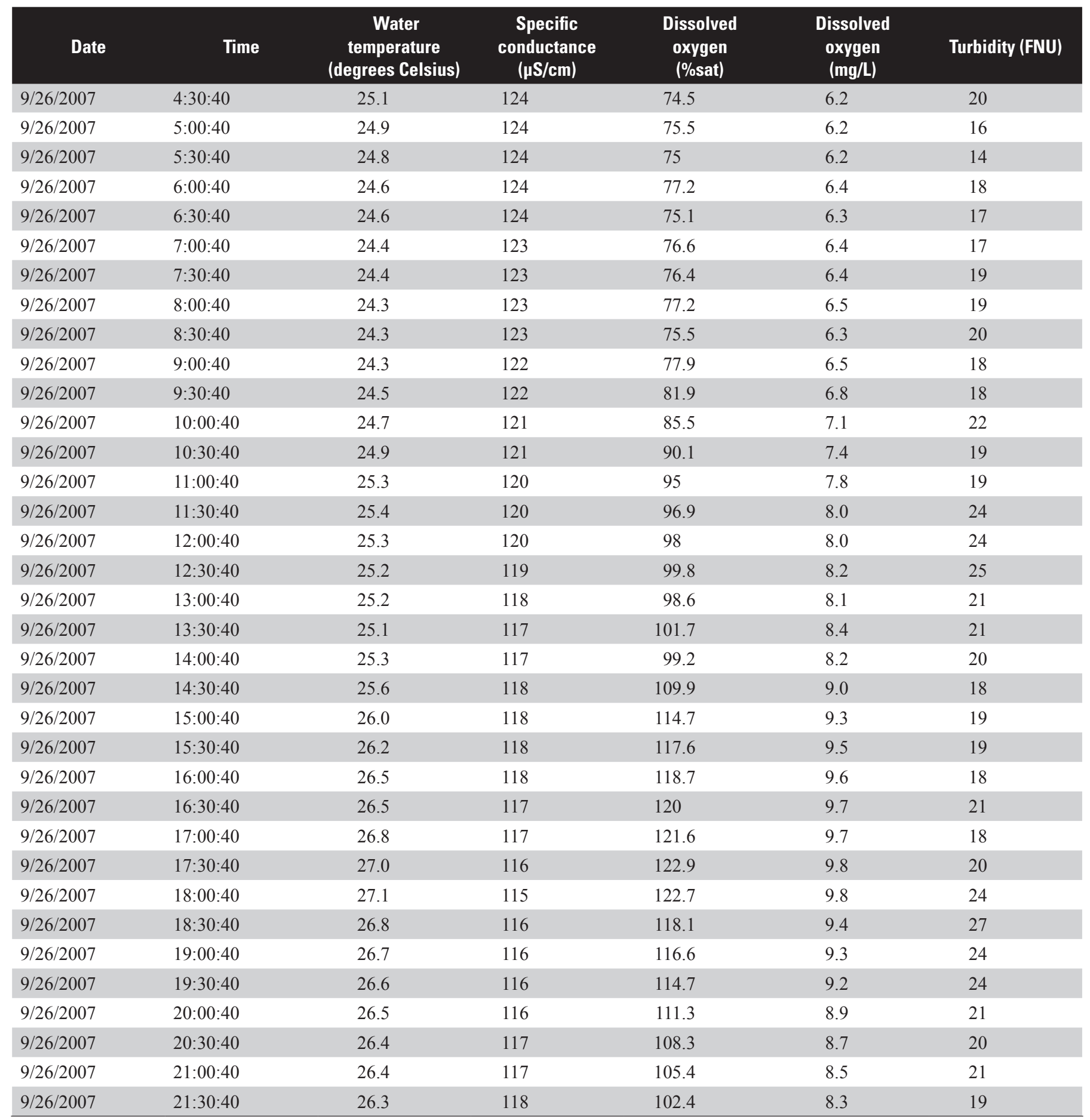


Table 8. Physical property data recorded at 30-minute intervals for station 07286006 Ascalmore Creek near Macel, Mississippi, October 3-9, 2007.

$[\mu \mathrm{S} / \mathrm{cm}$, microsiemens per centimeter; \%sat, percent saturation; $\mathrm{mg} / \mathrm{L}$, milligrams per liter]

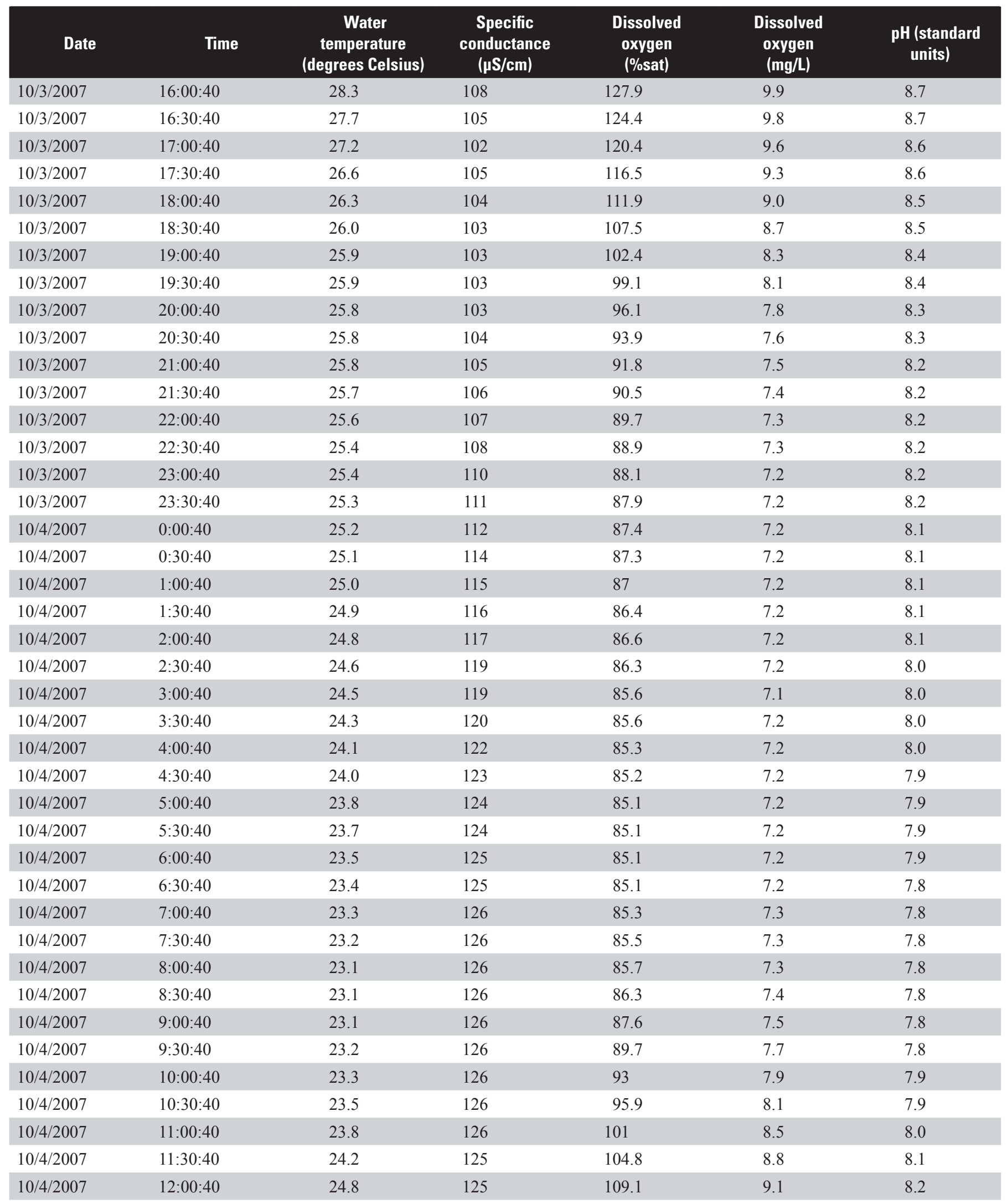


Table 8. Physical property data recorded at 30-minute intervals for station 07286006 Ascalmore Creek near Macel, Mississippi, October 3-9, 2007.-Continued

$[\mu \mathrm{S} / \mathrm{cm}$, microsiemens per centimeter; \%sat, percent saturation; $\mathrm{mg} / \mathrm{L}$, milligrams per liter]

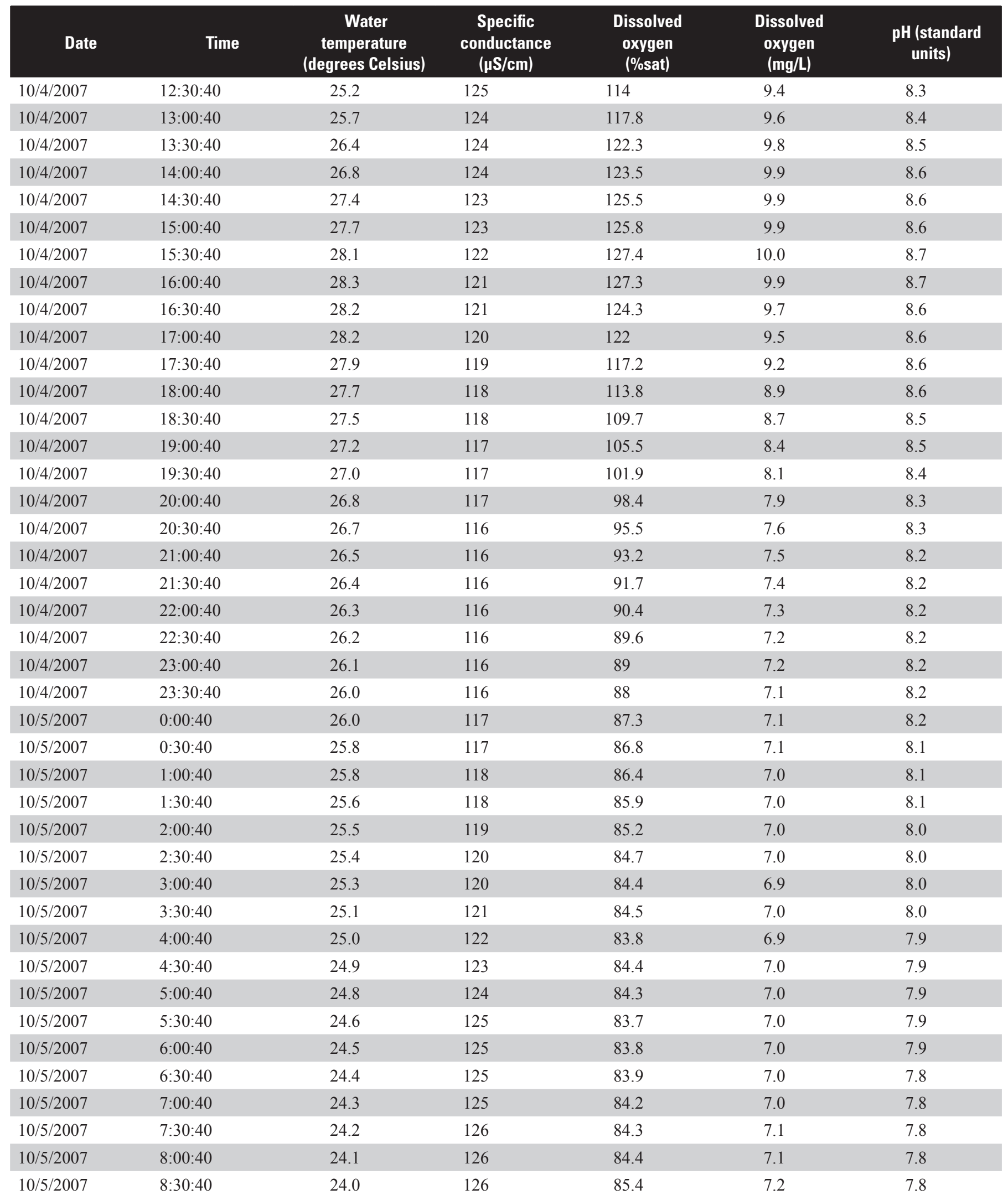


Table 8. Physical property data recorded at 30-minute intervals for station 07286006 Ascalmore Creek near Macel, Mississippi, October 3-9, 2007.-Continued

$[\mu \mathrm{S} / \mathrm{cm}$, microsiemens per centimeter; \%sat, percent saturation; $\mathrm{mg} / \mathrm{L}$, milligrams per liter $]$

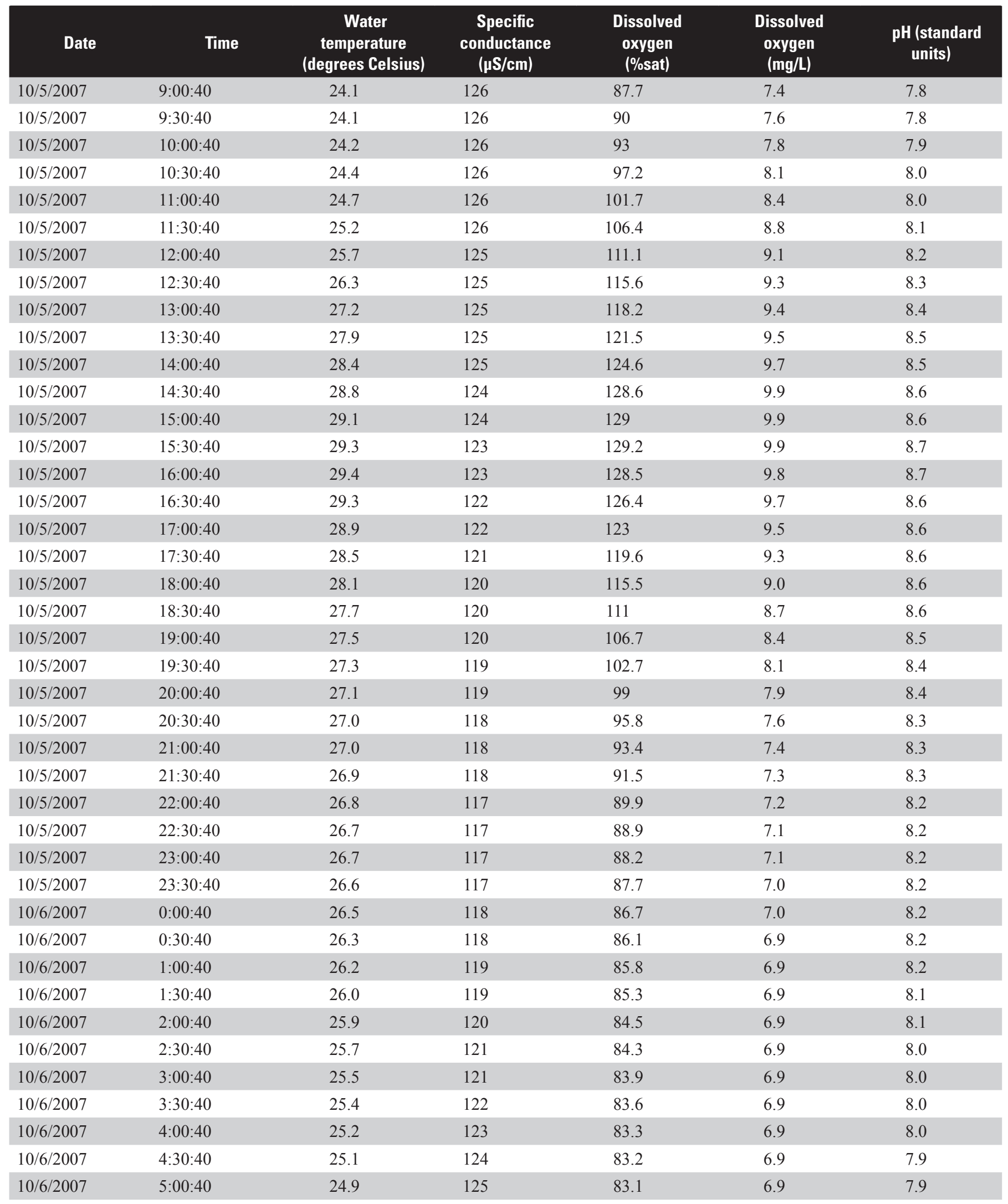


Table 8. Physical property data recorded at 30-minute intervals for station 07286006 Ascalmore Creek near Macel, Mississippi, October 3-9, 2007.-Continued

$[\mu \mathrm{S} / \mathrm{cm}$, microsiemens per centimeter; \%sat, percent saturation; mg/L, milligrams per liter]

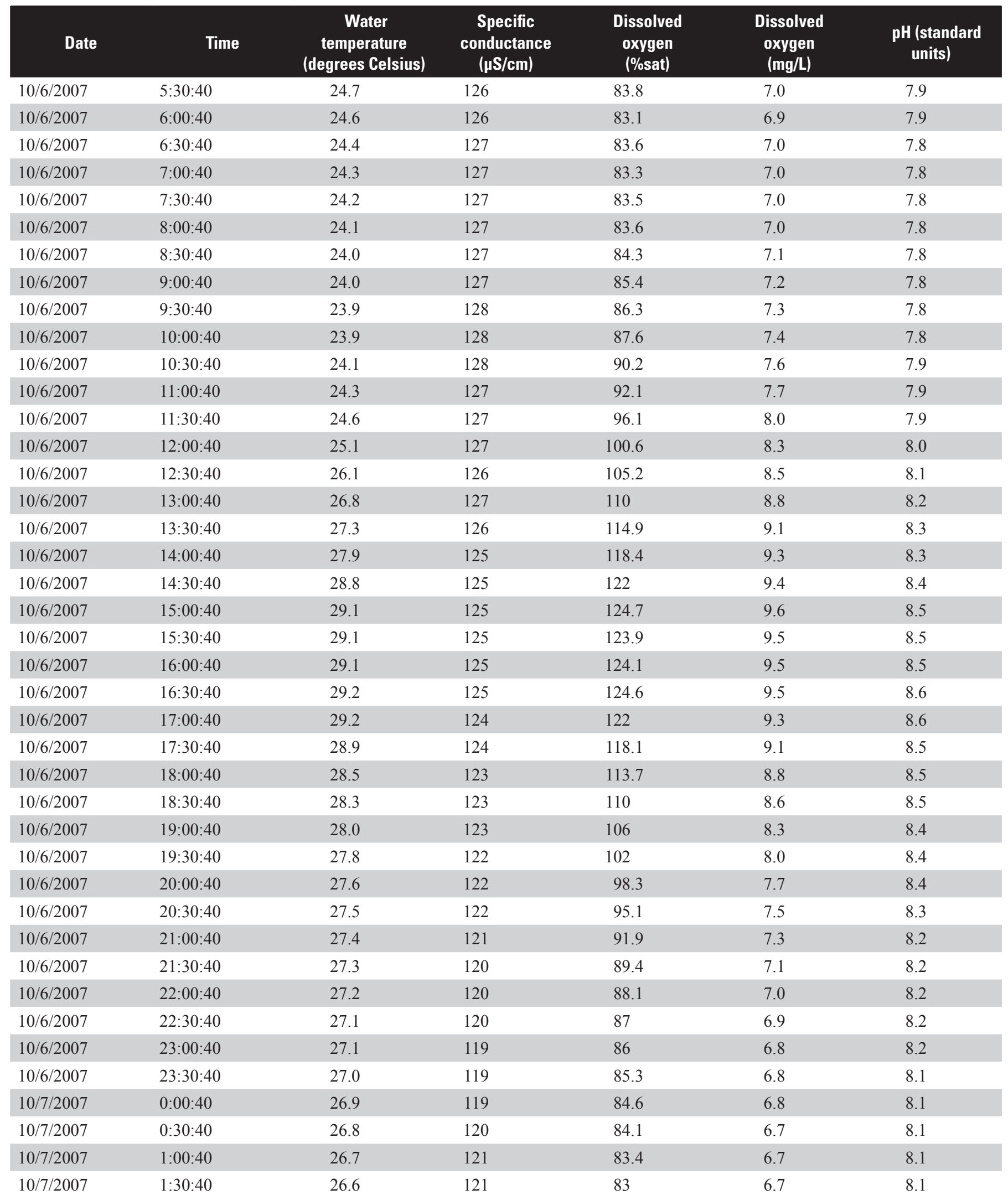


Table 8. Physical property data recorded at 30-minute intervals for station 07286006 Ascalmore Creek near Macel, Mississippi, October 3-9, 2007.-Continued

$[\mu \mathrm{S} / \mathrm{cm}$, microsiemens per centimeter; \%sat, percent saturation; $\mathrm{mg} / \mathrm{L}$, milligrams per liter $]$

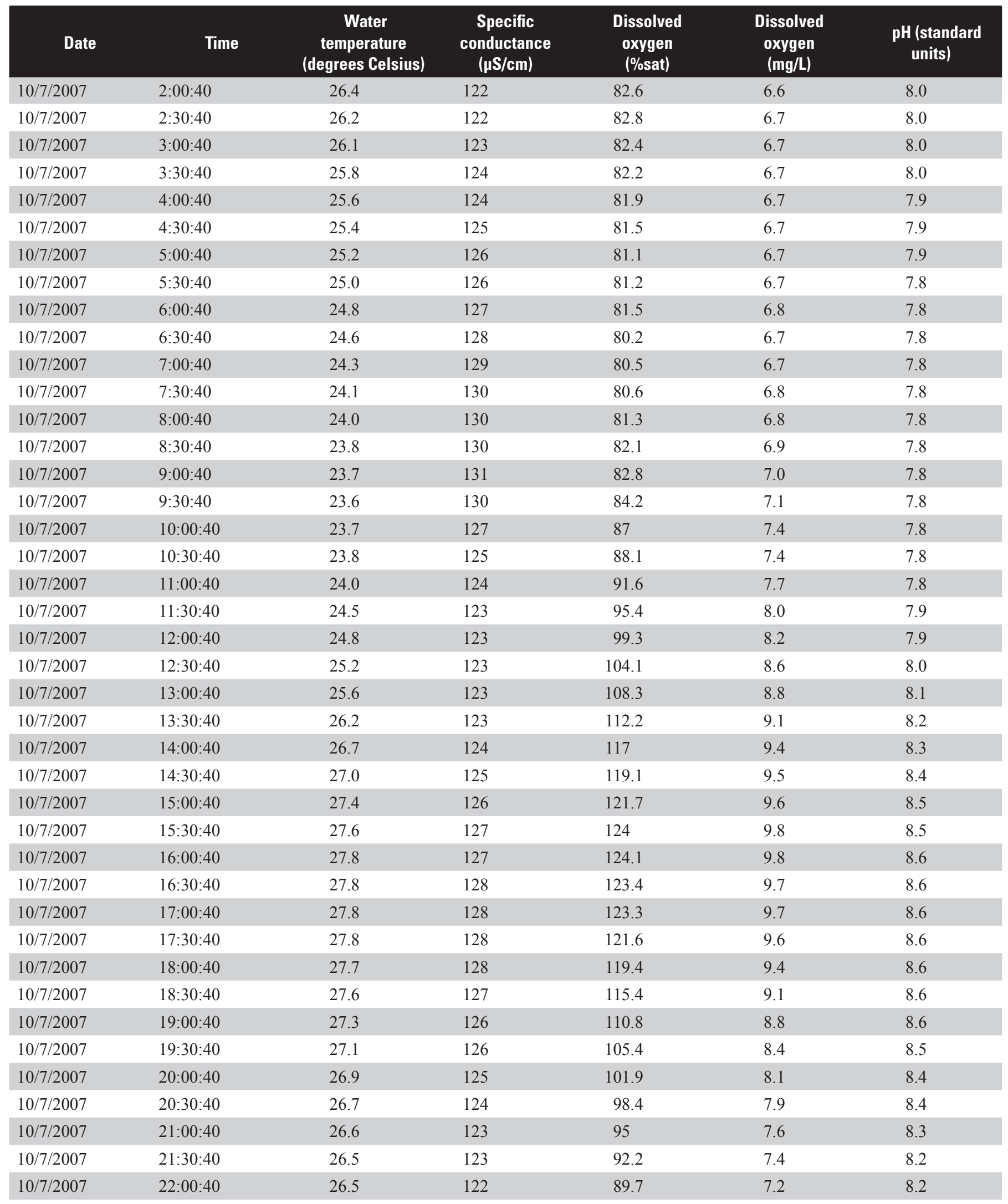


Table 8. Physical property data recorded at 30-minute intervals for station 07286006 Ascalmore Creek near Macel, Mississippi, October 3-9, 2007.-Continued

$[\mu \mathrm{S} / \mathrm{cm}$, microsiemens per centimeter; \%sat, percent saturation; $\mathrm{mg} / \mathrm{L}$, milligrams per liter]

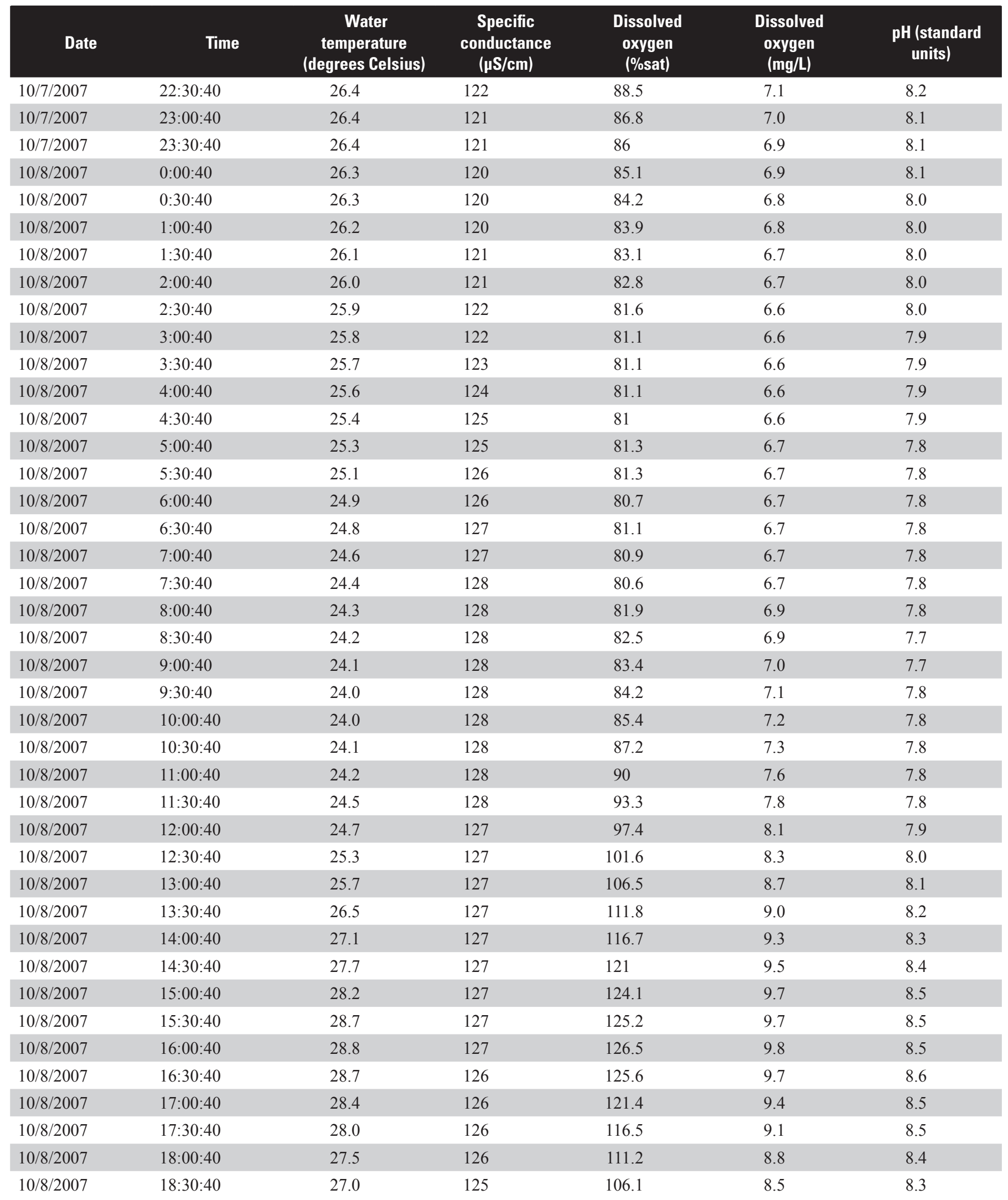


Table 8. Physical property data recorded at 30-minute intervals for station 07286006 Ascalmore Creek near Macel, Mississippi, October 3-9, 2007.-Continued

$[\mu \mathrm{S} / \mathrm{cm}$, microsiemens per centimeter; \%sat, percent saturation; $\mathrm{mg} / \mathrm{L}$, milligrams per liter]

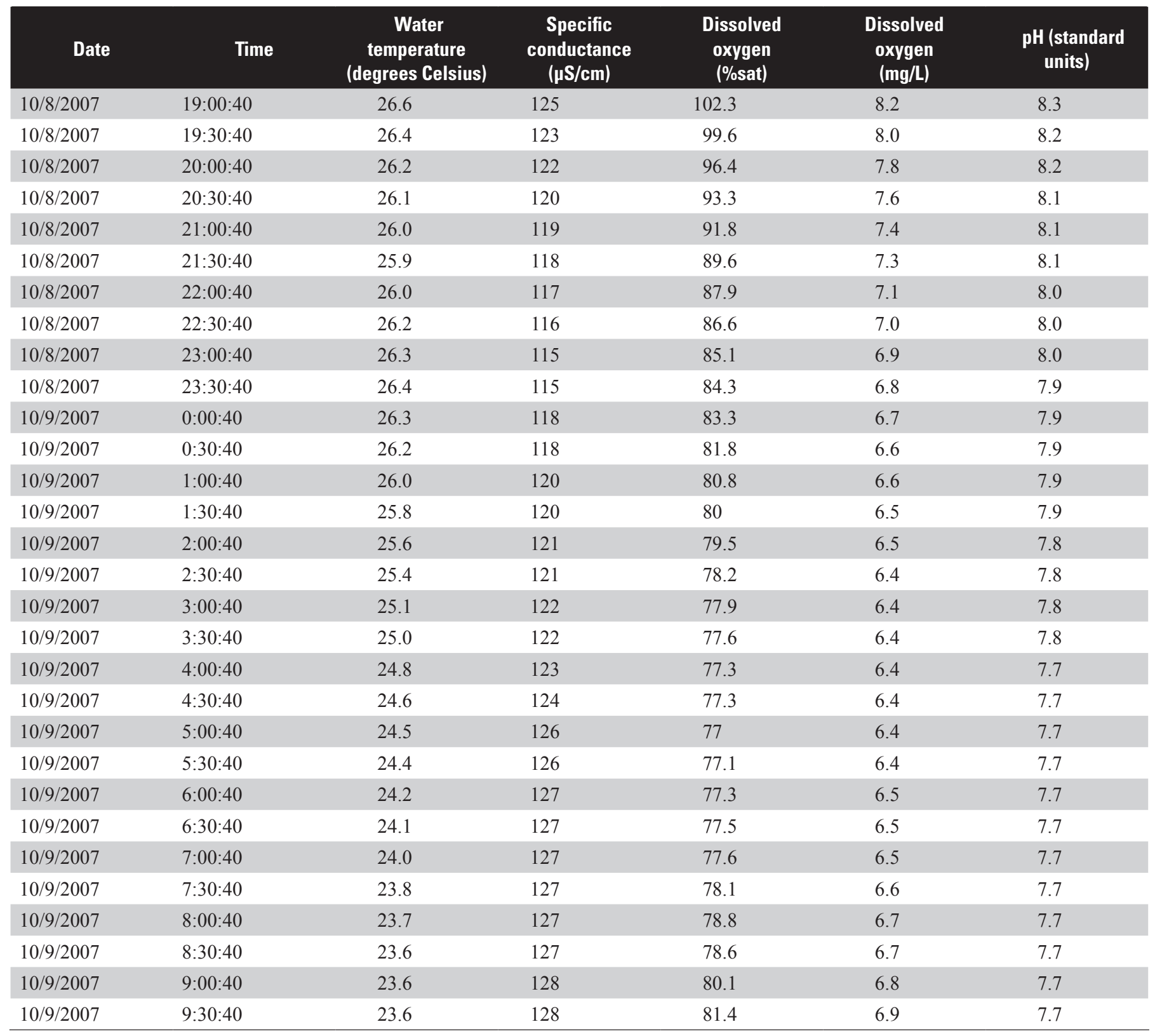


Table 9. Physical property data recorded at 30-minute intervals for station 07286011 Brushy Creek near Macel, Mississippi, October 1-3, 2007.

$[\mu \mathrm{S} / \mathrm{cm}$, microsiemens per centimeter; \%sat, percent saturation; $\mathrm{mg} / \mathrm{L}$, milligrams per liter]

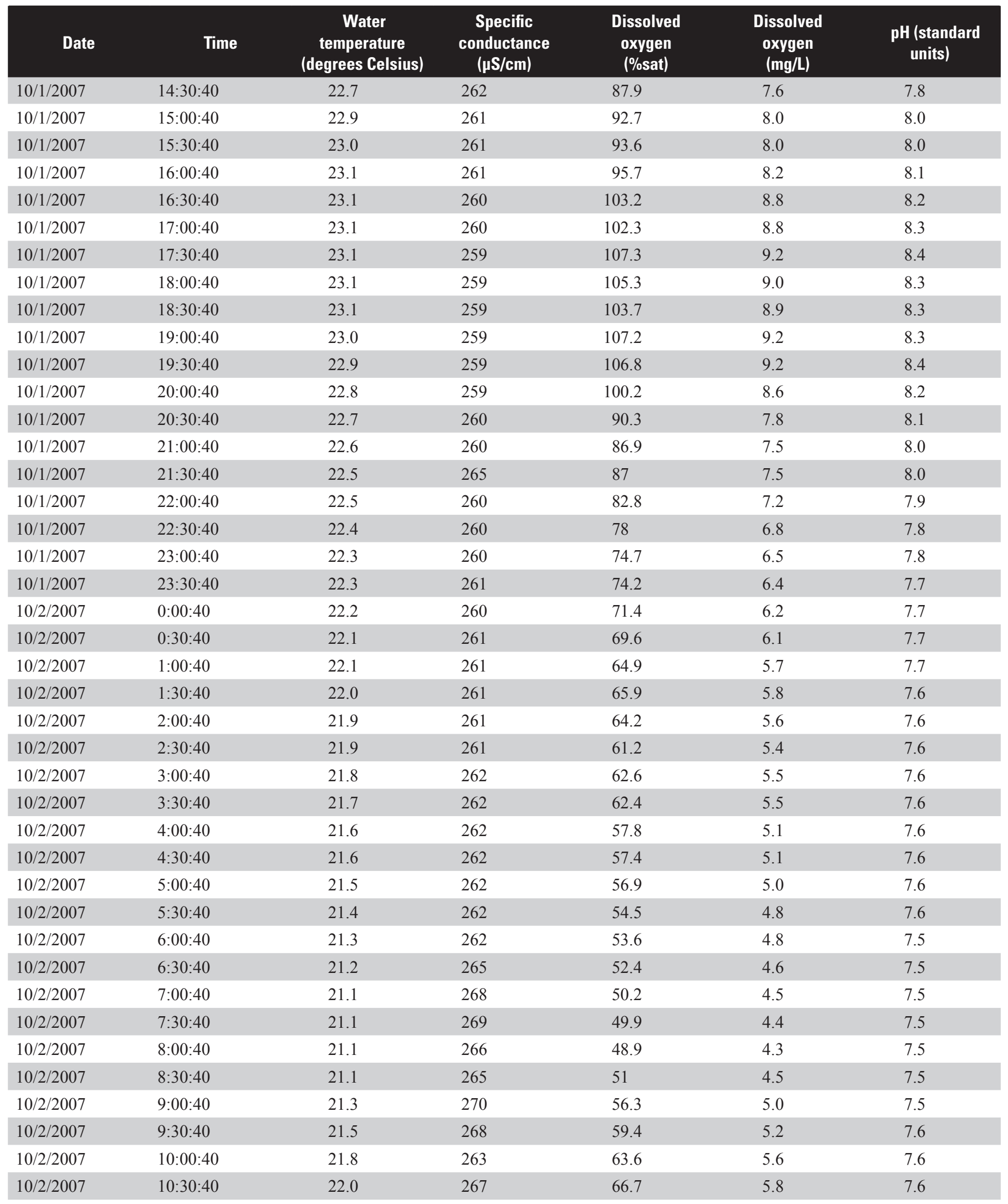


Table 9. Physical property data recorded at 30-minute intervals for station 07286011 Brushy Creek near Macel, Mississippi, October 1-3, 2007.-Continued

$[\mu \mathrm{S} / \mathrm{cm}$, microsiemens per centimeter; \%sat, percent saturation; $\mathrm{mg} / \mathrm{L}$, milligrams per liter $]$

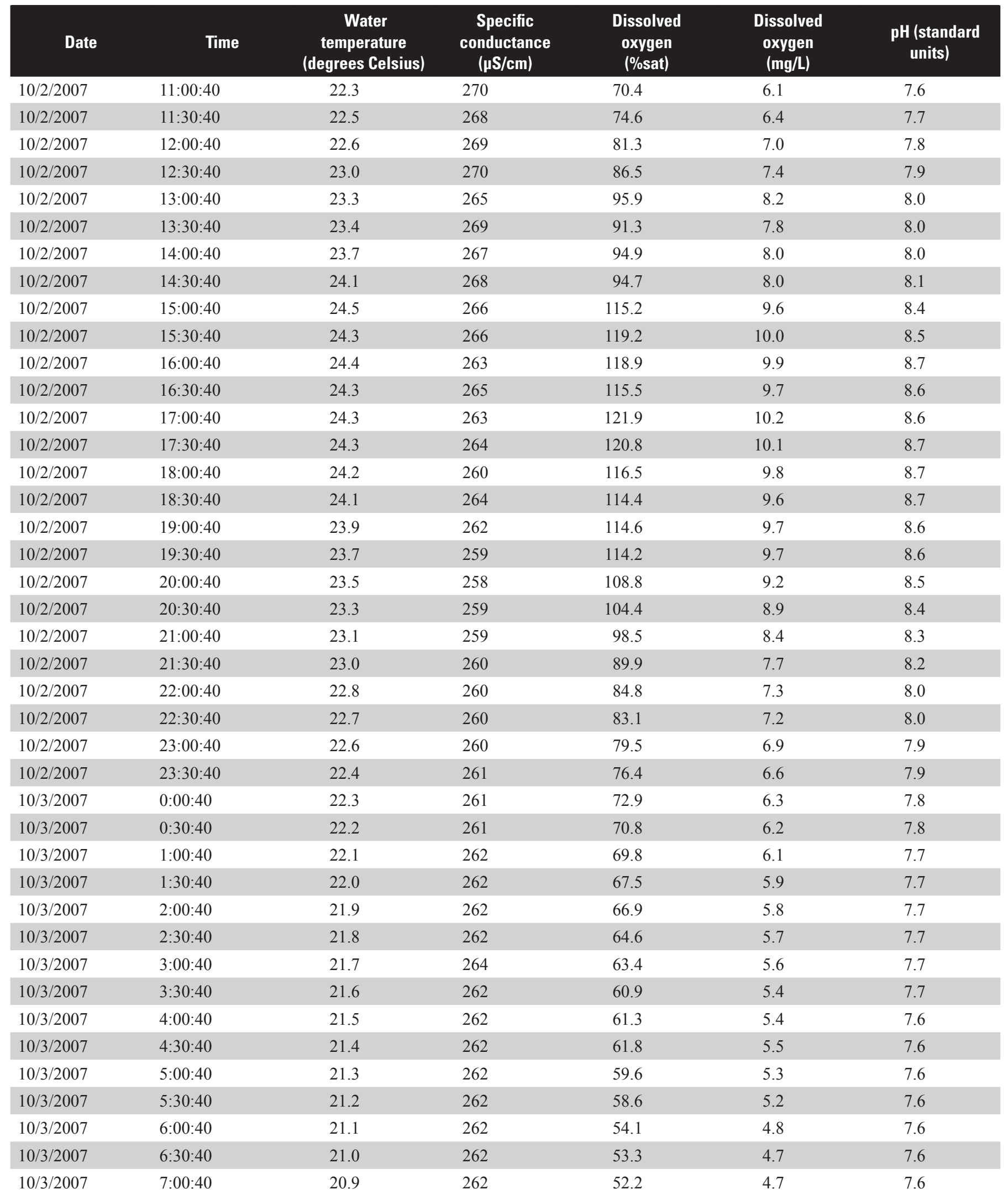


Table 9. Physical property data recorded at 30-minute intervals for station 07286011 Brushy Creek near Macel, Mississippi, October 1-3, 2007.-Continued

$[\mu \mathrm{S} / \mathrm{cm}$, microsiemens per centimeter; \%sat, percent saturation; mg/L, milligrams per liter]

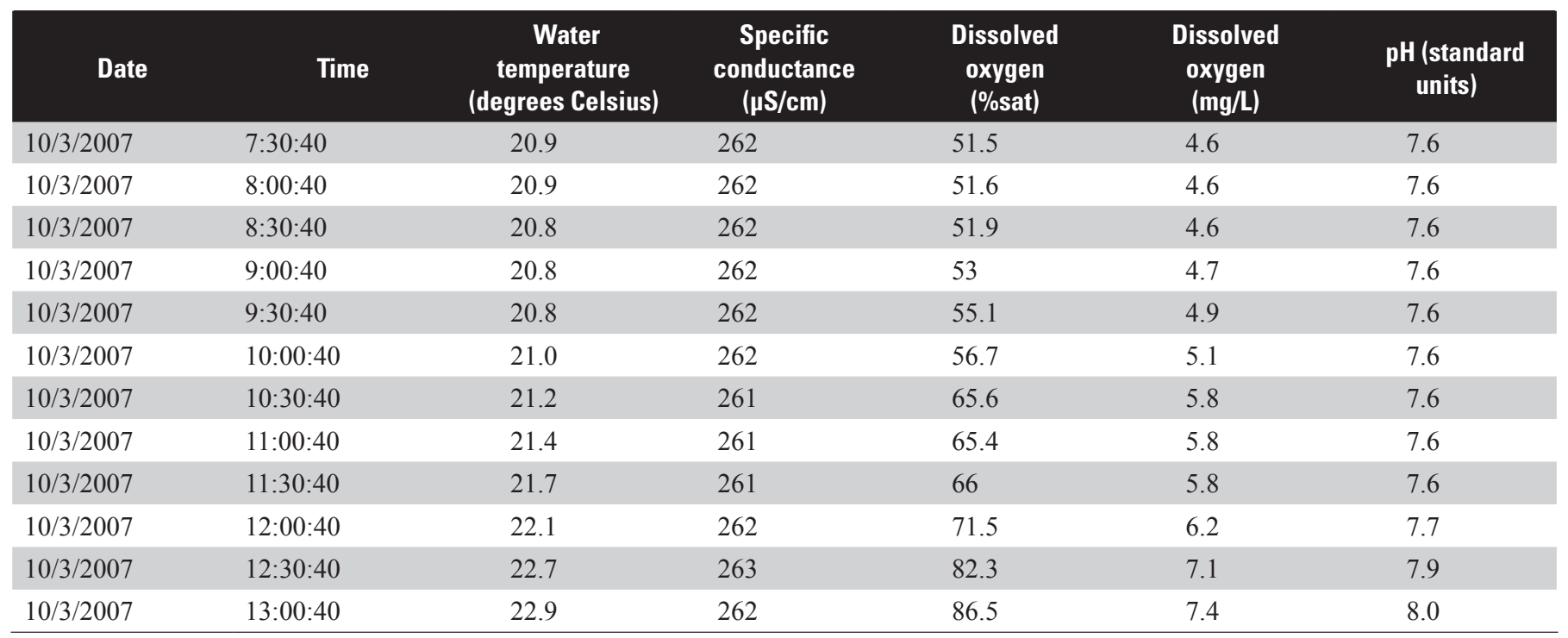


Table 10. Physical property data recorded at 30-minute intervals for station 07287310 Spring Branch near Tchula, Mississippi, September 11-13, 2007.

$[\mu \mathrm{S} / \mathrm{cm}$, microsiemens per centimeter; \%sat, percent saturation; mg/L, milligrams per liter; FNU, formazin nephelometric units]

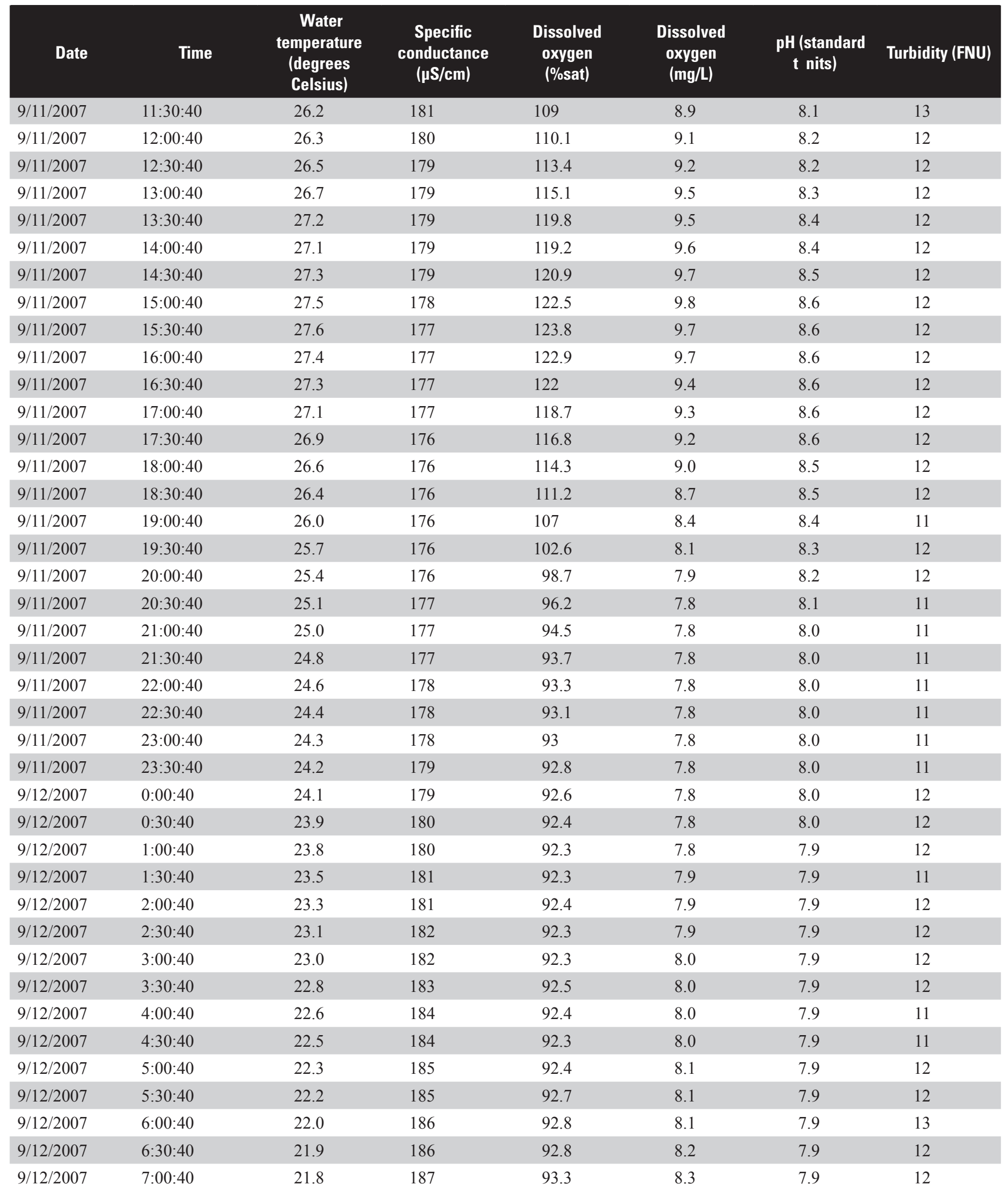


Table 10. Physical property data recorded at 30-minute intervals for station 07287310 Spring Branch near Tchula, Mississippi, September 11-13, 2007.-Continued

$[\mu \mathrm{S} / \mathrm{cm}$, microsiemens per centimeter; \%sat, percent saturation; mg/L, milligrams per liter; FNU, formazin nephelometric units $]$

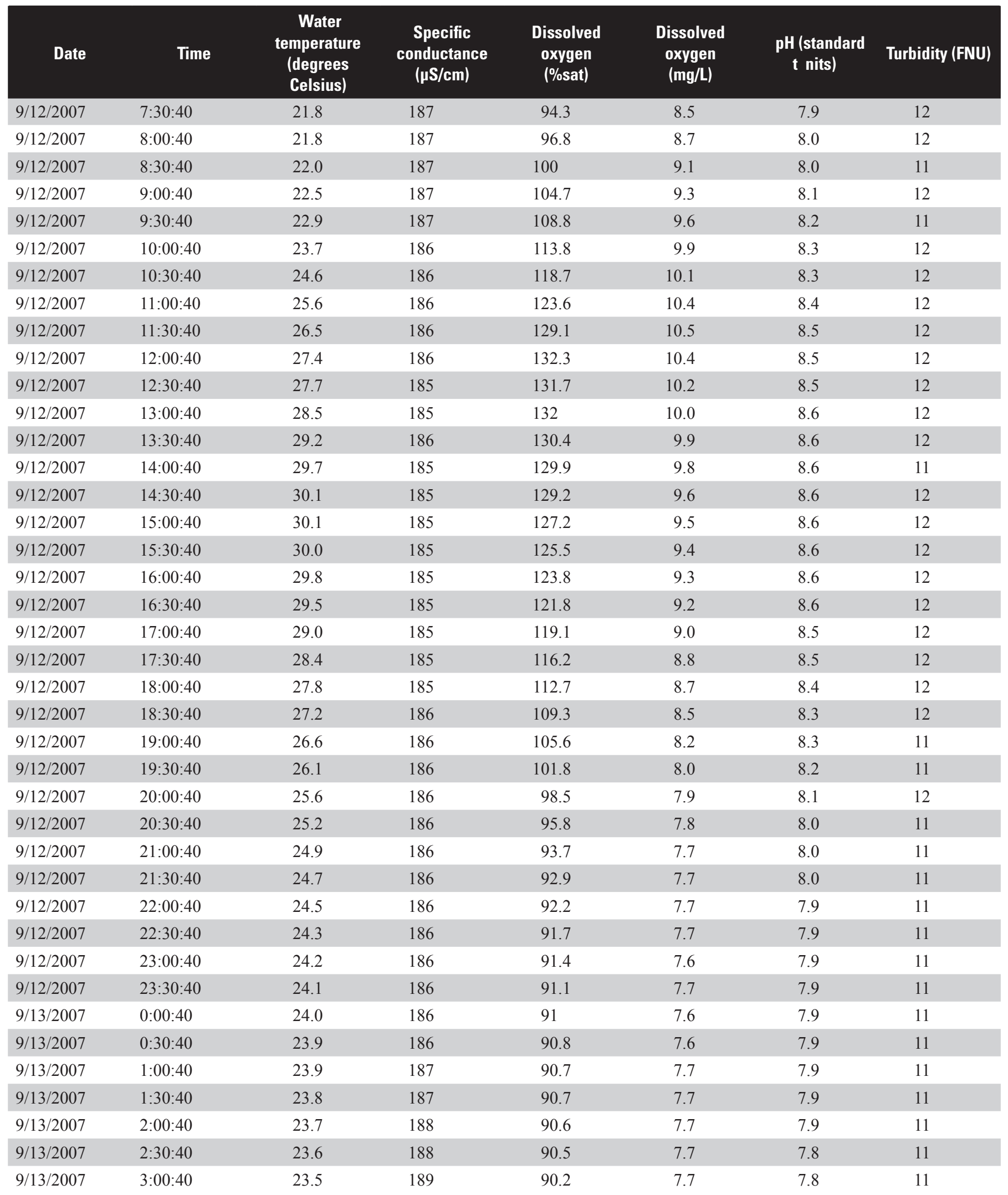


Table 10. Physical property data recorded at 30-minute intervals for station 07287310 Spring Branch near Tchula, Mississippi, September 11-13, 2007.-Continued

$[\mu \mathrm{S} / \mathrm{cm}$, microsiemens per centimeter; \%sat, percent saturation; mg/L, milligrams per liter; FNU, formazin nephelometric units]

\begin{tabular}{|c|c|c|c|c|c|c|c|}
\hline Date & Time & $\begin{array}{c}\text { Water } \\
\text { temperature } \\
\text { (degrees } \\
\text { Celsius) }\end{array}$ & $\begin{array}{c}\text { Specific } \\
\text { conductance } \\
\text { (pS/cm) }\end{array}$ & $\begin{array}{c}\text { Dissolved } \\
\text { oxygen } \\
\text { (\%sat) }\end{array}$ & $\begin{array}{c}\text { Dissolved } \\
\text { oxygen } \\
\text { (mg/L) }\end{array}$ & $\begin{array}{c}\text { pH (standard } \\
\text { t nits) }\end{array}$ & Turbidity (FNU) \\
\hline $9 / 13 / 2007$ & 4:00:40 & 23.4 & 190 & 90.2 & 7.7 & 7.8 & 11 \\
\hline $9 / 13 / 2007$ & $4: 30: 40$ & 23.3 & 190 & 90.6 & 7.8 & 7.8 & 11 \\
\hline $9 / 13 / 2007$ & $5: 30: 40$ & 23.3 & 192 & 91 & 7.8 & 7.8 & 11 \\
\hline $9 / 13 / 2007$ & $6: 00: 40$ & 23.3 & 192 & 91.2 & 7.8 & 7.8 & 11 \\
\hline $9 / 13 / 2007$ & $6: 30: 40$ & 23.2 & 193 & 91.4 & 7.8 & 7.8 & 11 \\
\hline $9 / 13 / 2007$ & 7:00:40 & 23.2 & 194 & 91.5 & 7.8 & 7.8 & 11 \\
\hline $9 / 13 / 2007$ & 9:00:40 & 23.6 & 194 & 97.7 & 8.6 & 7.9 & 11 \\
\hline $9 / 13 / 2007$ & $9: 30: 40$ & 23.8 & 194 & 101.3 & 8.8 & 8.0 & 11 \\
\hline $9 / 13 / 2007$ & 10:00:40 & 24.2 & 194 & 105.3 & 9.2 & 8.1 & 11 \\
\hline $9 / 13 / 2007$ & $10: 30: 40$ & 24.6 & 193 & 110 & 9.5 & 8.2 & 11 \\
\hline $9 / 13 / 2007$ & $11: 00: 40$ & 25.1 & 192 & 115.5 & 9.8 & 8.2 & 11 \\
\hline $9 / 13 / 2007$ & $11: 30: 40$ & 25.6 & 192 & 119.7 & 10.0 & 8.3 & 11 \\
\hline $9 / 13 / 2007$ & $12: 00: 40$ & 26.0 & 191 & 122.9 & 10.1 & 8.4 & 11 \\
\hline $9 / 13 / 2007$ & $12: 30: 40$ & 26.4 & 190 & 124.9 & 10.1 & 8.5 & 11 \\
\hline $9 / 13 / 2007$ & $13: 00: 40$ & 26.5 & 190 & 125.7 & 10.0 & 8.5 & 11 \\
\hline
\end{tabular}


Table 11. Physical property data recorded at 30-minute intervals for station 07288185 Black Bayou at Baltzer, Mississippi, October 4-9, 2007.

$[\mu \mathrm{S} / \mathrm{cm}$, microsiemens per centimeter; \%sat, percent saturation; mg/L, milligrams per liter; FNU, formazin nephelometric unit; -- , no data]

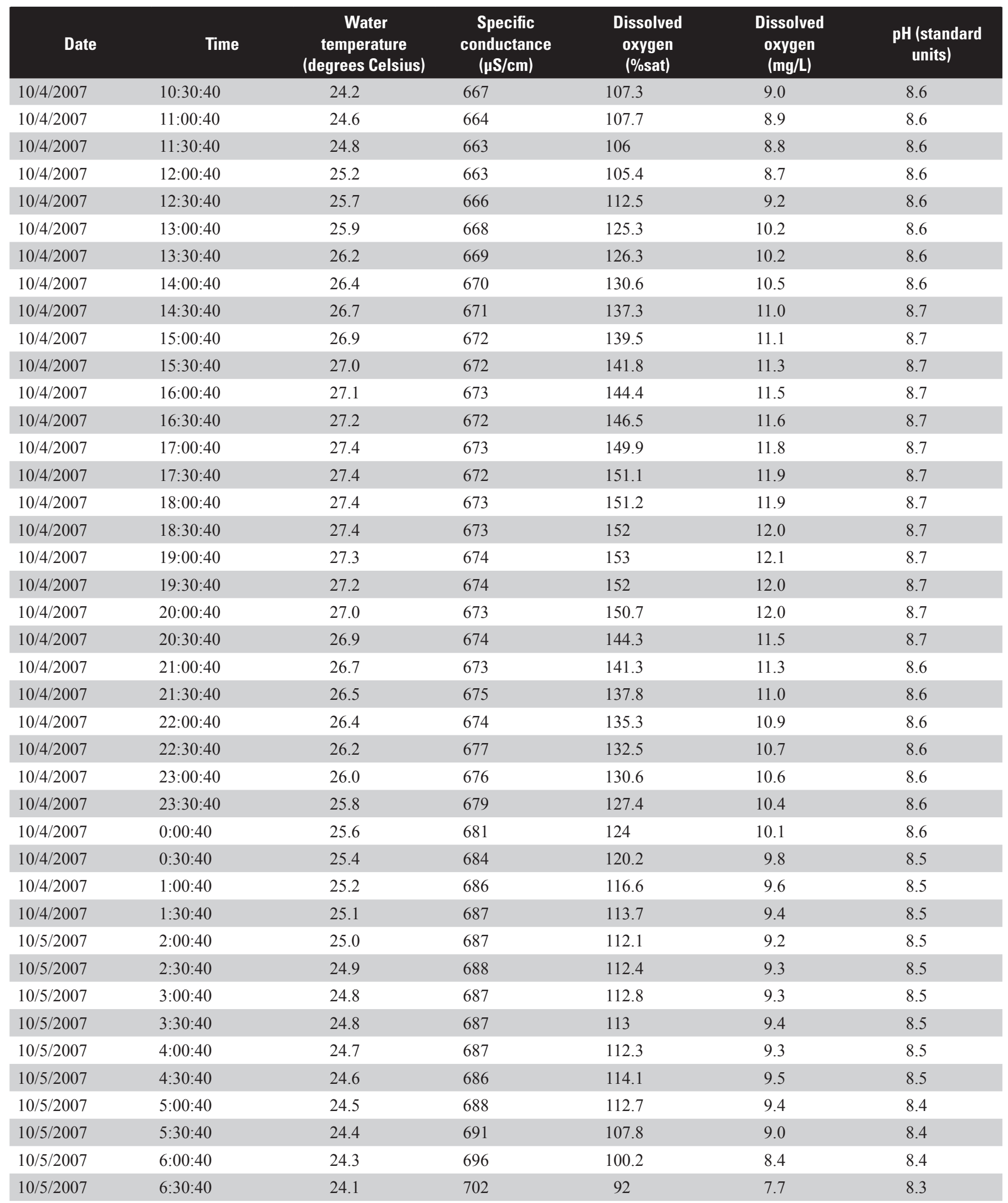


Table 11. Physical property data recorded at 30-minute intervals for station 07288185 Black Bayou at Baltzer, Mississippi, October 4-9, 2007.-Continued

$[\mu \mathrm{S} / \mathrm{cm}$, microsiemens per centimeter; \%sat, percent saturation; mg/L, milligrams per liter; FNU, formazin nephelometric unit; -- , no data]

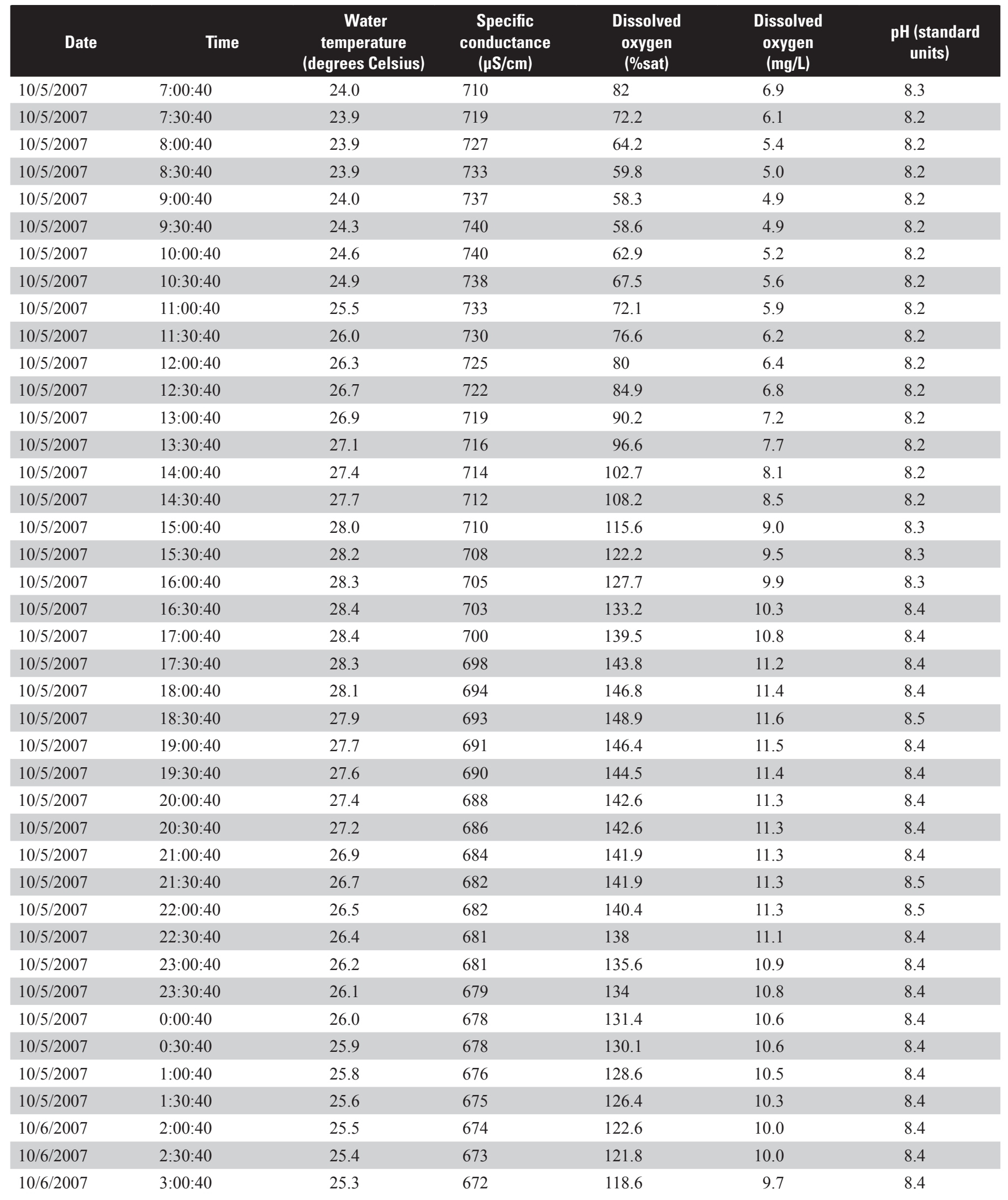


Table 11. Physical property data recorded at 30-minute intervals for station 07288185 Black Bayou at Baltzer, Mississippi, October 4-9, 2007.-Continued

$[\mu \mathrm{S} / \mathrm{cm}$, microsiemens per centimeter; \%sat, percent saturation; mg/L, milligrams per liter; FNU, formazin nephelometric unit; -- , no data]

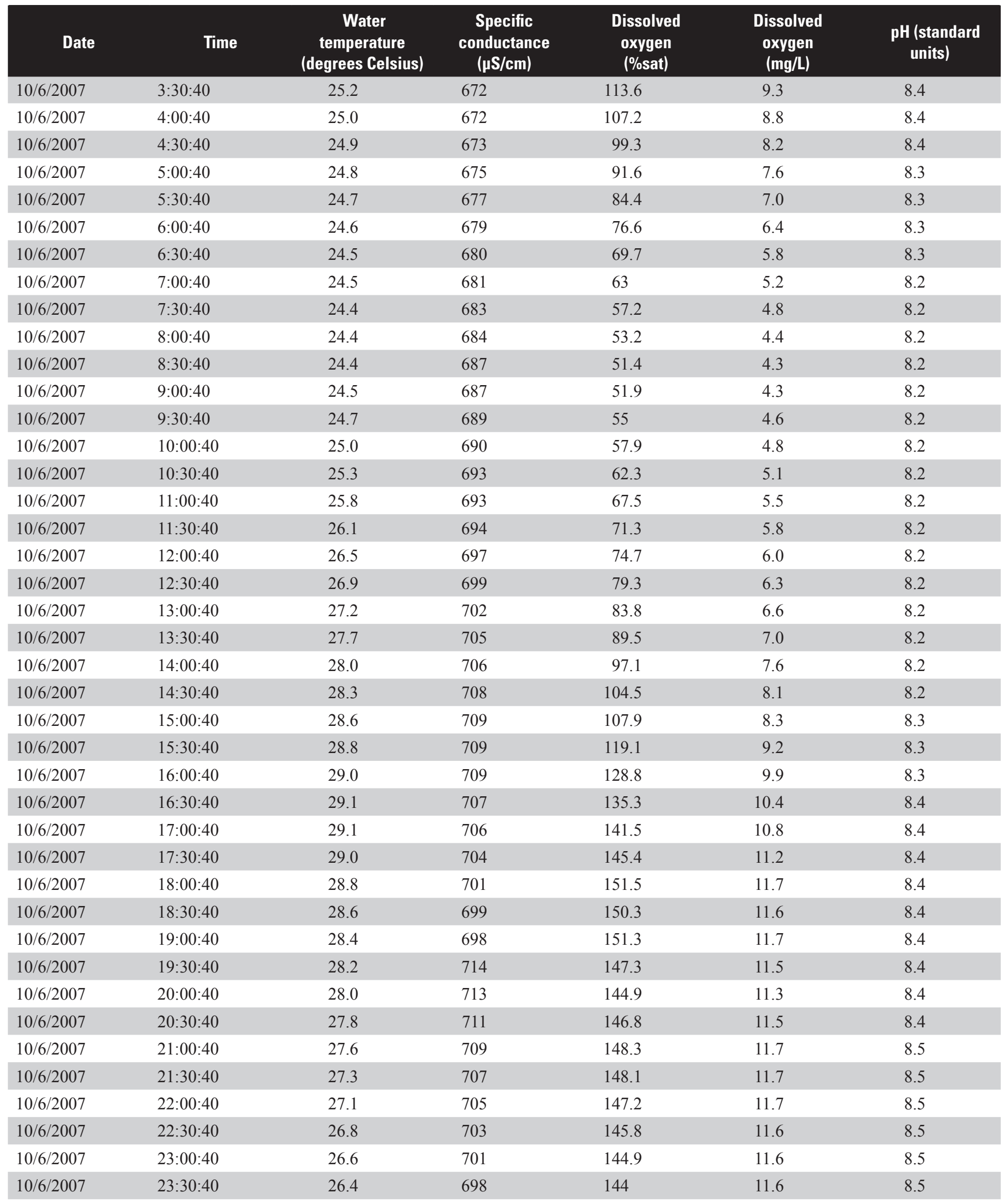


Table 11. Physical property data recorded at 30-minute intervals for station 07288185 Black Bayou at Baltzer, Mississippi, October 4-9, 2007.-Continued

$[\mu \mathrm{S} / \mathrm{cm}$, microsiemens per centimeter; \%sat, percent saturation; mg/L, milligrams per liter; FNU, formazin nephelometric unit; -- , no data]

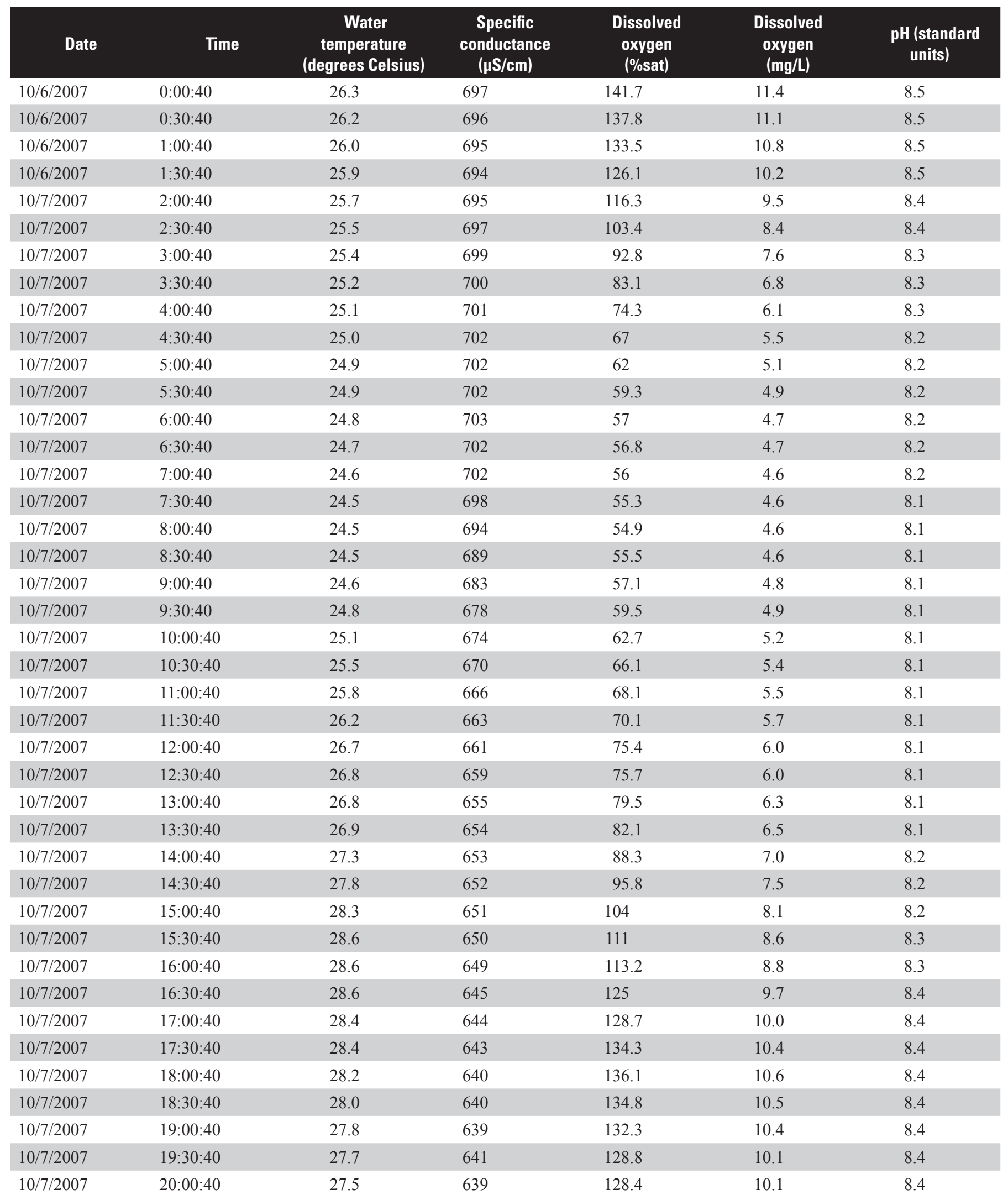


Table 11. Physical property data recorded at 30-minute intervals for station 07288185 Black Bayou at Baltzer, Mississippi, October 4-9, 2007.-Continued

$[\mu \mathrm{S} / \mathrm{cm}$, microsiemens per centimeter; \%sat, percent saturation; mg/L, milligrams per liter; FNU, formazin nephelometric unit; -- , no data]

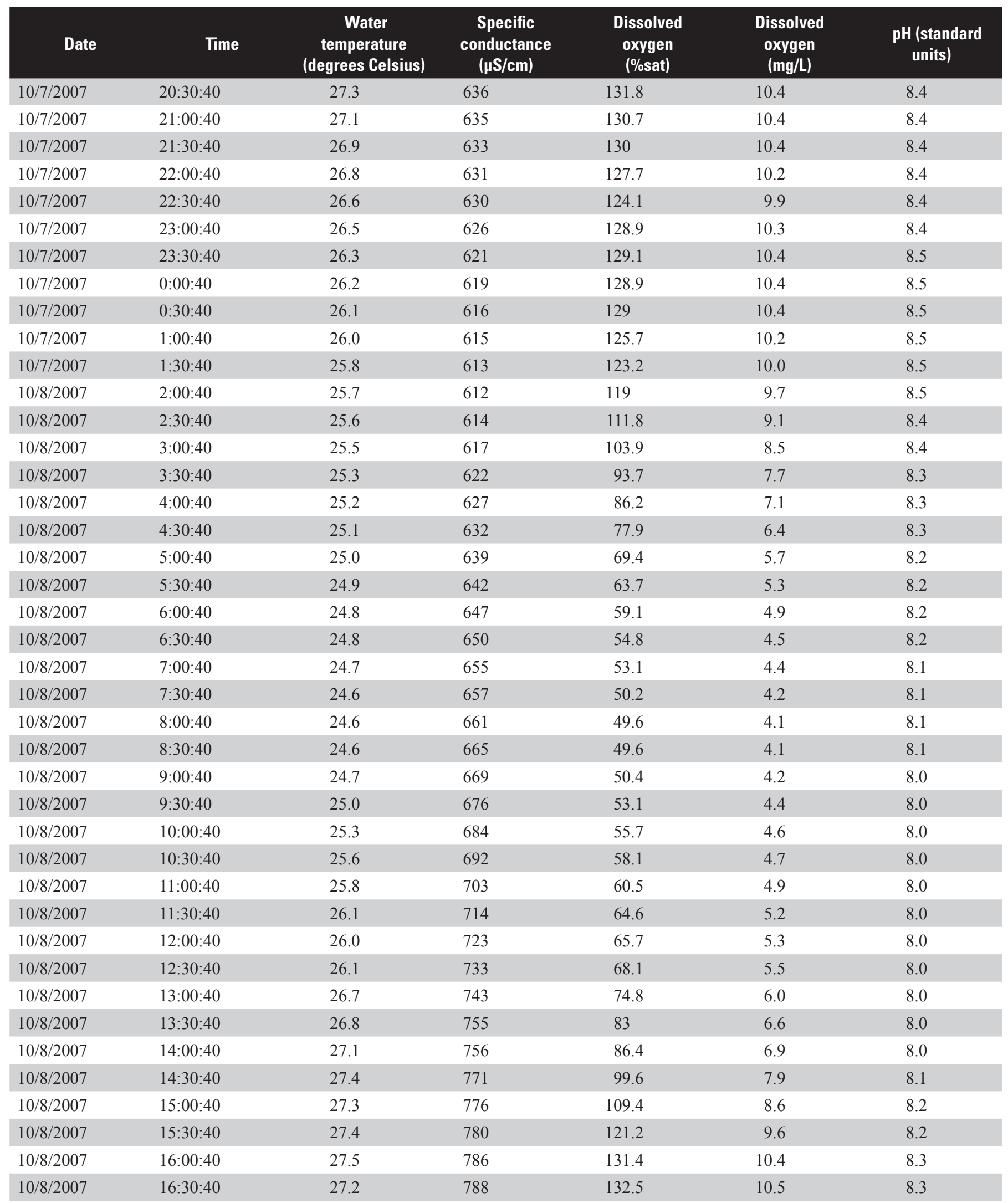


Table 11. Physical property data recorded at 30-minute intervals for station 07288185 Black Bayou at Baltzer, Mississippi, October 4-9, 2007.-Continued

$[\mu \mathrm{S} / \mathrm{cm}$, microsiemens per centimeter; \%sat, percent saturation; mg/L, milligrams per liter; FNU, formazin nephelometric unit; -- , no data]

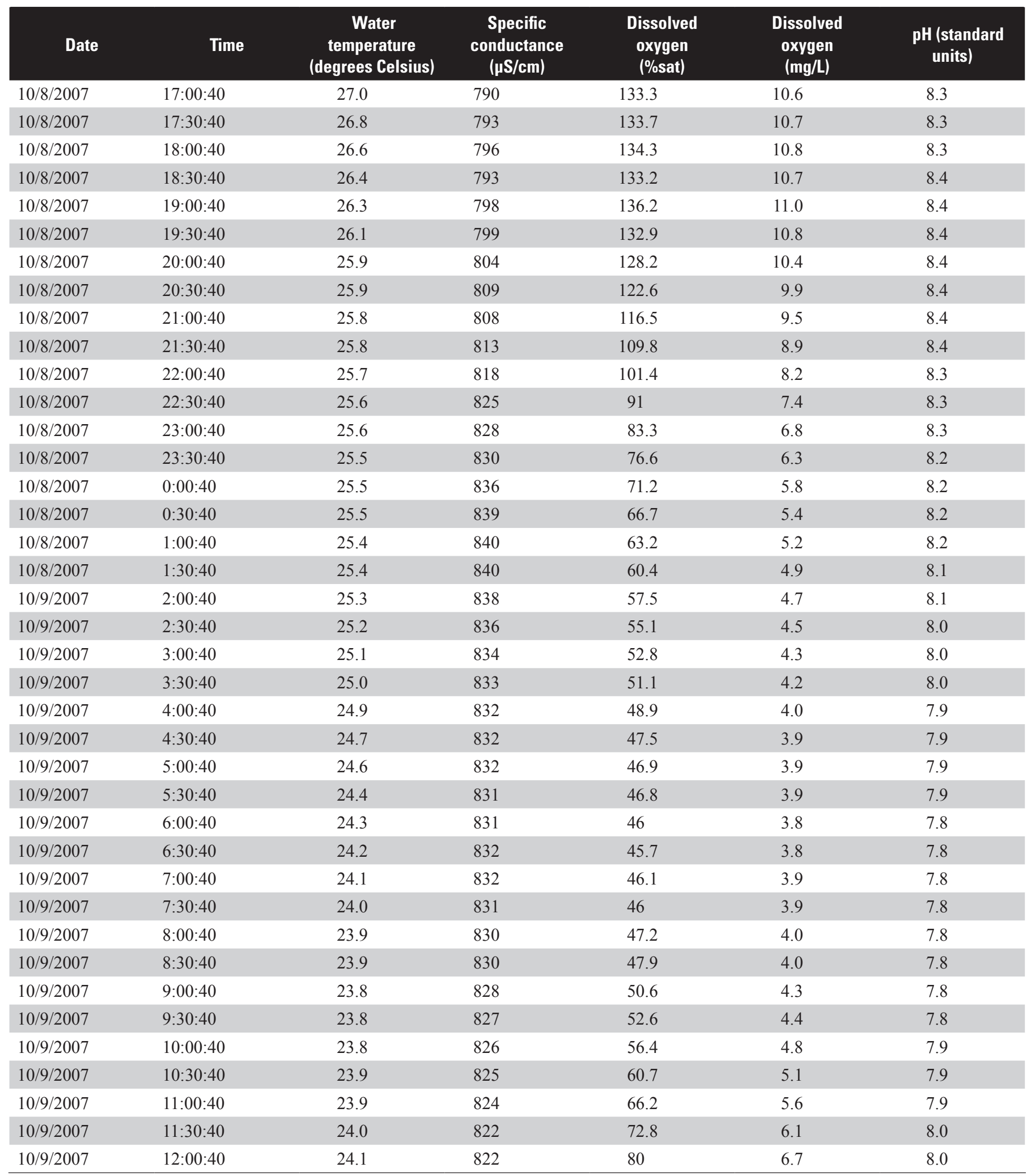


Table 12. Physical property data recorded at 30-minute intervals for station 07288280 Big Sunflower River near Merigold, Mississippi, September 24-27, 2007.

$[\mu \mathrm{S} / \mathrm{cm}$, microsiemens per centimeter; \%sat, percent saturation; mg/L, milligrams per liter; FNU, formazin nephelometric units]

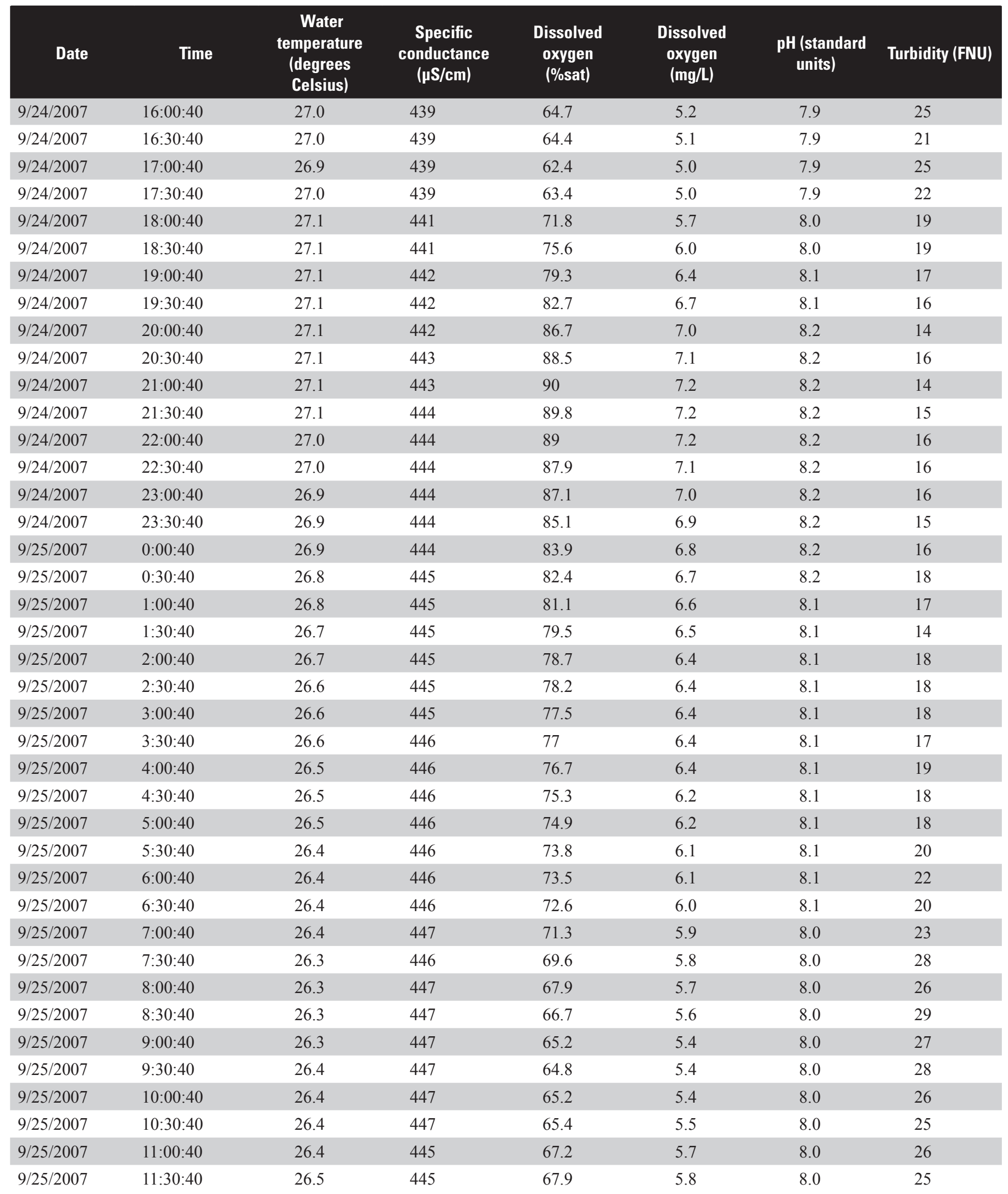


Table 12. Physical property data recorded at 30-minute intervals for station 07288280 Big Sunflower River near Merigold, Mississippi, September 24-27, 2007.-Continued

$[\mu \mathrm{S} / \mathrm{cm}$, microsiemens per centimeter; \%sat, percent saturation; mg/L, milligrams per liter; FNU, formazin nephelometric units]

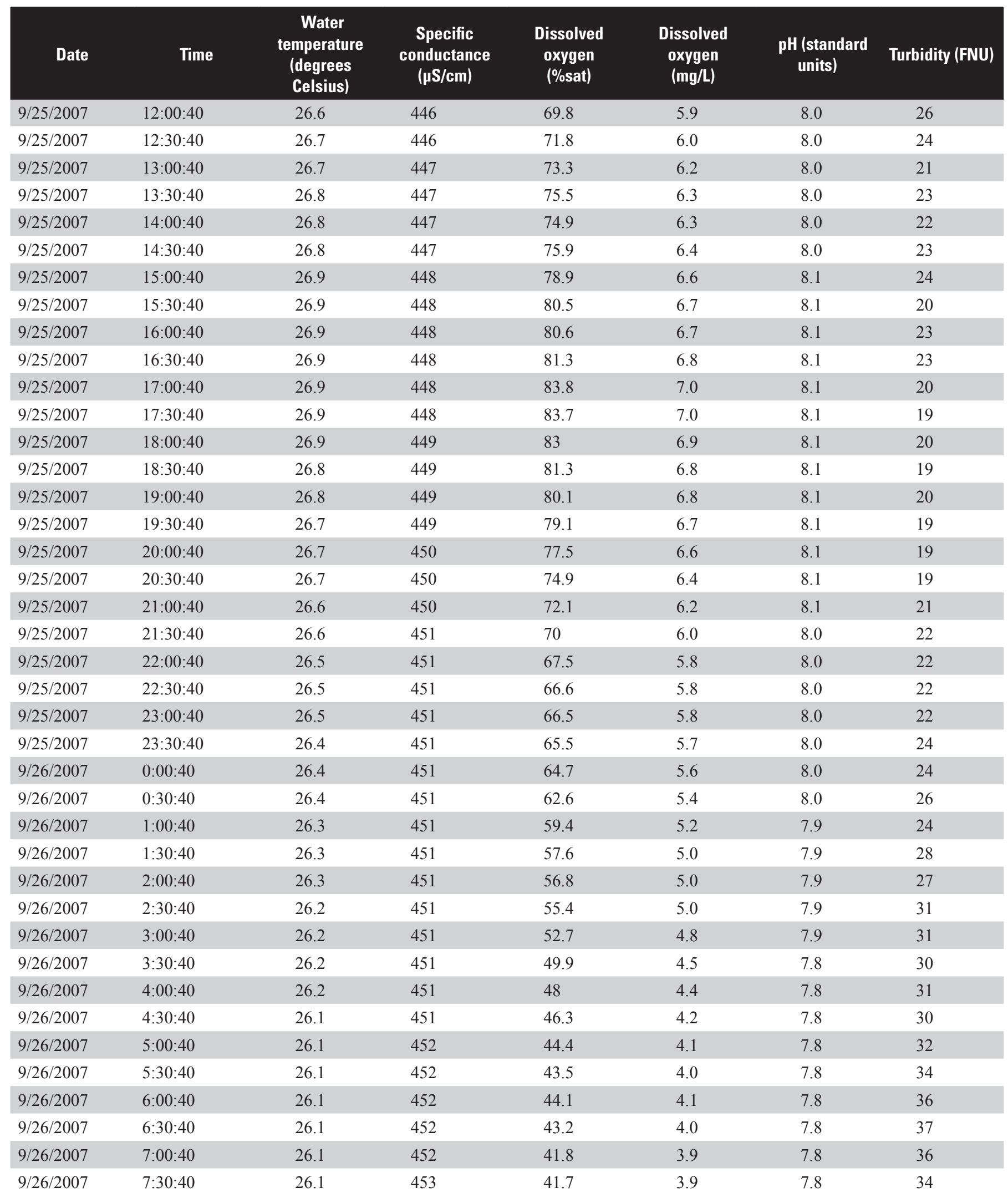


Table 12. Physical property data recorded at 30-minute intervals for station 07288280 Big Sunflower River near Merigold, Mississippi, September 24-27, 2007.-Continued

$[\mu \mathrm{S} / \mathrm{cm}$, microsiemens per centimeter; \%sat, percent saturation; mg/L, milligrams per liter; FNU, formazin nephelometric units $]$

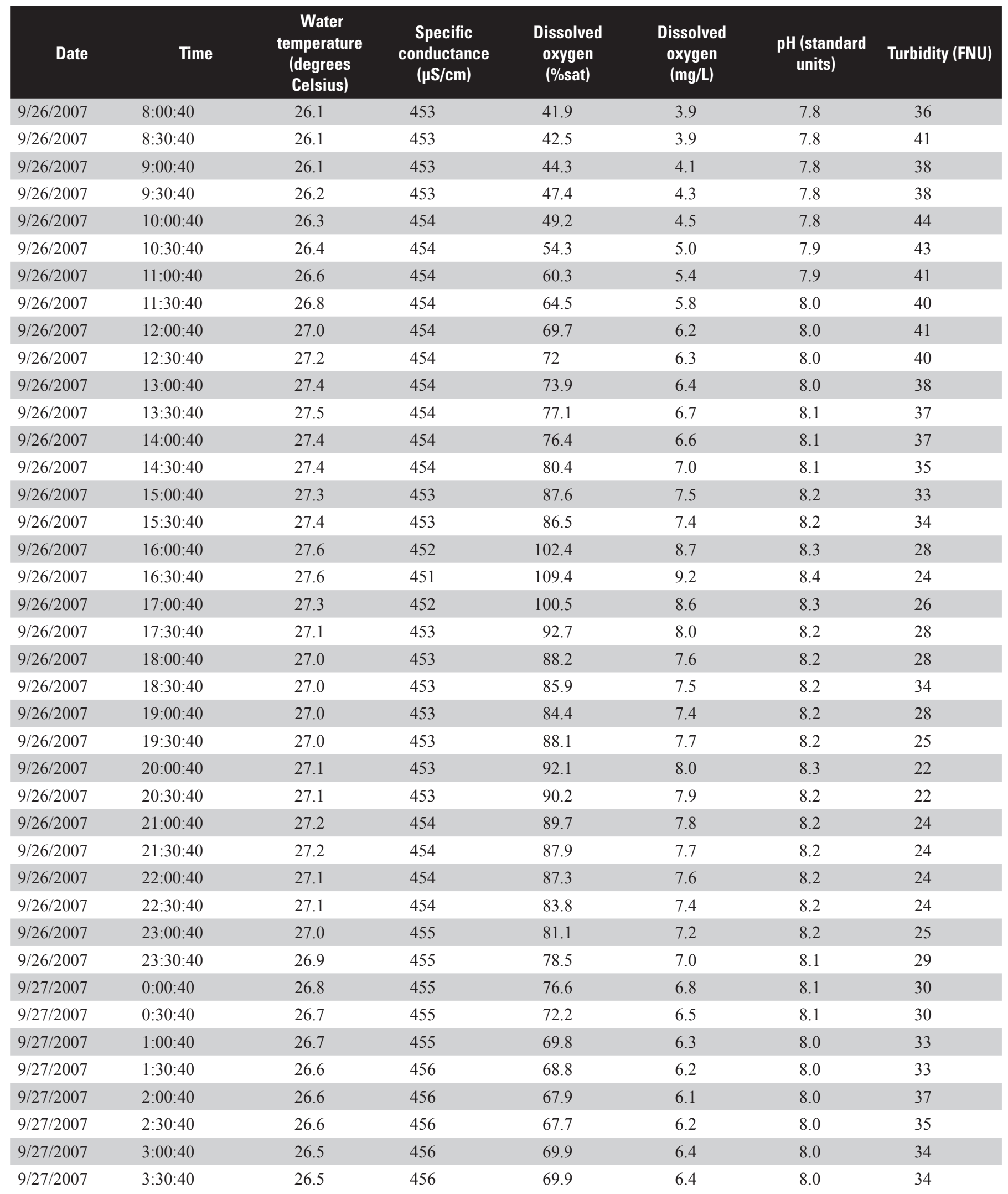


Table 12. Physical property data recorded at 30-minute intervals for station 07288280 Big Sunflower River near Merigold, Mississippi, September 24-27, 2007.-Continued

$[\mu \mathrm{S} / \mathrm{cm}$, microsiemens per centimeter; \%sat, percent saturation; mg/L, milligrams per liter; FNU, formazin nephelometric units]

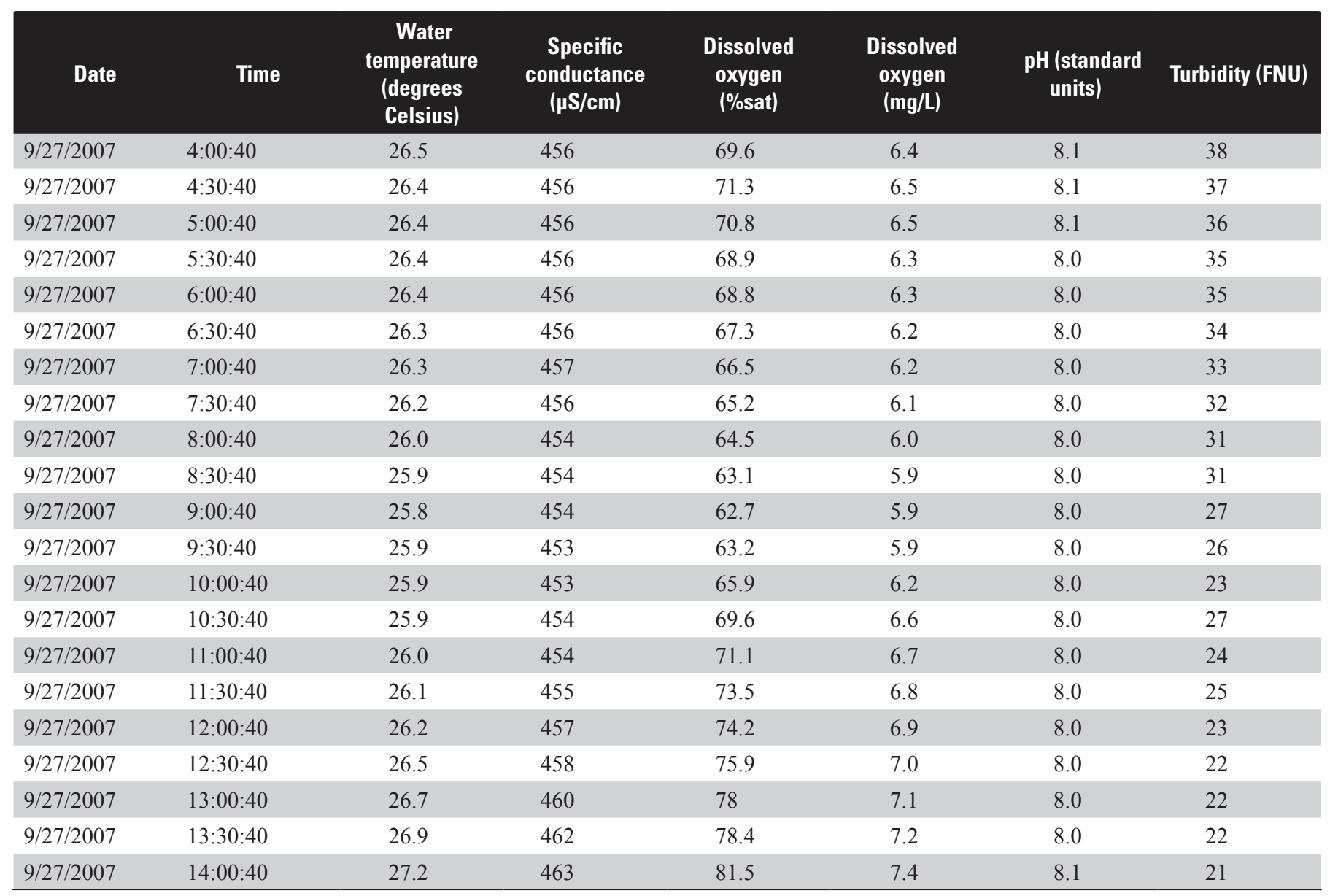


Table 13. Physical property data recorded at 30-minute intervals for station 07288365 Lead Bayou near Boyle, Mississippi, October 1-3, 2007.

$[\mu \mathrm{S} / \mathrm{cm}$, microsiemens per centimeter; \%sat, percent saturation; mg/L, milligrams per liter; FNU, formazin nephelometric units]

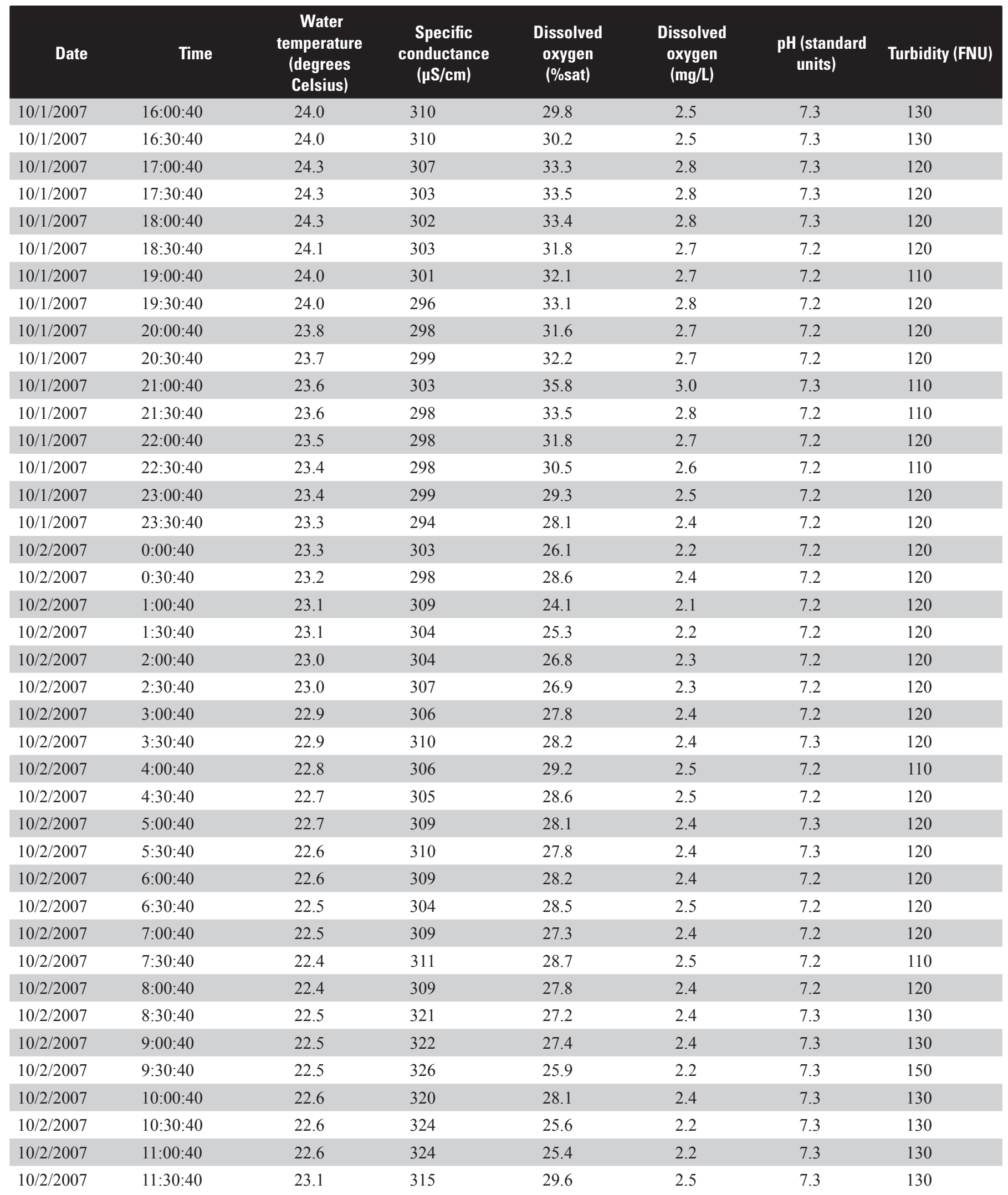


Table 13. Physical property data recorded at 30-minute intervals for station 07288365 Lead Bayou near Boyle, Mississippi, October 1-3, 2007.-Continued

[ $\mu \mathrm{S} / \mathrm{cm}$, microsiemens per centimeter; \%sat, percent saturation; mg/L, milligrams per liter; FNU, formazin nephelometric units]

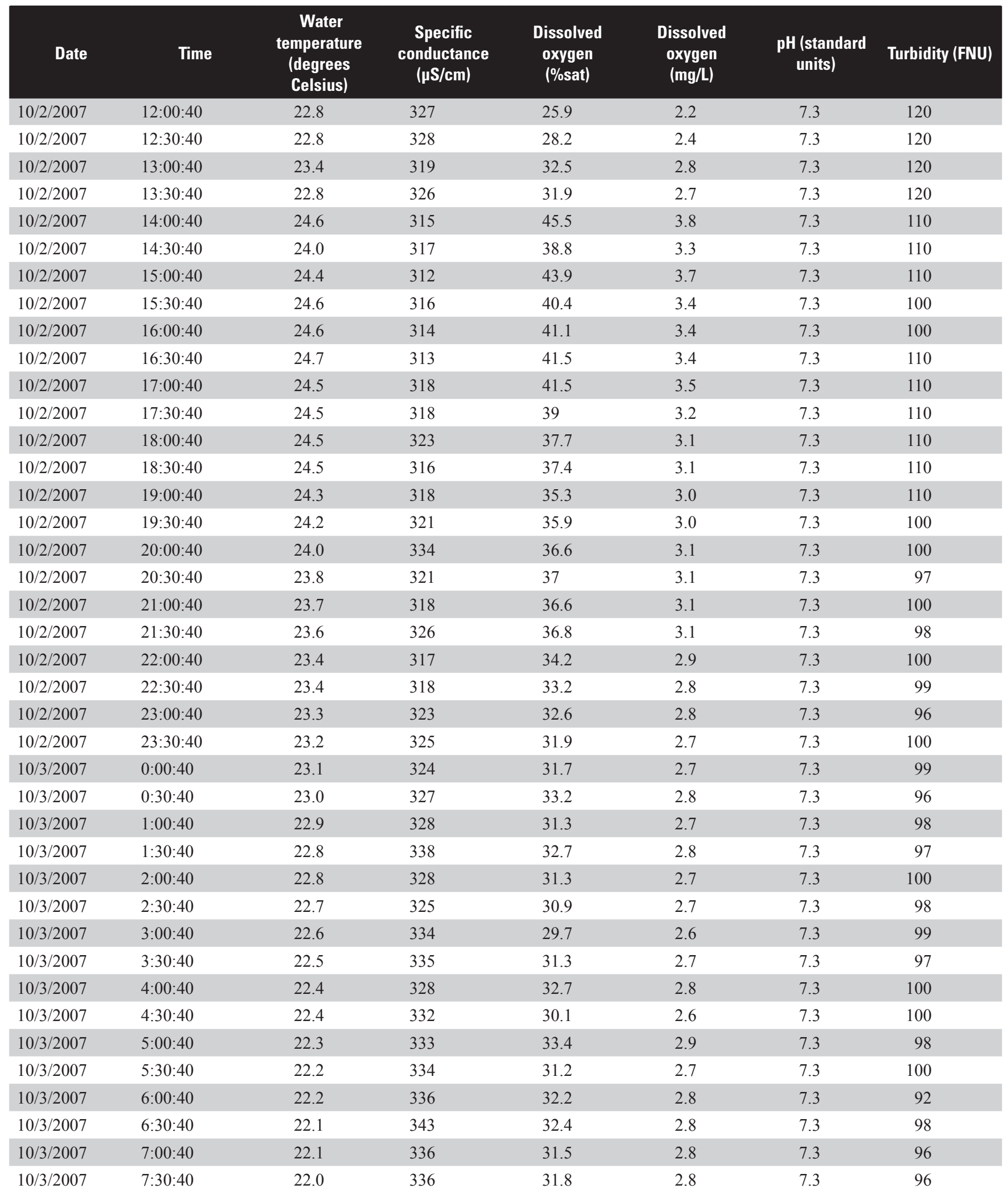


Table 13. Physical property data recorded at 30-minute intervals for station 07288365 Lead Bayou near Boyle, Mississippi, October 1-3, 2007.-Continued

$[\mu \mathrm{S} / \mathrm{cm}$, microsiemens per centimeter; \%sat, percent saturation; mg/L, milligrams per liter; FNU, formazin nephelometric units]

\begin{tabular}{|c|c|c|c|c|c|c|c|}
\hline Date & Time & $\begin{array}{c}\text { Water } \\
\text { temperature } \\
\text { (degrees } \\
\text { Celsius) }\end{array}$ & $\begin{array}{c}\text { Specific } \\
\text { conductance } \\
\text { (pS/cm) }\end{array}$ & $\begin{array}{c}\text { Dissolved } \\
\text { oxygen } \\
\text { (\%sat) }\end{array}$ & $\begin{array}{c}\text { Dissolved } \\
\text { oxygen } \\
\text { (mg/L) }\end{array}$ & $\begin{array}{l}\text { pH (standard } \\
\text { units) }\end{array}$ & Turbidity (FNU) \\
\hline $10 / 3 / 2007$ & $8: 30: 40$ & 22.1 & 337 & 31.6 & 2.8 & 7.3 & 100 \\
\hline $10 / 3 / 2007$ & 9:00:40 & 22.1 & 337 & 32.8 & 2.9 & 7.3 & 100 \\
\hline $10 / 3 / 2007$ & $10: 00: 40$ & 22.3 & 341 & 32.2 & 2.8 & 7.3 & 110 \\
\hline $10 / 3 / 2007$ & $10: 30: 40$ & 22.3 & 348 & 28.1 & 2.4 & 7.3 & 110 \\
\hline $10 / 3 / 2007$ & $11: 00: 40$ & 22.2 & 351 & 28.2 & 2.4 & 7.3 & 120 \\
\hline $10 / 3 / 2007$ & $11: 30: 40$ & 22.6 & 342 & 29.9 & 2.6 & 7.3 & 110 \\
\hline $10 / 3 / 2007$ & $12: 00: 40$ & 22.6 & 350 & 30.4 & 2.6 & 7.3 & 120 \\
\hline $10 / 3 / 2007$ & $14: 00: 40$ & 23.1 & 348 & 34.7 & 3.0 & 7.3 & 110 \\
\hline $10 / 3 / 2007$ & $14: 30: 40$ & 22.9 & 360 & 29.9 & 2.6 & 7.4 & 110 \\
\hline $10 / 3 / 2007$ & $15: 00: 40$ & 23.6 & 354 & 34.4 & 2.9 & 7.4 & 110 \\
\hline $10 / 3 / 2007$ & $15: 30: 40$ & 24.2 & 346 & 40.8 & 3.4 & 7.4 & 110 \\
\hline $10 / 3 / 2007$ & $16: 00: 40$ & 24.4 & 342 & 42.4 & 3.5 & 7.3 & 100 \\
\hline $10 / 3 / 2007$ & $16: 30: 40$ & 24.5 & 339 & 44.5 & 3.7 & 7.4 & 99 \\
\hline $10 / 3 / 2007$ & $17: 00: 40$ & 24.8 & 343 & 48.6 & 4.0 & 7.4 & 88 \\
\hline $10 / 3 / 2007$ & $17: 30: 40$ & 24.3 & 348 & 44 & 3.7 & 7.4 & 91 \\
\hline
\end{tabular}


Table 14. Physical property data recorded at 30-minute intervals for station 07288600 Quiver River near Moorhead, Mississippi, October 1-3, 2007.

$[\mu \mathrm{S} / \mathrm{cm}$, microsiemens per centimeter; \%sat, percent saturation; mg/L, milligrams per liter; FNU, formazin nephelometric unit; -- , no data]

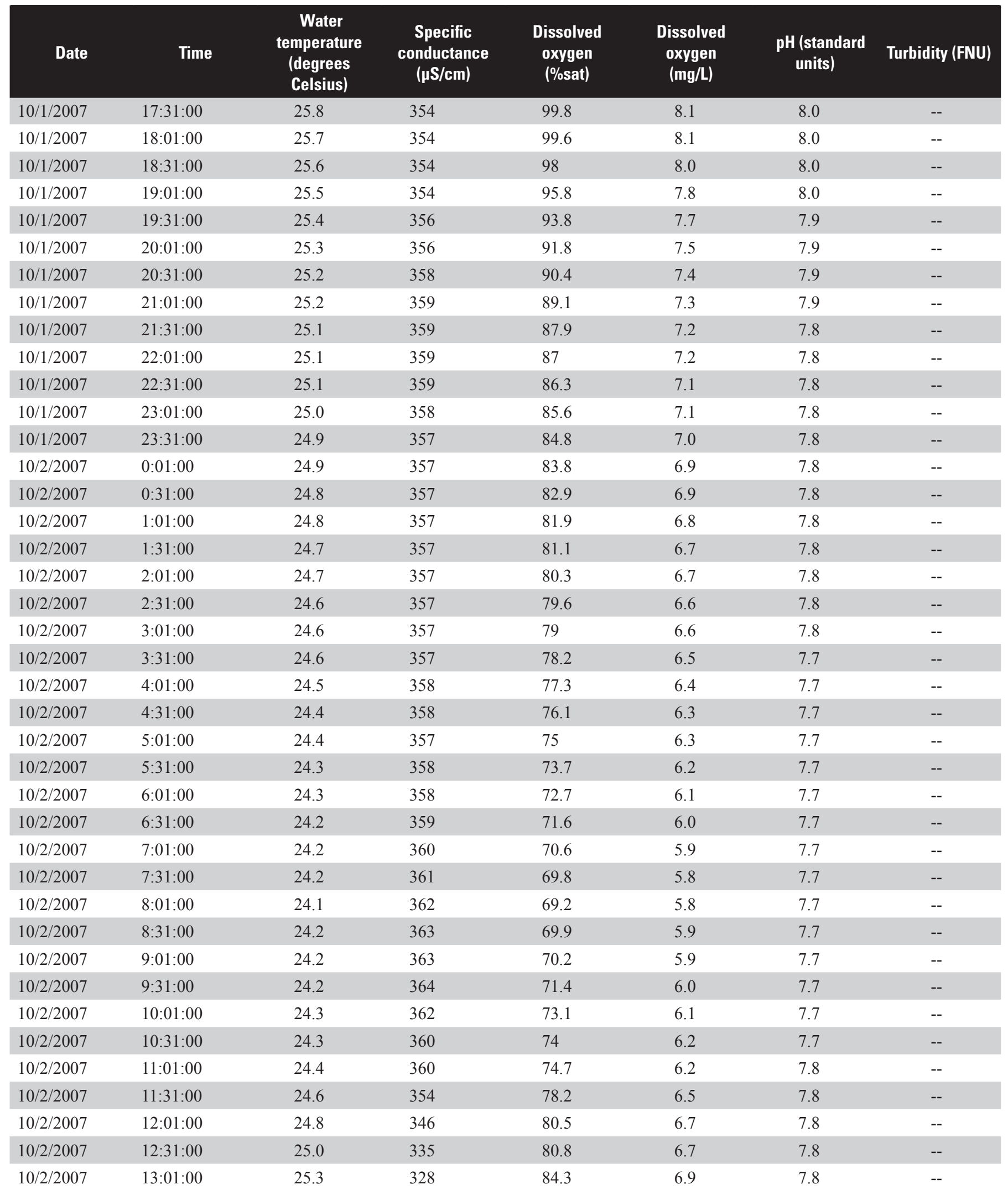


Table 14. Physical property data recorded at 30-minute intervals for station 07288600 Quiver River near Moorhead, Mississippi, October 1-3, 2007.-Continued

$[\mu \mathrm{S} / \mathrm{cm}$, microsiemens per centimeter; \%sat, percent saturation; mg/L, milligrams per liter; FNU, formazin nephelometric unit; -- , no data]

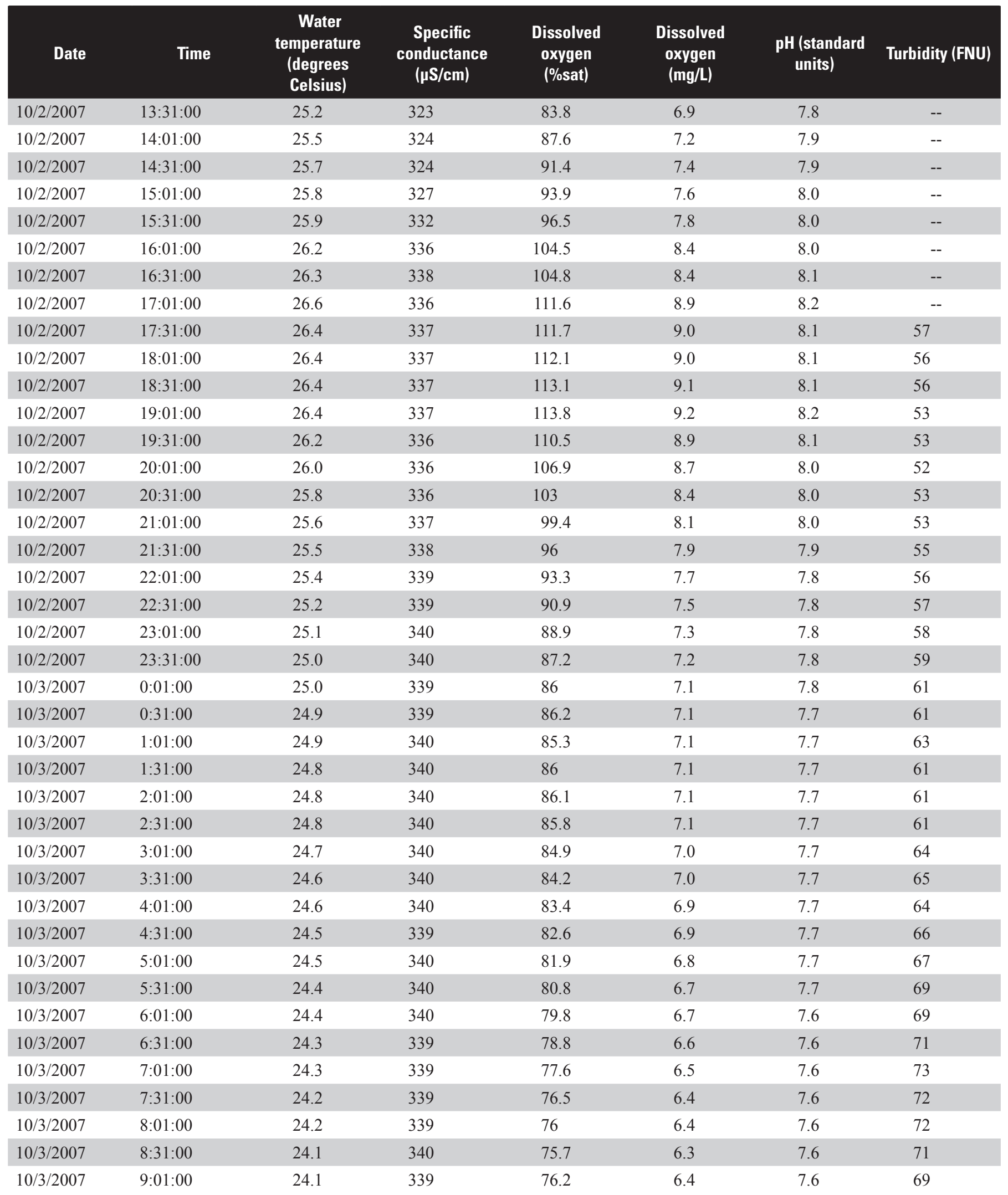


Table 14. Physical property data recorded at 30-minute intervals for station 07288600 Quiver River near Moorhead, Mississippi, October 1-3, 2007.-Continued

$[\mu \mathrm{S} / \mathrm{cm}$, microsiemens per centimeter; \%sat, percent saturation; mg/L, milligrams per liter; FNU, formazin nephelometric unit; -- , no data]

\begin{tabular}{|c|c|c|c|c|c|c|c|}
\hline Date & Time & $\begin{array}{c}\text { Water } \\
\text { temperature } \\
\text { (degrees } \\
\text { Celsius) }\end{array}$ & $\begin{array}{c}\text { Specific } \\
\text { conductance } \\
\text { (pS/cm) }\end{array}$ & $\begin{array}{c}\text { Dissolved } \\
\text { oxygen } \\
\text { (\%sat) }\end{array}$ & $\begin{array}{c}\text { Dissolved } \\
\text { oxygen } \\
\text { (mg/L) }\end{array}$ & $\begin{array}{l}\text { pH (standard } \\
\text { units) }\end{array}$ & Turbidity (FNU) \\
\hline $10 / 3 / 2007$ & 10:01:00 & 24.3 & 340 & 78.8 & 6.6 & 7.6 & 68 \\
\hline $10 / 3 / 2007$ & $10: 31: 00$ & 24.4 & 339 & 80.1 & 6.7 & 7.6 & 68 \\
\hline $10 / 3 / 2007$ & $11: 31: 00$ & 24.9 & 337 & 85.8 & 7.1 & 7.7 & 67 \\
\hline $10 / 3 / 2007$ & 12:01:00 & 24.9 & 336 & 86.5 & 7.2 & 7.7 & 68 \\
\hline $10 / 3 / 2007$ & $12: 31: 00$ & 25.2 & 335 & 87.8 & 7.2 & 7.8 & 68 \\
\hline $10 / 3 / 2007$ & 13:01:00 & 25.3 & 334 & 91.1 & 7.5 & 7.8 & 64 \\
\hline $10 / 3 / 2007$ & 15:01:00 & 27.2 & 333 & 121.9 & 9.7 & 8.3 & 56 \\
\hline $10 / 3 / 2007$ & $15: 31: 00$ & 27.5 & 333 & 126.7 & 10.0 & 8.4 & 53 \\
\hline $10 / 3 / 2007$ & 16:01:00 & 27.5 & 333 & 128.1 & 10.1 & 8.4 & 53 \\
\hline $10 / 3 / 2007$ & $16: 31: 00$ & 27.8 & 333 & 134.5 & 10.6 & 8.5 & 50 \\
\hline
\end{tabular}


Table 15. Physical property data recorded at 30-minute intervals for station 07288656 Bogue Phalia near Darlove, Mississippi, October 2-4, 2007.

$[\mu \mathrm{S} / \mathrm{cm}$, microsiemens per centimeter; \%sat, percent saturation; mg/L, milligrams per liter; FNU, formazin nephelometric units]

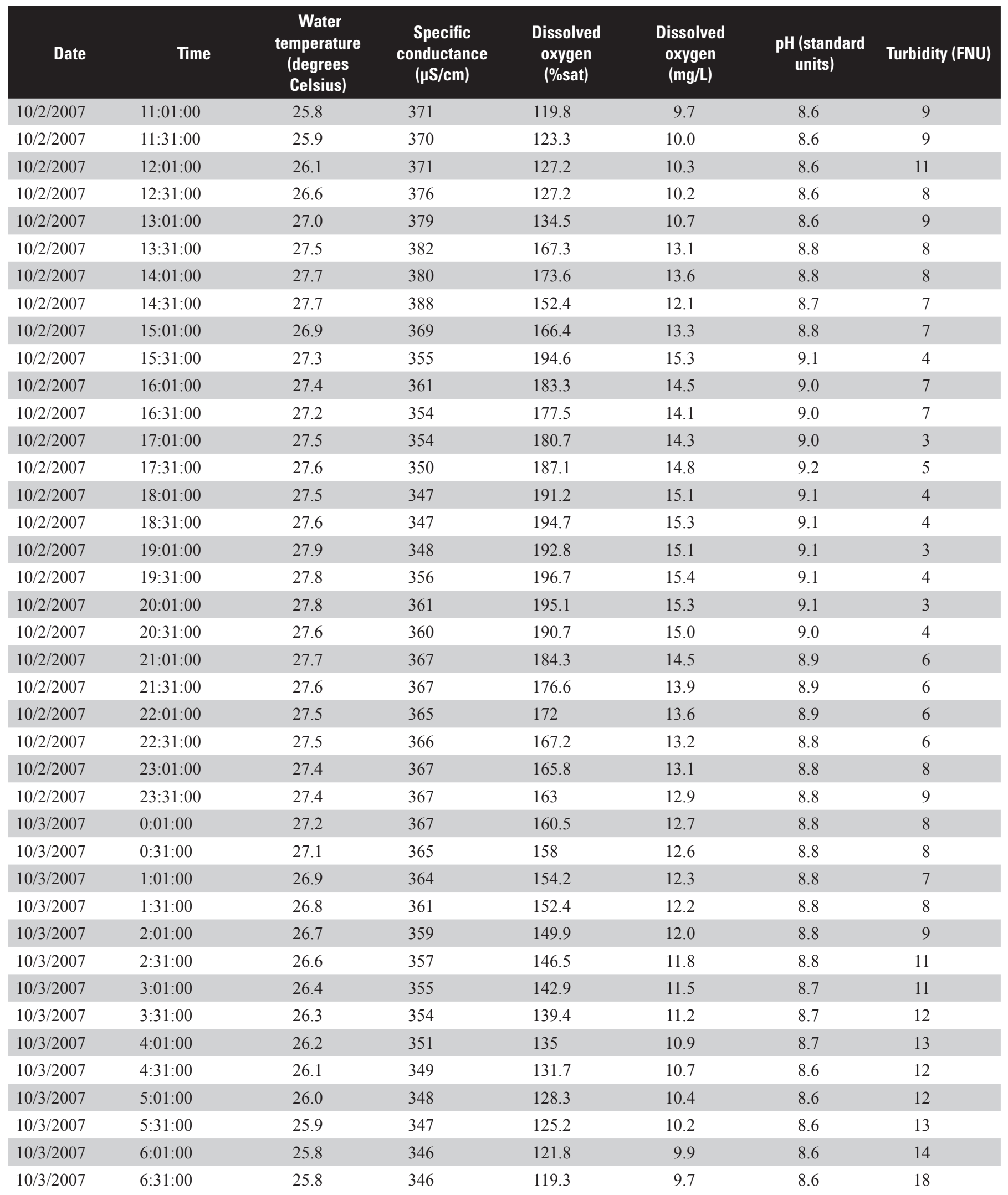


Table 15. Physical property data recorded at 30-minute intervals for station 07288656 Bogue Phalia near Darlove, Mississippi, October 2-4, 2007.-Continued

$[\mu \mathrm{S} / \mathrm{cm}$, microsiemens per centimeter; \%sat, percent saturation; $\mathrm{mg} / \mathrm{L}$, milligrams per liter; FNU, formazin nephelometric units]

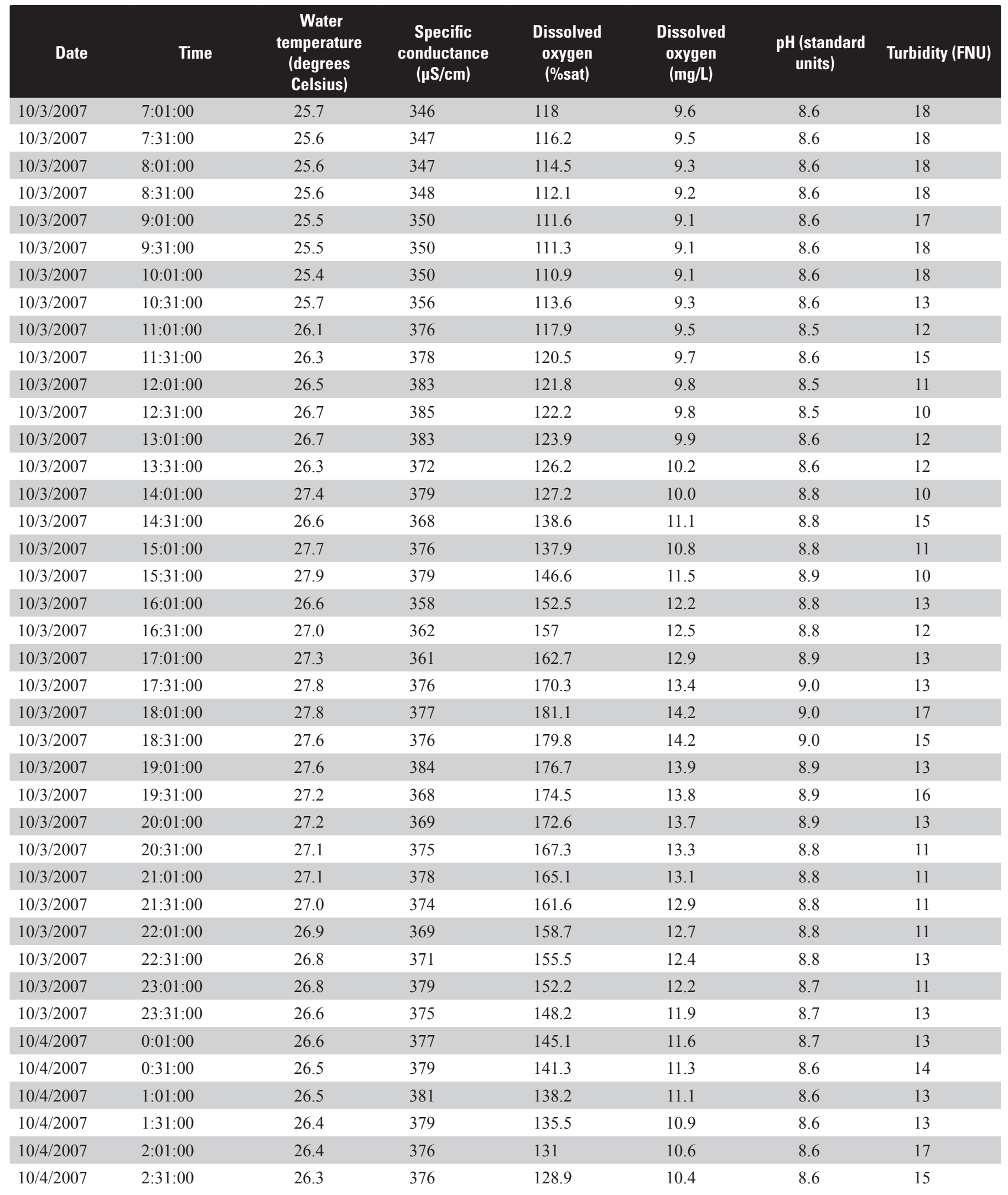


Table 15. Physical property data recorded at 30-minute intervals for station 07288656 Bogue Phalia near Darlove, Mississippi, October 2-4, 2007.-Continued

$[\mu \mathrm{S} / \mathrm{cm}$, microsiemens per centimeter; \%sat, percent saturation; mg/L, milligrams per liter; FNU, formazin nephelometric units]

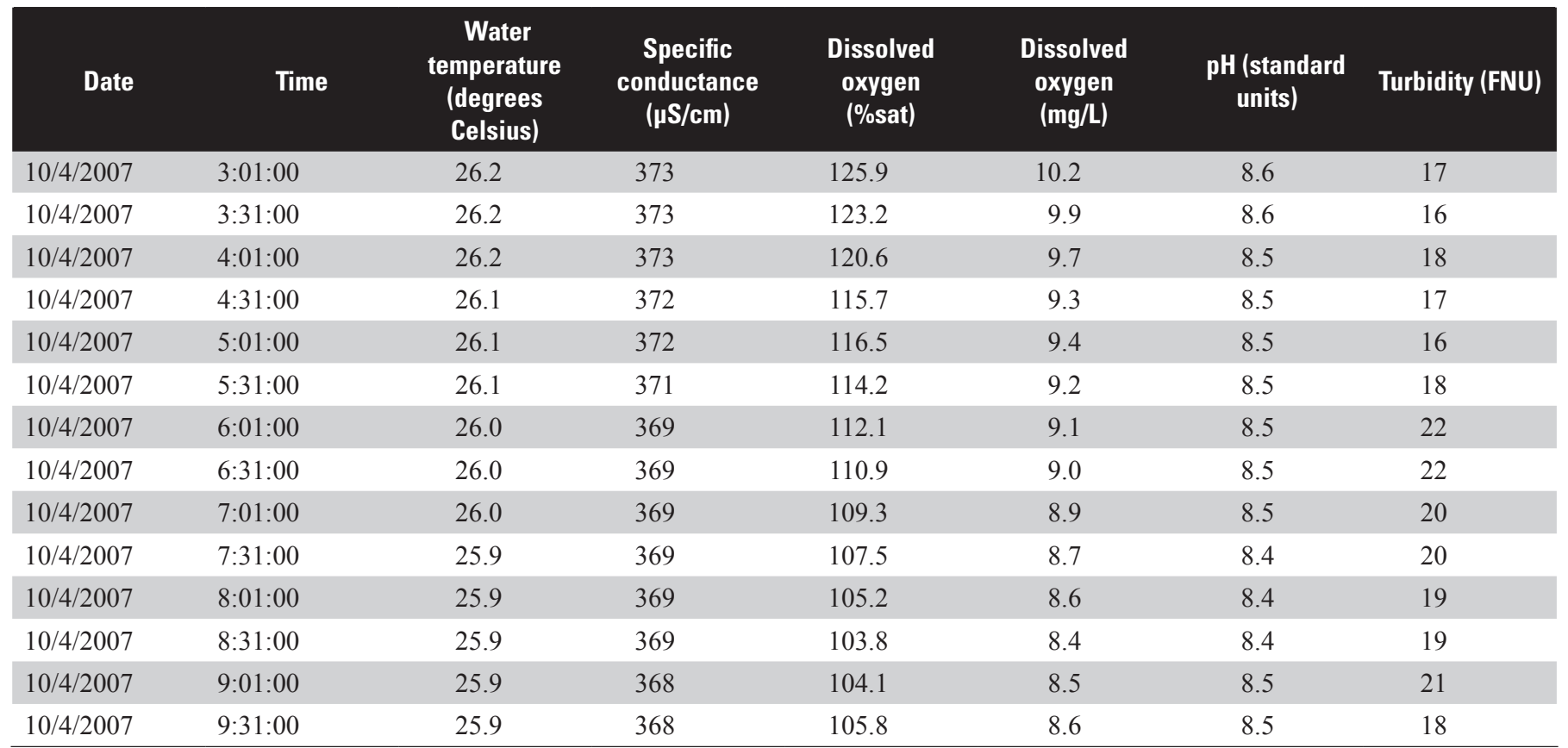


Table 16. Physical property data recorded at 30-minute intervals for station 07288792 Deer Creek northeast of Valley Park, Mississippi, September -Continued

$[\mu \mathrm{S} / \mathrm{cm}$, microsiemens per centimeter; \%sat, percent saturation; mg/L, milligrams per liter; FNU, formazin nephelometric units; -- , no data]

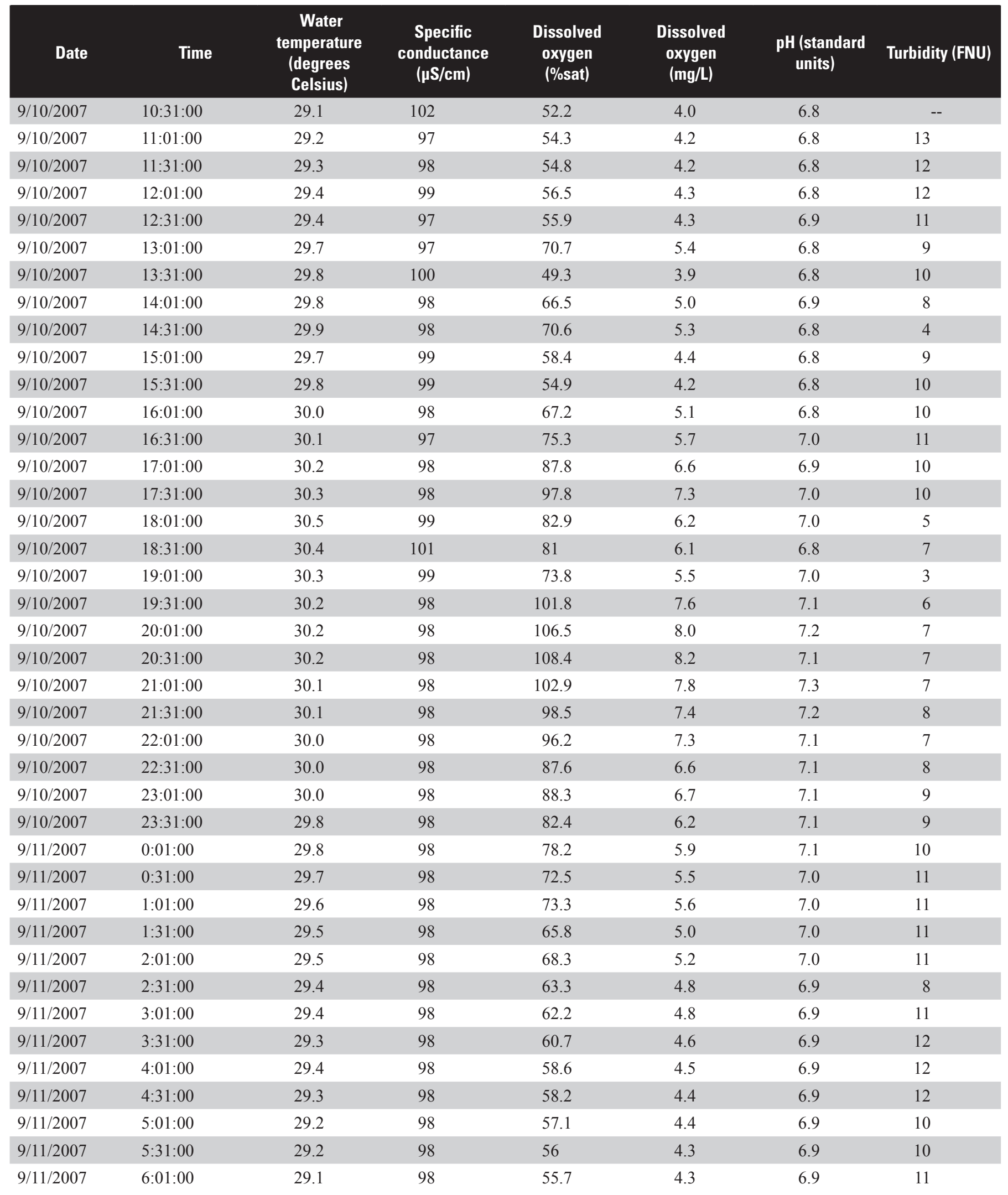


Table 16. Physical property data recorded at 30-minute intervals for station 07288792 Deer Creek northeast of Valley Park, Mississippi, September 10-14, 2007.-Continued

$[\mu \mathrm{S} / \mathrm{cm}$, microsiemens per centimeter; \%sat, percent saturation; mg/L, milligrams per liter; FNU, formazin nephelometric units; -- , no data]

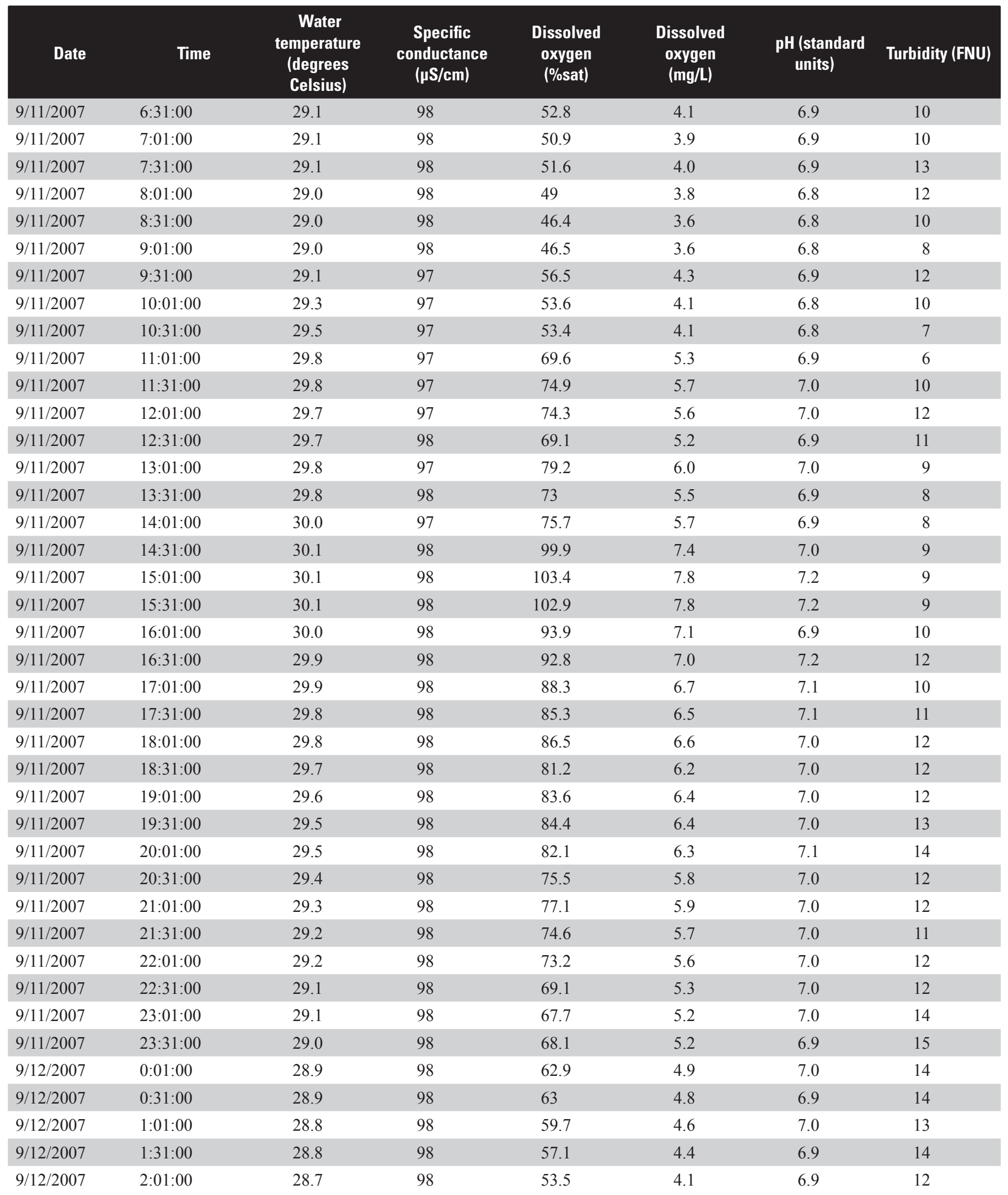


Table 16. Physical property data recorded at 30-minute intervals for station 07288792 Deer Creek northeast of Valley Park, Mississippi, September 10-14, 2007.-Continued

$[\mu \mathrm{S} / \mathrm{cm}$, microsiemens per centimeter; \%sat, percent saturation; mg/L, milligrams per liter; FNU, formazin nephelometric units; -- , no data]

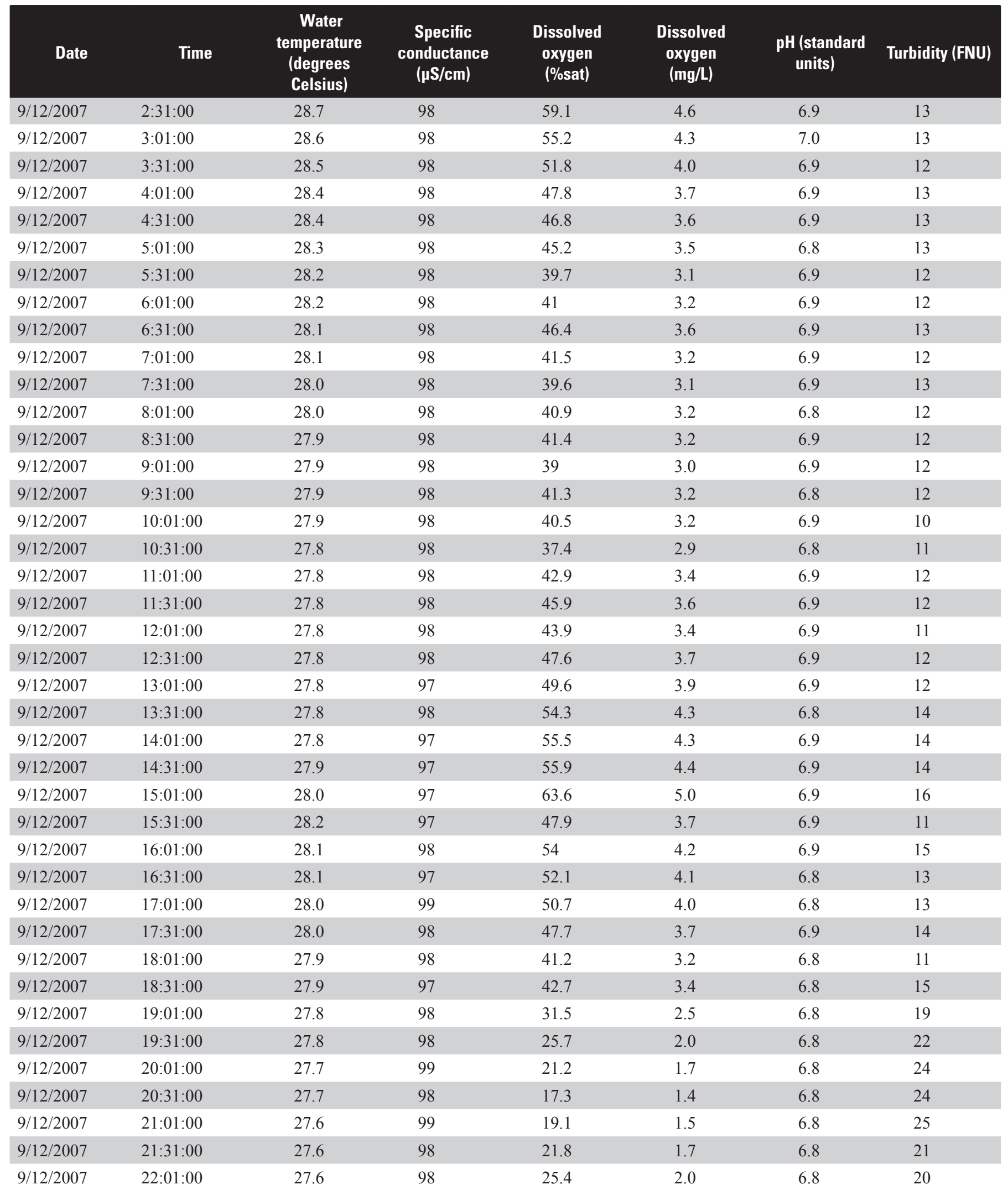


Table 16. Physical property data recorded at 30-minute intervals for station 07288792 Deer Creek northeast of Valley Park, Mississippi, September 10-14, 2007.-Continued

$[\mu \mathrm{S} / \mathrm{cm}$, microsiemens per centimeter; \%sat, percent saturation; mg/L, milligrams per liter; FNU, formazin nephelometric units; -- , no data]

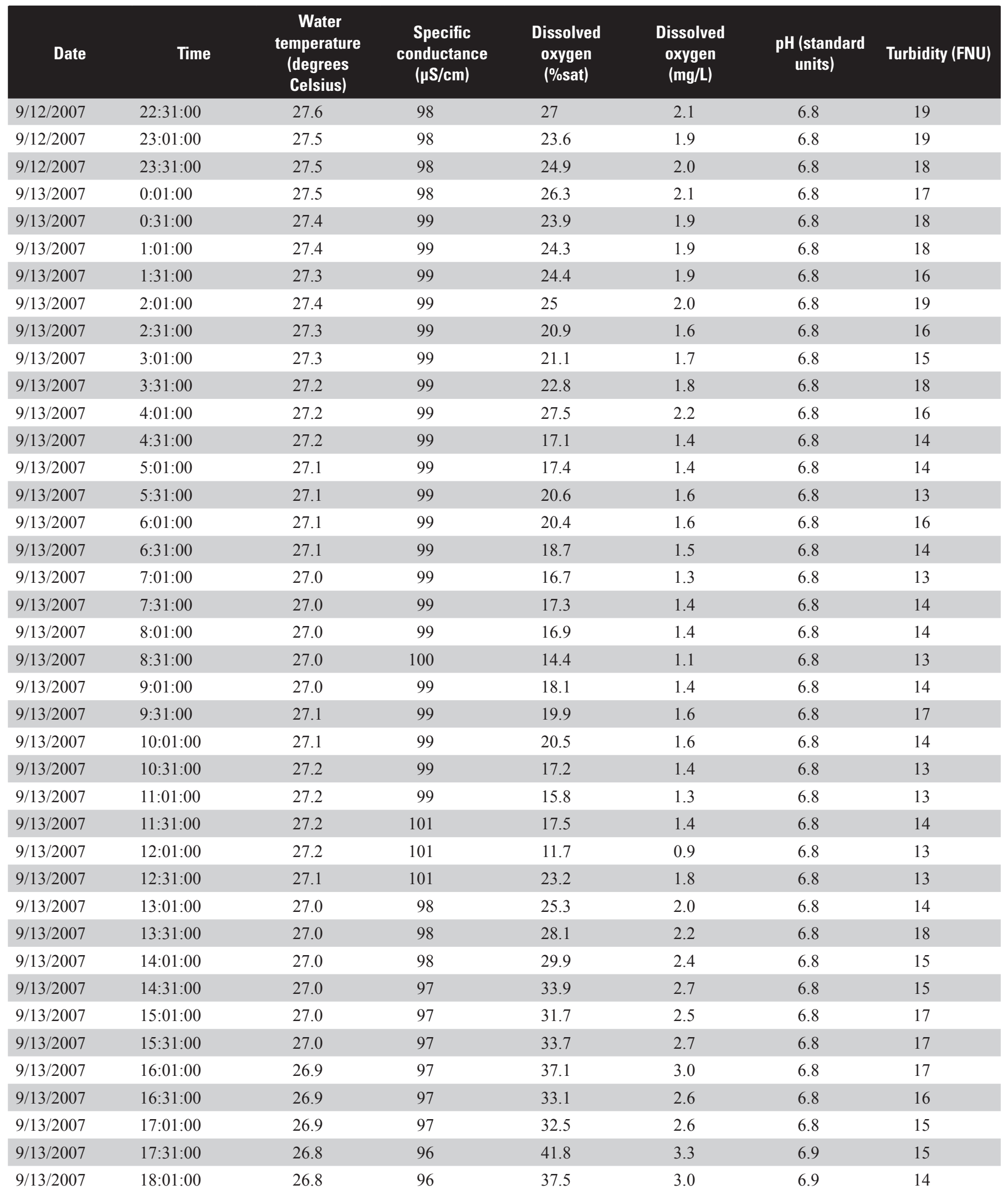


Table 16. Physical property data recorded at 30-minute intervals for station 07288792 Deer Creek northeast of Valley Park, Mississippi, September 10-14, 2007.-Continued

$[\mu \mathrm{S} / \mathrm{cm}$, microsiemens per centimeter; \%sat, percent saturation; $\mathrm{mg} / \mathrm{L}$, milligrams per liter; FNU, formazin nephelometric units; -- , no data]

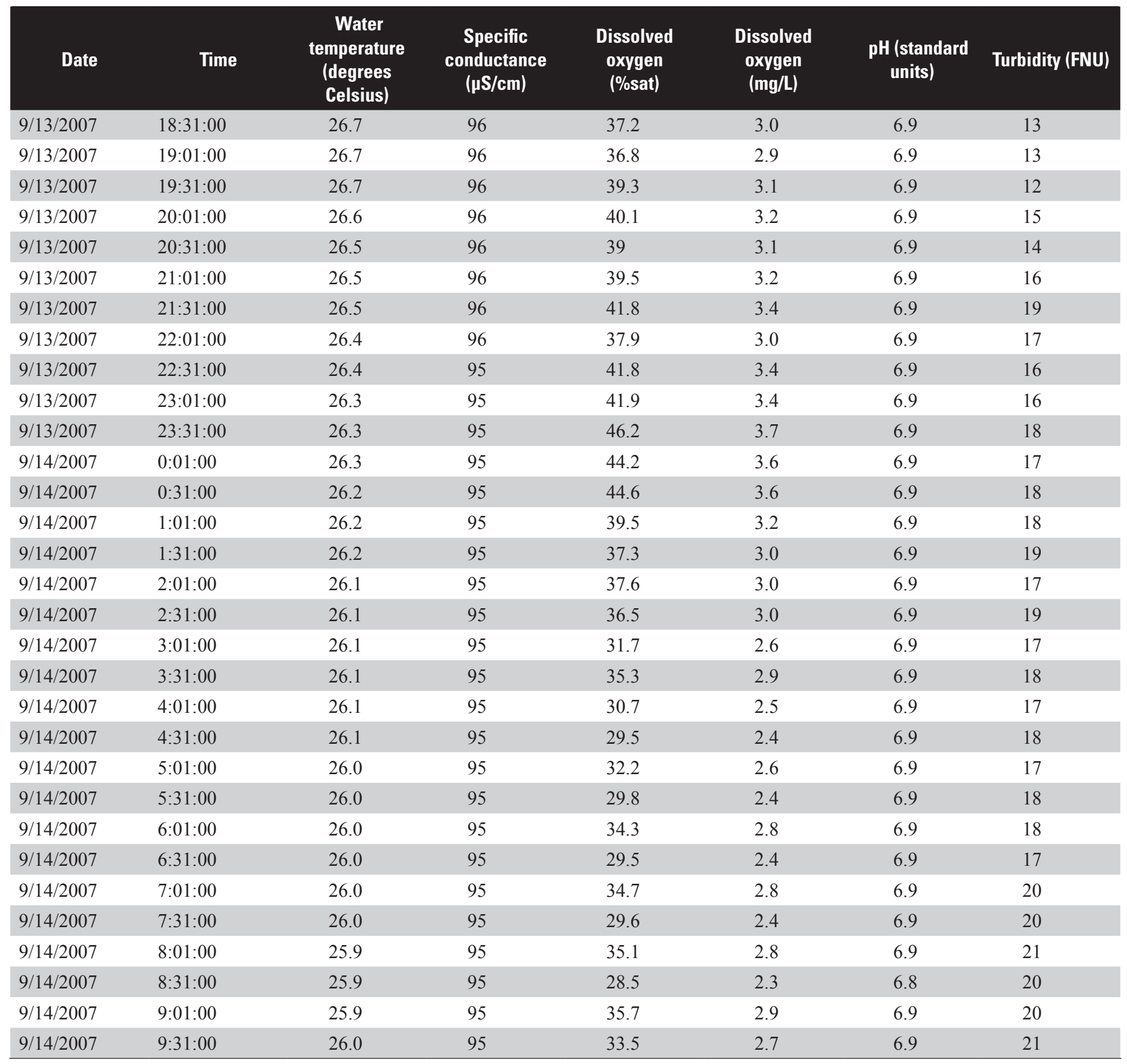


Table 17. Physical property data recorded at 30-minute intervals for station 325901090465500 Jaynes Bayou at Mouth near Anguilla, Mississippi, September 10-13, 2007.

$[\mu \mathrm{S} / \mathrm{cm}$, microsiemens per centimeter; \%sat, percent saturation; mg/L, milligrams per liter; FNU, formazin nephelometric units; -- , no data]

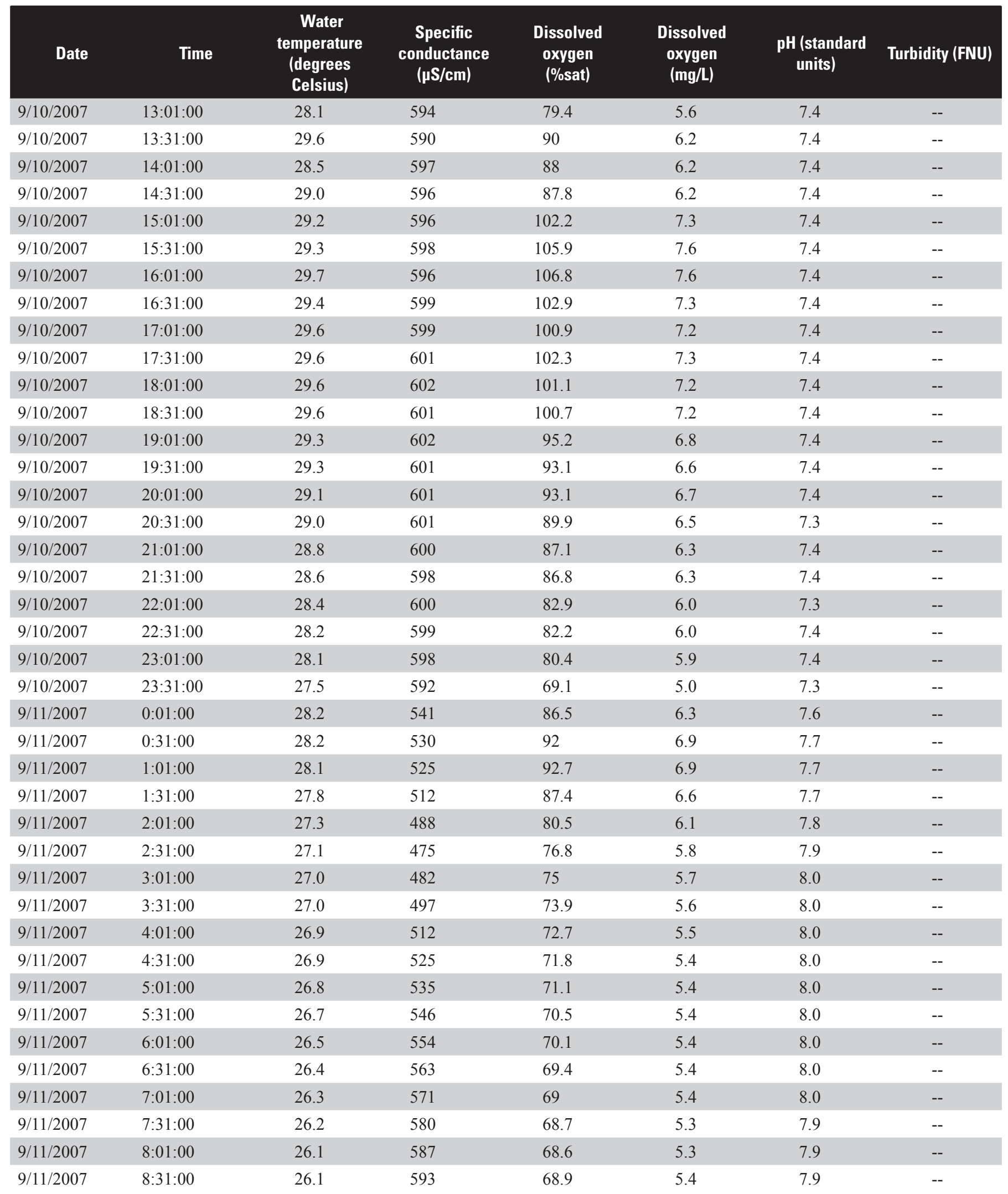


Table 17. Physical property data recorded at 30-minute intervals for station 325901090465500 Jaynes Bayou at Mouth near Anguilla, Mississippi, September 10-13, 2007.-Continued

$[\mu \mathrm{S} / \mathrm{cm}$, microsiemens per centimeter; \%sat, percent saturation; $\mathrm{mg} / \mathrm{L}$, milligrams per liter; FNU, formazin nephelometric units; -- , no data]

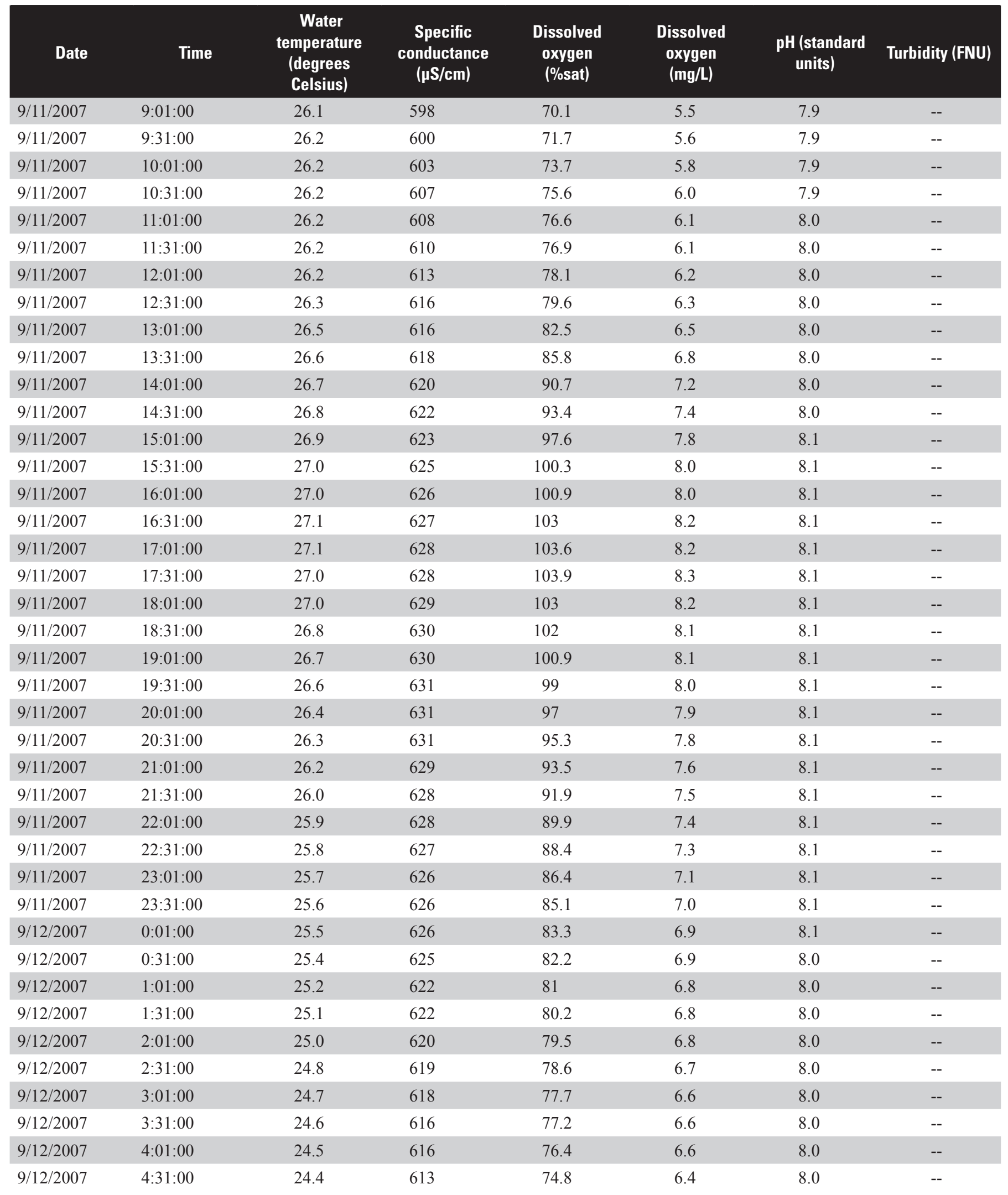


Table 17. Physical property data recorded at 30-minute intervals for station 325901090465500 Jaynes Bayou at Mouth near Anguilla, Mississippi, September 10-13, 2007.-Continued

$[\mu \mathrm{S} / \mathrm{cm}$, microsiemens per centimeter; \%sat, percent saturation; mg/L, milligrams per liter; FNU, formazin nephelometric units; -- , no data]

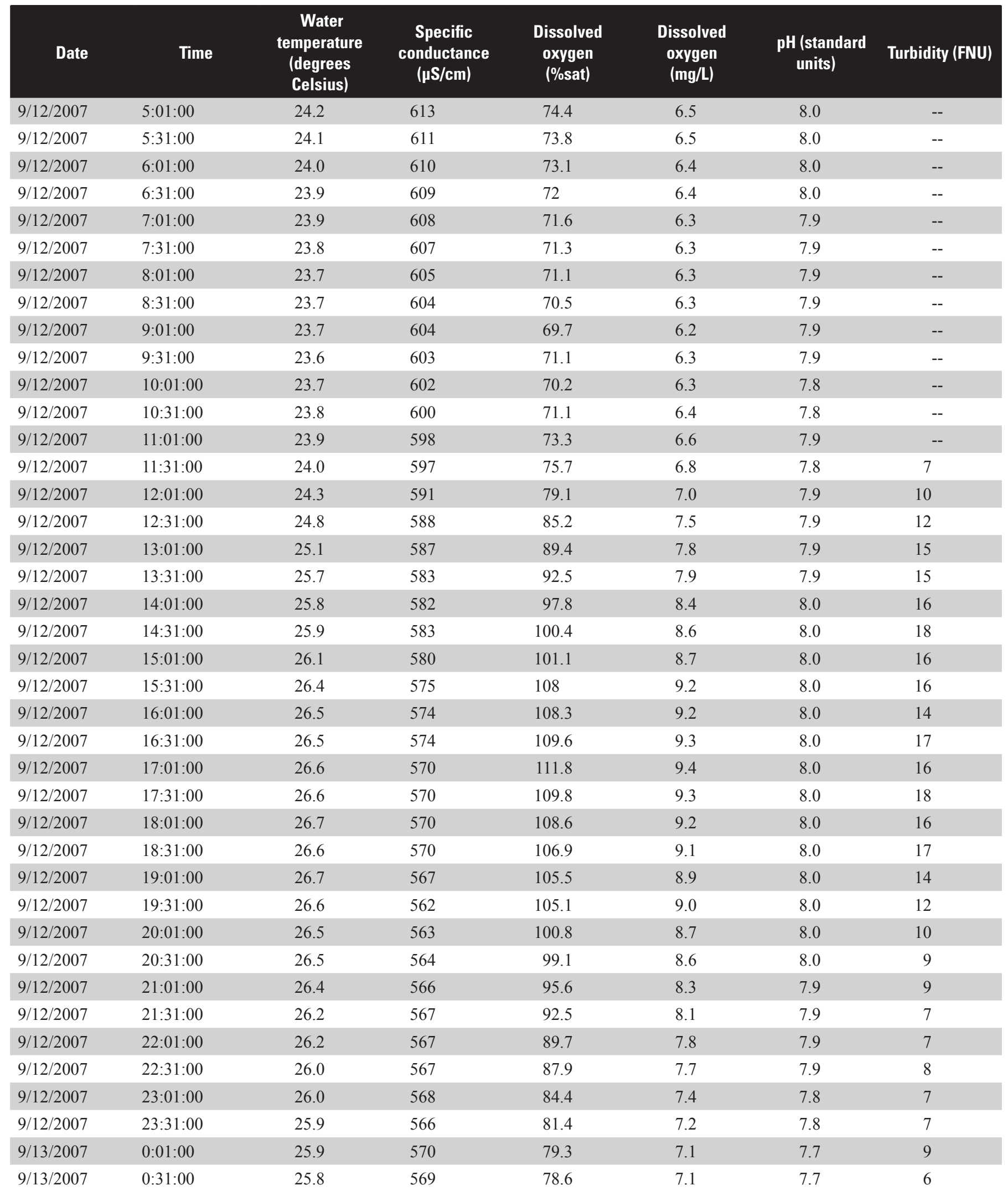


Table 17. Physical property data recorded at 30-minute intervals for station 325901090465500 Jaynes Bayou at Mouth near Anguilla, Mississippi, September 10-13, 2007.-Continued

$[\mu \mathrm{S} / \mathrm{cm}$, microsiemens per centimeter; \%sat, percent saturation; $\mathrm{mg} / \mathrm{L}$, milligrams per liter; FNU, formazin nephelometric units; -- , no data]

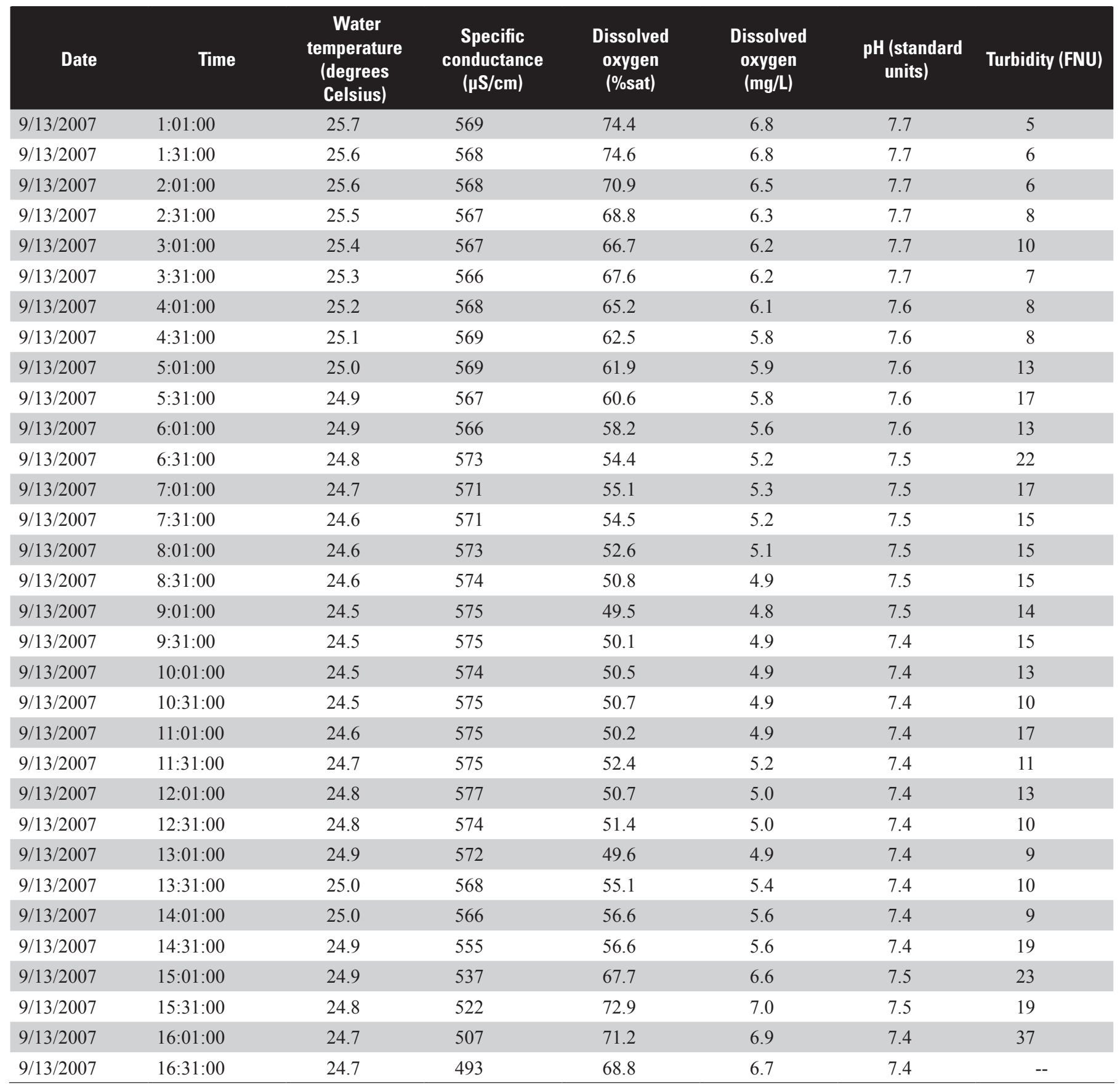


Table 18. Physical property data recorded at 30-minute intervals for station 330730090431300 Murphy Bayou at Murphy, Mississippi, September 10-13, 2007.

$[\mu \mathrm{S} / \mathrm{cm}$, microsiemens per centimeter; \%sat, percent saturation; mg/L, milligrams per liter; FNU, formazin nephelometric units; -- , no data]

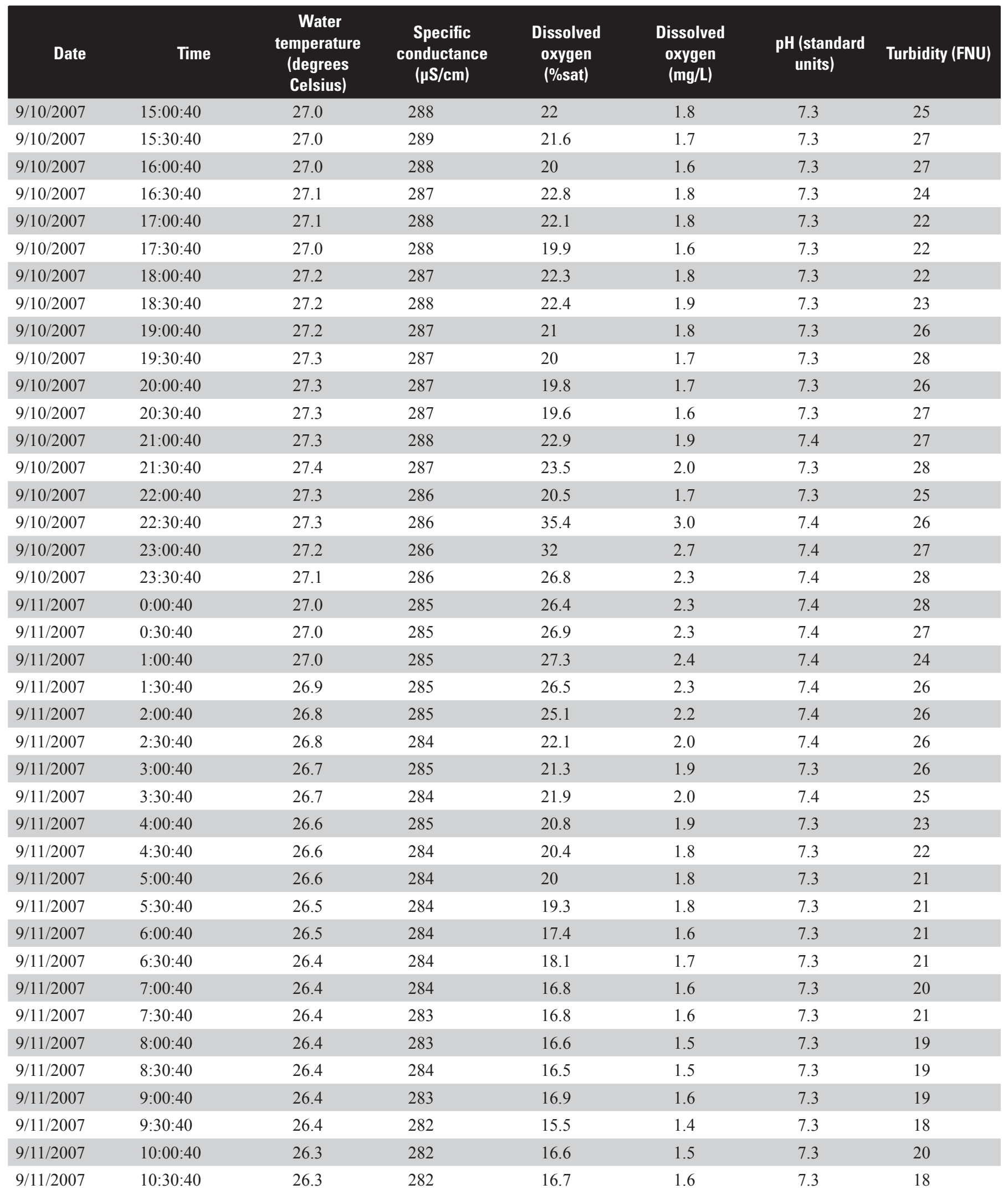


Table 18. Physical property data recorded at 30-minute intervals for station 330730090431300 Murphy Bayou at Murphy, Mississippi, September 10-13, 2007.-Continued

$[\mu \mathrm{S} / \mathrm{cm}$, microsiemens per centimeter; \%sat, percent saturation; mg/L, milligrams per liter; FNU, formazin nephelometric units; -- , no data]

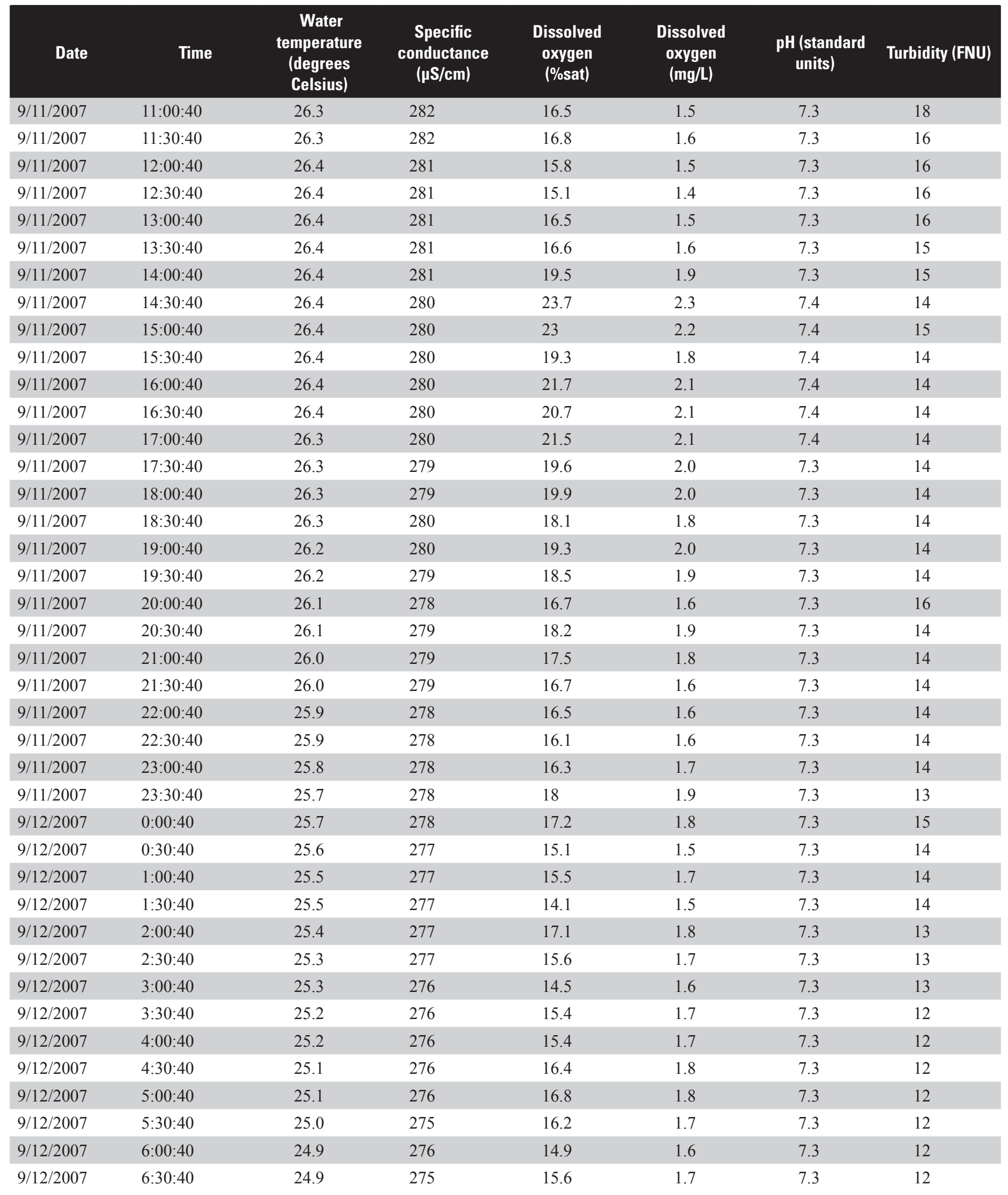


Table 18. Physical property data recorded at 30-minute intervals for station 330730090431300 Murphy Bayou at Murphy, Mississippi, September 10-13, 2007.-Continued

$[\mu \mathrm{S} / \mathrm{cm}$, microsiemens per centimeter; \%sat, percent saturation; mg/L, milligrams per liter; FNU, formazin nephelometric units; -- , no data]

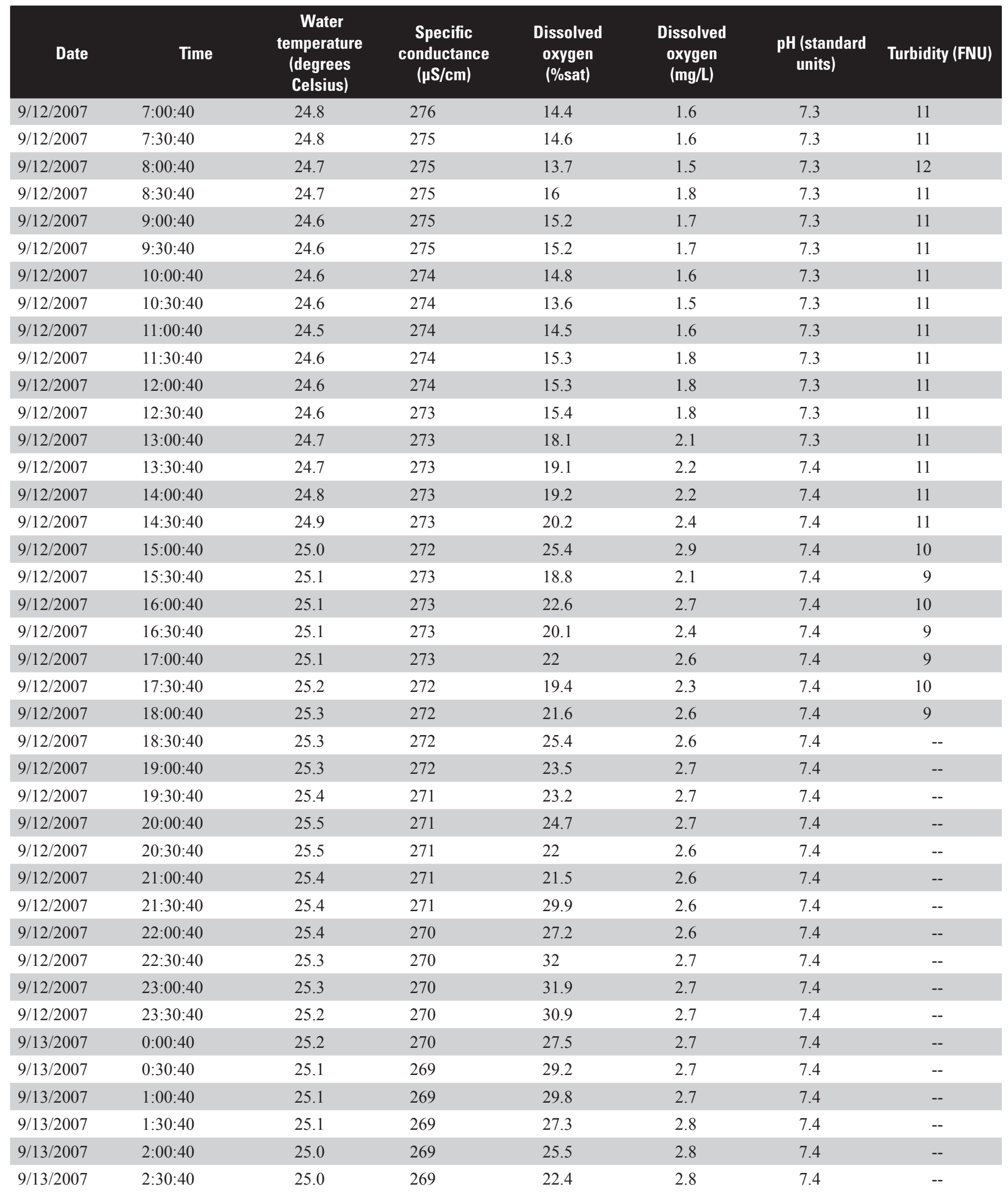


Table 18. Physical property data recorded at 30-minute intervals for station 330730090431300 Murphy Bayou at Murphy, Mississippi, September 10-13, 2007.-Continued

$[\mu \mathrm{S} / \mathrm{cm}$, microsiemens per centimeter; \%sat, percent saturation; mg/L, milligrams per liter; FNU, formazin nephelometric units; -- , no data]

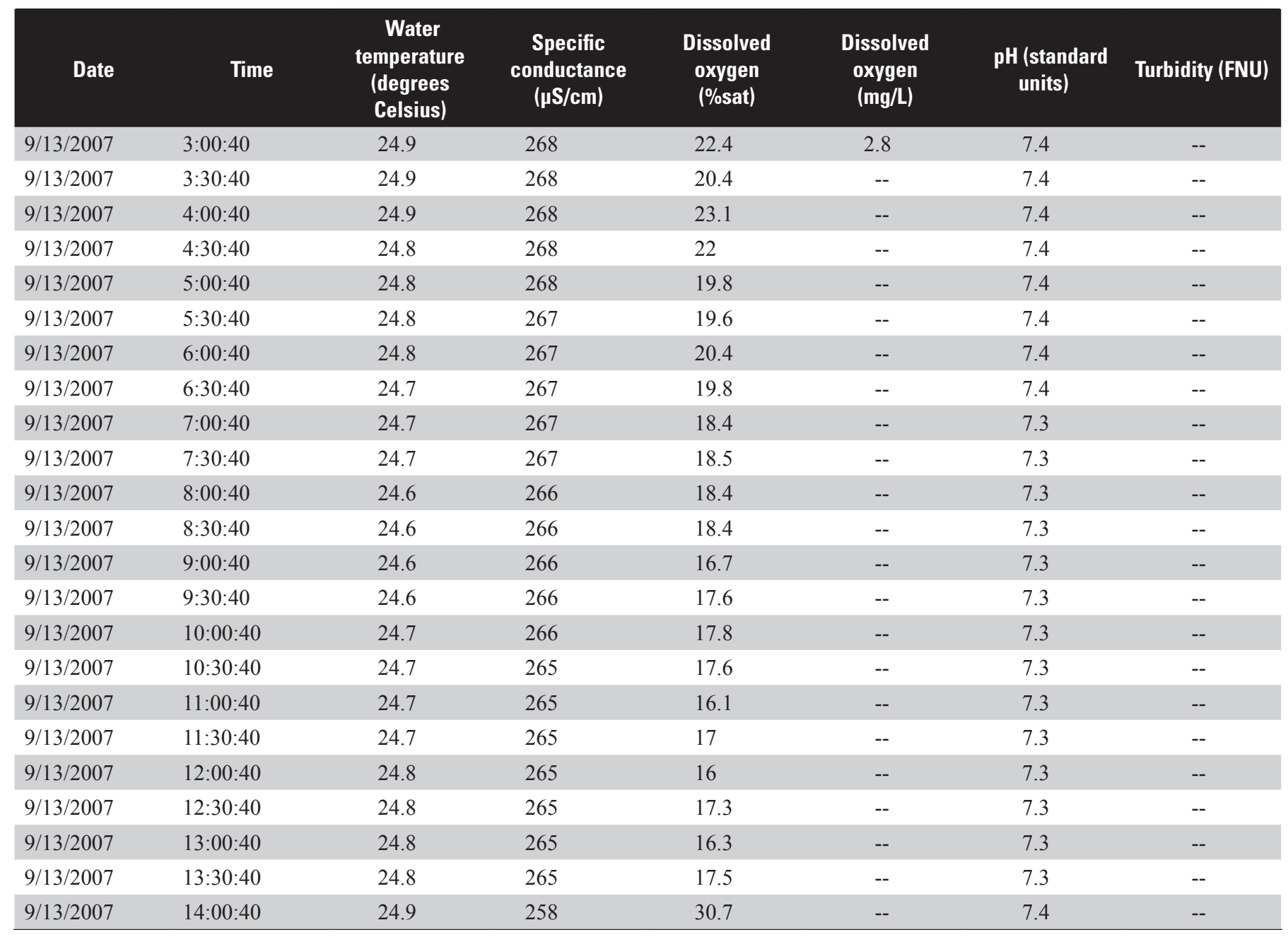


Table 19. Physical property data recorded at 30-minute intervals for station 332749090103400 Pelucia Creek near Rising Sun, Mississippi, October 1-3, 2007.

$[\mu \mathrm{S} / \mathrm{cm}$, microsiemens per centimeter; \%sat, percent saturation; $\mathrm{mg} / \mathrm{L}$, milligrams per liter]

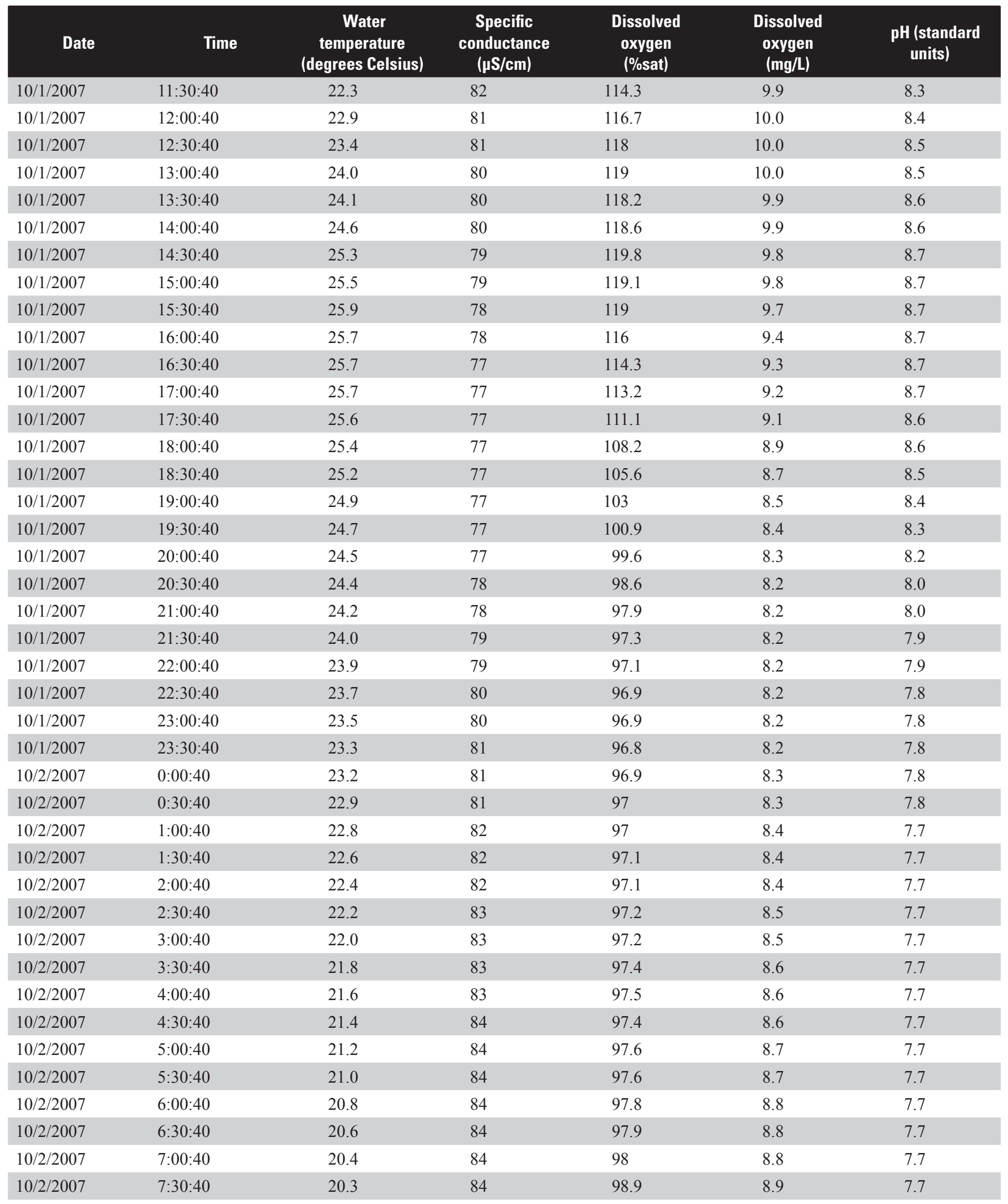


Table 19. Physical property data recorded at 30-minute intervals for station 332749090103400 Pelucia Creek near Rising Sun, Mississippi, October 1-3, 2007.-Continued

$[\mu \mathrm{S} / \mathrm{cm}$, microsiemens per centimeter; \%sat, percent saturation; $\mathrm{mg} / \mathrm{L}$, milligrams per liter $]$

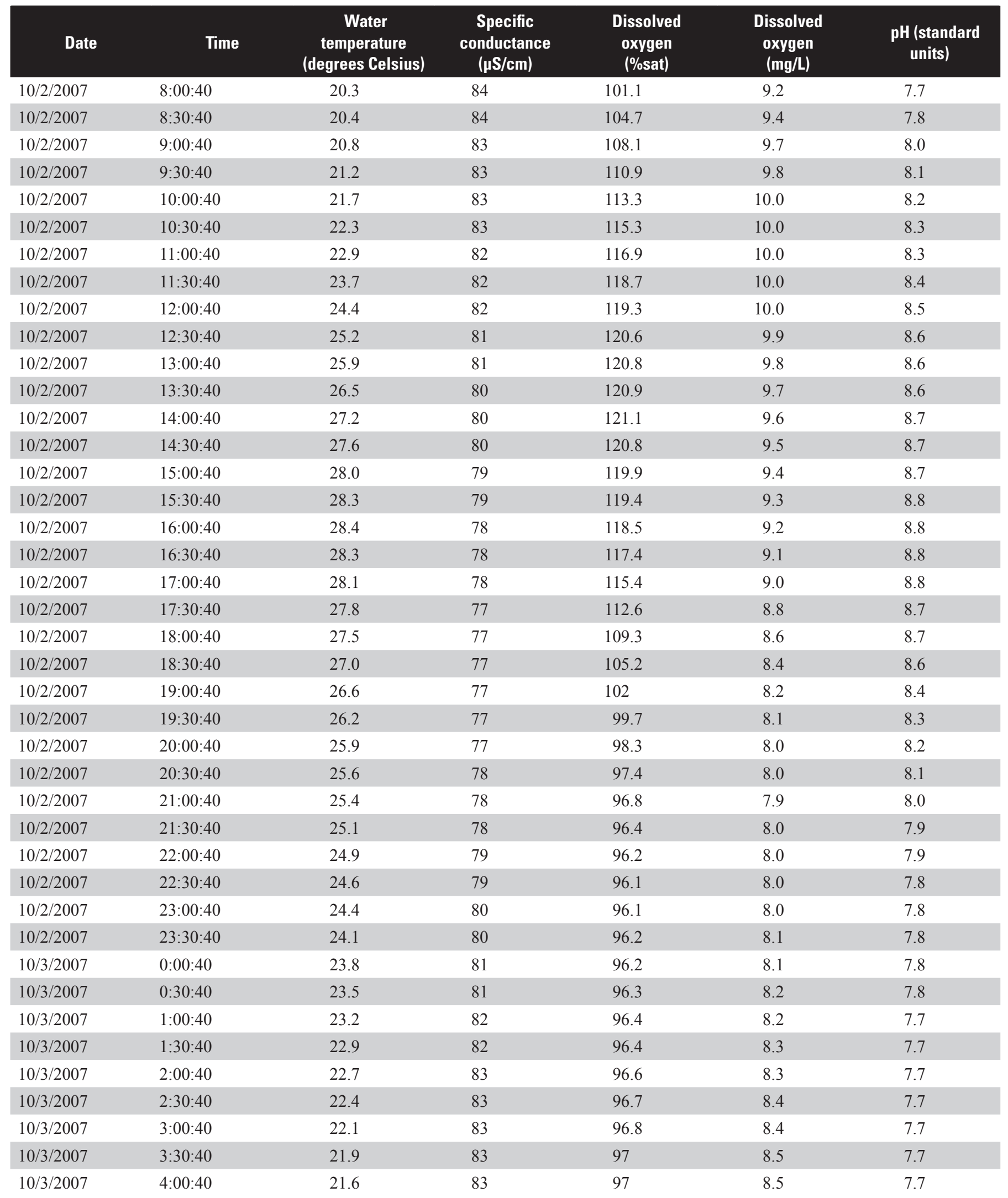


Table 19. Physical property data recorded at 30-minute intervals for station 332749090103400 Pelucia Creek near Rising Sun, Mississippi, October 1-3, 2007.-Continued

$[\mu \mathrm{S} / \mathrm{cm}$, microsiemens per centimeter; \%sat, percent saturation; mg/L, milligrams per liter]

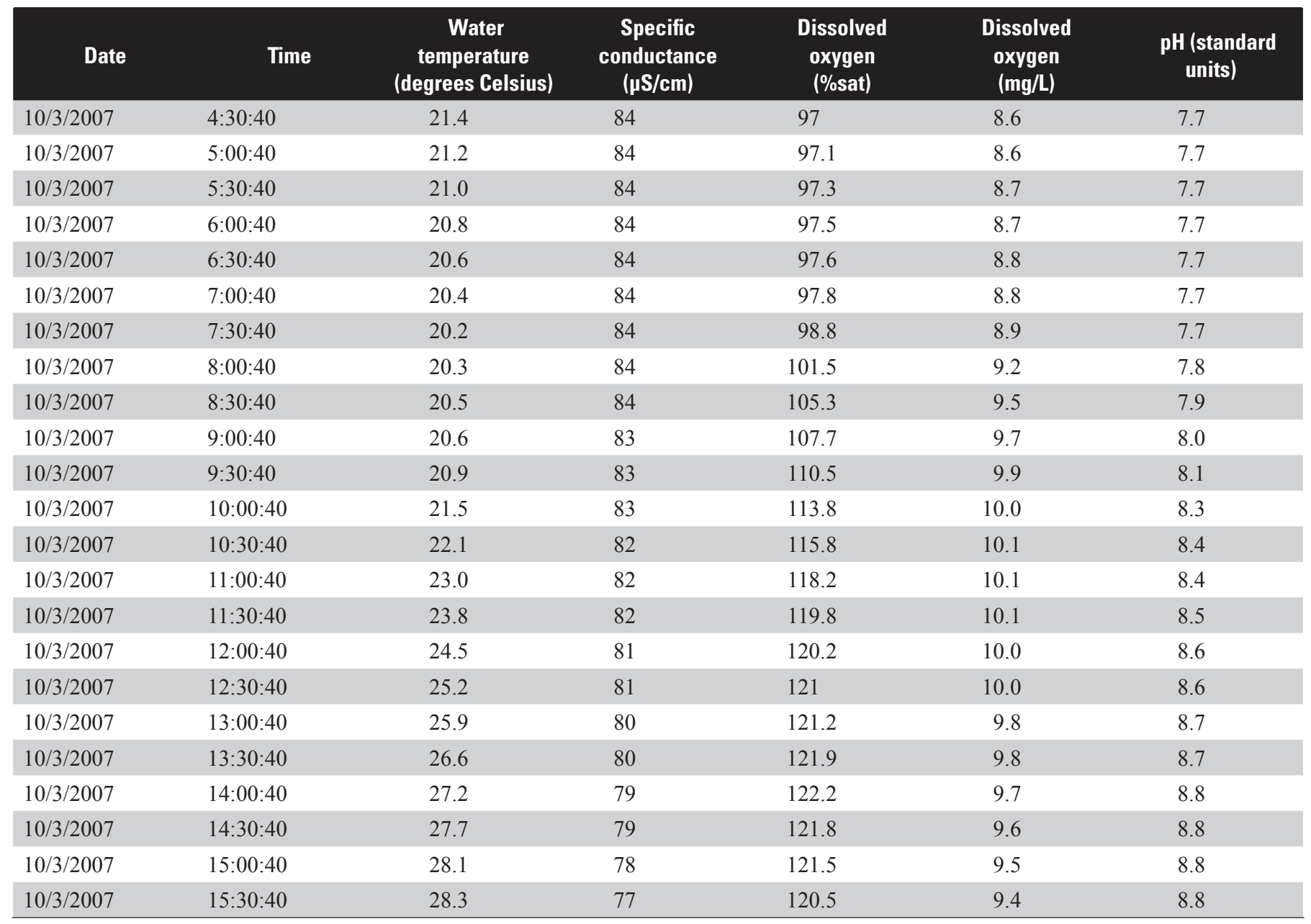


Table 20. Physical property data recorded at 30-minute intervals for station 333328090554800 Clear Creek near Stringtown, Mississippi, September -Continued

$[\mu \mathrm{S} / \mathrm{cm}$, microsiemens per centimeter; \%sat, percent saturation; $\mathrm{mg} / \mathrm{L}$, milligrams per liter $]$

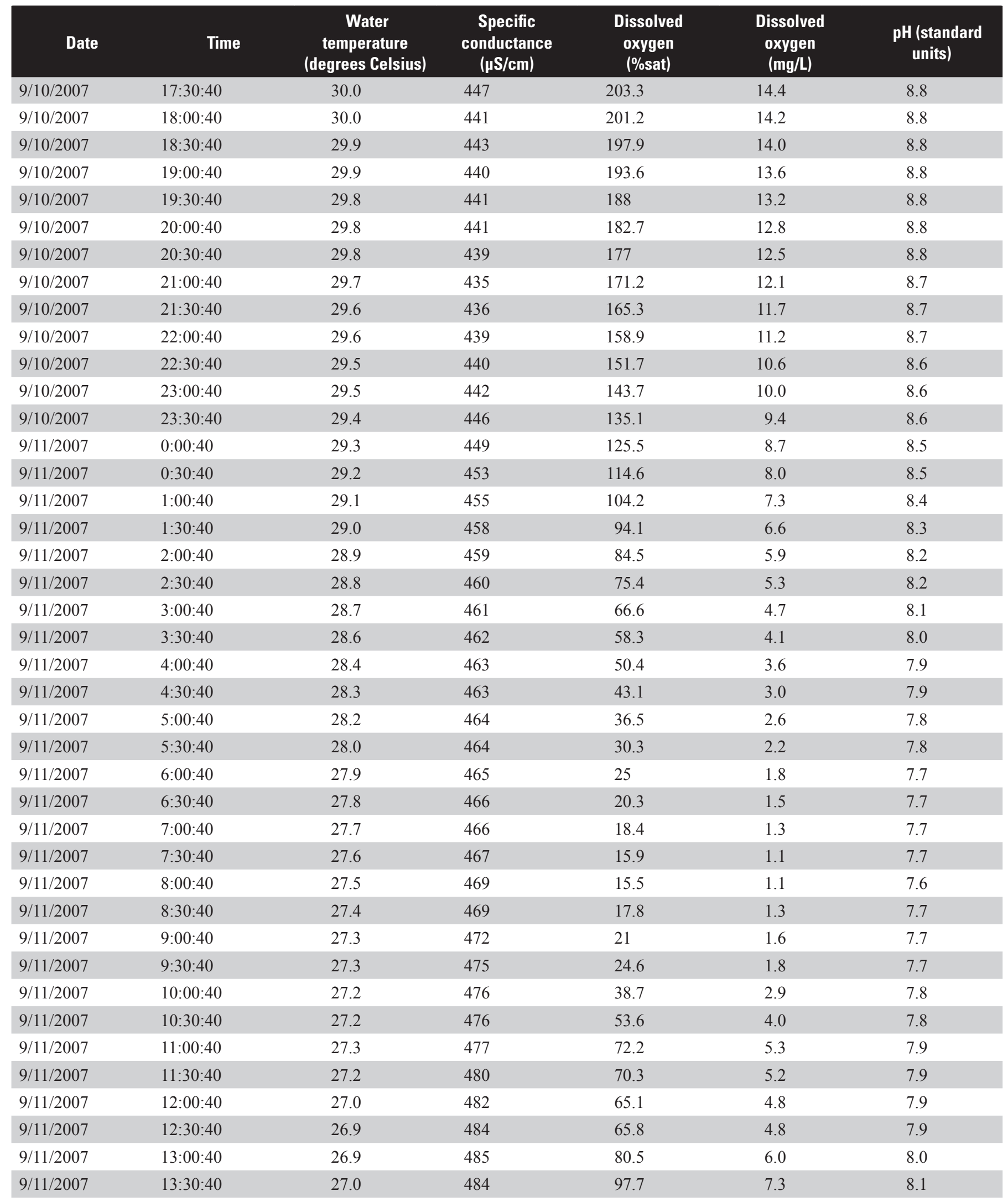


Table 20. Physical property data recorded at 30-minute intervals for station 333328090554800 Clear Creek near Stringtown, Mississippi, September 10-13, 2007.-Continued

$[\mu \mathrm{S} / \mathrm{cm}$, microsiemens per centimeter; \%sat, percent saturation; $\mathrm{mg} / \mathrm{L}$, milligrams per liter]

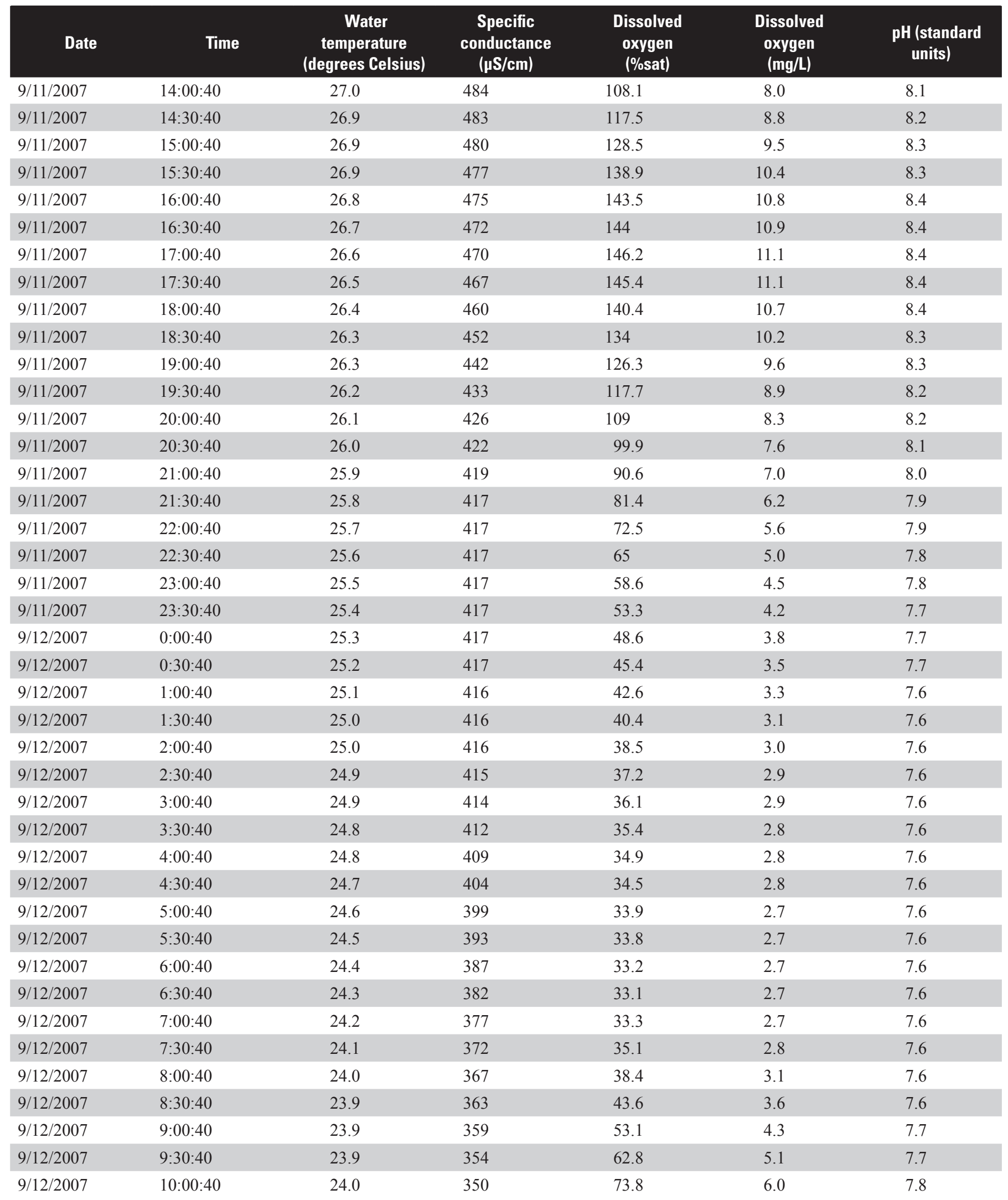


Table 20. Physical property data recorded at 30-minute intervals for station 333328090554800 Clear Creek near Stringtown, Mississippi, September 10-13, 2007.-Continued

$[\mu \mathrm{S} / \mathrm{cm}$, microsiemens per centimeter; \%sat, percent saturation; $\mathrm{mg} / \mathrm{L}$, milligrams per liter $]$

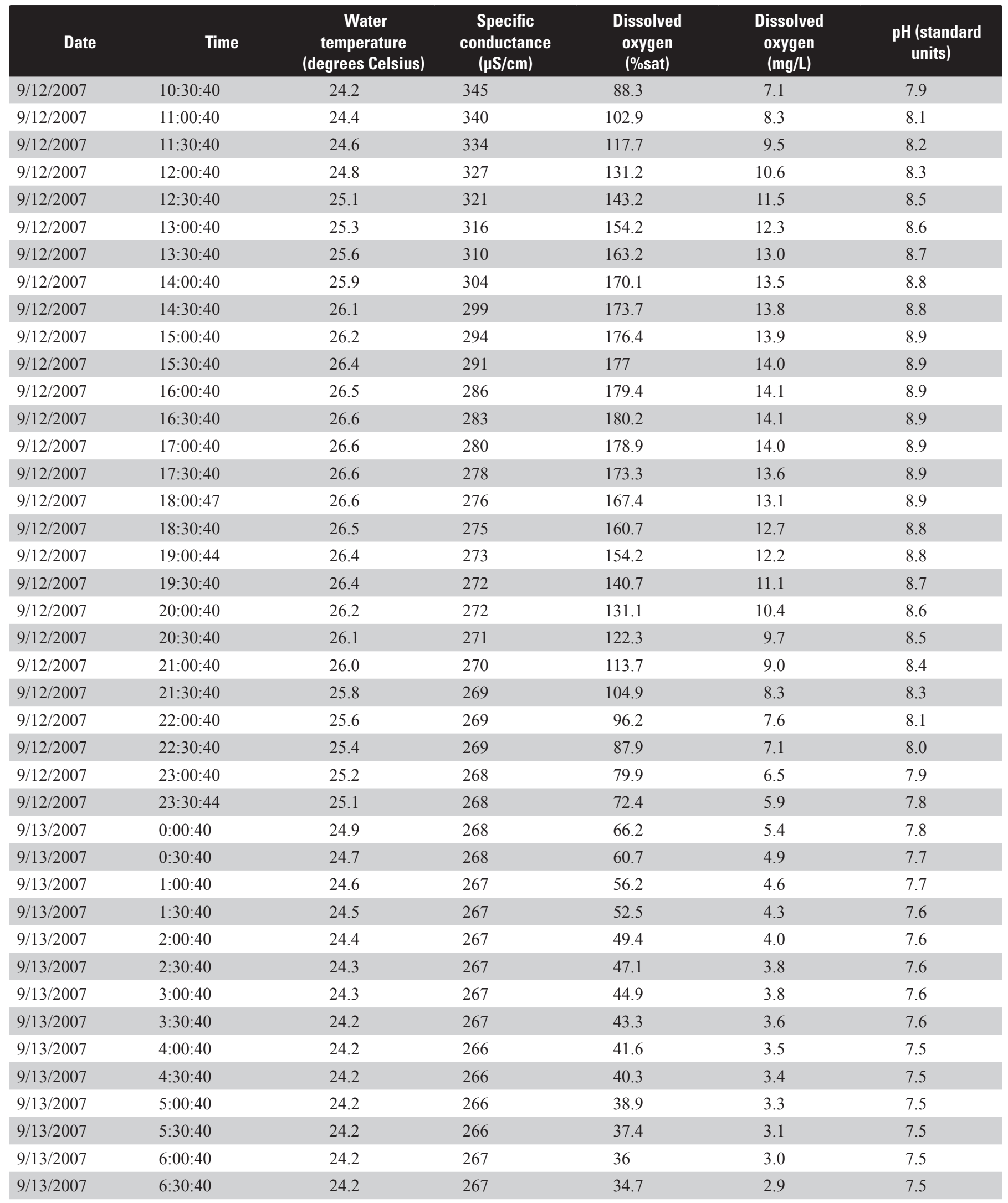


Table 20. Physical property data recorded at 30-minute intervals for station 333328090554800 Clear Creek near Stringtown, Mississippi, September 10-13, 2007.-Continued

$[\mu \mathrm{S} / \mathrm{cm}$, microsiemens per centimeter; \%sat, percent saturation; mg/L, milligrams per liter]

\begin{tabular}{|c|c|c|c|c|c|c|}
\hline Date & Time & $\begin{array}{c}\text { Water } \\
\text { temperature } \\
\text { (degrees Celsius) }\end{array}$ & $\begin{array}{c}\text { Specific } \\
\text { conductance } \\
\text { (pS/cm) }\end{array}$ & $\begin{array}{l}\text { Dissolved } \\
\text { oxygen } \\
\text { (\%sat) }\end{array}$ & $\begin{array}{c}\text { Dissolved } \\
\text { oxygen } \\
\text { (mg/L) }\end{array}$ & $\begin{array}{c}\text { pH (standard } \\
\text { units) }\end{array}$ \\
\hline $9 / 13 / 2007$ & 7:00:40 & 24.1 & 267 & 33.7 & 2.8 & 7.5 \\
\hline $9 / 13 / 2007$ & $7: 30: 40$ & 24.1 & 267 & 33.7 & 2.8 & 7.5 \\
\hline $9 / 13 / 2007$ & $8: 00: 40$ & 24.1 & 267 & 35.1 & 3.0 & 7.5 \\
\hline $9 / 13 / 2007$ & $8: 30: 40$ & 24.1 & 268 & 38.7 & 3.2 & 7.5 \\
\hline $9 / 13 / 2007$ & $9: 30: 40$ & 24.0 & 269 & 46.4 & 3.9 & 7.6 \\
\hline $9 / 13 / 2007$ & $10: 00: 40$ & 24.0 & 269 & 54.1 & 4.5 & 7.6 \\
\hline $9 / 13 / 2007$ & $10: 30: 40$ & 24.0 & 269 & 63.5 & 5.3 & 7.7 \\
\hline $9 / 13 / 2007$ & 11:00:40 & 24.1 & 270 & 72 & 6.0 & 7.8 \\
\hline
\end{tabular}


Table 21. Physical property data recorded at 30-minute intervals for station 333615090061800 Teoc Creek near Teoc, Mississippi, October 1-3, 2007.

$[\mu \mathrm{S} / \mathrm{cm}$, microsiemens per centimeter; \%sat, percent saturation; $\mathrm{mg} / \mathrm{L}$, milligrams per liter]

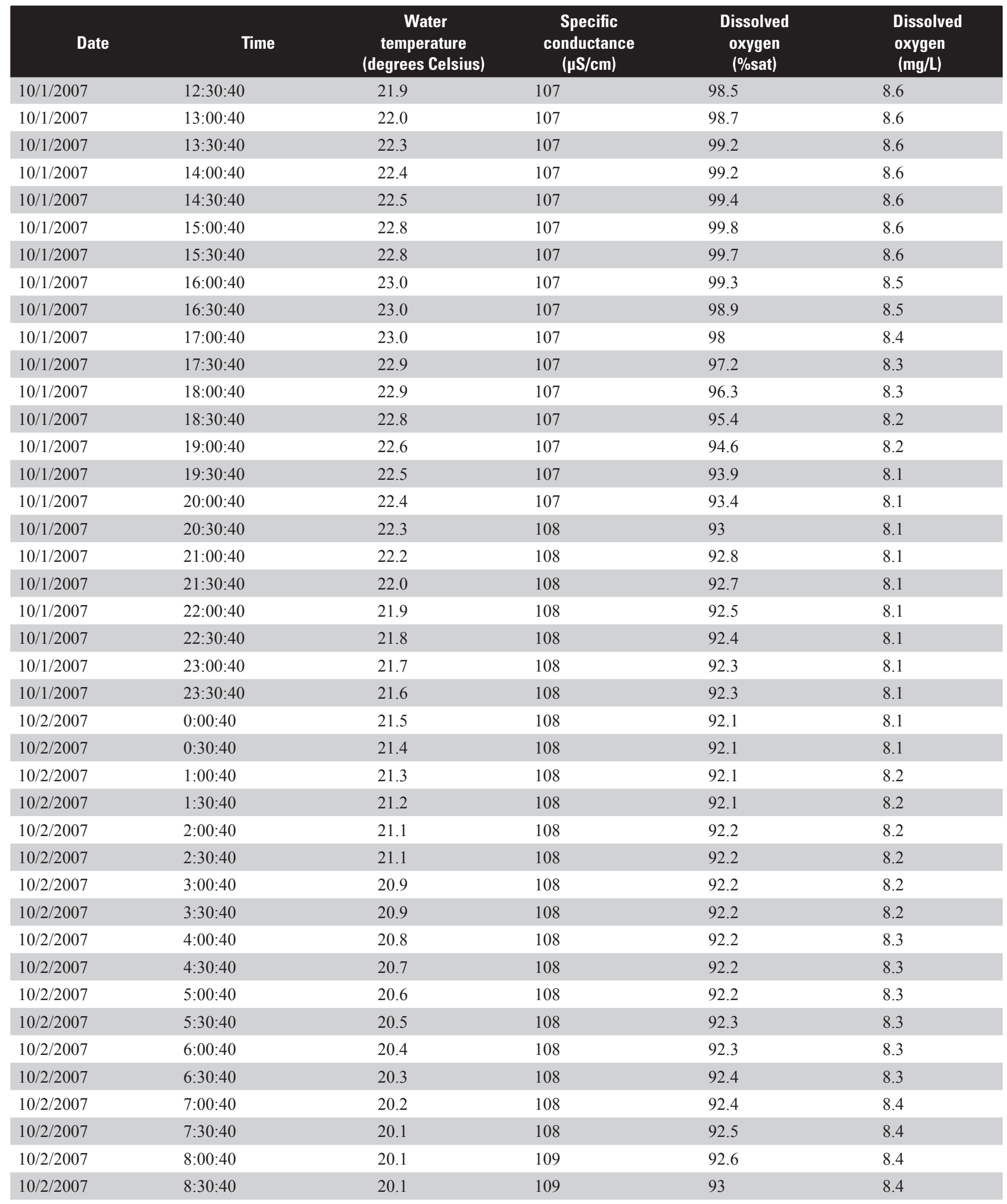


Table 21. Physical property data recorded at 30-minute intervals for station 333615090061800 Teoc Creek near Teoc, Mississippi, October 1-3, 2007.-Continued

$[\mu \mathrm{S} / \mathrm{cm}$, microsiemens per centimeter; \%sat, percent saturation; mg/L, milligrams per liter]

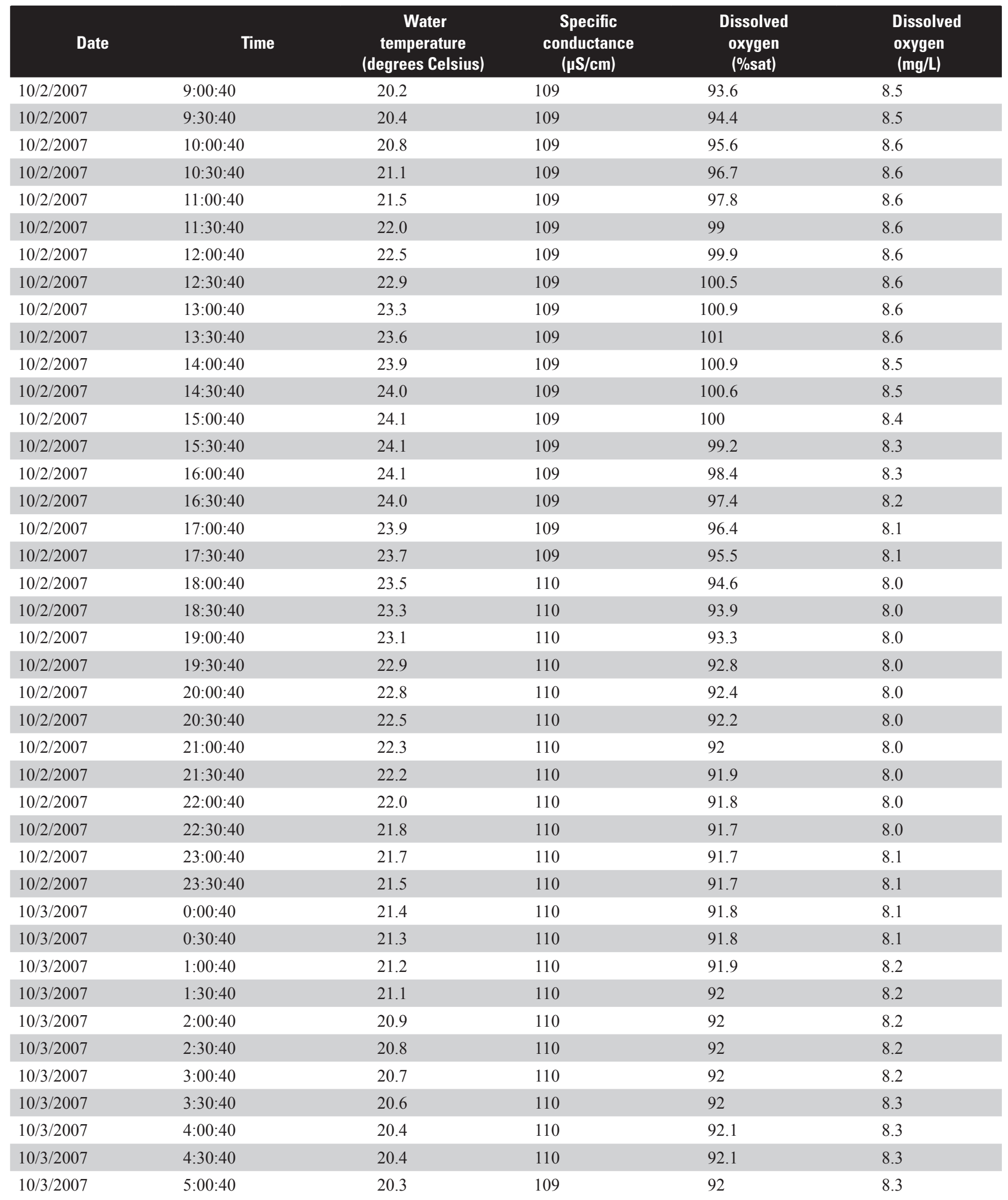


Table 21. Physical property data recorded at 30-minute intervals for station 333615090061800 Teoc Creek near Teoc, Mississippi, October 1-3, 2007.-Continued

$[\mu \mathrm{S} / \mathrm{cm}$, microsiemens per centimeter; \%sat, percent saturation; $\mathrm{mg} / \mathrm{L}$, milligrams per liter]

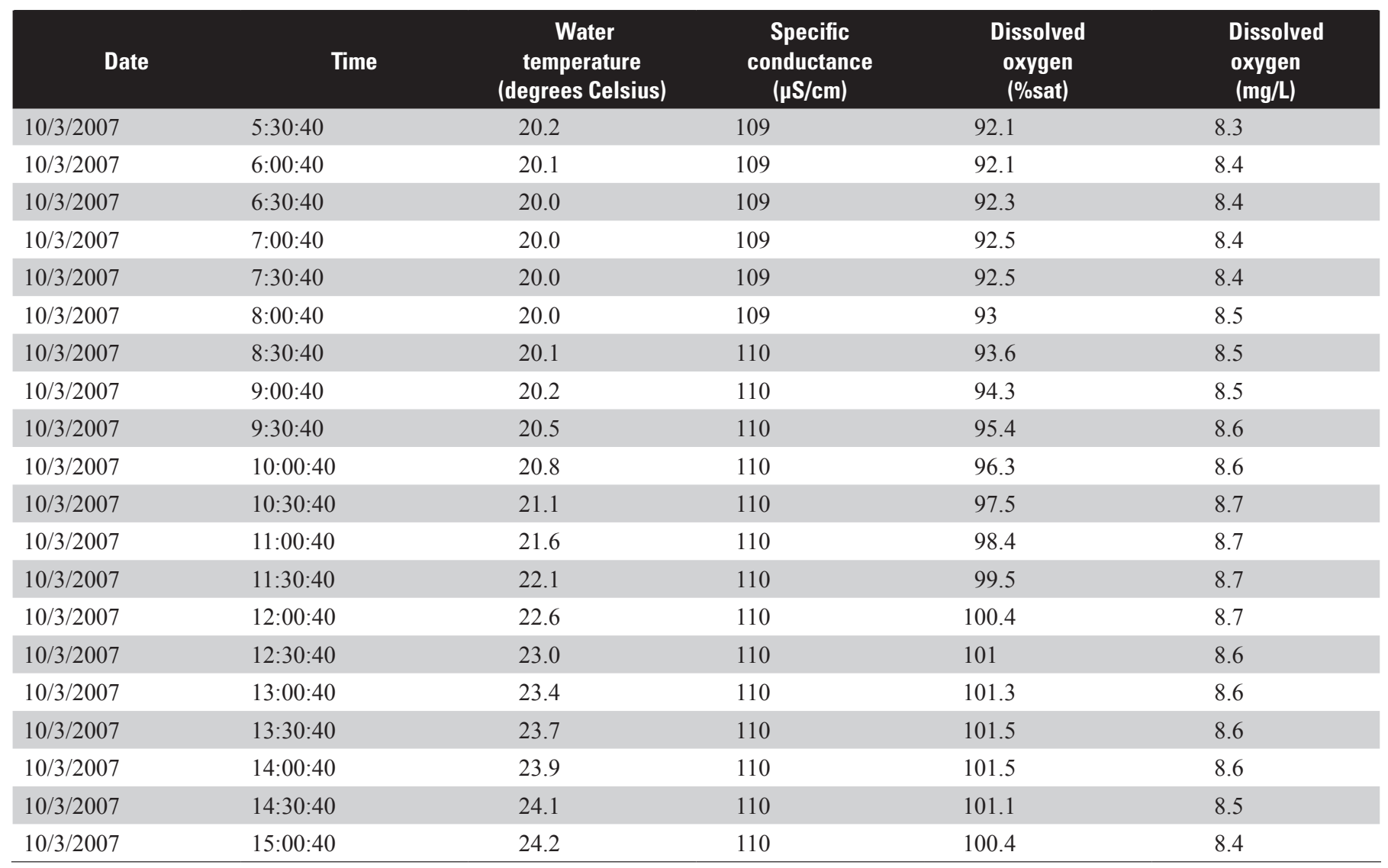


Table 22. Physical property data recorded at 30-minute intervals for station 334747090285000 Wild Bill Bayou near Drew, Mississippi, September 11-13, 2007.

$[\mu \mathrm{S} / \mathrm{cm}$, microsiemens per centimeter; \%sat, percent saturation; $\mathrm{mg} / \mathrm{L}$, milligrams per liter]

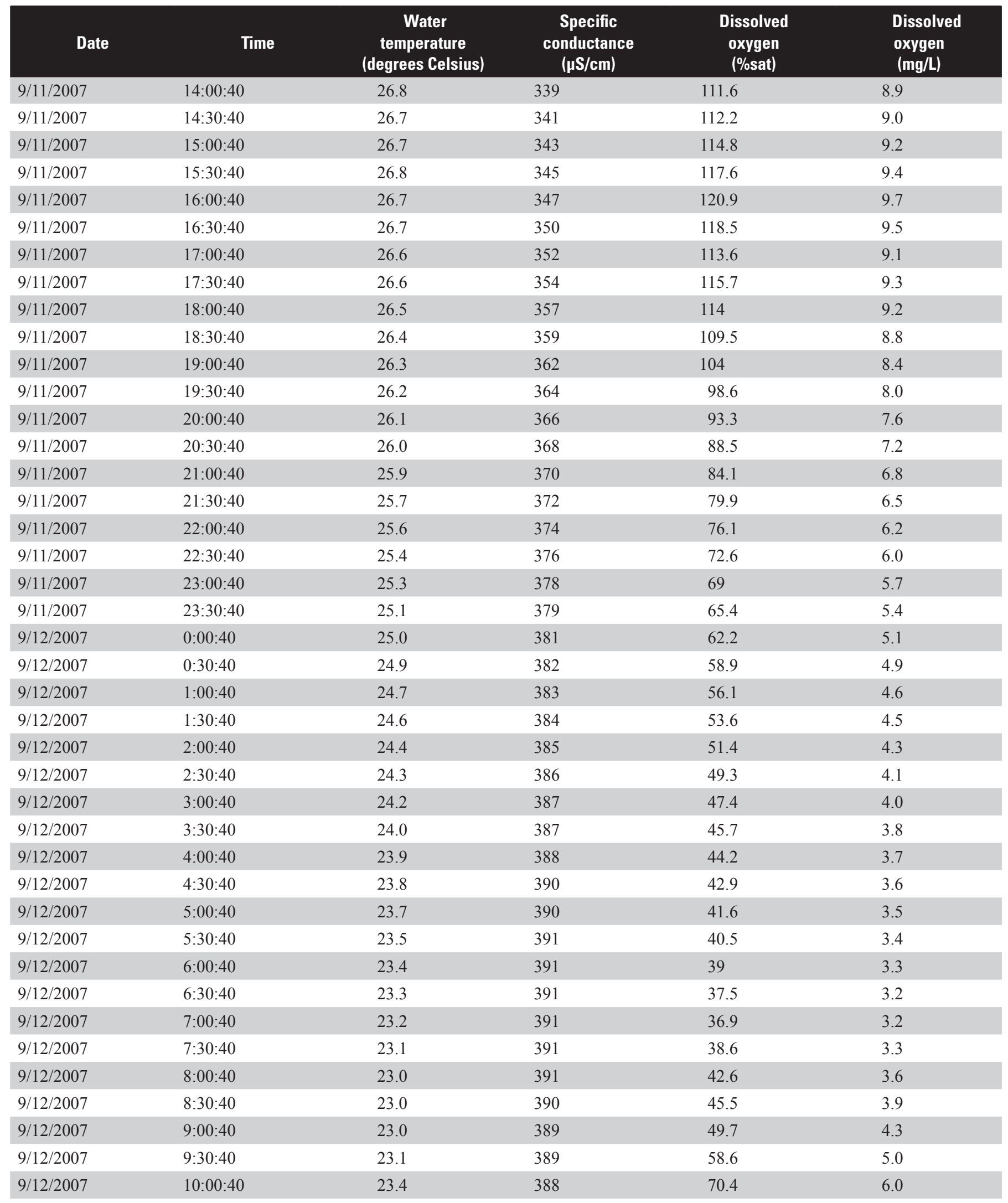


Table 22. Physical property data recorded at 30-minute intervals for station 334747090285000 Wild Bill Bayou near Drew, Mississippi, September 11-13, 2007.-Continued

$[\mu \mathrm{S} / \mathrm{cm}$, microsiemens per centimeter; \%sat, percent saturation; $\mathrm{mg} / \mathrm{L}$, milligrams per liter]

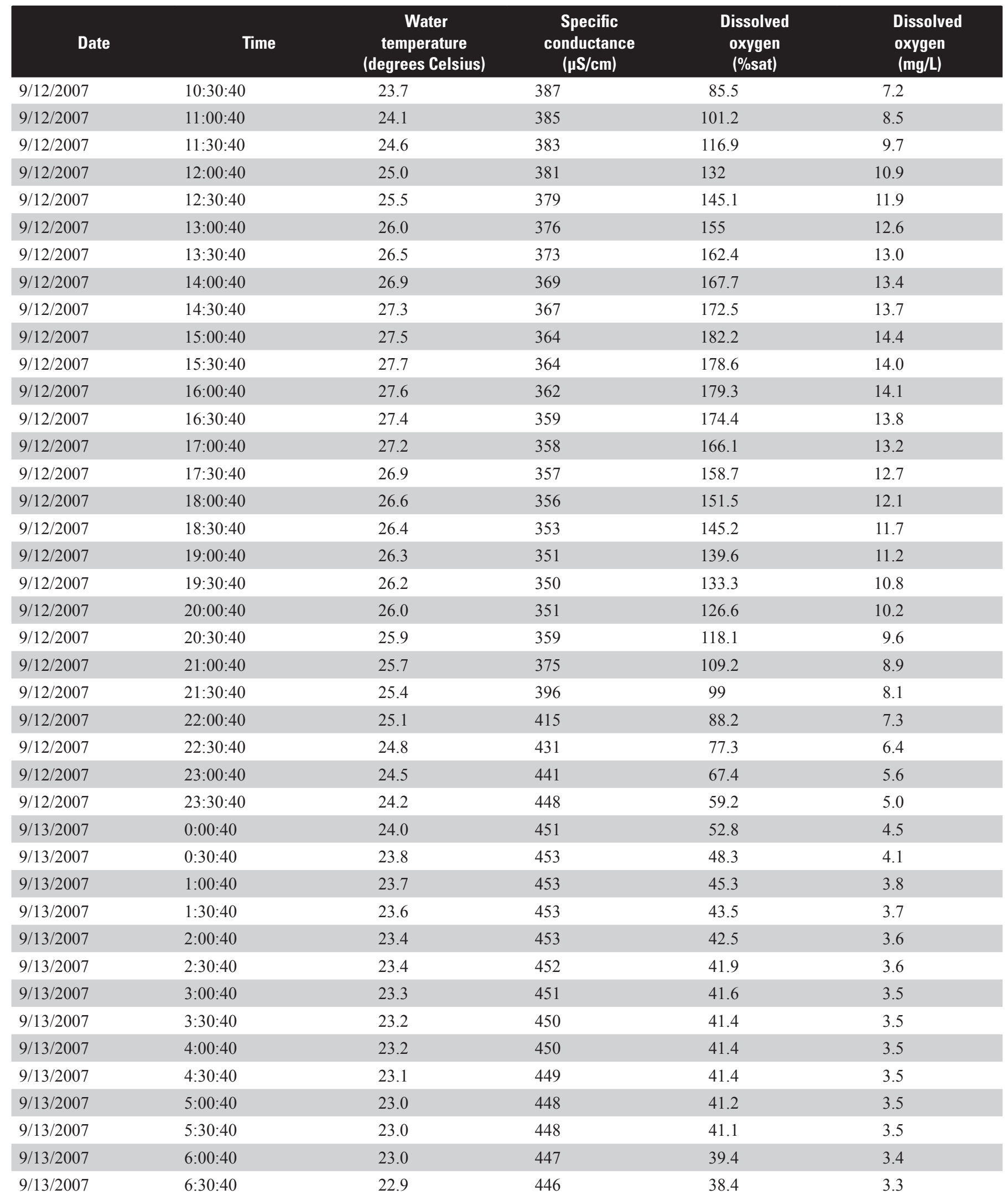


Table 22. Physical property data recorded at 30-minute intervals for station 334747090285000 Wild Bill Bayou near Drew, Mississippi, September 11-13, 2007.-Continued

$[\mu \mathrm{S} / \mathrm{cm}$, microsiemens per centimeter; \%sat, percent saturation; $\mathrm{mg} / \mathrm{L}$, milligrams per liter]

\begin{tabular}{|c|c|c|c|c|c|}
\hline Date & Time & $\begin{array}{c}\text { Water } \\
\text { temperature } \\
\text { (degrees Celsius) }\end{array}$ & $\begin{array}{c}\text { Specific } \\
\text { conductance } \\
\text { (pS/cm) }\end{array}$ & $\begin{array}{l}\text { Dissolved } \\
\text { oxygen } \\
\text { (\%sat) }\end{array}$ & $\begin{array}{l}\text { Dissolved } \\
\text { oxygen } \\
\text { (mg/L) }\end{array}$ \\
\hline $9 / 13 / 2007$ & 7:00:40 & 22.9 & 445 & 38.2 & 3.3 \\
\hline $9 / 13 / 2007$ & $7: 30: 40$ & 22.8 & 445 & 39.4 & 3.4 \\
\hline $9 / 13 / 2007$ & $8: 00: 40$ & 22.8 & 445 & 41.7 & 3.6 \\
\hline $9 / 13 / 2007$ & $8: 30: 40$ & 22.8 & 444 & 45.3 & 3.9 \\
\hline $9 / 13 / 2007$ & 9:30:40 & 22.9 & 443 & 54.3 & 4.7 \\
\hline
\end{tabular}


Table 23. Physical property data recorded at 30-minute intervals for station 335105090415300 Mound Bayou near Merigold, Mississippi, September 24-27, 2007.

$[\mu \mathrm{S} / \mathrm{cm}$, microsiemens per centimeter; \%sat, percent saturation; $\mathrm{mg} / \mathrm{L}$, milligrams per liter $]$

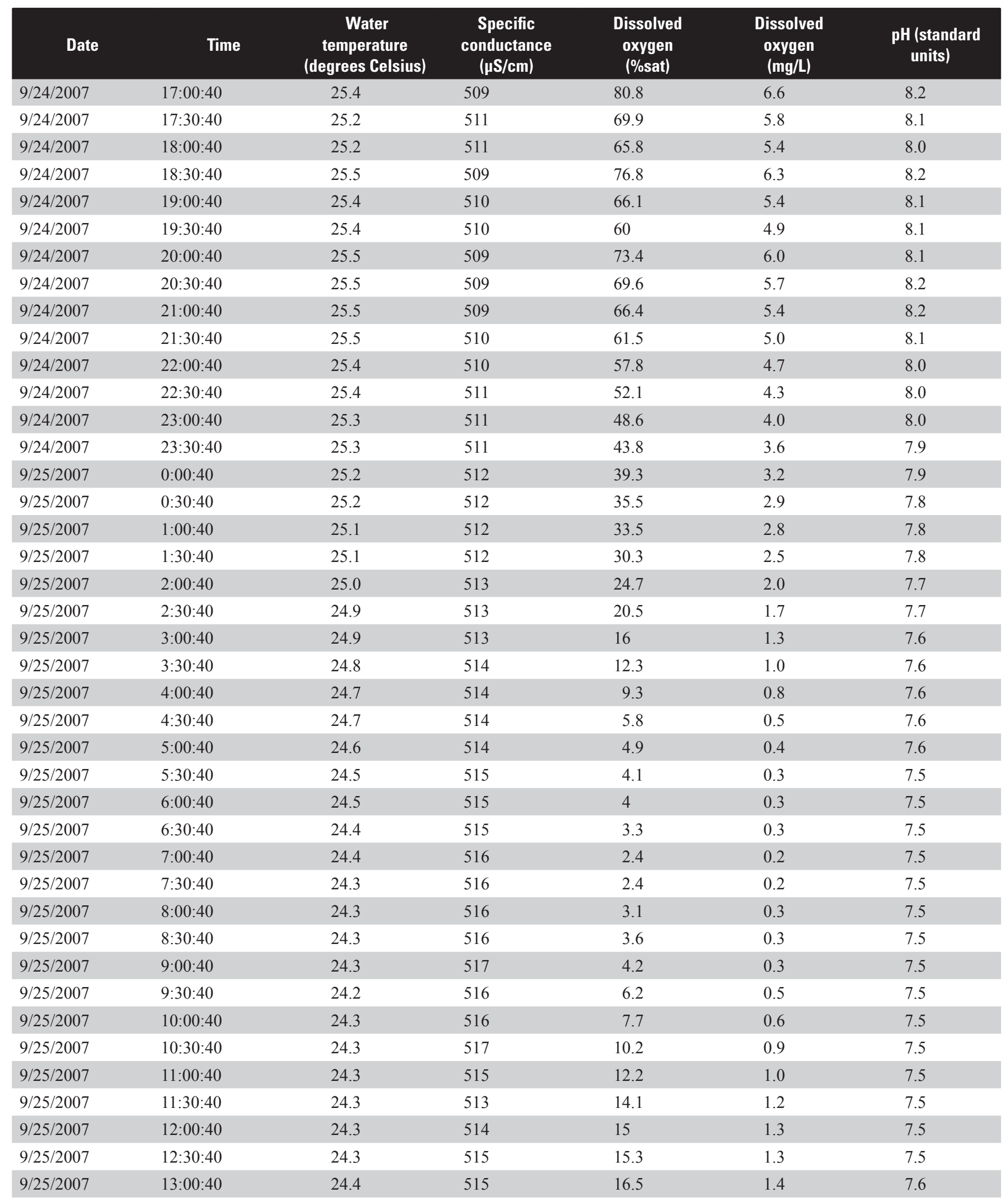


Table 23. Physical property data recorded at 30-minute intervals for station 335105090415300 Mound Bayou near Merigold, Mississippi, September 24-27, 2007.-Continued

$[\mu \mathrm{S} / \mathrm{cm}$, microsiemens per centimeter; \%sat, percent saturation; $\mathrm{mg} / \mathrm{L}$, milligrams per liter]

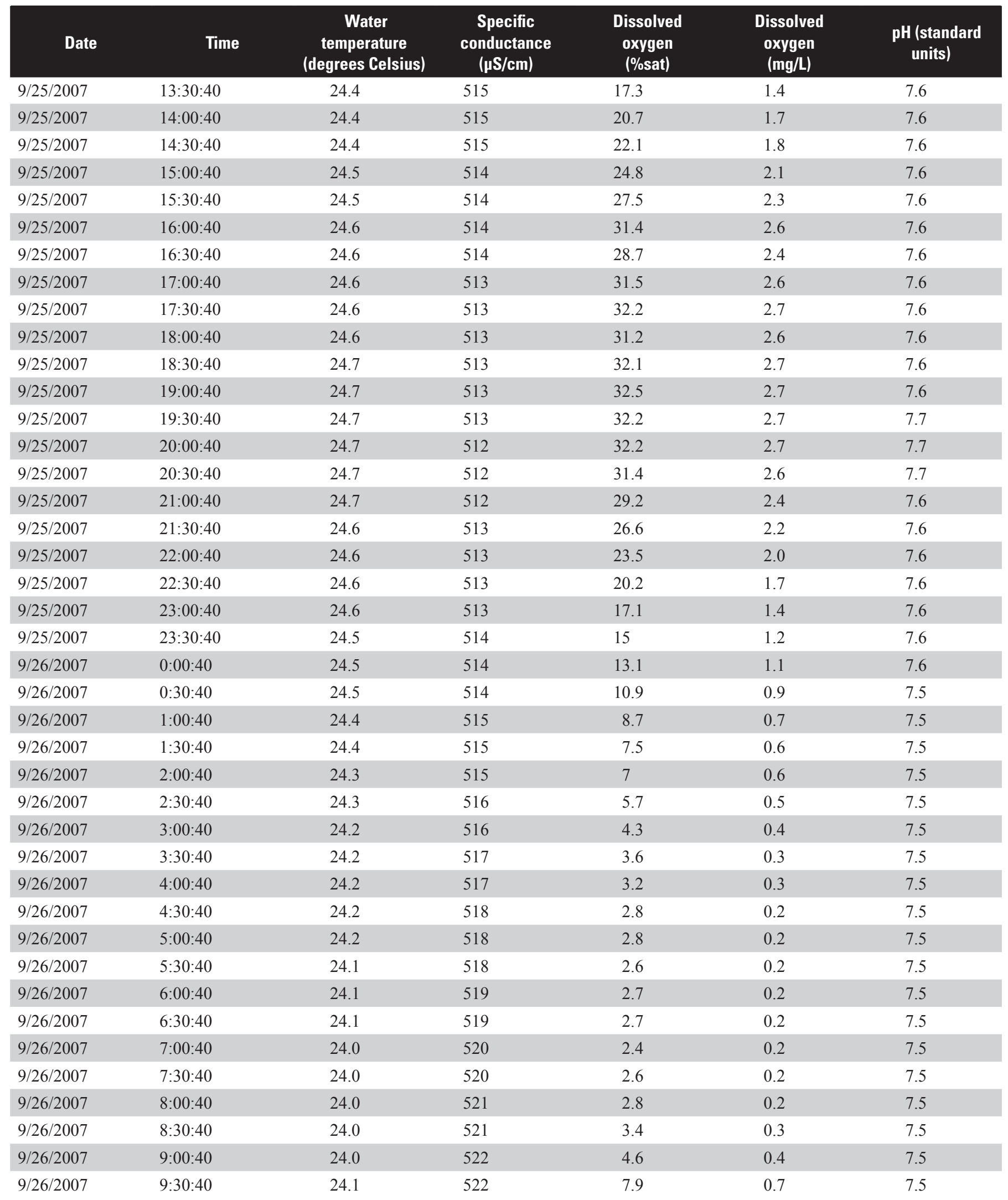


Table 23. Physical property data recorded at 30-minute intervals for station 335105090415300 Mound Bayou near Merigold, Mississippi, September 24-27, 2007.-Continued

$[\mu \mathrm{S} / \mathrm{cm}$, microsiemens per centimeter; \%sat, percent saturation; $\mathrm{mg} / \mathrm{L}$, milligrams per liter $]$

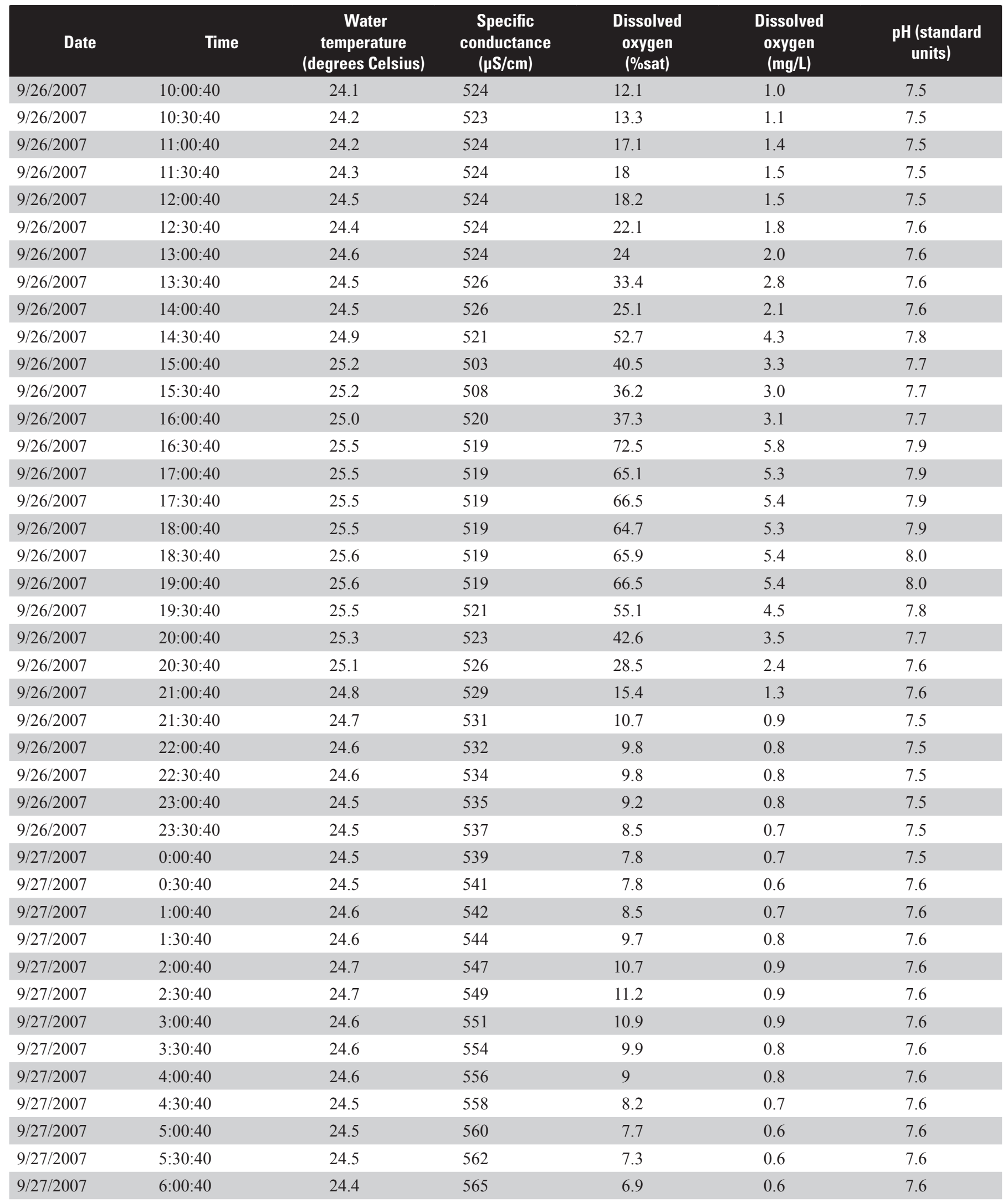


Table 23. Physical property data recorded at 30-minute intervals for station 335105090415300 Mound Bayou near Merigold, Mississippi, September 24-27, 2007.-Continued

$[\mu \mathrm{S} / \mathrm{cm}$, microsiemens per centimeter; \%sat, percent saturation; $\mathrm{mg} / \mathrm{L}$, milligrams per liter]

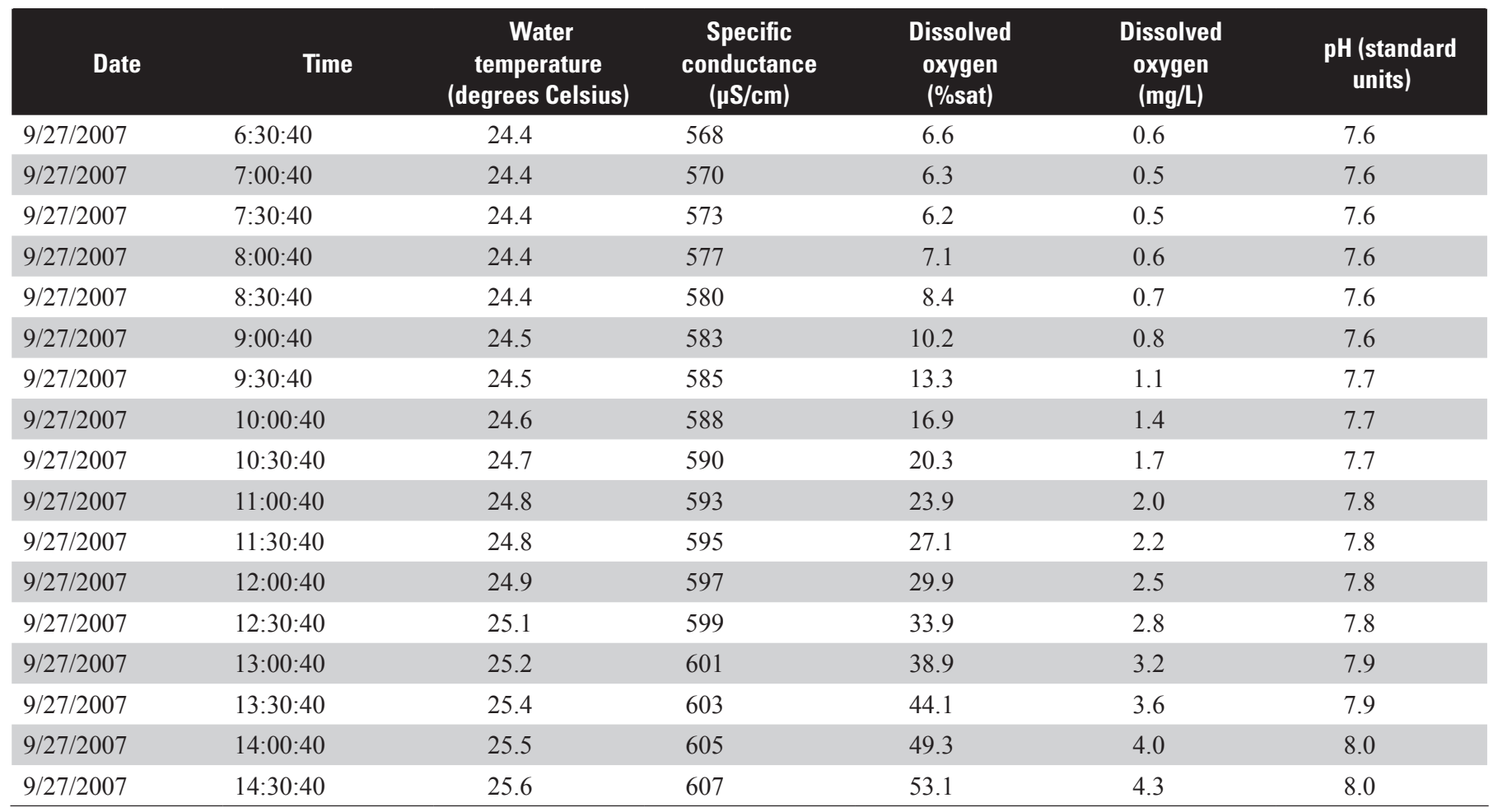


Table 24. Physical property data recorded at 30-minute intervals for station 335458090287000 Bear Bayou near Parchman, Mississippi, September 24-27, 2007.

$[\mu \mathrm{S} / \mathrm{cm}$, microsiemens per centimeter; \%sat, percent saturation; $\mathrm{mg} / \mathrm{L}$, milligrams per liter $]$

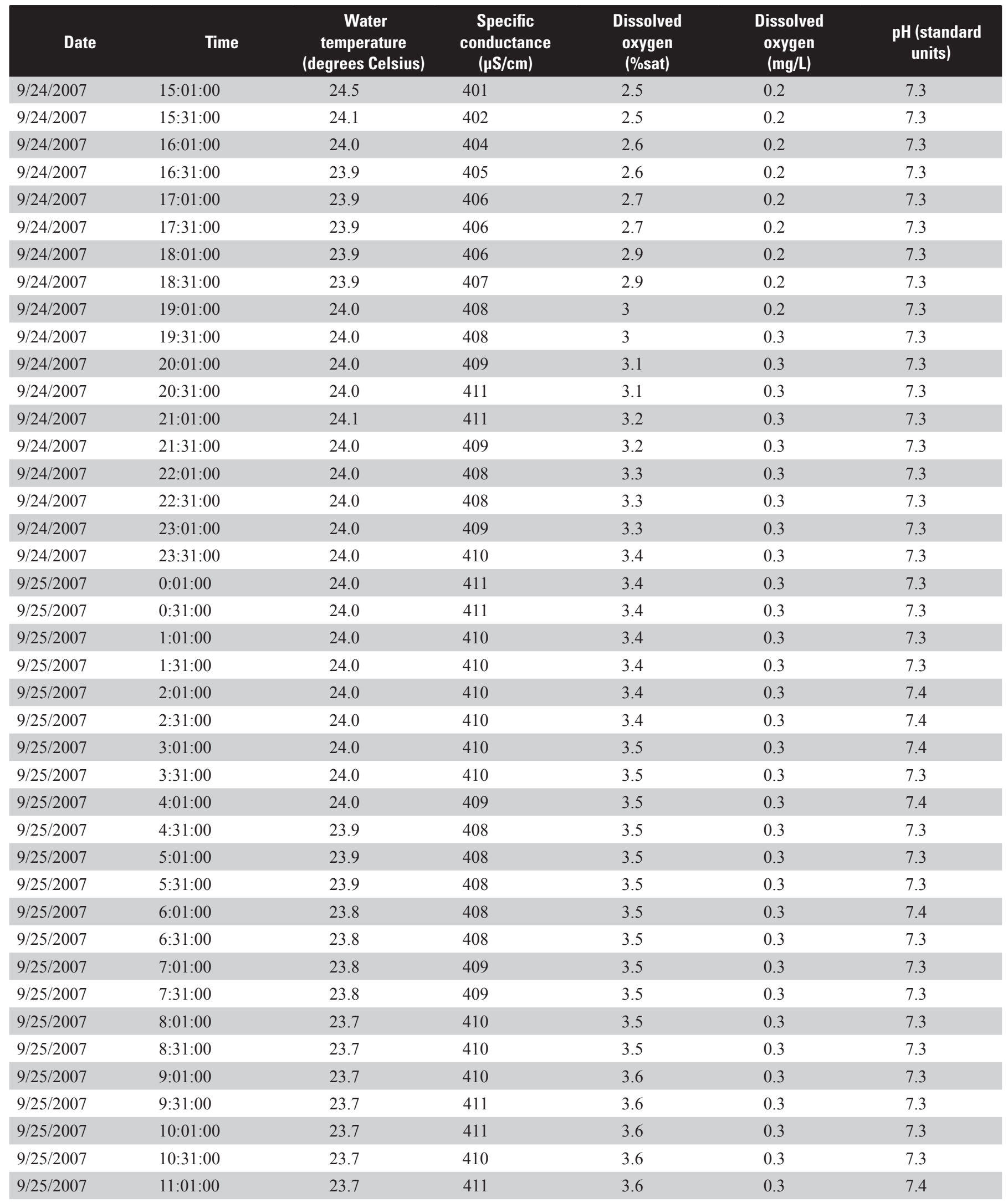


Table 24. Physical property data recorded at 30-minute intervals for station 335458090287000 Bear Bayou near Parchman, Mississippi, September 24-27, 2007.-Continued

$[\mu \mathrm{S} / \mathrm{cm}$, microsiemens per centimeter; \%sat, percent saturation; mg/L, milligrams per liter]

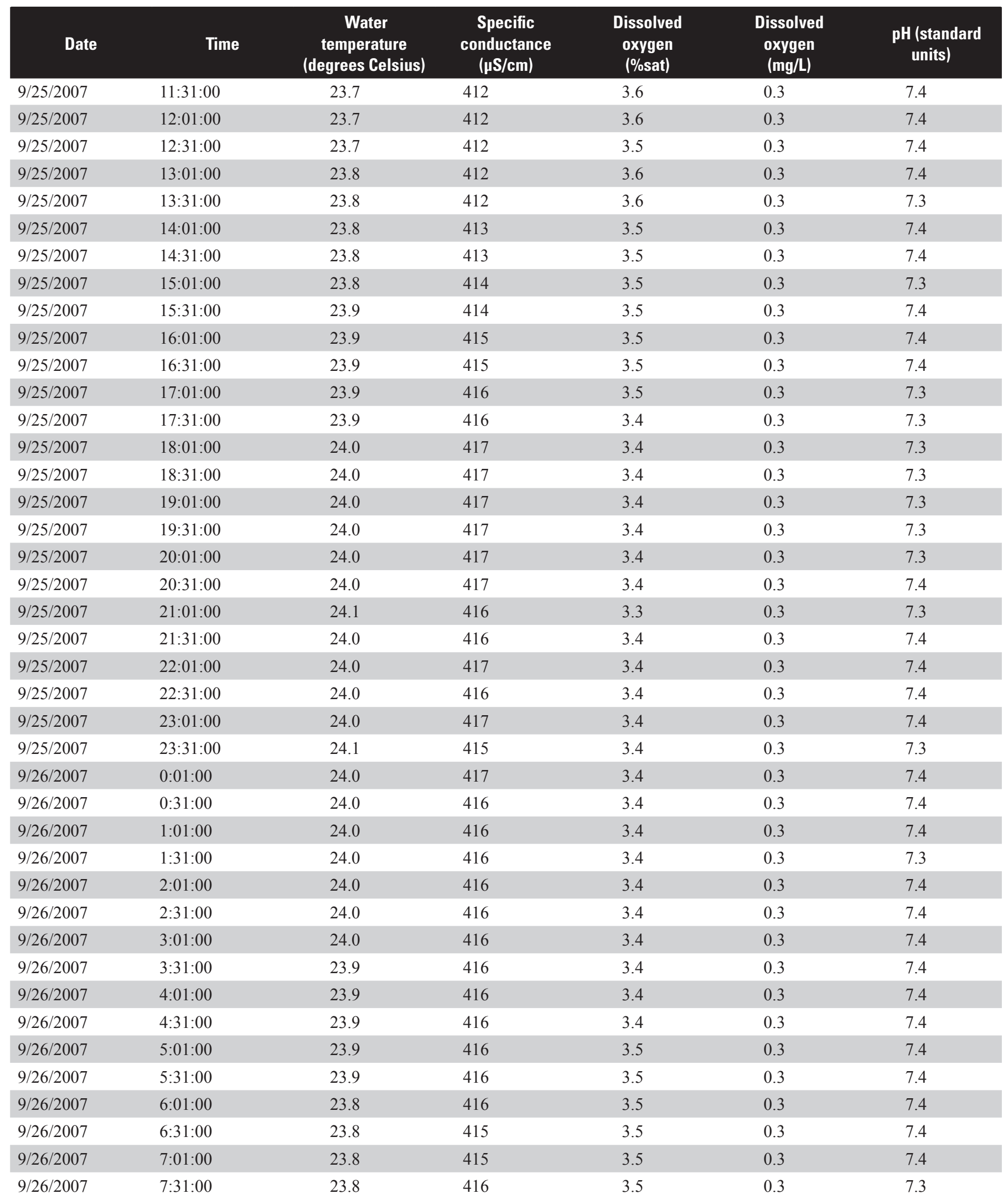


Table 24. Physical property data recorded at 30-minute intervals for station 335458090287000 Bear Bayou near Parchman, Mississippi, September 24-27, 2007.-Continued

$[\mu \mathrm{S} / \mathrm{cm}$, microsiemens per centimeter; \%sat, percent saturation; $\mathrm{mg} / \mathrm{L}$, milligrams per liter $]$

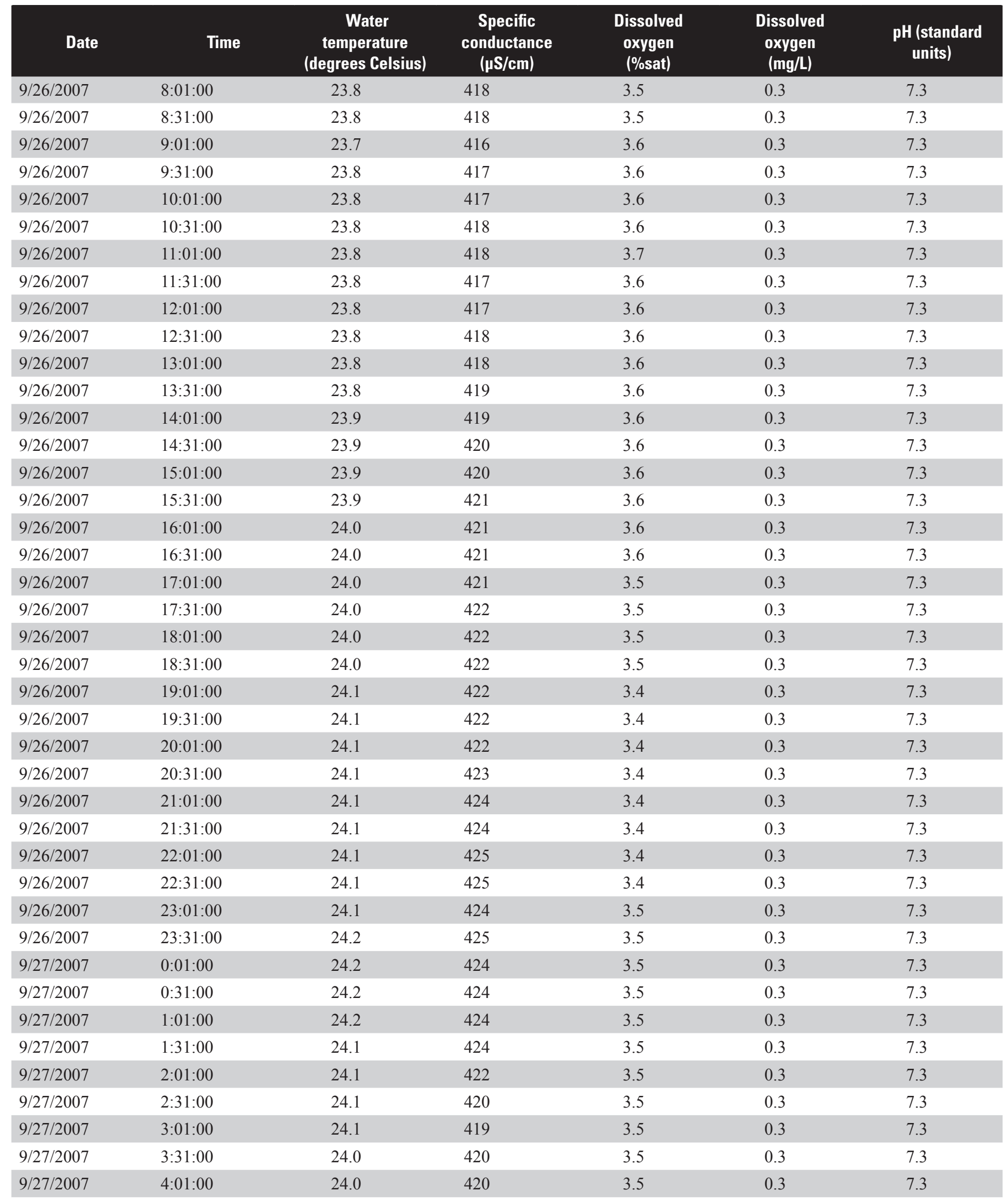


Table 24. Physical property data recorded at 30-minute intervals for station 335458090287000 Bear Bayou near Parchman, Mississippi, September 24-27, 2007.-Continued

$[\mu \mathrm{S} / \mathrm{cm}$, microsiemens per centimeter; \%sat, percent saturation; $\mathrm{mg} / \mathrm{L}$, milligrams per liter]

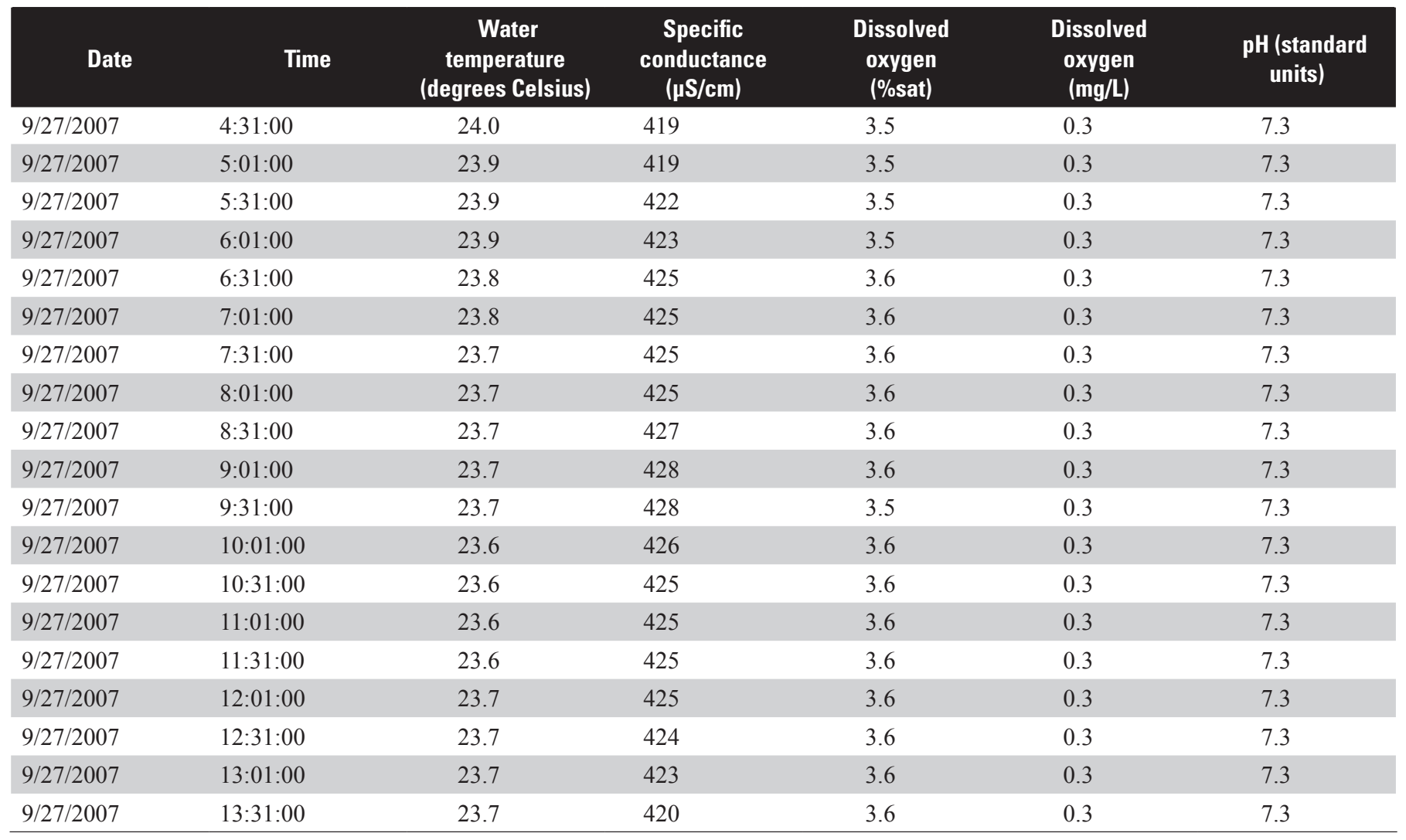


Table 25. Physical property data recorded at 30-minute intervals for station 335749090273300 Home Cypress Bayou near Rome, Mississippi, September 17-20, 2007.

$[\mu \mathrm{S} / \mathrm{cm}$, microsiemens per centimeter; \%sat, percent saturation; $\mathrm{mg} / \mathrm{L}$, milligrams per liter]

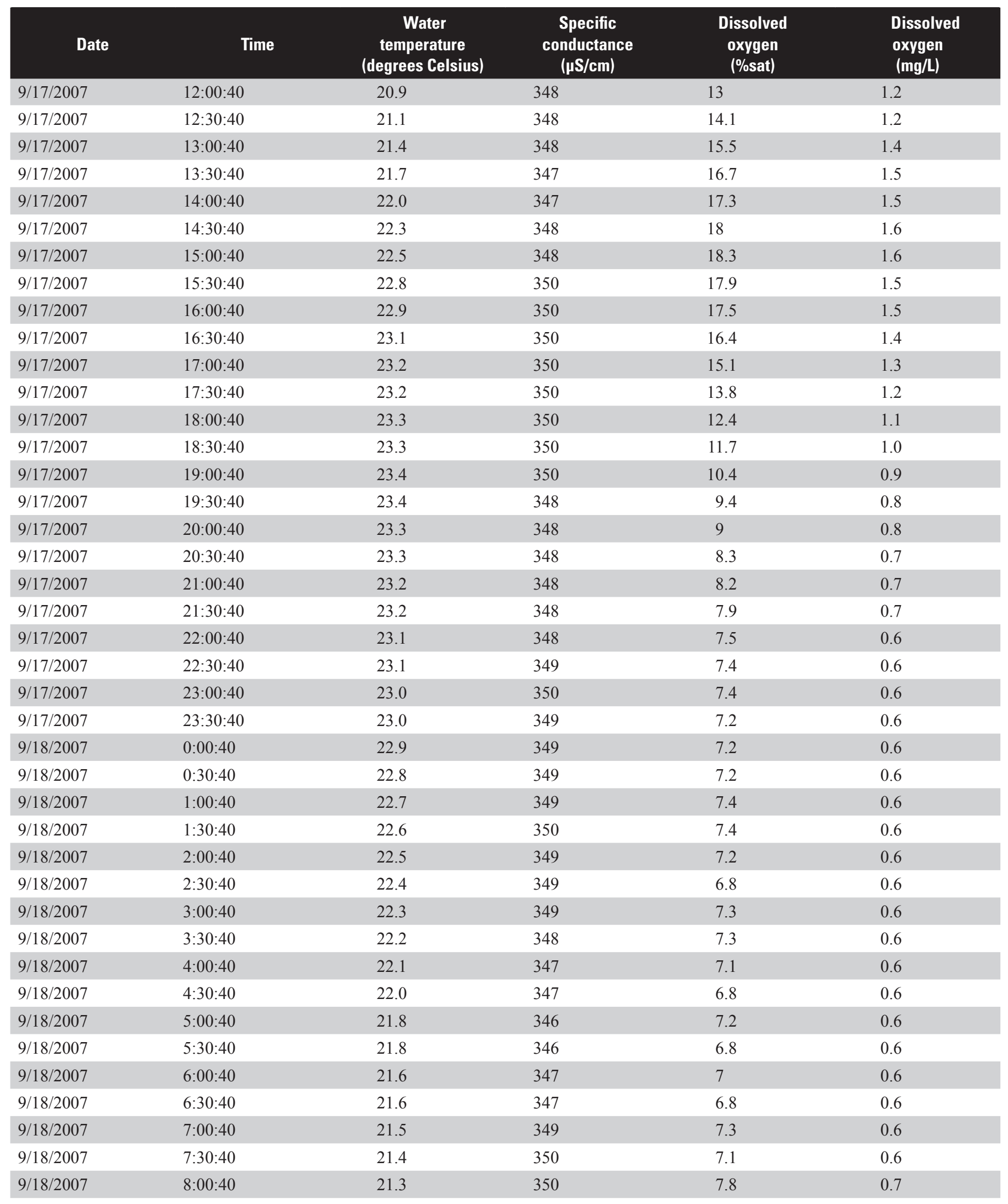


Table 25. Physical property data recorded at 30-minute intervals for station 335749090273300 Home Cypress Bayou near Rome, Mississippi, September 17-20, 2007.-Continued

$[\mu \mathrm{S} / \mathrm{cm}$, microsiemens per centimeter; \%sat, percent saturation; $\mathrm{mg} / \mathrm{L}$, milligrams per liter]

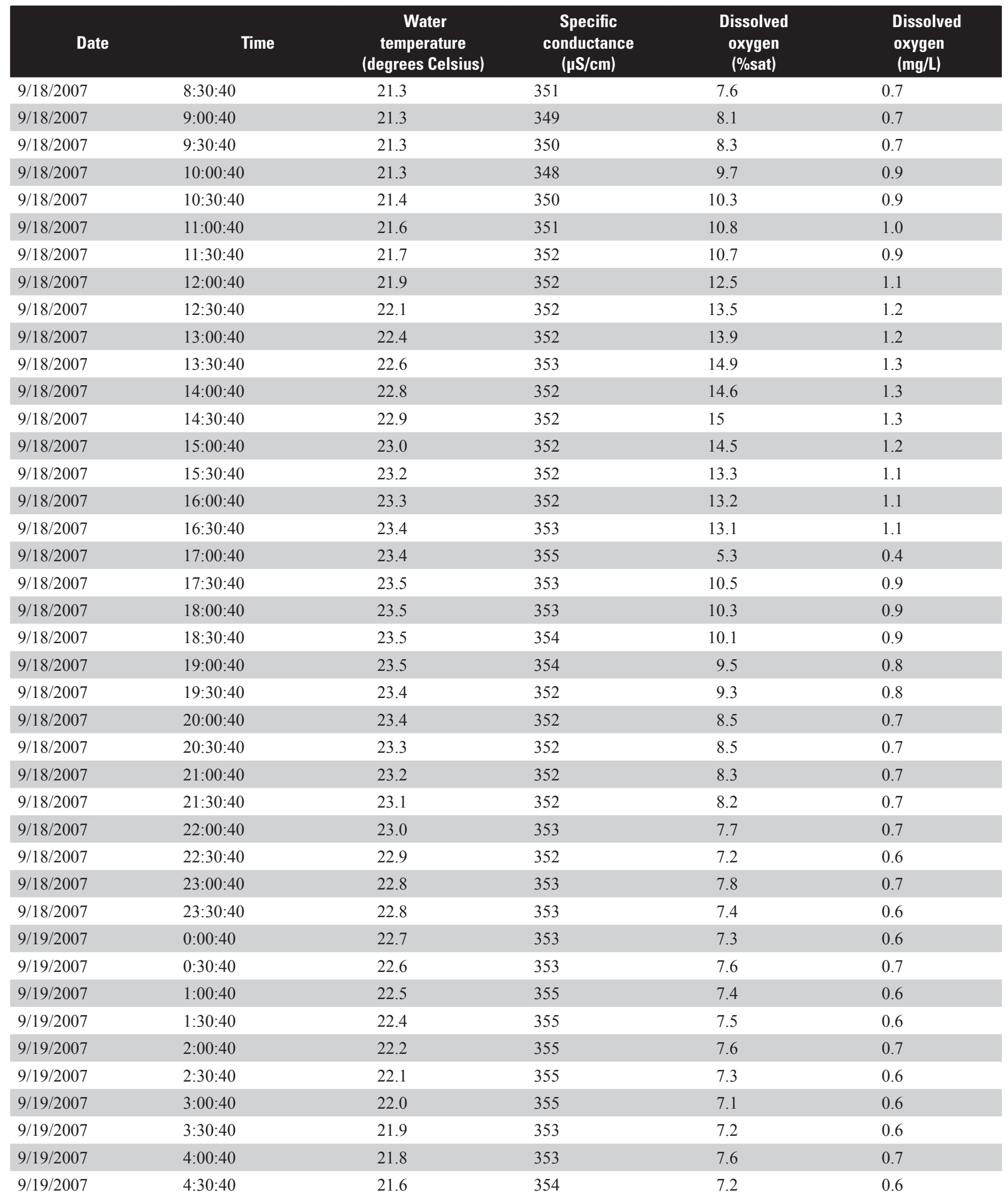


Table 25. Physical property data recorded at 30-minute intervals for station 335749090273300 Home Cypress Bayou near Rome, Mississippi, September 17-20, 2007.-Continued

$[\mu \mathrm{S} / \mathrm{cm}$, microsiemens per centimeter; \%sat, percent saturation; $\mathrm{mg} / \mathrm{L}$, milligrams per liter]

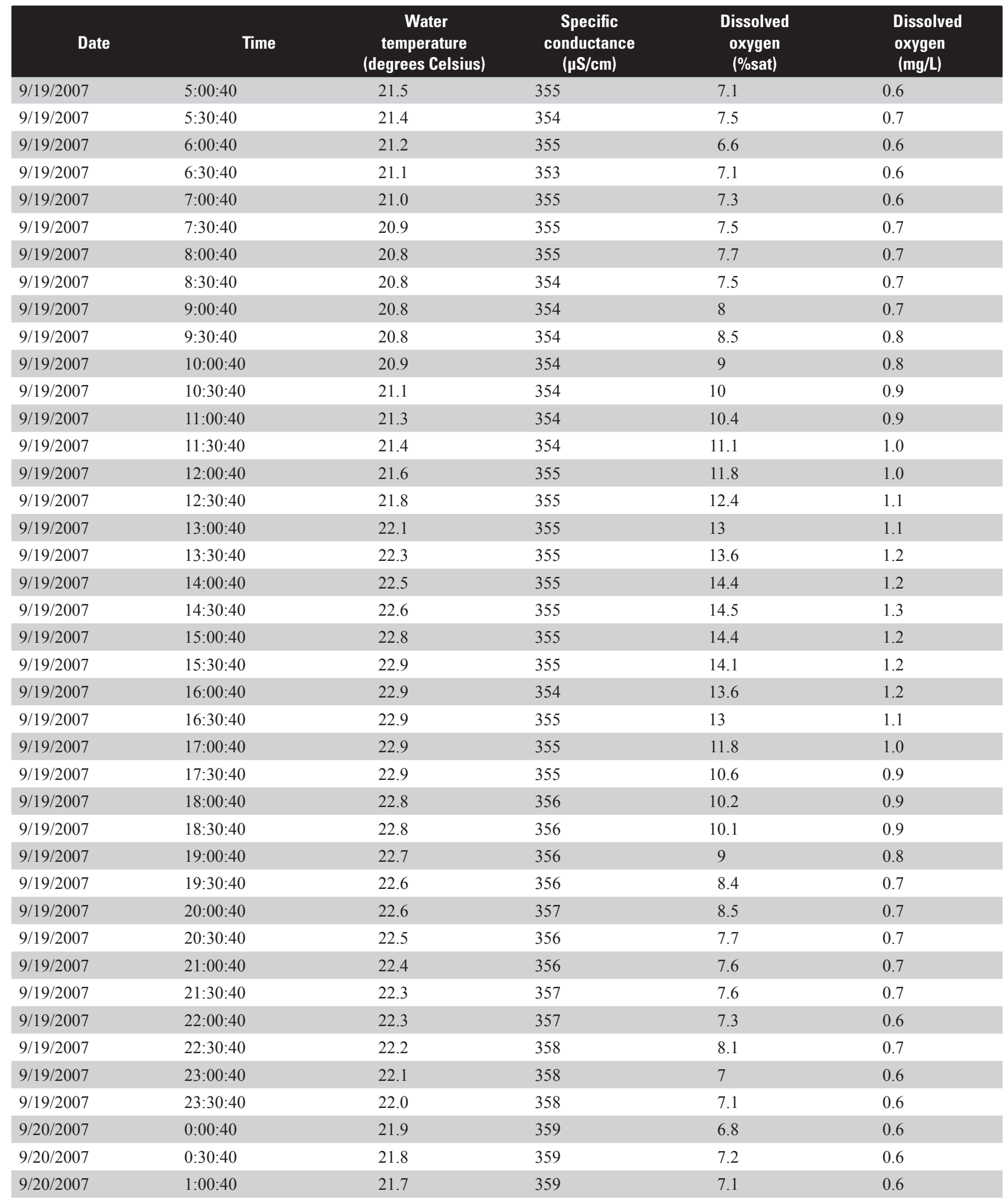


Table 25. Physical property data recorded at 30-minute intervals for station 335749090273300 Home Cypress Bayou near Rome, Mississippi, September 17-20, 2007.-Continued

$[\mu \mathrm{S} / \mathrm{cm}$, microsiemens per centimeter; \%sat, percent saturation; $\mathrm{mg} / \mathrm{L}$, milligrams per liter]

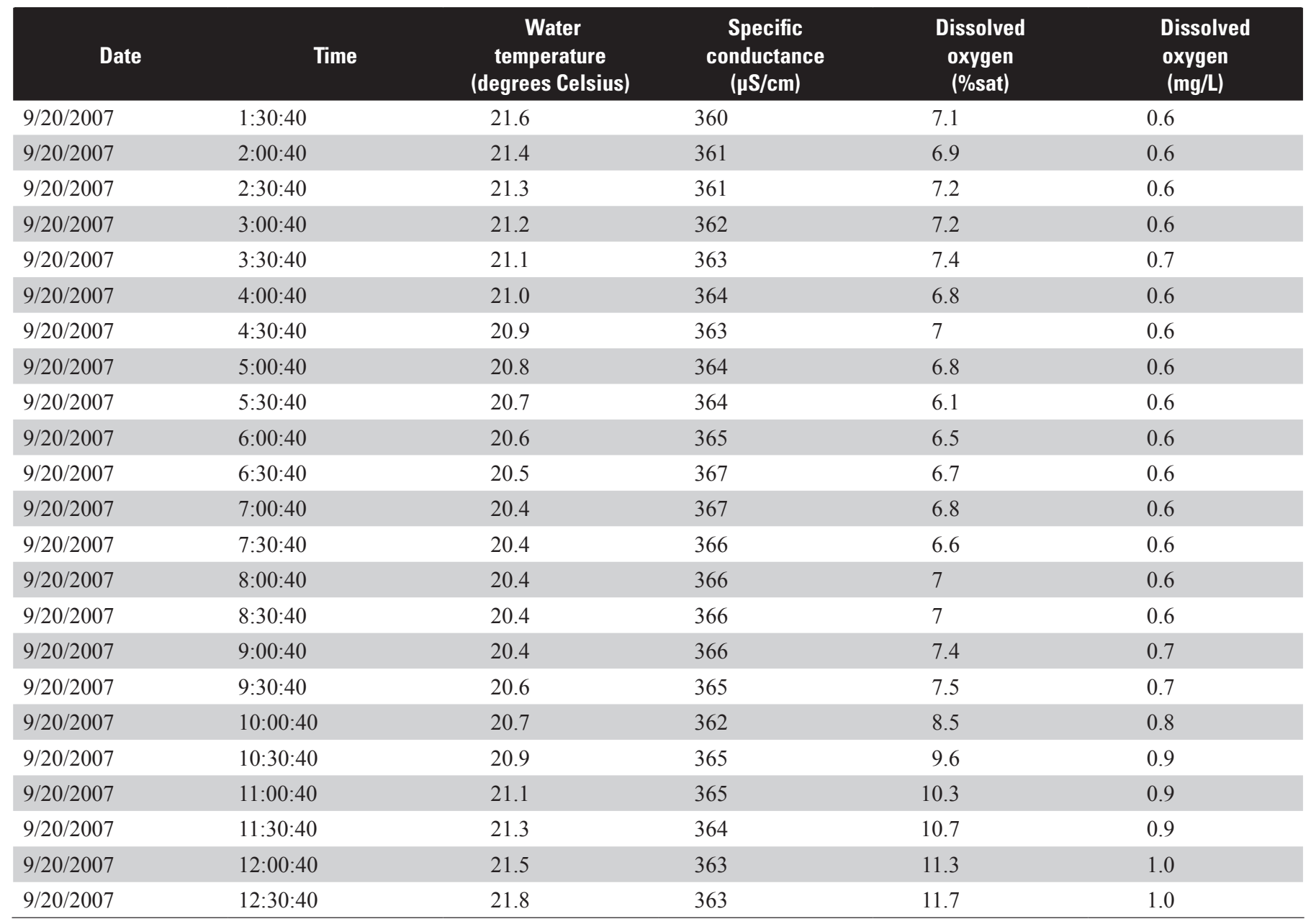


Table 26. Physical property data recorded at 30-minute intervals for station 335919090133600 0possum Bayou near Webb, Mississippi, September 24-27, 2007.

$[\mu \mathrm{S} / \mathrm{cm}$, microsiemens per centimeter; \%sat, percent saturation; $\mathrm{mg} / \mathrm{L}$, milligrams per liter; FNU, formazin nephelometric units; -- , no data]

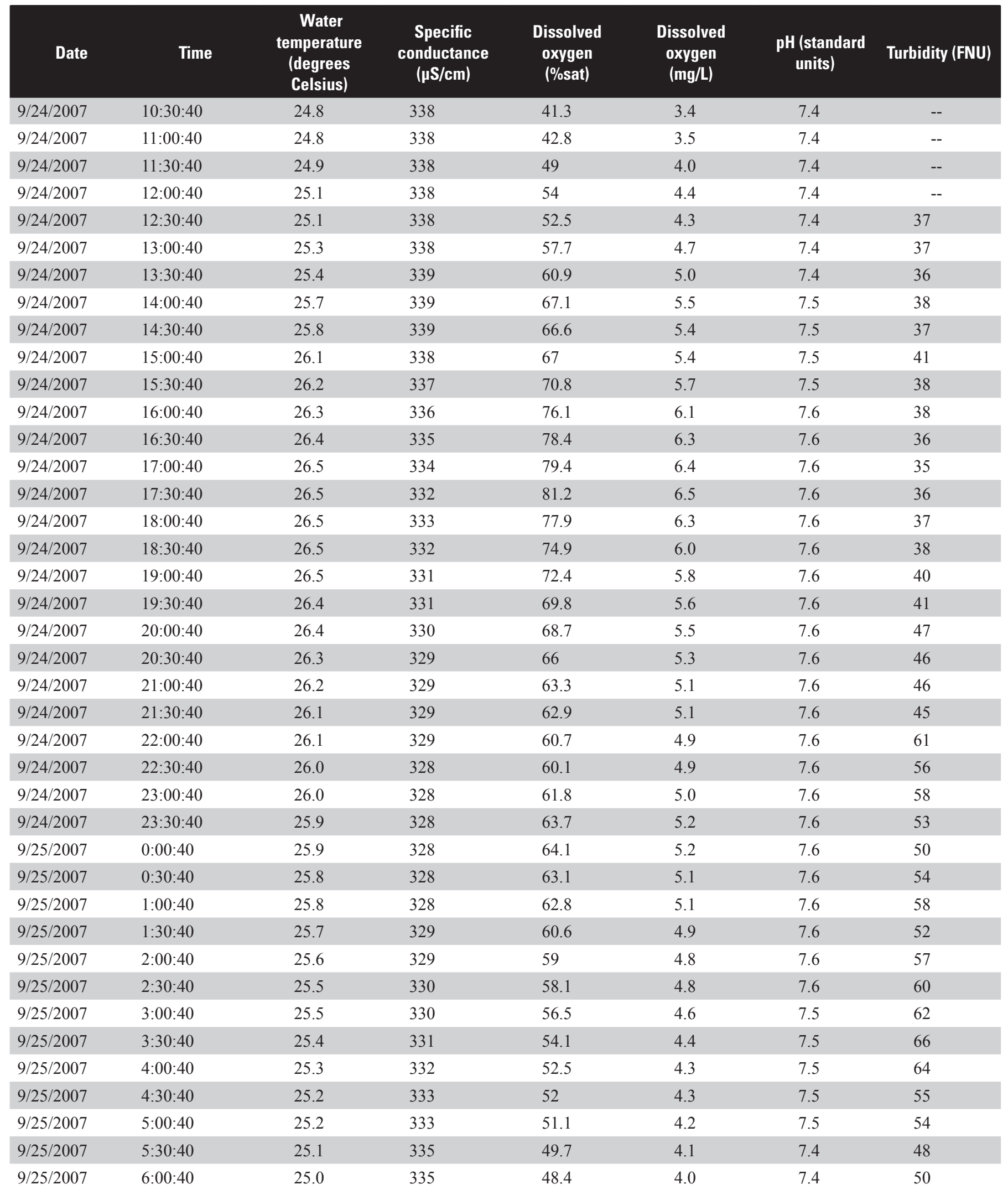


Table 26. Physical property data recorded at 30-minute intervals for station 335919090133600 Opossum Bayou near Webb, Mississippi, September 24-27, 2007.-Continued

$[\mu \mathrm{S} / \mathrm{cm}$, microsiemens per centimeter; \%sat, percent saturation; mg/L, milligrams per liter; FNU, formazin nephelometric units; -- , no data]

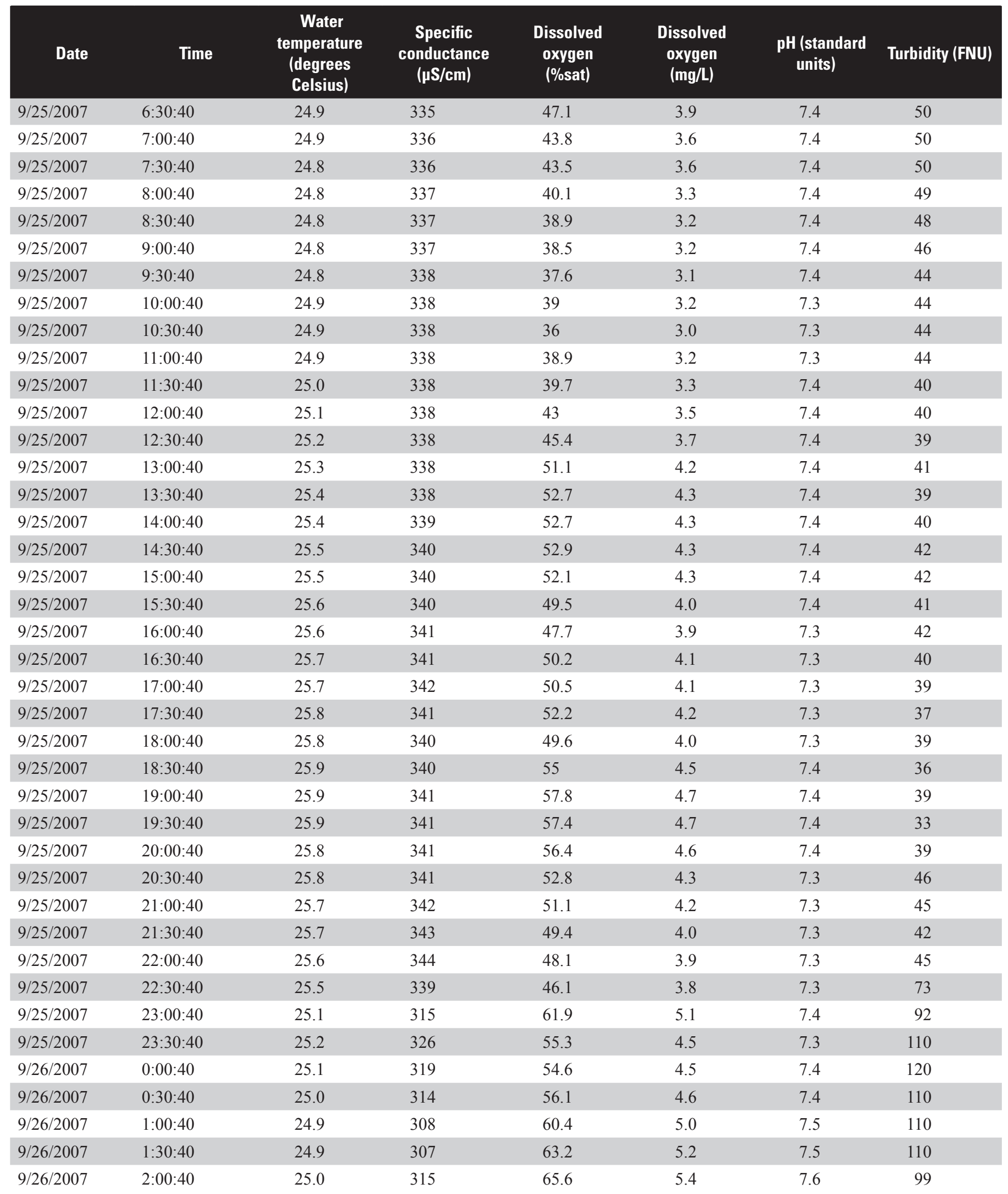


Table 26. Physical property data recorded at 30-minute intervals for station 335919090133600 0possum Bayou near Webb, Mississippi, September 24-27, 2007.-Continued

$[\mu \mathrm{S} / \mathrm{cm}$, microsiemens per centimeter; \%sat, percent saturation; $\mathrm{mg} / \mathrm{L}$, milligrams per liter; FNU, formazin nephelometric units; -- , no data]

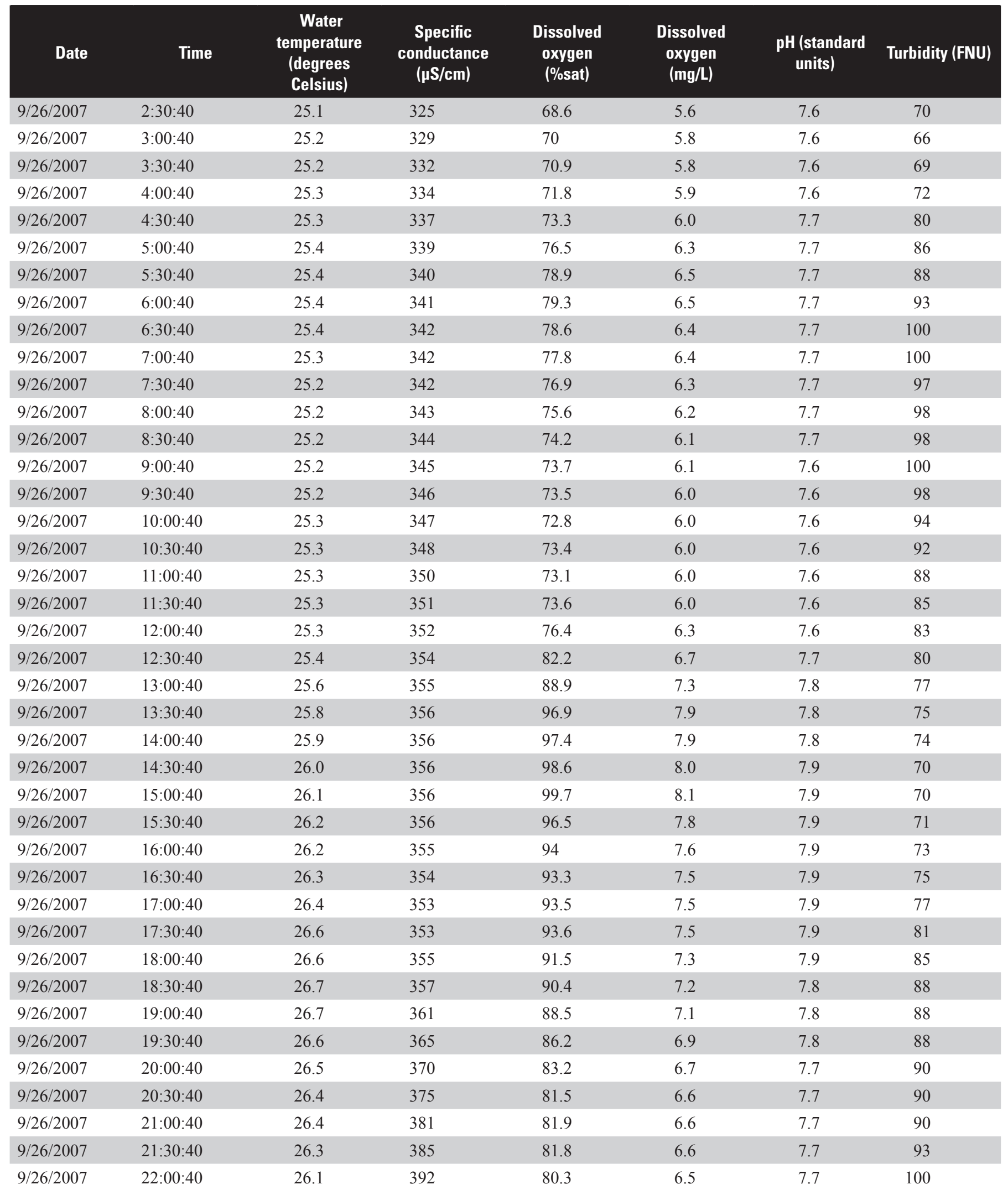


Table 26. Physical property data recorded at 30-minute intervals for station 335919090133600 Opossum Bayou near Webb, Mississippi, September 24-27, 2007.-Continued

$[\mu \mathrm{S} / \mathrm{cm}$, microsiemens per centimeter; \%sat, percent saturation; $\mathrm{mg} / \mathrm{L}$, milligrams per liter; FNU, formazin nephelometric units; -- , no data]

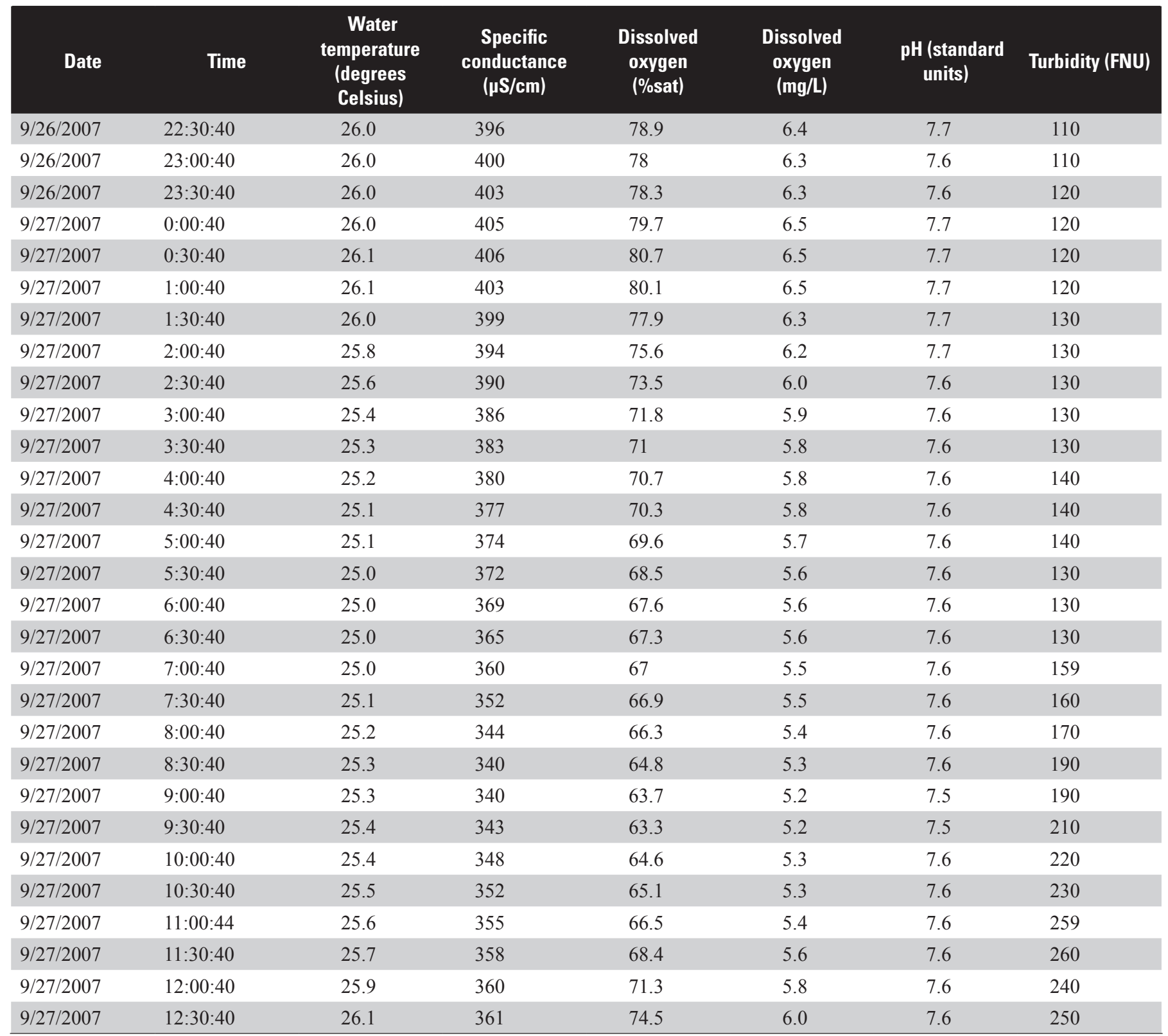


Table 27. Physical property data recorded at 30-minute intervals for station 340157090071200 Unnamed Tributary Panola Floodway near Charleston, Mississippi, September 24-27, 2007.

$[\mu \mathrm{S} / \mathrm{cm}$, microsiemens per centimeter; \%sat, percent saturation; mg/L, milligrams per liter; FNU, formazin nephelometric unit; -- , no data]

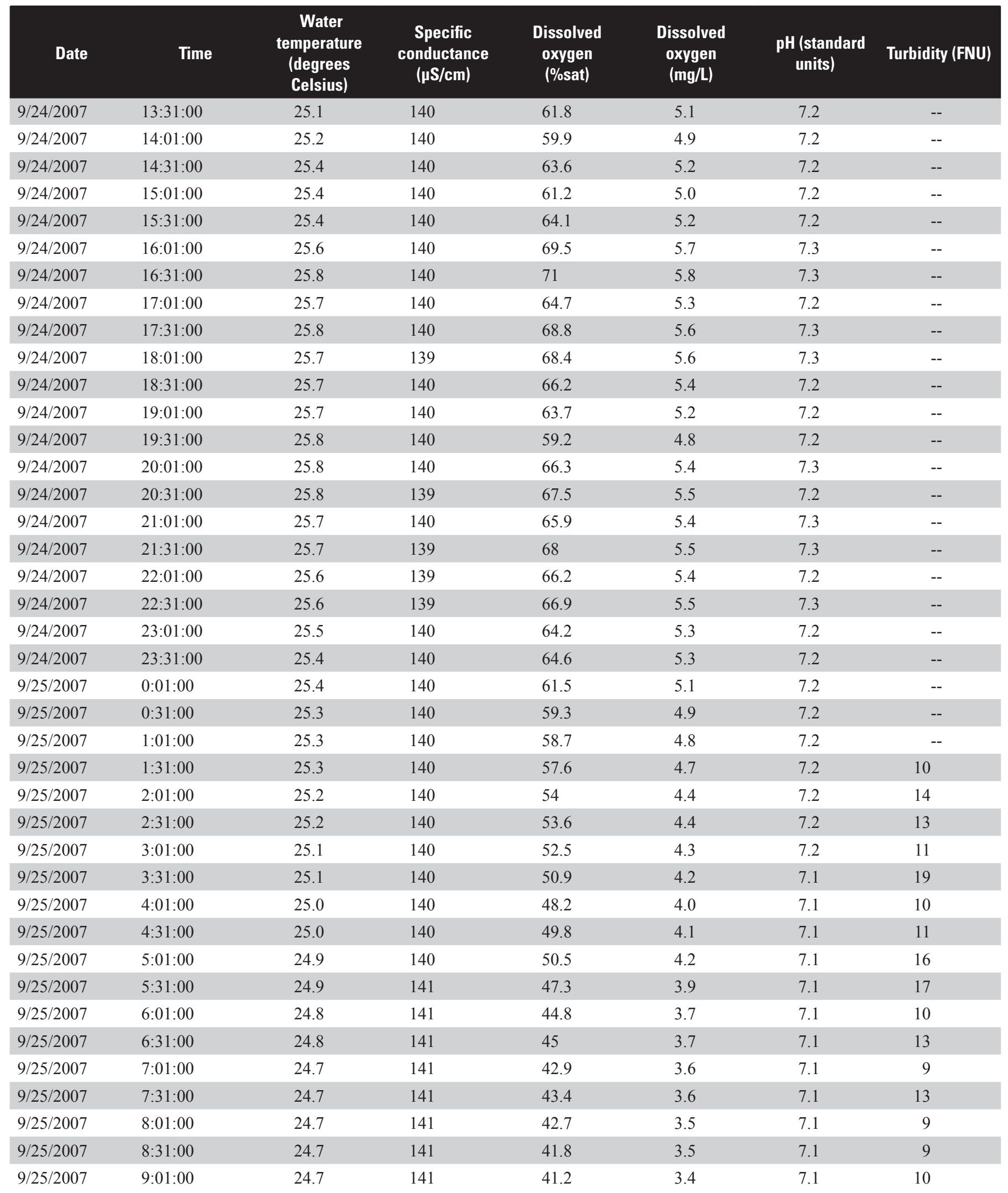


Table 27. Physical property data recorded at 30-minute intervals for station 340157090071200 Unnamed Tributary Panola Floodway near Charleston, Mississippi, September 24-27, 2007.-Continued

$[\mu \mathrm{S} / \mathrm{cm}$, microsiemens per centimeter; \%sat, percent saturation; mg/L, milligrams per liter; FNU, formazin nephelometric unit; -- , no data]

\begin{tabular}{|c|c|c|c|c|c|c|c|}
\hline Date & Time & $\begin{array}{c}\text { Water } \\
\text { temperature } \\
\text { (degrees } \\
\text { Celsius) }\end{array}$ & $\begin{array}{c}\text { Specific } \\
\text { conductance } \\
\text { (pS/cm) }\end{array}$ & $\begin{array}{l}\text { Dissolved } \\
\text { oxygen } \\
\text { (\%sat) }\end{array}$ & $\begin{array}{c}\text { Dissolved } \\
\text { oxygen } \\
\text { (mg/L) }\end{array}$ & $\begin{array}{l}\text { pH (standard } \\
\text { units) }\end{array}$ & Turbidity (FNU) \\
\hline $9 / 25 / 2007$ & 10:01:00 & 24.8 & 141 & 44.1 & 3.6 & 7.1 & 16 \\
\hline $9 / 25 / 2007$ & 10:31:00 & 24.8 & 141 & 42.2 & 3.5 & 7.1 & 14 \\
\hline $9 / 25 / 2007$ & 11:31:00 & 25.0 & 141 & 41.9 & 3.5 & 7.1 & 17 \\
\hline $9 / 25 / 2007$ & 12:01:00 & 25.1 & 141 & 43.1 & 3.5 & 7.1 & 21 \\
\hline $9 / 25 / 2007$ & $12: 31: 00$ & 25.2 & 141 & 43.5 & 3.6 & 7.1 & 17 \\
\hline $9 / 25 / 2007$ & 13:01:00 & 25.4 & 141 & 51.6 & 4.2 & 7.1 & 21 \\
\hline $9 / 25 / 2007$ & 15:01:00 & 25.6 & 137 & 63.7 & 5.2 & 7.2 & 32 \\
\hline $9 / 25 / 2007$ & $15: 31: 00$ & 25.5 & 138 & 57.1 & 4.7 & 7.2 & 25 \\
\hline $9 / 25 / 2007$ & 16:01:00 & 25.7 & 138 & 68.1 & 5.5 & 7.2 & 26 \\
\hline $9 / 25 / 2007$ & $16: 31: 00$ & 25.8 & 138 & 63.5 & 5.2 & 7.2 & 23 \\
\hline $9 / 25 / 2007$ & 17:01:00 & 25.6 & 138 & 63.2 & 5.2 & 7.2 & 31 \\
\hline $9 / 25 / 2007$ & $17: 31: 00$ & 25.7 & 138 & 63.7 & 5.2 & 7.2 & 28 \\
\hline $9 / 25 / 2007$ & 18:01:00 & 25.7 & 138 & 58.7 & 4.8 & 7.2 & 17 \\
\hline $9 / 25 / 2007$ & 18:31:00 & 25.7 & 139 & 59.4 & 4.8 & 7.2 & 15 \\
\hline $9 / 25 / 2007$ & 19:01:00 & 25.6 & 139 & 58.1 & 4.8 & 7.2 & 16 \\
\hline $9 / 25 / 2007$ & $22: 31: 00$ & 25.4 & 140 & 52.2 & 4.3 & 7.2 & 9 \\
\hline $9 / 25 / 2007$ & 23:01:00 & 25.3 & 139 & 54.4 & 4.5 & 7.2 & 13 \\
\hline $9 / 25 / 2007$ & $23: 31: 00$ & 25.3 & 139 & 54.6 & 4.5 & 7.2 & 16 \\
\hline $9 / 26 / 2007$ & 0:01:00 & 25.2 & 139 & 54.3 & 4.5 & 7.2 & 16 \\
\hline $9 / 26 / 2007$ & 0:31:00 & 25.2 & 139 & 54.5 & 4.5 & 7.2 & 16 \\
\hline $9 / 26 / 2007$ & 1:01:00 & 25.1 & 139 & 51.6 & 4.3 & 7.2 & 14 \\
\hline $9 / 26 / 2007$ & 1:31:00 & 25.1 & 139 & 53.2 & 4.4 & 7.2 & 13 \\
\hline $9 / 26 / 2007$ & 2:01:00 & 25.0 & 138 & 52.7 & 4.3 & 7.2 & 13 \\
\hline $9 / 26 / 2007$ & $2: 31: 00$ & 24.9 & 138 & 50.1 & 4.2 & 7.1 & 13 \\
\hline $9 / 26 / 2007$ & 3:01:00 & 24.9 & 138 & 49.7 & 4.1 & 7.1 & 17 \\
\hline $9 / 26 / 2007$ & $3: 31: 00$ & 24.9 & 138 & 50.1 & 4.2 & 7.1 & 19 \\
\hline $9 / 26 / 2007$ & 4:01:00 & 24.8 & 139 & 49.1 & 4.1 & 7.1 & 18 \\
\hline $9 / 26 / 2007$ & $4: 31: 00$ & 24.8 & 139 & 47.1 & 3.9 & 7.1 & 15 \\
\hline $9 / 26 / 2007$ & 5:01:00 & 24.7 & 139 & 47.6 & 4.0 & 7.1 & 17 \\
\hline
\end{tabular}


Table 27. Physical property data recorded at 30-minute intervals for station 340157090071200 Unnamed Tributary Panola Floodway near Charleston, Mississippi, September 24-27, 2007.-Continued

[ $\mu \mathrm{S} / \mathrm{cm}$, microsiemens per centimeter; \%sat, percent saturation; mg/L, milligrams per liter; FNU, formazin nephelometric unit; -- , no data]

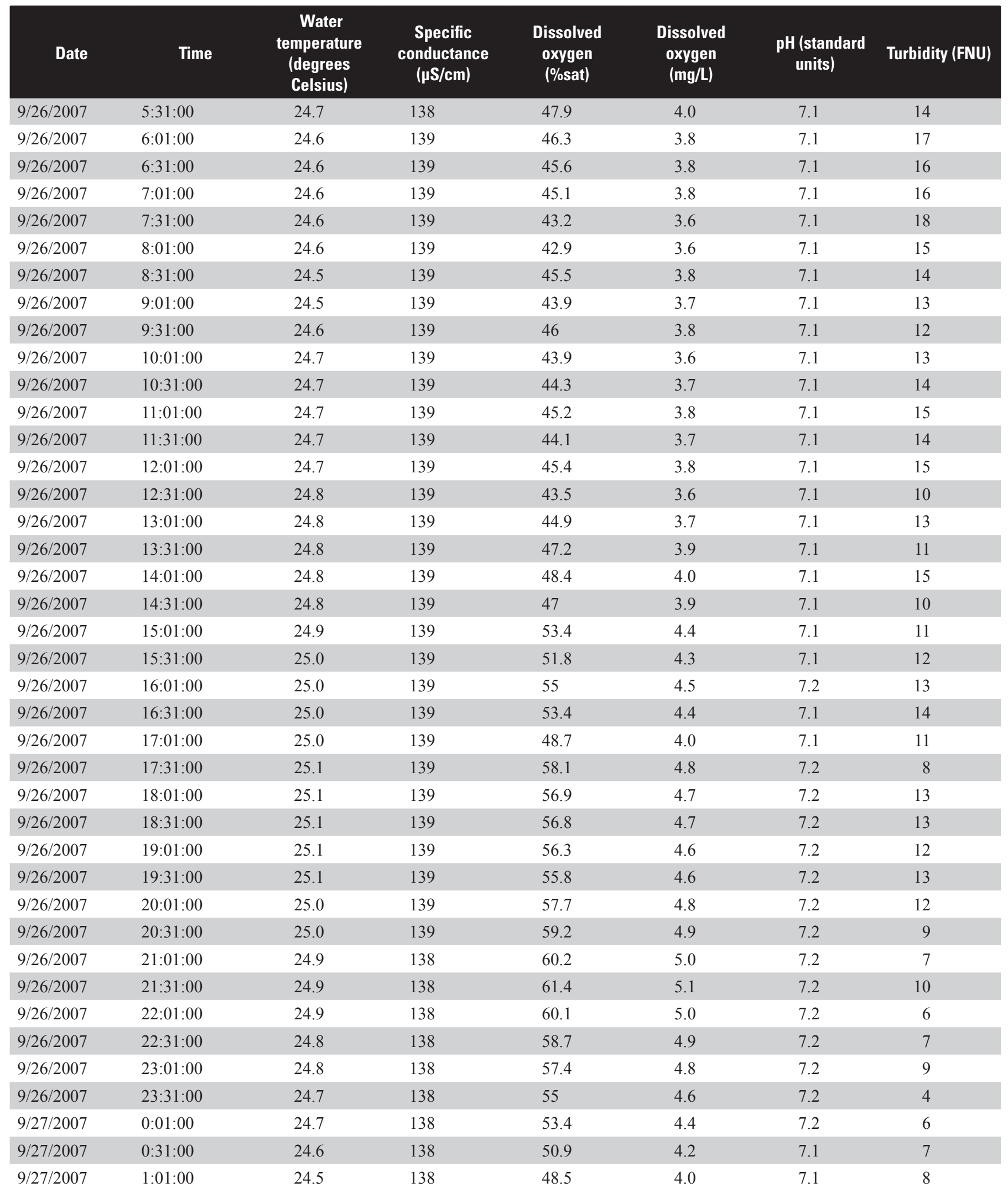


Table 27. Physical property data recorded at 30-minute intervals for station 340157090071200 Unnamed Tributary Panola Floodway near Charleston, Mississippi, September 24-27, 2007.-Continued

$[\mu \mathrm{S} / \mathrm{cm}$, microsiemens per centimeter; \%sat, percent saturation; mg/L, milligrams per liter; FNU, formazin nephelometric unit; -- , no data]

\begin{tabular}{|c|c|c|c|c|c|c|c|}
\hline Date & Time & $\begin{array}{c}\text { Water } \\
\text { temperature } \\
\text { (degrees } \\
\text { Celsius) }\end{array}$ & $\begin{array}{c}\text { Specific } \\
\text { conductance } \\
\text { (pS/cm) }\end{array}$ & $\begin{array}{c}\text { Dissolved } \\
\text { oxygen } \\
\text { (\%sat) }\end{array}$ & $\begin{array}{c}\text { Dissolved } \\
\text { oxygen } \\
\text { (mg/L) }\end{array}$ & $\begin{array}{l}\text { pH (standard } \\
\text { units) }\end{array}$ & Turbidity (FNU) \\
\hline $9 / 27 / 2007$ & 2:01:00 & 24.4 & 137 & 46.3 & 3.9 & 7.1 & 7 \\
\hline $9 / 27 / 2007$ & $2: 31: 00$ & 24.3 & 137 & 45.1 & 3.8 & 7.1 & 6 \\
\hline $9 / 27 / 2007$ & $3: 31: 00$ & 24.1 & 136 & 42.7 & 3.6 & 7.1 & 9 \\
\hline $9 / 27 / 2007$ & 4:01:00 & 24.1 & 136 & 42.4 & 3.6 & 7.1 & 10 \\
\hline $9 / 27 / 2007$ & 4:31:00 & 24.0 & 136 & 41.5 & 3.5 & 7.1 & 7 \\
\hline $9 / 27 / 2007$ & 5:01:00 & 24.0 & 136 & 41.9 & 3.5 & 7.1 & 6 \\
\hline $9 / 27 / 2007$ & 7:01:00 & 23.8 & 136 & 43.2 & 3.6 & 7.1 & 5 \\
\hline $9 / 27 / 2007$ & $7: 31: 00$ & 23.8 & 136 & 43.4 & 3.7 & 7.1 & 7 \\
\hline $9 / 27 / 2007$ & 8:01:00 & 23.8 & 135 & 45.3 & 3.8 & 7.1 & 8 \\
\hline $9 / 27 / 2007$ & $8: 31: 00$ & 23.8 & 135 & 45.1 & 3.8 & 7.1 & 9 \\
\hline $9 / 27 / 2007$ & 9:01:00 & 23.8 & 135 & 45 & 3.8 & 7.1 & 7 \\
\hline $9 / 27 / 2007$ & $9: 31: 00$ & 24.0 & 135 & 47.8 & 4.0 & 7.1 & 7 \\
\hline $9 / 27 / 2007$ & 10:01:00 & 24.1 & 135 & 49.7 & 4.2 & 7.1 & 6 \\
\hline $9 / 27 / 2007$ & $10: 31: 00$ & 24.1 & 135 & 50.2 & 4.2 & 7.1 & 9 \\
\hline $9 / 27 / 2007$ & 11:01:00 & 24.3 & 135 & 51.3 & 4.3 & 7.1 & 8 \\
\hline
\end{tabular}


Table 28. Physical property data recorded at 30-minute intervals for station 340423090210800 Hopson Bayou at Vance, Mississippi, October 4-9, 2007.

$[\mu \mathrm{S} / \mathrm{cm}$, microsiemens per centimeter; \%sat, percent saturation; $\mathrm{mg} / \mathrm{L}$, milligrams per liter; FNU, formazin nephelometric units]

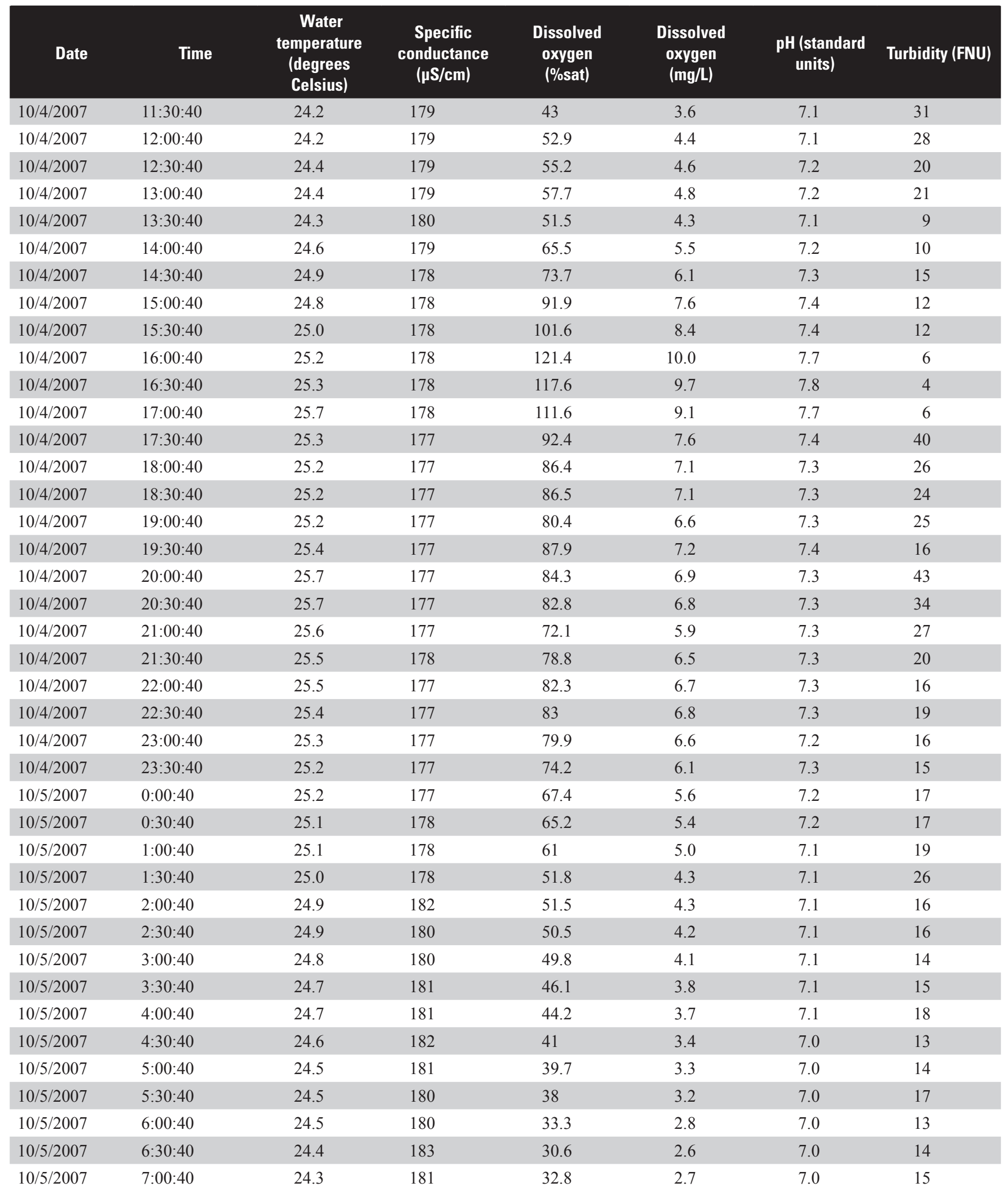


Table 28. Physical property data recorded at 30-minute intervals for station 340423090210800 Hopson Bayou at Vance, Mississippi, October 4-9, 2007.-Continued

$[\mu \mathrm{S} / \mathrm{cm}$, microsiemens per centimeter; \%sat, percent saturation; mg/L, milligrams per liter; FNU, formazin nephelometric units]

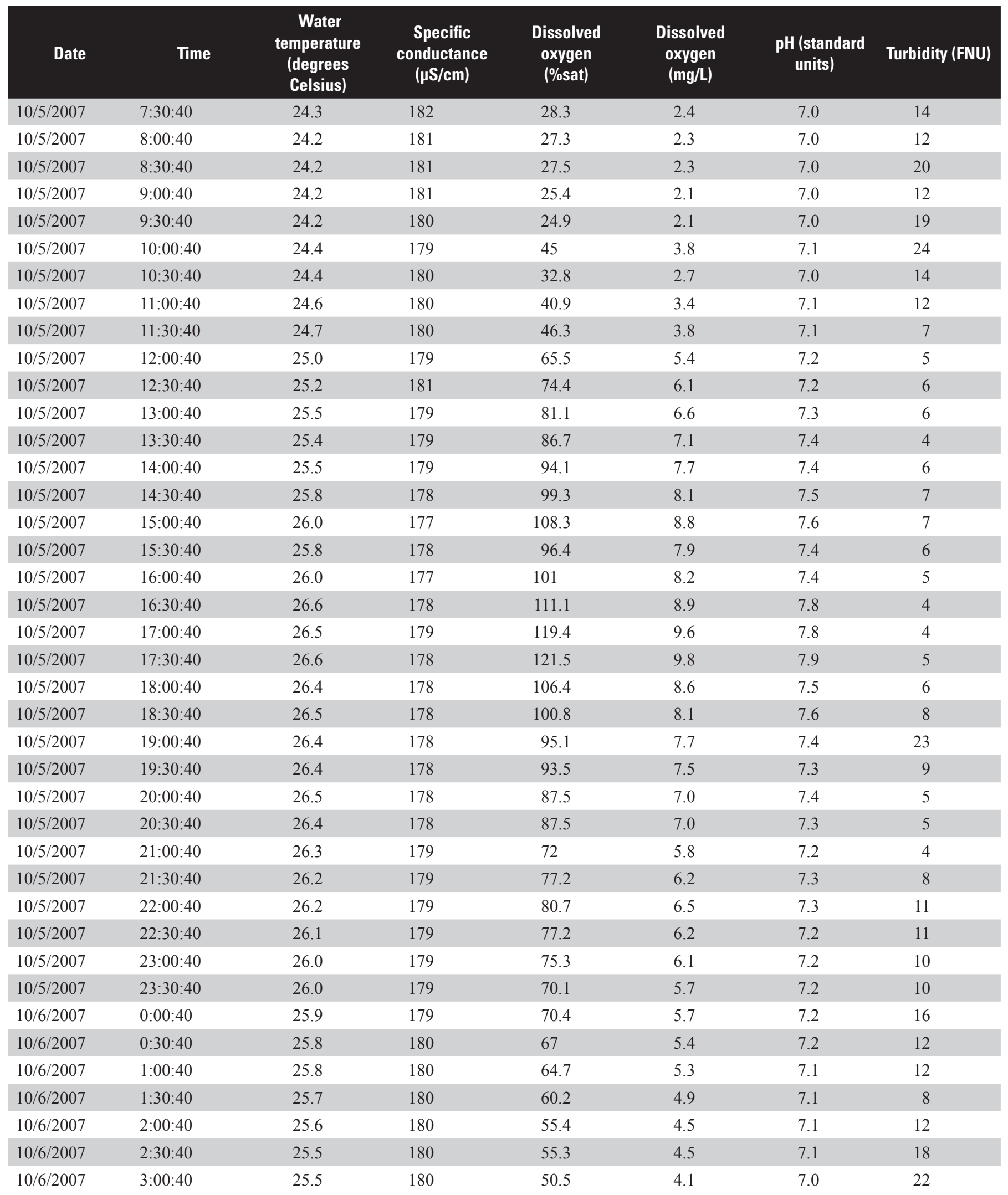


Table 28. Physical property data recorded at 30-minute intervals for station 340423090210800 Hopson Bayou at Vance, Mississippi, October 4-9, 2007.-Continued

$[\mu \mathrm{S} / \mathrm{cm}$, microsiemens per centimeter; \%sat, percent saturation; $\mathrm{mg} / \mathrm{L}$, milligrams per liter; FNU, formazin nephelometric units]

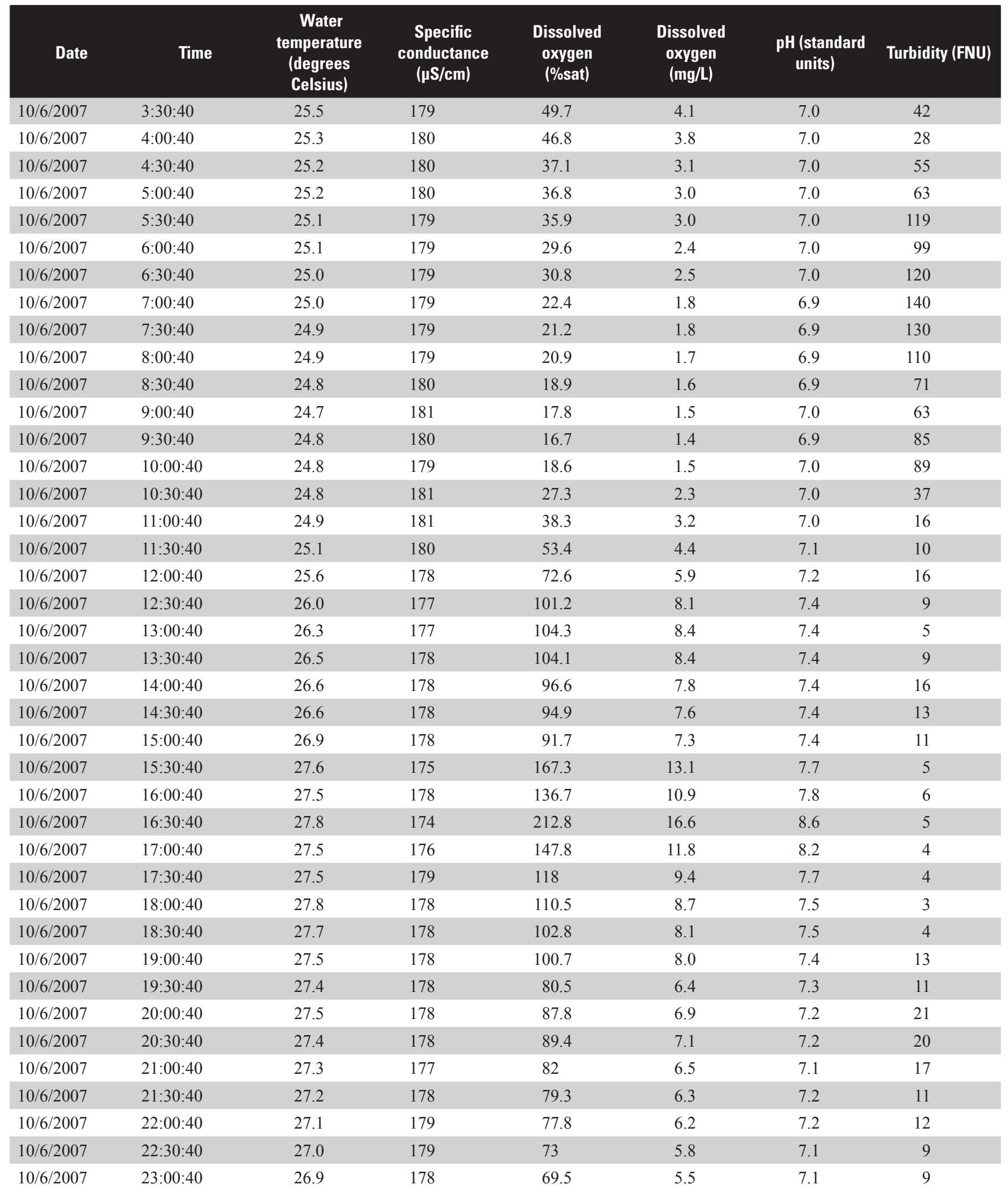


Table 28. Physical property data recorded at 30-minute intervals for station 340423090210800 Hopson Bayou at Vance, Mississippi, October 4-9, 2007.-Continued

$[\mu \mathrm{S} / \mathrm{cm}$, microsiemens per centimeter; \%sat, percent saturation; mg/L, milligrams per liter; FNU, formazin nephelometric units]

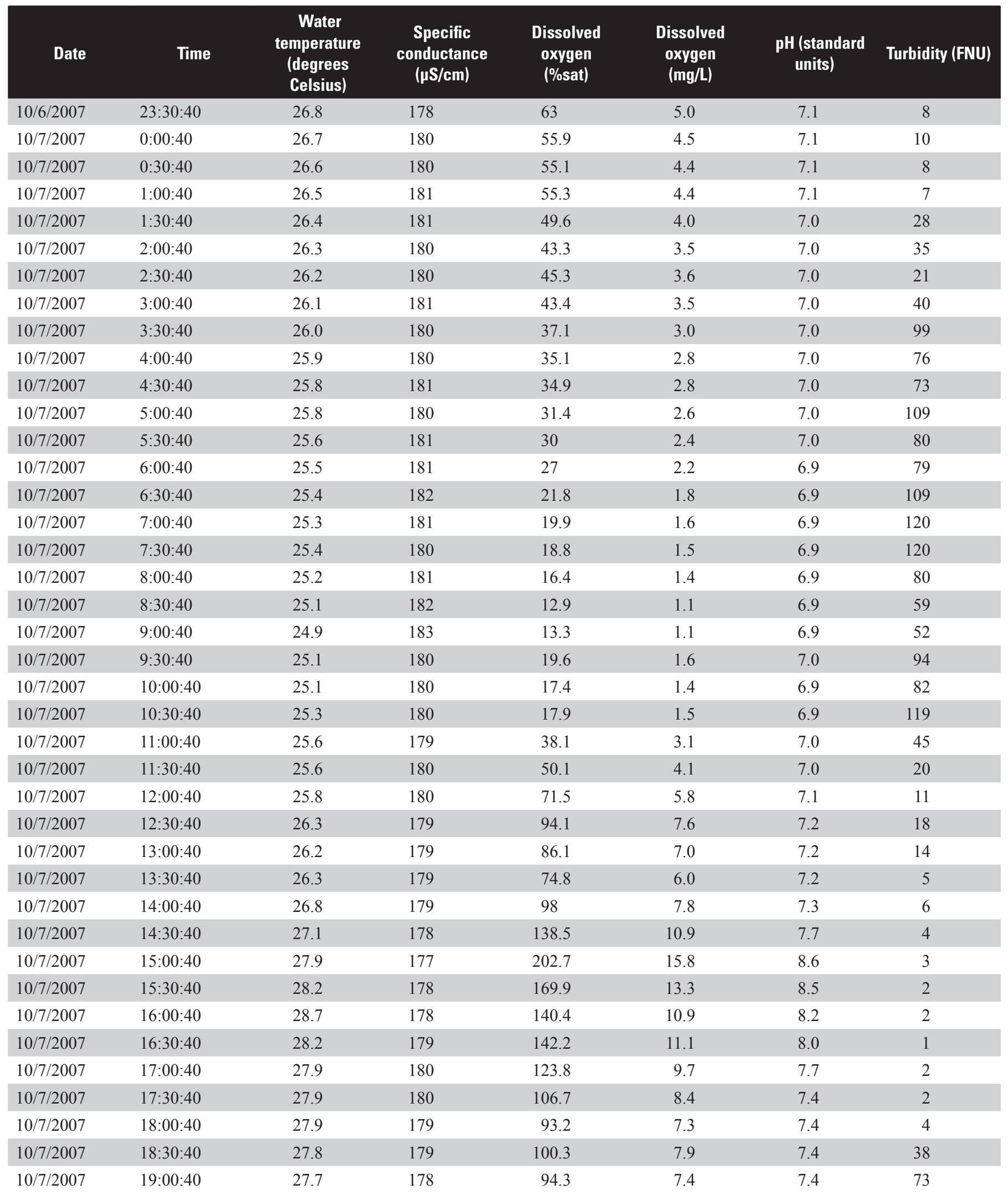


Table 28. Physical property data recorded at 30-minute intervals for station 340423090210800 Hopson Bayou at Vance, Mississippi, October 4-9, 2007.-Continued

$[\mu \mathrm{S} / \mathrm{cm}$, microsiemens per centimeter; \%sat, percent saturation; $\mathrm{mg} / \mathrm{L}$, milligrams per liter; FNU, formazin nephelometric units]

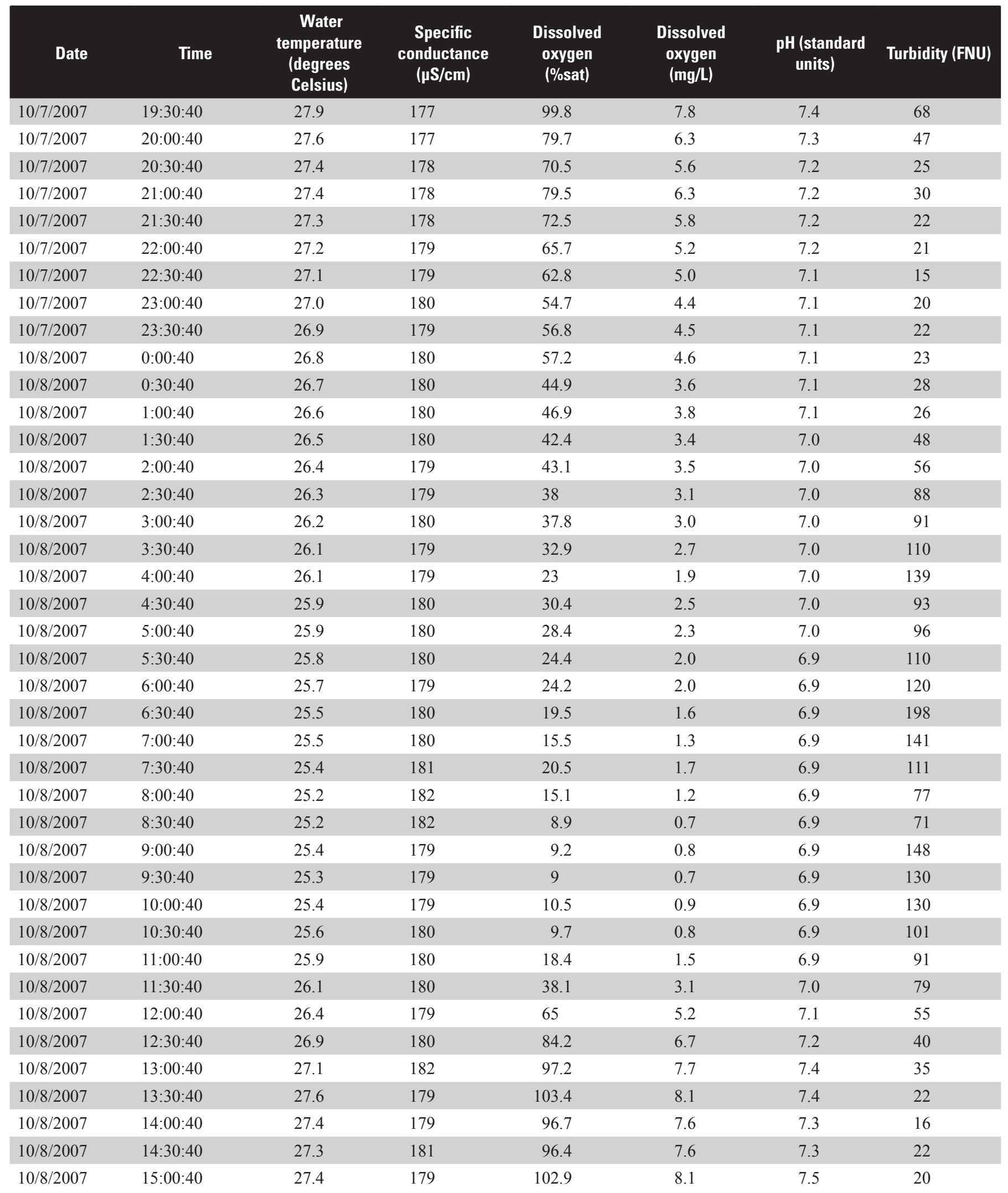


Table 28. Physical property data recorded at 30-minute intervals for station 340423090210800 Hopson Bayou at Vance, Mississippi, October 4-9, 2007.-Continued

$[\mu \mathrm{S} / \mathrm{cm}$, microsiemens per centimeter; \%sat, percent saturation; mg/L, milligrams per liter; FNU, formazin nephelometric units]

\begin{tabular}{|c|c|c|c|c|c|c|c|}
\hline Date & Time & $\begin{array}{c}\text { Water } \\
\text { temperature } \\
\text { (degrees } \\
\text { Celsius) }\end{array}$ & $\begin{array}{c}\text { Specific } \\
\text { conductance } \\
\text { (pS/cm) }\end{array}$ & $\begin{array}{c}\text { Dissolved } \\
\text { oxygen } \\
\text { (\%sat) }\end{array}$ & $\begin{array}{c}\text { Dissolved } \\
\text { oxygen } \\
\text { (mg/L) }\end{array}$ & $\begin{array}{l}\text { pH (standard } \\
\text { units) }\end{array}$ & Turbidity (FNU) \\
\hline $10 / 8 / 2007$ & 16:00:40 & 27.5 & 178 & 107.5 & 8.5 & 7.5 & 33 \\
\hline $10 / 8 / 2007$ & $16: 30: 40$ & 27.5 & 177 & 109.5 & 8.6 & 7.4 & 70 \\
\hline $10 / 8 / 2007$ & $17: 30: 40$ & 28.2 & 179 & 121.3 & 9.4 & 7.6 & 23 \\
\hline $10 / 8 / 2007$ & 18:00:40 & 28.1 & 178 & 109.5 & 8.6 & 7.5 & 19 \\
\hline $10 / 8 / 2007$ & $18: 30: 40$ & 27.8 & 178 & 103.3 & 8.1 & 7.4 & 21 \\
\hline $10 / 8 / 2007$ & 19:00:40 & 27.6 & 178 & 101.5 & 8.0 & 7.4 & 27 \\
\hline $10 / 8 / 2007$ & 21:00:40 & 26.8 & 179 & 76.6 & 6.1 & 7.3 & 40 \\
\hline $10 / 8 / 2007$ & $21: 30: 40$ & 26.7 & 178 & 71.5 & 5.7 & 7.2 & 47 \\
\hline $10 / 8 / 2007$ & $22: 00: 40$ & 26.6 & 178 & 62.2 & 5.0 & 7.2 & 47 \\
\hline $10 / 8 / 2007$ & $22: 30: 40$ & 26.5 & 177 & 64.4 & 5.2 & 7.2 & 49 \\
\hline $10 / 8 / 2007$ & $23: 00: 40$ & 26.4 & 176 & 67.7 & 5.4 & 7.2 & 46 \\
\hline $10 / 8 / 2007$ & $23: 30: 40$ & 26.3 & 176 & 61.8 & 5.0 & 7.2 & 47 \\
\hline $10 / 9 / 2007$ & 0:00:40 & 26.2 & 177 & 56 & 4.5 & 7.1 & 43 \\
\hline
\end{tabular}


Table 29. Physical property data recorded at 30-minute intervals for station 341640090055100 Unnamed Tributary to Tallahatchie River near Locke Station, Mississippi, September 17-19, 2007.

$[\mu \mathrm{S} / \mathrm{cm}$, microsiemens per centimeter; \%sat, percent saturation; mg/L, milligrams per liter; FNU, formazin nephelometric units; -- , no data; E, estimated]

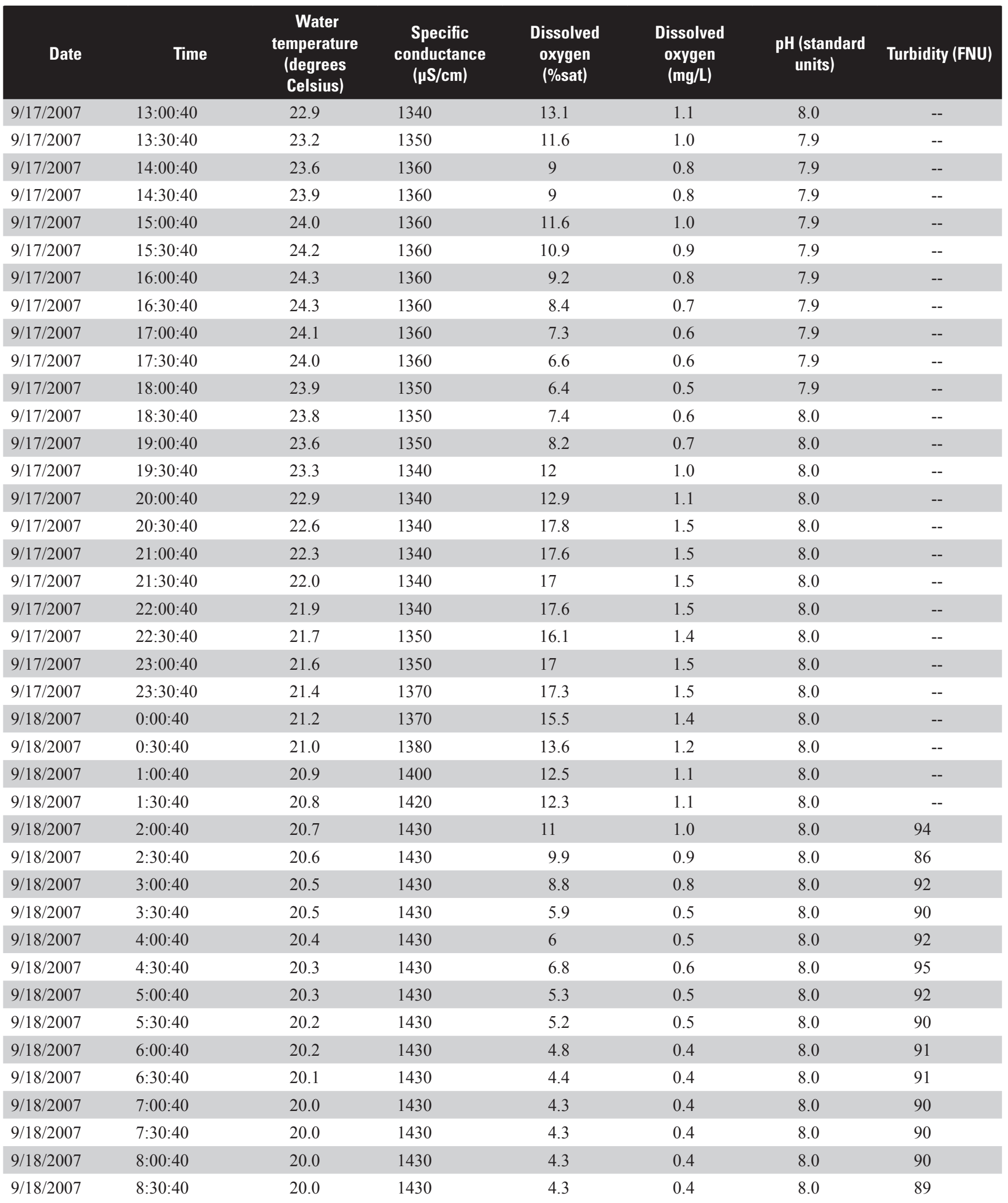


Table 29. Physical property data recorded at 30-minute intervals for station 341640090055100 Unnamed Tributary to Tallahatchie River near Locke Station, Mississippi, September 17-19, 2007.-Continued

$[\mu \mathrm{S} / \mathrm{cm}$, microsiemens per centimeter; \%sat, percent saturation; mg/L, milligrams per liter; FNU, formazin nephelometric units; -- , no data; E, estimated]

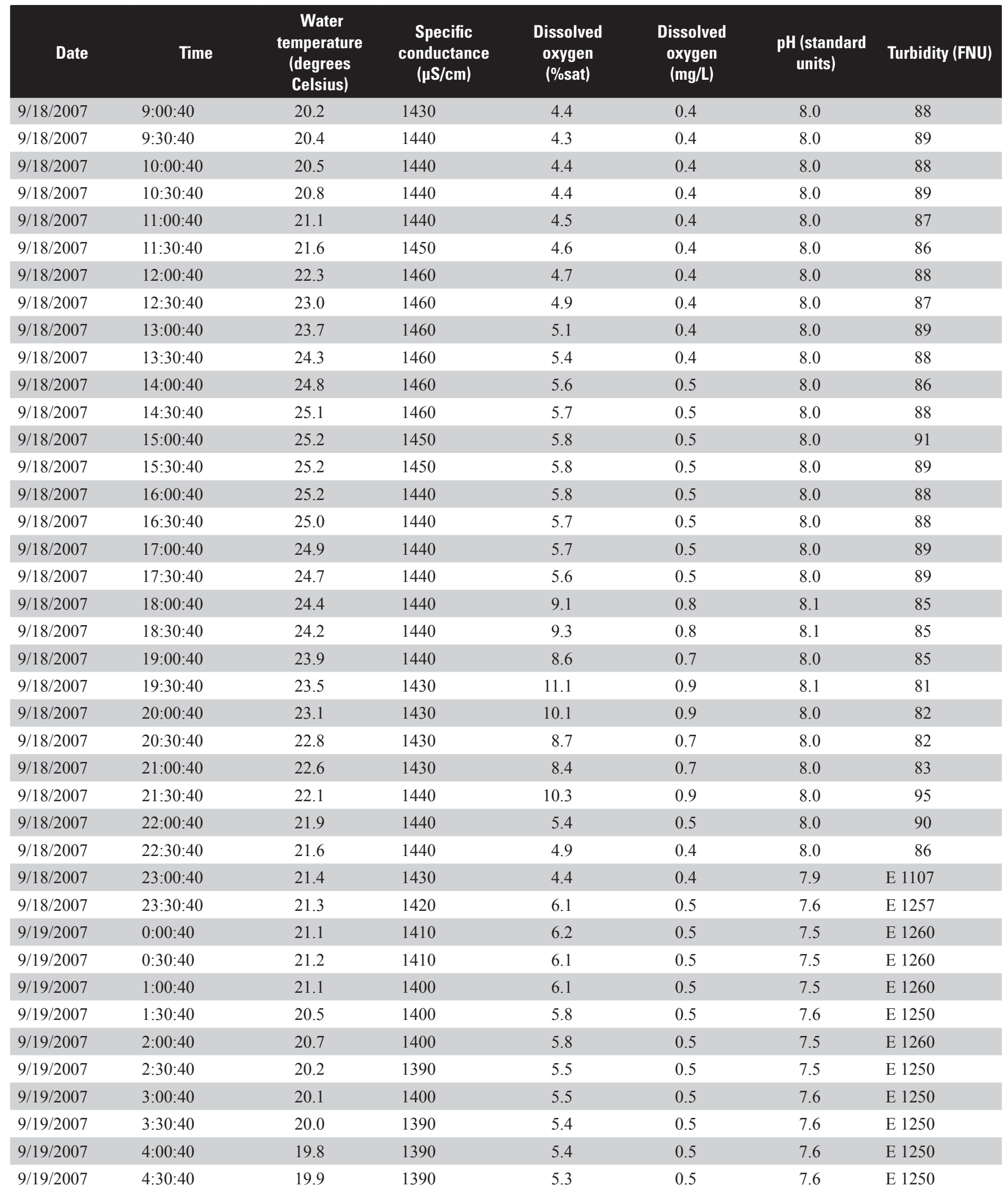


Table 29. Physical property data recorded at 30-minute intervals for station 341640090055100 Unnamed Tributary to Tallahatchie River near Locke Station, Mississippi, September 17-19, 2007.-Continued

$[\mu \mathrm{S} / \mathrm{cm}$, microsiemens per centimeter; \%sat, percent saturation; mg/L, milligrams per liter; FNU, formazin nephelometric units; -- , no data; E, estimated]

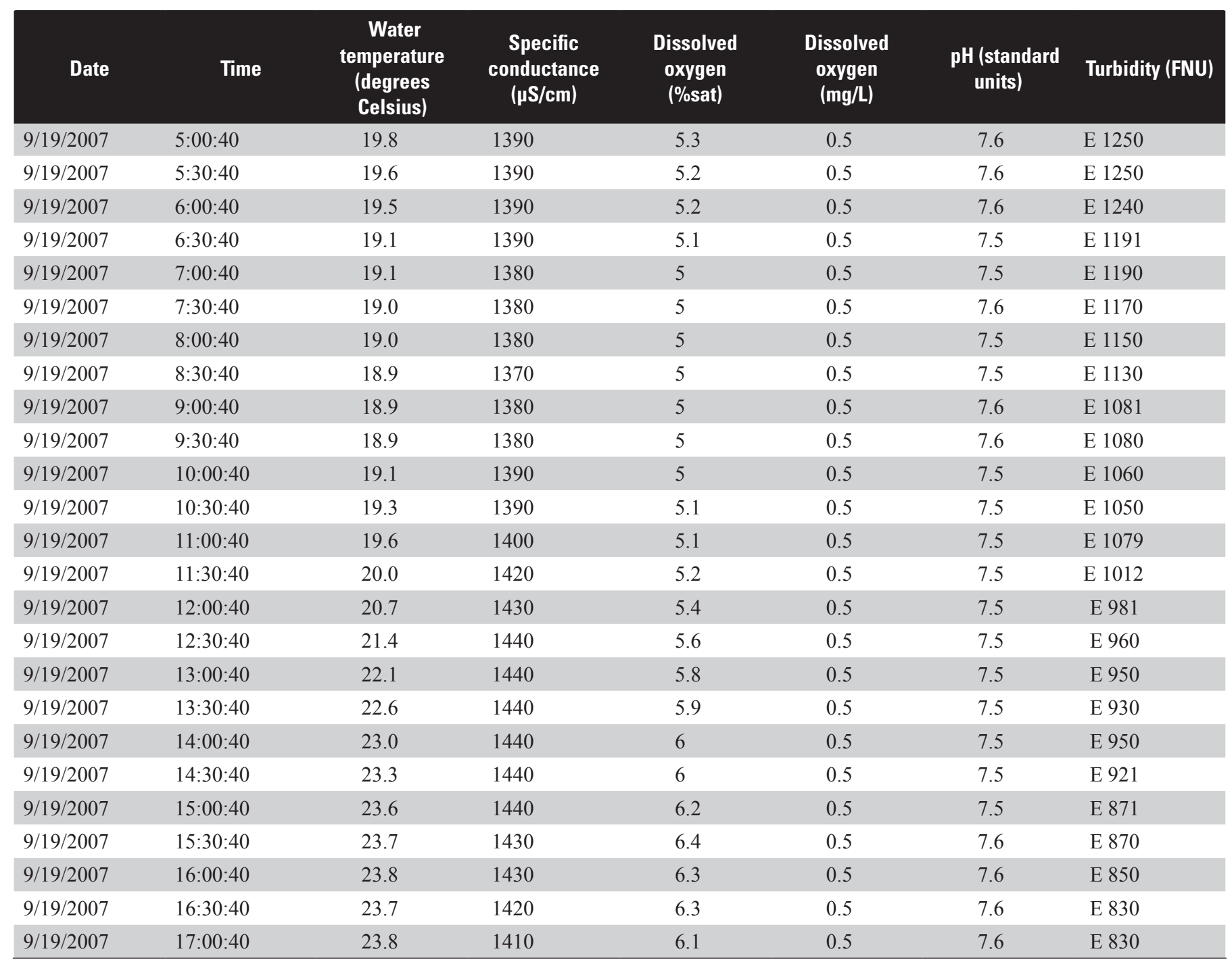


Table 30. Physical property data recorded at 30-minute intervals for station 341701090113300 Ash Log Bayou near Bobo, Mississippi, September 17-19, 2007.

$[\mu \mathrm{S} / \mathrm{cm}$, microsiemens per centimeter; \%sat, percent saturation; mg/L, milligrams per liter; FNU, formazin nephelometric units; -- , no data]

\begin{tabular}{|c|c|c|c|c|c|c|c|}
\hline Date & Time & $\begin{array}{c}\text { Water } \\
\text { temperature } \\
\text { (degrees } \\
\text { Celsius) }\end{array}$ & $\begin{array}{c}\text { Specific } \\
\text { conductance } \\
\text { (pS/cm) }\end{array}$ & $\begin{array}{c}\text { Dissolved } \\
\text { oxygen } \\
\text { (\%sat) }\end{array}$ & $\begin{array}{l}\text { Dissolved } \\
\text { oxygen } \\
\text { (mg/L) }\end{array}$ & $\begin{array}{l}\text { pH (standard } \\
\text { units) }\end{array}$ & Turbidity (FNU) \\
\hline 9/17/2007 & 15:01:00 & 25.2 & 359 & 118.4 & 9.7 & 8.2 & 18 \\
\hline $9 / 17 / 2007$ & $15: 31: 00$ & 25.5 & 361 & 120.5 & 9.8 & 8.2 & 26 \\
\hline $9 / 17 / 2007$ & $16: 31: 00$ & 25.9 & 369 & 147.2 & 11.8 & 8.6 & 9 \\
\hline $9 / 17 / 2007$ & 17:01:00 & 26.3 & 360 & 137.2 & 11.1 & 8.5 & 8 \\
\hline $9 / 17 / 2007$ & $17: 31: 00$ & 26.3 & 356 & 124.5 & 10.0 & 8.3 & 17 \\
\hline $9 / 17 / 2007$ & 18:01:00 & 26.1 & 366 & 136.3 & 11.0 & 8.5 & 13 \\
\hline $9 / 17 / 2007$ & 20:01:00 & 25.6 & 365 & 121.3 & 9.9 & 8.4 & 24 \\
\hline $9 / 17 / 2007$ & $20: 31: 00$ & 25.4 & 368 & 122.7 & 10.1 & 8.5 & 26 \\
\hline $9 / 17 / 2007$ & 21:01:00 & 25.3 & 369 & 120.5 & 9.9 & 8.4 & 28 \\
\hline $9 / 17 / 2007$ & $21: 31: 00$ & 25.1 & 373 & 123 & 10.1 & 8.6 & 18 \\
\hline $9 / 17 / 2007$ & $22: 01: 00$ & 25.0 & 375 & 121.8 & 10.1 & 8.6 & 22 \\
\hline $9 / 17 / 2007$ & $22: 31: 00$ & 24.8 & 377 & 119.3 & 9.9 & 8.5 & 26 \\
\hline $9 / 17 / 2007$ & 23:01:00 & 24.7 & 378 & 118.6 & 9.8 & 8.6 & 27 \\
\hline $9 / 17 / 2007$ & $23: 31: 00$ & 24.6 & 378 & 120.7 & 10.0 & 8.6 & 19 \\
\hline 9/18/2007 & 0:01:00 & 24.4 & 378 & 121.2 & 10.1 & 8.6 & 17 \\
\hline $9 / 18 / 2007$ & $3: 31: 00$ & 23.6 & 379 & 121.1 & 10.2 & 8.7 & 10 \\
\hline 9/18/2007 & 4:01:00 & 23.6 & 380 & 120.1 & 10.2 & 8.6 & 10 \\
\hline $9 / 18 / 2007$ & $4: 31: 00$ & 23.5 & 380 & 117 & 9.9 & 8.6 & 18 \\
\hline $9 / 18 / 2007$ & 5:01:00 & 23.4 & 380 & 114.9 & 9.8 & 8.6 & 28 \\
\hline $9 / 18 / 2007$ & $5: 31: 00$ & 23.3 & 380 & 112.9 & 9.6 & 8.6 & 33 \\
\hline $9 / 18 / 2007$ & $6: 01: 00$ & 23.2 & 380 & 110.5 & 9.4 & 8.6 & 32 \\
\hline 9/18/2007 & $6: 31: 00$ & 23.1 & 379 & 107 & 9.2 & 8.5 & 28 \\
\hline 9/18/2007 & 7:01:00 & 23.0 & 380 & 104 & 8.9 & 8.5 & 26 \\
\hline $9 / 18 / 2007$ & $7: 31: 00$ & 22.9 & 381 & 103.9 & 8.9 & 8.5 & 20 \\
\hline 9/18/2007 & 8:01:00 & 22.8 & 380 & 101.1 & 8.7 & 8.4 & 23 \\
\hline $9 / 18 / 2007$ & $8: 31: 00$ & 22.8 & 381 & 102.5 & 8.8 & 8.5 & 19 \\
\hline $9 / 18 / 2007$ & 9:01:00 & 22.7 & 382 & 104.4 & 9.0 & 8.5 & 20 \\
\hline $9 / 18 / 2007$ & $9: 31: 00$ & 23.0 & 381 & 106.1 & 9.1 & 8.5 & 18 \\
\hline 9/18/2007 & 10:01:00 & 22.9 & 380 & 110.6 & 9.5 & 8.6 & 18 \\
\hline
\end{tabular}


Table 30. Physical property data recorded at 30-minute intervals for station 341701090113300 Ash Log Bayou near Bobo, Mississippi, September 17-19, 2007.-Continued

$[\mu \mathrm{S} / \mathrm{cm}$, microsiemens per centimeter; \%sat, percent saturation; mg/L, milligrams per liter; FNU, formazin nephelometric units; -- , no data]

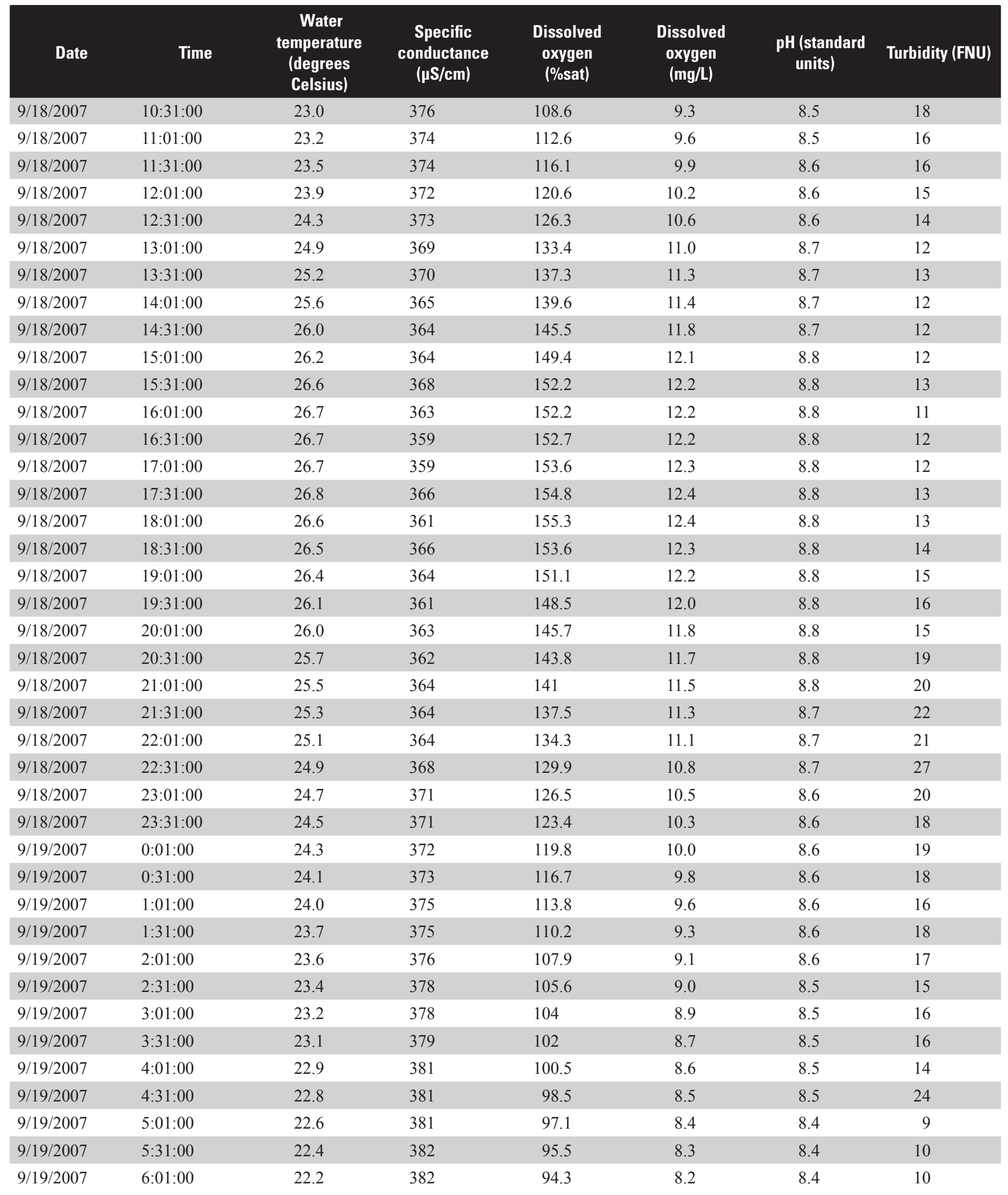


Table 30. Physical property data recorded at 30-minute intervals for station 341701090113300 Ash Log Bayou near Bobo, Mississippi, September 17-19, 2007.-Continued

$[\mu \mathrm{S} / \mathrm{cm}$, microsiemens per centimeter; \%sat, percent saturation; mg/L, milligrams per liter; FNU, formazin nephelometric units; -- , no data]

\begin{tabular}{|c|c|c|c|c|c|c|c|}
\hline Date & Time & $\begin{array}{c}\text { Water } \\
\text { temperature } \\
\text { (degrees } \\
\text { Celsius) }\end{array}$ & $\begin{array}{c}\text { Specific } \\
\text { conductance } \\
\text { (pS/cm) }\end{array}$ & $\begin{array}{c}\text { Dissolved } \\
\text { oxygen } \\
\text { (\%sat) }\end{array}$ & $\begin{array}{l}\text { Dissolved } \\
\text { oxygen } \\
\text { (mg/L) }\end{array}$ & $\begin{array}{l}\text { pH (standard } \\
\text { units) }\end{array}$ & Turbidity (FNU) \\
\hline 9/19/2007 & 7:01:00 & 21.9 & 383 & 90.6 & 7.9 & 8.4 & 12 \\
\hline $9 / 19 / 2007$ & $7: 31: 00$ & 21.8 & 383 & 89 & 7.8 & 8.4 & 13 \\
\hline $9 / 19 / 2007$ & $8: 31: 00$ & 21.8 & 386 & 88.2 & 7.7 & 8.3 & 12 \\
\hline $9 / 19 / 2007$ & 9:01:00 & 21.9 & 386 & 90.5 & 7.9 & 8.4 & 11 \\
\hline $9 / 19 / 2007$ & $9: 31: 00$ & 21.9 & 386 & 90.6 & 7.9 & 8.4 & 11 \\
\hline $9 / 19 / 2007$ & 10:01:00 & 22.2 & 384 & 95.1 & 8.3 & 8.4 & 11 \\
\hline $9 / 19 / 2007$ & 12:01:00 & 23.4 & 378 & 120.3 & 10.2 & 8.6 & 9 \\
\hline $9 / 19 / 2007$ & $12: 31: 00$ & 23.6 & 379 & 119.6 & 10.1 & 8.6 & 10 \\
\hline 9/19/2007 & 13:01:00 & 24.1 & 381 & 123.6 & 10.4 & 8.6 & 9 \\
\hline $9 / 19 / 2007$ & $13: 31: 00$ & 24.6 & 382 & 128.4 & 10.7 & 8.6 & 7 \\
\hline 9/19/2007 & 14:01:00 & 25.1 & 382 & 131.3 & 10.8 & 8.6 & 10 \\
\hline $9 / 19 / 2007$ & $14: 31: 00$ & 25.5 & 377 & 137.8 & 11.3 & 8.7 & 8 \\
\hline $9 / 19 / 2007$ & 15:01:00 & 25.7 & 375 & 146.6 & 11.9 & 8.8 & 6 \\
\hline $9 / 19 / 2007$ & $15: 31: 00$ & 25.9 & 379 & 146.2 & 11.9 & 8.8 & 6 \\
\hline $9 / 19 / 2007$ & 16:01:00 & 26.3 & 378 & 148.1 & 11.9 & 8.7 & 6 \\
\hline
\end{tabular}


Table 31. Physical property data recorded at 30-minute intervals for station 344516090164400 Floyd Bayou, near Green River, Mississippi, September 17-19, 2007.

$[\mu \mathrm{S} / \mathrm{cm}$, microsiemens per centimeter; \%sat, percent saturation; $\mathrm{mg} / \mathrm{L}$, milligrams per liter]

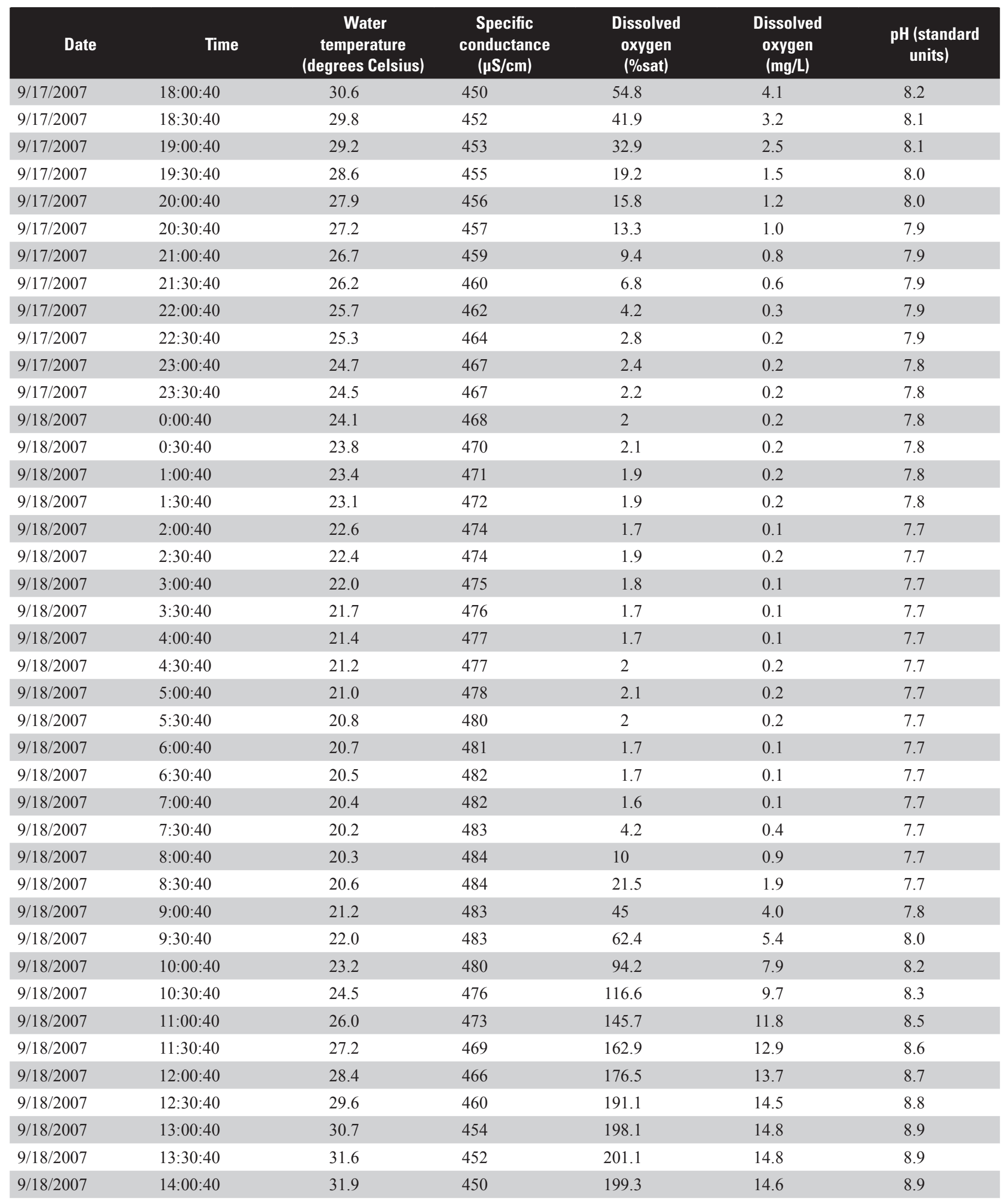


Table 31. Physical property data recorded at 30-minute intervals for station 344516090164400 Floyd Bayou, near Green River, Mississippi, September 17-19, 2007.-Continued

$[\mu \mathrm{S} / \mathrm{cm}$, microsiemens per centimeter; \%sat, percent saturation; $\mathrm{mg} / \mathrm{L}$, milligrams per liter]

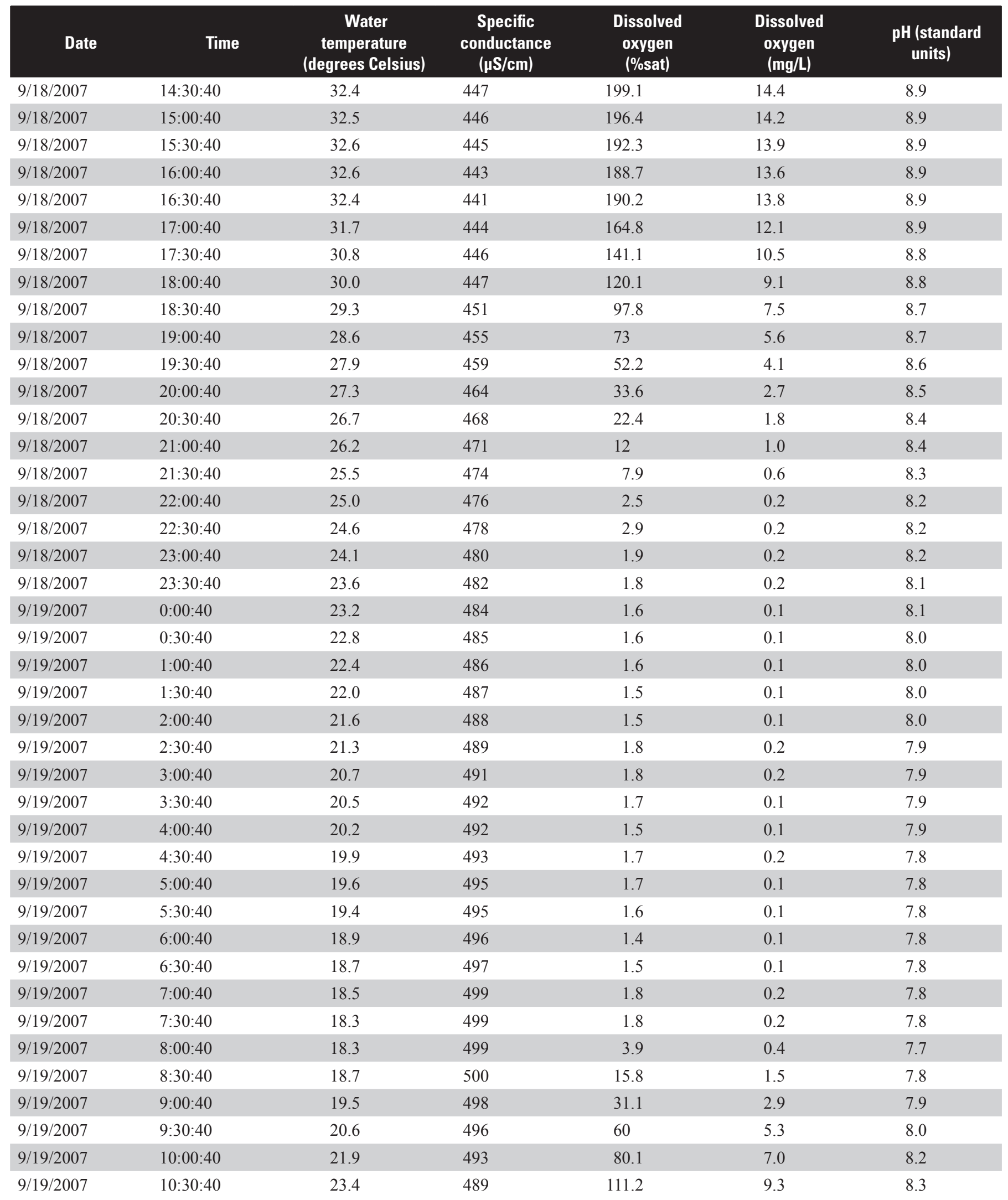


Table 31. Physical property data recorded at 30-minute intervals for station 344516090164400 Floyd Bayou, near Green River, Mississippi, September 17-19, 2007.-Continued

$[\mu \mathrm{S} / \mathrm{cm}$, microsiemens per centimeter; \%sat, percent saturation; $\mathrm{mg} / \mathrm{L}$, milligrams per liter]

\begin{tabular}{|c|c|c|c|c|c|c|}
\hline Date & Time & $\begin{array}{c}\text { Water } \\
\text { temperature } \\
\text { (degrees Celsius) }\end{array}$ & $\begin{array}{c}\text { Specific } \\
\text { conductance } \\
\text { (pS/cm) }\end{array}$ & $\begin{array}{c}\text { Dissolved } \\
\text { oxygen } \\
\text { (\%sat) }\end{array}$ & $\begin{array}{l}\text { Dissolved } \\
\text { oxygen } \\
\text { (mg/L) }\end{array}$ & $\begin{array}{l}\text { pH (standard } \\
\text { units) }\end{array}$ \\
\hline $9 / 19 / 2007$ & $11: 00: 40$ & 24.9 & 485 & 135 & 11.2 & 8.5 \\
\hline $9 / 19 / 2007$ & $11: 30: 40$ & 26.3 & 480 & 163 & 13.1 & 8.6 \\
\hline $9 / 19 / 2007$ & $12: 00: 40$ & 27.7 & 473 & 183.5 & 14.4 & 8.7 \\
\hline $9 / 19 / 2007$ & $13: 30: 40$ & 31.2 & 455 & 208.9 & 15.4 & 8.9 \\
\hline $9 / 19 / 2007$ & $14: 00: 40$ & 31.9 & 450 & 218.6 & 16.0 & 9.0 \\
\hline $9 / 19 / 2007$ & $14: 30: 40$ & 32.7 & 444 & 220.9 & 15.9 & 9.0 \\
\hline $9 / 19 / 2007$ & $15: 00: 40$ & 33.0 & 440 & 220.7 & 15.8 & 9.0 \\
\hline
\end{tabular}



